SOUND USE, SEQUENTIAL BEHAVIOR AND ECOLOGY OF FORAGING BOTTLENOSE DOLPHINS, TURSIOPS TRUNCATUS

\author{
By \\ Douglas Paul Nowacek \\ B.A., Ohio Wesleyan University, 1991
}

Submitted in partial fulfillment of the requirements for the degree of

DOCTOR OF PHILOSOPHY

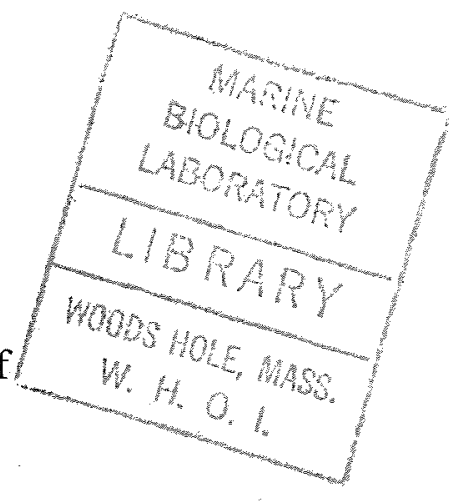

at the

\author{
MASSACHUSETTS INSTITUTE OF TECHNOLOGY \\ and the
}

WOODS HOLE OCEANOGRAPHIC INSTITUTION

September 1999

(C) 1999 Douglas P. Nowacek. All rights reserved.

The author hereby grants to MIT and WHOI permission to reproduce paper and electronic copies of this thesis in whole or in part and to distribute them publicly.

Signature of Author

Joint Program in Biological Oceanography Massachusetts Institute of Technology/ Woods Hole Oceanographic Institution

September 1999

Certified by

Peter Lloyd Tyack Thesis Supervisor

Accepted by

Mark E. Hahn

Chair, Joint Committee on Biological Oceanography Massachusetts Institute of Technology/

Woods Hole Oceanographic Institution 


\title{
SOUND USE, SEQUENTIAL BEHAVIOR AND ECOLOGY OF FORAGING BOTTLENOSE DOLPHINS, TURSIOPS TRUNCATUS
}

\author{
by \\ Douglas Paul Nowacek \\ Submitted in partial fulfillment of the requirements for the degree of Doctor of \\ Philosophy at the Massachusetts Institute of Technology and the Woods Hole \\ Oceanographic Institution
}

\begin{abstract}
Odontocetes are assumed to use echolocation for navigation and foraging, but neither of these uses of biosonar has been conclusively demonstrated in free-ranging animals. Many bats are known to use echolocation throughout foraging sequences, changing the structure and timing of clicks as they progress towards prey capture. For odontocetes, however, we do not know enough about their foraging behavior to describe such sequences. To conduct detailed behavioral observations of any subject animal, the observer must be able to maintain continuous visual contact with the subject for a period commensurate with the duration of the behavior(s) of interest. Behavioral studies of cetaceans, which spend approximately $95 \%$ of their time below the water's surface, have been limited to sampling surface behavior except in special circumstances, e.g. clearwater environments, or with the use of technological tools. I addressed this limitation through development of an observation platform consisting of a remote controlled video camera suspended from a tethered airship with boat-based monitoring, adjustment, and recording of video. The system was used successfully to conduct continuous behavioral observations of bottlenose dolphins in the Sarasota Bay, FL area. This system allowed me to describe previously unreported foraging behaviors and elucidate functions for behaviors already defined but poorly understood. Dolphin foraging was modeled as a stage-structured sequence of behaviors, with the goal-directed feeding event occurring at the end of a series of search, encounter, and pursuit behaviors. The behaviors preceding a feeding event do not occur in a deterministic sequence, but are adaptive and plastic. A single-step transition analysis beginning with prey capture and receding in time has identified significant links between observed behaviors and demonstrated the stagestructured nature of dolphin foraging. Factors affecting the occurrence of specific behaviors and behavioral transitions include mesoscale habitat variation and individual preferences.
\end{abstract}

The role of sound in foraging, especially echolocation, is less well understood than the behavioral component. Recent studies have explored the use of echolocation in captive odontocete foraging and presumed feeding in wild animals, but simultaneous, detailed behavioral and acoustic observations have eluded researchers. The current study 
used two methods to obtain acoustic data. The overhead video system includes two towed hydrophones used to record 'ambient' sounds of dolphin foraging. The recordings are of the 'ambient' sounds because the source of the sounds, i.e. animal, could not be localized. Many focal follows, however, were conducted with single animals, and from these records the timing of echolocation and other sounds relative to the foraging sequence could be examined. The 'ambient' recordings revealed that single animals are much more vocal than animals in groups, both overall and during foraging. When not foraging, single animals vocalized at a rate similar to the per animal rate in groups of $\geq 2$ animals. For single foraging animals, the use of different sound types varies significantly by the habitat in which the animal is foraging. These patterns of use coupled with the characteristics of the different sound types suggest specific functions for each. The presence of multiple animals in a foraging group apparently reduces the need to vocalize, and potential reasons for this pattern are discussed. In addition, the increased vocal activity of single foraging animals lends support to specific hypotheses of sound use in bottlenose dolphins and odontocetes in general. The second acoustic data collection method records sounds known to be from a specific animal. An acoustic recording tag was developed that records all sounds produced by an animal including every echolocation click. The tag also includes an acoustic sampling interval controller and a sensor suite that measures pitch, roll, heading, and surfacing events. While no foraging events occurred while an animal was wearing an acoustic data logger, the rates of echolocation and whistling during different activities, e.g. traveling, were measured.

Thesis supervisor: Peter Lloyd Tyack, Senior Scientist 


\section{Acknowledgements}

I will do my best to properly acknowledge and thank all the people who have provided the assistance, inspiration, and instruction I have received during six years of graduate school, but my gratitude requires more than a few words. First to Peter, my deepest thanks and admiration. It seems almost inconceivable that one person can possess so much optimism and enthusiasm in all aspects of life and research. Despite claims that even he would not have attempted a field project as complicated and risky as mine, it was his tacit confidence and not so tacit optimism that made it possible for me to pursue this project. From the beginning he encouraged me to define interesting and compelling research goals, and then to pursue the methods necessary to attain them. Finally, in the later years of my graduate career, I learned to be a conscientious and thorough scientist by watching as he led projects undaunted by the pressures from both sides of the pendulum.

Next to my committee. Despite the difficulties of getting all of them in the same room at the same time, each gave very insightful and thoughtful input throughout my work. Cindy Moss provided unique suggestions about the sensory integration that dolphins might use based on the abilities of foraging echolocating bats. Dan Rubenstein's provocative discussions regarding behavioral ecology were the inspiration for some of the most revealing results of my work. His almost clairvoyant insights and uncanny ability to convey his ideas were remarkable. John Leonard, despite joining the committed quite late, challenged me to integrate the ideas of a sonar engineer with the abilities of a dolphin which forced me to think with a novel perspective regarding how dolphins use echolocation. Andy Read has been and continues to be a model for me. $\mathrm{He}$ has demonstrated what it is to be an excellent scientist, mentor, and colleague. Andy also has shown me the importance of applying and relating scientific results in a way that benefits both the scientific and non-scientific communities. Randy Wells has been a consistent and generous source of not only scientific discussion and challenge, but he also provided endless support and confidence in the field so that I could pursue that science. Randy's commitment to the dolphins and their well being as well as to our pursuit to understand them is genuinely admirable and an inspiration to me. Michael Scott and Blair Irvine, although not official committee members, provided invaluable insights based on their unique historical perspective of the Sarasota dolphins. They, too, are inspirational in their commitment to the dolphins.

Next I would like to thank all those people in Sarasota without whom I could never have even attempted this project. First among that group is Sue Hofmann. Sue taught me how to drive a boat, navigate the Sarasota waters, and take good photo identification pictures. She also provided an invaluable link to the rest of Mote Marine Lab which was crucial for logistical support as well as for getting on a softball team. Without the space to thank each of the following SDRP/Mote crew individually I owe unique gratitude to each of these people: Kim Bassos-Hull, Howard Rhinehart, Pete Hull, 
Kara Buckstaff, Anna Seles, Joe Nickelson, Hartley Haines, Jay Gorzelany, Caryn Weiss, and Edward (lanyard boy) Owen. An essential part of the Sarasota crew was the cadre of interns that worked through long hot hours on the boat as well as the monotonous hours in front of a computer entering data or in front of a video screen cataloging video tape. So I am indebted to following excellent crew: Erin Kaman, Scott Rifkin, Nikki Hackendahl, Aaron Rundus, Dan Dau, Jennifer Goldman, Liz Johnstone, Brian Lewis, and Patrick Martone. Eric Hendricks, Jim Rohr, and Greg Anderson brought an early version of the overhead video system to Sarasota, and I am grateful to them for getting things off the ground and for loaning me the aerostat. Finally, the seasonal crew in Sarasota that provided intellectual insight and diversity, logistical support, and good times I will not soon forget: Ann Pabst, Bill McLellan, Laela Sayigh, Alex Bocconcelli, James Thorson, Bill Carr, Jeff Stover, Ralph Piland, and the Tequila Fairy.

The acoustic data logger was very much a collaborative effort, and it would not have happened without the commitment and dedication of primarily two groups. First, Trac Pac ${ }^{\circledR}$ in the persons of Frank Deckert and Forrest Townsend, both of whom gave up weekends to highly productive shop time in developing the package to carry the data logger. I appreciate their time, ingenuity, and hospitality. At WHOI I received extremely generous contributions of time and resources from the Advanced Engineering Lab, specifically from Lee Freitag, Matt Grund, Keith von der Hydt, Paul Bouchard, Jim Cooper, and Jim Partan. I am most indebted to my tagging partner in crime and coffee Mark Johnson. Mark has been a completely invaluable combination of friend, confidante, mentor, electronics expert, and tagging enthusiast. Without his expertise and extremely generous gifts of time and enthusiasm the data logger would never have happened.

My knowledge of the biology and ecology of marine mammals would not be nearly as thorough or interesting without the often casual but always fruitful discussions with Bill Watkins. Darlene Ketten also provided thoughtful insights during our periodic impromptu hallway meetings. Thanks to Mary Ann Daher for my sporadic requests for tidbits of data and history. Amy Samuels has been very generous both personally and professionally, always a patient and extremely helpful sounding board in developing behavioral sampling protocols and subsequent interpretations of the data. Thanks to Vincent Janik who happened along at a fortuitous time in my thesis writing, always willing to chat about data interpretations and providing excellent literature background. A special debt of gratitude to Andy Solow who always made himself available to sort through data analyses and statistical testing. On to the Tyack lab students. Patrick Miller has been an good friend and colleague since he arrived at WHOI. From field projects to lab programming and analyses, Patrick has always been willing to lend an able hand, cycloptopus will happen one day. Thanks to Debby Fripp, Rebecca Thomas, Stephanie Watwood, and Susan Parks for making the Tyack lab a dynamic place to work. Thanks also to my Woods Hole friends/fellow grad students: Mike Atkins, Ewann AgenbroadBerntson, Nico Biasonni, Rebecca Green, Mark Hill, Brenda Jensen, Jennifer JoySchlezinger, Ee Lin Lim, Nicole Poulton, Gorka Sancho, and Gaspar Taroncher- 
Oldenburg. The final Woods Hole thanks goes to Jake Peirson, Stella Callagee, Julia Westwater, and the rest of the education office.

To my family, old and new, your support, confidence, and honest interest in me and my work made the rough times bearable and the good times even more rewarding. To my parents, you have always inspired my curiosity and instilled the confidence and motivation to pursue what most intrigues me, thank you. To Stephanie, your love, inspiration, and support are absolutely the most important things I have.

Finally, a respectful thanks to the Sarasota dolphins, especially those that allowed me a glimpse of their lives, or in other words tolerated the shadow of the balloon: FB03, FB11, FB28, FB38, FB54, FB65, FB75, FB79, FB83, FB92, F101, F118, F138, F155, F157, and two unknown 'Tampa' animals.

This work was supported by the Education Office of the Woods Hole Oceanographic Institution, two grants from the Rinehart Coastal Research Center, the Ocean Ventures Fund, 'WHOI Sea Grant, ONR Grant \#N00014-94-1-0692 to P. Tyack, and a Graduate Fellowship from the Office of Naval Research. This research was conducted under the general authorization marine mammal permit to R. S. Wells 


\section{List of Figures}

Figure 2.1. Map of study area.

Figure 3.1. Inter-hydrophone calibration. Both the reference (a) and test (b) hydrophones are $1 \mathrm{~m}$ in front of the animal. A click train and a 'zoomed-in' waveform of one click are shown for (a) the reference hydrophone (top) and (b) test hydrophone. Average relative amplitudes ( $\mathrm{dB}$ referenced to $1 \mathrm{~V}$ ) are shown with figure sub-label, (a) or (b), for clicks recorded at the corresponding location.

Figure 3.2. Click waveforms recorded by (a) the reference hydrophone at the $1 \mathrm{~m}$ reference position, and by (b) the test sensor at test position \#1, the melon. A click train and a 'zoomed-in' waveform of one click are shown for (a) the reference hydrophone (top) and (b) test hydrophone. Average relative amplitudes ( $\mathrm{dB}$ referenced to $\mathrm{VV}$ ) are shown with figure sub-label, (a) or (b), for clicks recorded at the corresponding location.

Figure 3.3. Click waveforms recorded by (a) the reference hydrophone at $1 \mathrm{~m}$, and (b) the test hydrophone at test position \#2, the body at the base of the dorsal fin. A click train and a 'zoomed-in' waveform of one click are shown for (a) the reference hydrophone (top) and (b) test hydrophone. Average relative amplitudes ( $\mathrm{dB}$ referenced to $1 \mathrm{~V}$ ) are shown with figure sub-label, (a) or (b), for clicks recorded at the corresponding location.

Figure 3.4. Two sets of click waveforms recorded by the reference hydrophone at $1 \mathrm{~m}$ and test hydrophone at test position \#3, on the dorsal fin. A click train and a 'zoomed-in' waveform of one click are shown in (a) and (b). In (a) and (b) the top set of two waveforms (click train and 'zoomed-in' click) was recorded by the reference hydrophone, and the lower set by the test hydrophone. Average relative amplitudes (dB referenced to $1 \mathrm{~V}$ ) are shown with figure sub-label, (a) or (b), for clicks recorded at the corresponding location. The relative amplitude for the reference hydrophone is shown first.

Figure 3.5. Spectral comparison of signals from the reference hydrophone and test hydrophone at position \#1, the melon. The power spectral density of the test hydrophone relative to the reference (top), the coherence function between the two channels (middle), and the ratio (dBV) of the test hydrophone compared to the reference (bottom). See text for explanation of power spectral density and coherence.

Figure 3.6. Spectral comparison of signals from the reference hydrophone and test hydrophone at position \#2, on the body at the base of the dorsal fin. The power spectral density of the test hydrophone relative to the reference (top), the coherence function between the two channels (middle), and the ratio (dBV) of the test hydrophone compared to the reference (bottom). See text for explanation of power spectral density and coherence. 
Figure 3.7. Spectral comparison of signals from the reference hydrophone and test hydrophone at position \#3, on the dorsal fin. The power spectral density of the test hydrophone relative to the reference (top), the coherence function between the two channels (middle), and the ratio (dBV) of the test hydrophone compared to the reference (bottom). See text for explanation of power spectral density and coherence.

Figure 3.8a. Click rate record for F111. Mean clicks/second and standard deviation are shown in the center of the figure for the entire 112-minute record as well as in the top corners for the periods before and after release. 'Release time' is indicated by the red line bounded with asterisks.

Figure 3.8b. Whistle rate record for F111 (top) and samples of whistles from pre-release (lower left) and 1-hour post-release (bottom right). Mean whistles/min and standard deviation are shown in the center of the top figure for the entire 112-minute record as well as in the top corners for the periods before and after release. 'Release time' is indicated by the red line bounded with asterisks. The two whistle samples show that F111 used time-warped versions of her signature whistle pre and post-release.

Figure 3.8c. Log-log survival plot of all inter-click intervals for F111. For each ICI duration on the abscissa the value of the ordinate indicates the number of ICI's recorded that are of that duration and longer.

Figure 3.9a. Click rate record for FB03. Mean clicks/second and standard deviation are shown in the center of the figure for the entire 92-minute record as well as in the top corners for the periods before and after release. 'Release time' is indicated by the red line bounded with asterisks.

Figure 3.9b. Whistle rate record for FB03. The main figure shows only a short part of the entire record around the time of release for better histogram resolution. The inset shows the entire record; FB03 did not whistle after the few emitted around the time of release. Mean whistles/min and standard deviation are shown in the center of the figure for the entire 92-minute record as well as in the top corners for the periods before and after release. 'Release time' is indicated by the red line bounded with asterisks.

Figure 3.9c. Log-log survival plot of all inter-click intervals for FB03. For each ICI duration on the abscissa the value of the ordinate indicates the number of ICI's recorded that are of that duration and longer.

Figure 3.10a. Click rate record for F115 segment 1. Mean clicks/second and standard deviation are shown in the center of the figure for the 20-minute segment as well as in the top corners for the periods before and after release. 'Release time' is indicated by the red line bounded with asterisks.

Figure 3.10b. Whistle rate record for F115 segment 1. Mean whistles/minute and standard deviation are shown in the center of the figure for the 20 -minute segment as well 
as in the top corners for the periods before and after release. 'Release time' is indicated by the red line bounded with asterisks.

Figure 3.10c. Click rate record for F115 segment 2. Mean clicks/second and standard deviation are shown in the center of the figure for the 20 -minute segment. This segment began 1 hour post-release.

Figure 3.10d. Click rate record for F115 segment 3. Mean clicks/second and standard deviation are shown in the center of the figure for the 20 -minute segment. This segment began 2 hours post-release.

Figure 3.10e. Log-log survival plot of all inter-click intervals for F115. For each ICI duration on the abscissa the value of the ordinate indicates the number of ICI's recorded that are of that duration and longer.

Figure 3.11a. Click rate record for TNLV segment 1. Mean clicks/second and standard deviation are shown in the center of the figure for the 10-minute segment. 'Release time' is indicated by the red line bounded with asterisks.

Figure $3.11 \mathrm{~b}$. Click rate record for TNLV segment 2. Mean clicks/second and standard deviation are shown in the center of the figure for the 5 -minute segment.

Figure 3.11c. Click rate record for TNLV segment 3. Mean clicks/second and standard deviation are shown in the center of the figure for the 5-minute segment.

Figure 3.11d. Log-log survival plot of all inter-click intervals for TNLV. For each ICI duration on the abscissa the value of the ordinate indicates the number of ICI's recorded that are of that duration and longer.

Figure 3.12a. Click rate record for FB09 segment 1. Mean clicks/second and standard deviation are shown in the center of the figure for the 20 -minute segment as well as in the top corners for the periods before and after release. 'Release time' is indicated by the red line bounded with asterisks.

Figure 3.12b. Whistle rate record for FB09 for segment 1. Mean whistles/minute and standard deviation are shown in the center of the figure for the 20 -minute segment as well as in the top corners for the periods before and after release. 'Release time' is indicated by the red line bounded with asterisks.

Figure 3.12c. Click rate record for FB09 segment 2. Mean clicks/second and standard deviation are shown in the center of the figure for the 16-minute segment as well as in the top corners for the periods before and after release.

Figure 3.12d. Log-log survival plot of all inter-click intervals for FB09. For each ICI duration on the abscissa the value of the ordinate indicates the number of ICI's recorded that are of that duration and longer. 
Figure 3.13a. Click rate record for F149 segment 1. Mean clicks/second and standard deviation are shown in the center of the figure for the 10-minute segment. Note: F149 was not sampled prior to release.

Figure 3.13b. Whistle rate record for F149 segment 1. Mean whistles/minute and standard deviation are shown in the center of the figure for the 10-minute segment. Note: F149 was not sampled prior to release.

Figure 3.13c. Click rate record for F149 segment 2. Mean clicks/second and standard deviation are shown in the center of the figure for the 5-minute segment.

Figure 3.13d. Whistle rate record for F149 segment 2. Mean whistles/minute and standard deviation are shown in the center of the figure for the 5-minute segment.

Figure 3.13e. Log-log survival plot of all inter-click intervals for F149. For each ICI duration on the abscissa the value of the ordinate indicates the number of ICI's recorded that are of that duration and longer.

Figure 4.1a. Habitat use by focal animals.

Figure 4.1b. Habitat use by focal animals continued.

Figure 4.2. 'Root' from the perspective of an underwater viewer observing the animals at the bottom.

Figure 4.3. 'Kerplunk' as it would look to an observer looking across the surface of the water. The three drawings (top to bottom) show the chronological progression of the behavior.

Figure 4.4. 'Fish whack' seen from the overhead perspective, i.e. the animals are swimming on their sides so an overhead observer sees a lateral view of the animals. As shown, the whack can be either a dorsal or ventral thrust of the flukes.

Figure 4.5 'Side-swimming' as seen from the overhead perspective (top drawing) and from the perspective of an observer underwater at the same depth as the animal (bottom two drawings).

Figure 4.6 'Pinwheel' as seen from overhead. The key to visualizing this behavior is to understand that in the figure all three drawings show the right side of the animal. From overhead, the same lateral side of the animal is in view throughout the behavior.

Figure 4.7. Behavioral sequences leading to successful captures. Each transition represents a single state step between behaviors. Sequences were traced backwards to either non-foraging behavior or another capture event. Abbreviations are as follows: cp- 
capture; pw-pinwheel; ac-accelerate; fw-fish whack; rt-root; ss-side swim; kp-kerplunk; sc-scan; ec-echolocate; wh-whistle; pp-pop; df-drift; bd-bottom disturbance/bubble cloud.

Figure 4.8. Bifurcation diagram of foraging sequences. Foraging sequences from Figure 4.7 have been categorized into the major categories displayed. The numbers shown on the branches indicate the number of occurrences of each pathway. The numbers in parentheses at the end of each pathway indicate the average number of steps to prey capture for that pathway.

Figure 4.9. Behavioral transitions leading to prey capture: state-lag $z$-scores. The different colored boxes for the various behaviors (Figures 4.9-12) are meant to group behaviors relative to their minimum number of steps (i.e. transitions) away from capture.

Figure 4.10. Behavioral transitions leading to prey capture: state-lag $z$-scores for sand feeding.

Figure 4.11. Behavioral transitions leading to prey capture: state-lag z-scores seagrass feeding.

Figure 4.12. Behavioral transitions leading to prey capture: state-lag $z$-scores for seagrass edge feeding.

Figure 4.13. Rate of occurrence of foraging behaviors during general activities.

Figure 5.1a. An example of echolocation clicks recorded by the two-hydrophone towed system. The relative level of the sounds is indicated by the color scale, which maps to the color bar on the right.

Figure 5.1b. An example of whistles recorded by the two-hydrophone towed system. The relative level of the sounds is indicated by the color scale, which maps to the color bar on the right.

Figure 5.1c. An example of pops recorded by the two-hydrophone towed system. The relative level of the sounds is indicated by the color scale, which maps to the color bar on the right.

Figure 5.2a. Rate of sound production by group size for all behaviors.

Figure $5.2 \mathrm{~b}$. Rate of sound production by group size during foraging.

Figure 5.3. Sound production rate for single vs. multiple animal groups across all behaviors.

Figure 5.4. Sound production rate and foraging status for single vs. multiple animal groups. 
Figure 5.5. Sound production rates for single foraging animals in the three primary habitats.

Figure A1.1. Schematic drawing of overhead video-ambient acoustic observation system.

Figure A2.1. Data logger attached with a Trac $\mathrm{Pac}^{\circledR}$.

Figure A2.2. Echolocation clicks recorded by the data logger's 'ambient' hydrophone.

Figure A2.3. Whistles recorded by the data logger's 'ambient' hydrophone. 


\section{List of Tables}

Table 3.1. Tally of acoustic data logger attachments.

Table 4.1. Focal animals included in the study.

Table 4.2. Total behavioral pair transitions tallied for the $z$-score analyses.

Table 4.3. Negative $z$-scores greater than -1.96 . 'Target' and 'Given' are described in Section 4.2.3.

Table 4.4. P-values (from adjusted residuals) for the use of behaviors by individuals.

Table 4.5. Example of individual $2 \times 2$ contingency table analyses for the habitat-specific occurrence of foraging behaviors.

Table 4.6. $\chi^{2} \mathrm{P}$-values comparing each foraging behavior with all others across habitats.

Table 4.7 Distance of focal animal's nearest neighbor and occurrence of foraging behaviors. Data for females with dependent calves have been removed.

Table 4.8. $\chi^{2}$ P-values for $2 \times 2$ contingency table analyses of foraging behavior and NND.

Table 5.1. Focal animals included in the study.

Table 5.2. Occurrence of three sound types in single vs. multi-animal groups.

Table 5.3. Occurrence of sounds in single vs. multi-animal groups by behavior. 'Foraging' includes only those sounds recorded during previously defined foraging behaviors.

Table 5.4. Habitat-specific occurrences of sounds across all behaviors.

Table 5.5. Habitat-specific occurrences of sounds by single foraging animals.

Table A1.1. Summary of behavioral observation techniques: advantages, disadvantages, applications and references (note: references are not meant to be exhaustive, simply representative). 


\section{Table of Contents}

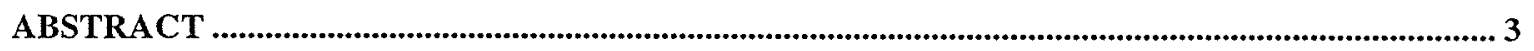

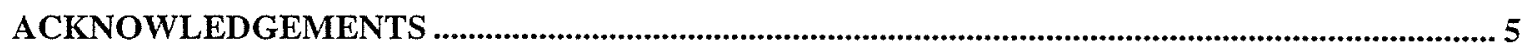

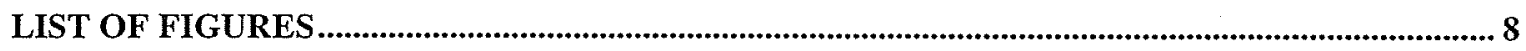

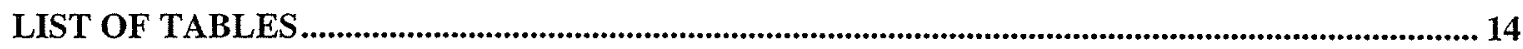

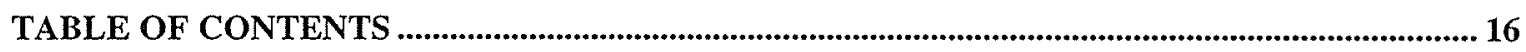

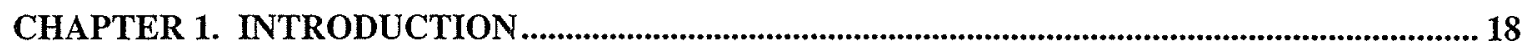

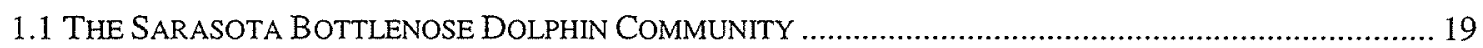

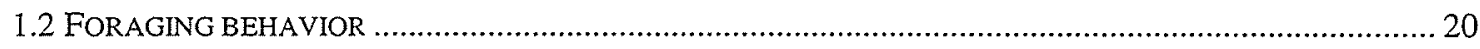

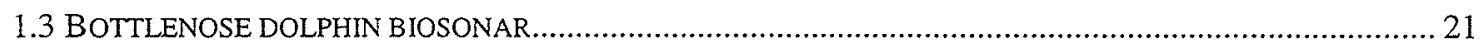

1.4 INTRODUCTION TO THESIS RESEARCH

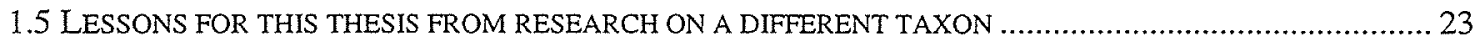

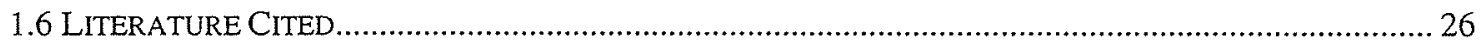

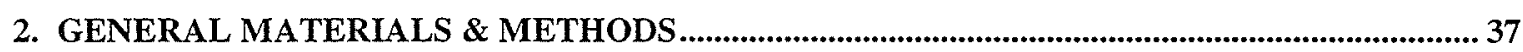

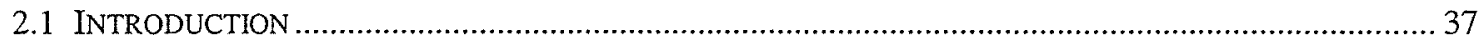

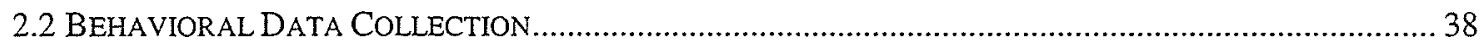

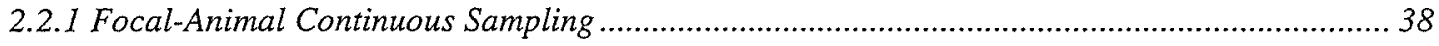

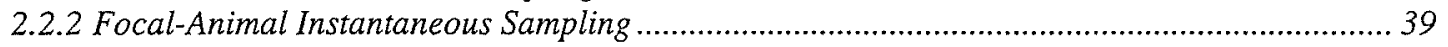

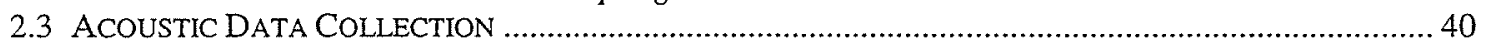

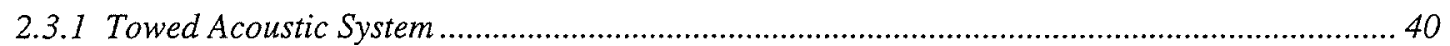

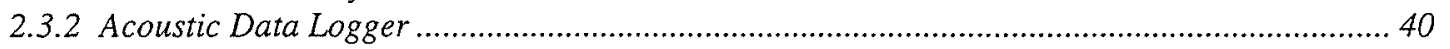

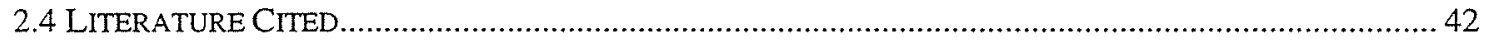

CHAPTER 3. ACOUSTIC ACTIVITY OF FREE-RANGING BOTTLENOSE DOLPHINS ........... 44

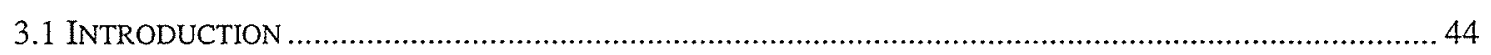

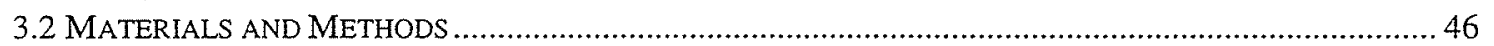

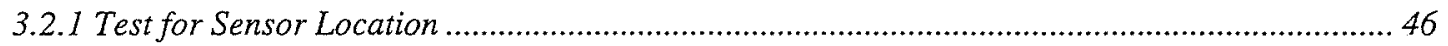

3.2.2 Acoustic Data Logger: Configuration and Analysis of Recordings ........................................... 48

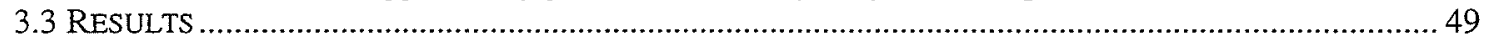

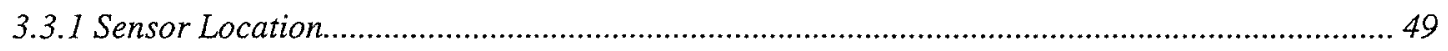

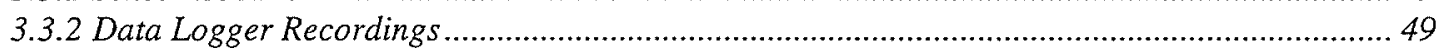

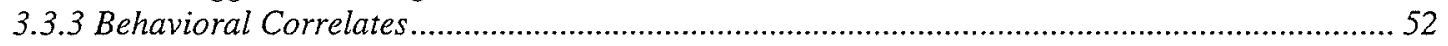

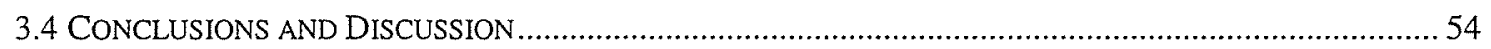

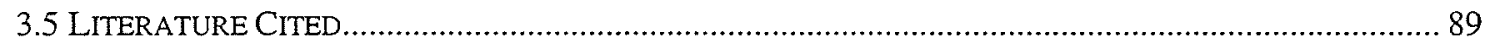

4 FORAGING BEHAVIOR OF SARASOTA BOTTLENOSE DOLPHINS..................................... 93

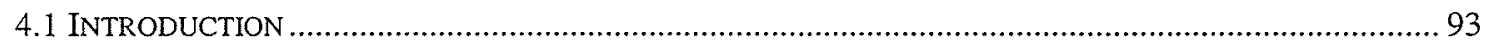

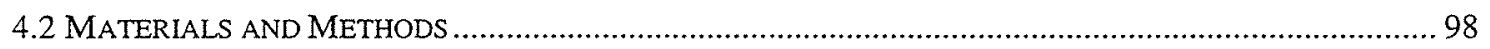

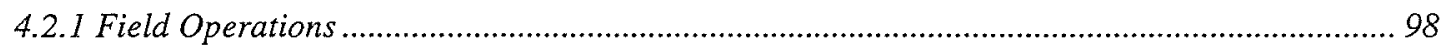

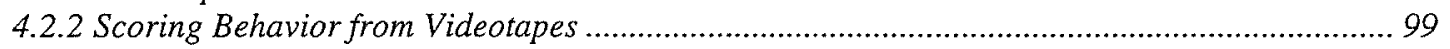

4.2.3 Analysis of Behavioral Sequences.......................................................................................... 101

4.2.4 Analysis of Individual/Habitat Specific Use of Foraging Behaviors....................................... 103

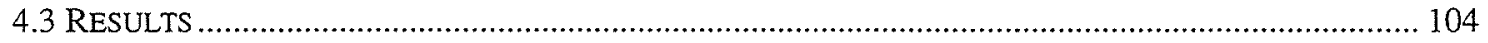

4.3.1 Focal animals: profiles and data collected ....................................................................... 104

4.3.2 Definitions and Descriptions of Observed Behaviors: States $(S)$ and Events $(E)$................... 105

4.3.3 The Sequential Nature of Dolphin Foraging.................................................................... 111

4.3.4 Influence of Individuals and Habitats on Observed Behavioral Patterns ............................. 113 
4.3.5 Nearest Neighbor Distance and Occurrence of Foraging Behaviors .................................. 115

4.3.6 Foraging behaviors occurring during other activities..................................................... 117

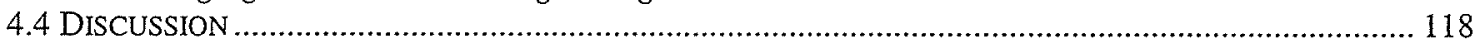

4.4.1 Foraging Behavior Sequences .................................................................................... 118

4.4.2 Distinguishing Characteristics of Foraging Behaviors: Individual and Habitat Influences and

Indications for Distinct Foraging Strategies......................................................................... 120

4.4.3 Nearest Neighbor Distance and Foraging Behavior....................................................... 124

4.4.4 New insights into Sarasota dolphin foraging ................................................................. 126

4.4.5 Comparison of boat-based vs. overhead observations: an aide for the boat-based observer.. 127

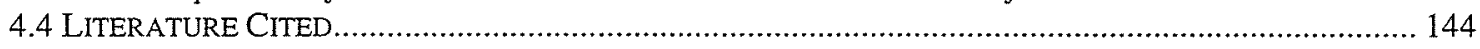

CHAPTER 5. AMBIENT ACOUSTICS OF FORAGING BOTTLENOSE DOLPHINS ................ 151

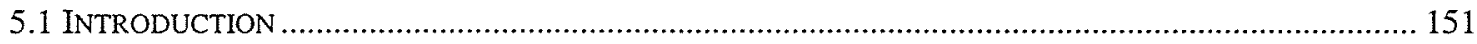

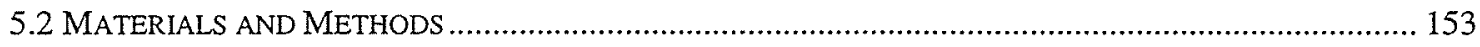

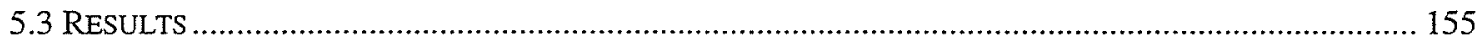

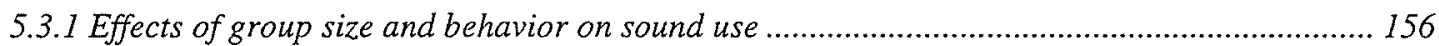

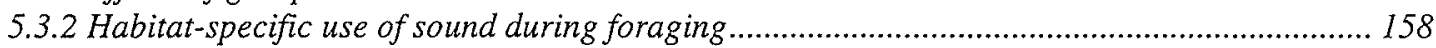

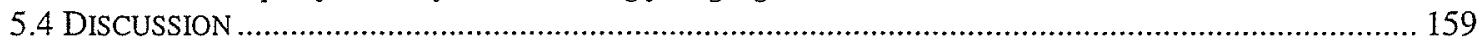

5.4.1 Use of Sounds by Single vs. Multiple Animal Groups ..................................................... 159

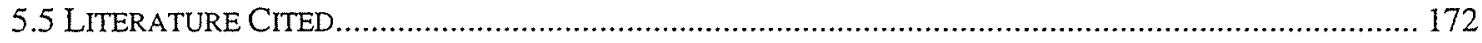

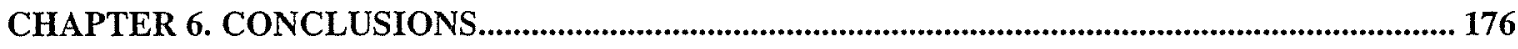

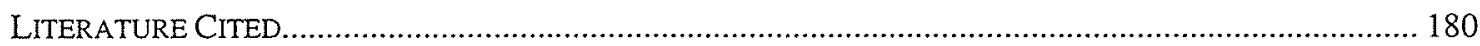

APPENDIX 1. OVERHEAD VIDEO/UNDERWATER ACOUSTIC OBSERVATION PLATFORM

181

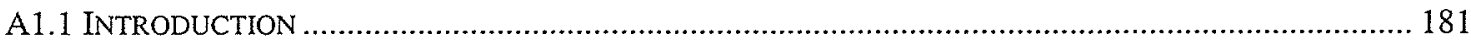

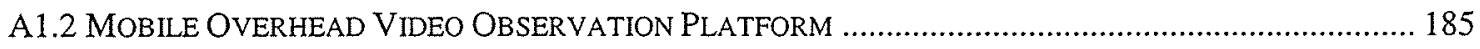

A1.2.1 System Configuration and Operation ………................................................................ 185

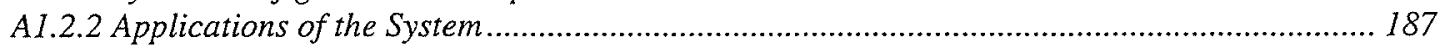

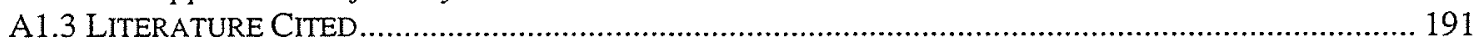

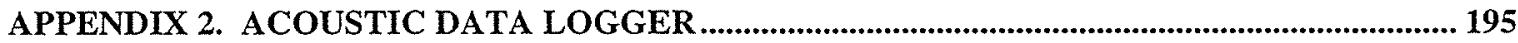

REPRINTED WITH PERMISSION FROM THE JOURNAL OF THE ACOUSTICAL SOCIETY OF AMERICA ........... 195 


\section{Chapter 1. Introduction}

All animals must find and select food to survive, and most predatory animals must also pursue and capture their prey. Finding and obtaining food is one of the most basic biological and ecological challenges animals face. A successful predator must develop foraging strategies that integrate sensory information with motor behaviors. Research on terrestrial predators has focused on the sequential stages of predation: detection, selection, pursuit, and capture (Stephens \& Krebs, 1986). In so doing, these efforts have successfully characterized the motor behaviors, and have provided an intimate understanding of the foraging ecology of many species (Evans, 1982; Focardi, Marcellini \& Montanaro, 1996; Goss-Custard et al., 1995; Hemmi \& Menzel, 1995; Jones, 1990; Rapaport, 1998; Schmid-Hempel, Kacelnik \& Houston, 1985; Yamagiwa \& Mwanza, 1994). For marine mammals, however, this knowledge is still at a relatively primitive, descriptive level. With regard to sensory systems, sound is presumably an important sense for predatory marine mammals due to its properties in water and the often-poor visibility in many habitats. Odontocete echolocation, for example, is excellent for target detection, ranging, and discrimination (Au, 1993). While we know a great deal about the capabilities of the echolocation system from studies of captive dolphins echolocating on artificial targets, only circumstantial evidence exists for its use by wild foraging animals (Miller et al., 1995). Norris and Møhl (1983) hypothesized that dolphins might use high intensity clicks to stun their fish prey. While there has been no repeatable evidence to support this hypothesis, high-intensity, click-like sounds could be used simply to startle or otherwise flush fish prey from their refuges. Some dolphin-produced sounds possess acoustic energy and characteristics sufficient to make this function theoretically possible (Akamatsu et al., 1996; Blaxter, Gray \& Denton, 1981; Blaxter \& Hoss, 1981; Connor \& Smolker, 1996; dos Santos et al., 1990; Eaton, Bombardieri \& Meyer, 1977).

The bottlenose dolphin, Tursiops truncatus, is an excellent species to study the specific, sequential motor behaviors and use of sound during foraging. These animals inhabit shallow, coastal waters in which they can be observed continuously under certain 
circumstances (Connor, Smolker \& Richards, 1992; Nowacek et al., 1995). In addition, we have extensive information about the distribution, anatomy, life history, diet, and general sound production of this species (Caldwell \& Caldwell, 1965; Cockcroft \& Ross, 1990; Gunter, 1942; Herman \& Tavolga, 1980; Hohn et al., 1989; Irvine et al., 1981; Irvine \& Wells, 1972; Kemp, 1949; Kleinenberg, 1938; Leatherwood, Deerman \& Petter, 1978; Mead \& Potter, 1990; Popper, 1980; Scott, Wells \& Irvine, 1990; Tsalkin, 1940; Van Waerebeek et al., 1990; Wells, 1991; Wells \& Scott, 1999). While some aspects of bottlenose dolphin biology are relatively well understood, the use of sound, especially biosonar, in wild dolphins is not well characterized. One of the commonly assumed functions of biosonar is to find and secure food, but this hypothesis has been tested only in captivity (Akamatsu et al., 1994; Verfuss \& Schnitzler, 1995) or during presumed feeding in wild odontocetes that are only distantly related to dolphins (Miller et al., 1995). To investigate whether and, if so, how dolphins use biosonar and/or other sounds during foraging requires continuous observations of foraging dolphins. For bottlenose dolphins our knowledge of their foraging behavior is limited to qualitative observations, but these descriptive reports do provide a basis for quantitative study of sequential foraging behavior. This thesis focused on two primary questions: 1) what are the specific behaviors bottlenose dolphins use to find, pursue, and capture their prey?; and 2) how, if at all, do they use sound as part of this process? Chapter 2 introduces the methods used to gather data. Chapter 3 provides a glimpse into the acoustic activity of individual freeranging dolphins through the use of a novel research tool. Chapter 4 reports the sequential behavior and ecology of foraging dolphins, and Chapter 5 explores the ecology of sound use during foraging.

\section{I The Sarasota Bottlenose Dolphin Community}

The research reported in this thesis was conducted almost exclusively on the resident bottlenose dolphins inhabiting the waters of Sarasota Bay, FL. This dolphin community has been the focus of a long-term study that began in 1970 with an initial focus to examine ranging and social patterns of free ranging bottlenose dolphins (Irvine \& Wells, 1972). Since that time Blair Irvine, Randall Wells, Michael Scott, and many collaborators have studied many aspects of this community (Barros \& Wells, 1998; 
Duffield \& Wells, 1991; Hohn et al., 1989; Irvine et al., 1981; Read et al., 1993; Sayigh et al., 1990; Sayigh et al., 1995; Waples et al., 1995; Wells, 1991; Wells, Irvine \& Scott, 1980; Wells, Scott \& Irvine, 1987). These extensive research efforts have revealed the social and community structure, matrilineal relationships, home range sizes, activity budgets, habitat use patterns, and important prey species for the Sarasota bottlenose dolphins.

A temporary capture and release program has provided a great deal of information about wild dolphins that could not be obtained through simple observations. During most years from 1984-1999, small numbers of dolphins were encircled with a seine net deployed by a commercial fishing boat. The dolphins swim freely in the net corral, and are then restrained one at a time to be measured, sampled; sometimes marked, and then released. Samples collected included morphometrics, blood for health assessment, blood for genetic and contaminant analyses, a tooth for age determination in cases when age is not known from observations (Hohn et al., 1989), and recordings acoustic activity. This project has yielded basic information about the animals including sex, age, genetic relationships, and acoustic activity and repertoires.

\subsection{Foraging behavior}

The foraging behavior of wild bottlenose dolphins is known primarily from descriptive reports of entire dolphin groups (Bel'kovich et al., 1991; Caldwell \& Caldwell, 1972; Hamilton \& Nishimoto, 1977; Leatherwood, 1975; Shane, 1990; Shane, Wells \& Würsig, 1986; Tayler \& Saayman, 1972), although a few reports have described individual hunting techniques (Bel'kovich et al., 1991; Hoese, 1971; Leatherwood, 1975; Petricig, 1995; Wells et al., 1987). These descriptive accounts do not provide quantitative analyses of specific behaviors or sequences, but they do reveal the variety and plasticity of bottlenose dolphin foraging behavior and establish a basis for more detailed study. Tinbergen (1951) discusses the plasticity and adaptiveness of what Craig (1918) called 'appetitive' behaviors, or those behaviors leading up to a consummatory act such as feeding. Tinbergen (1951) summarizes a consummatory act as being, “... 
relatively simple; at its most complex, it is a chain of reactions, each of which may be a simultaneous combination of a taxis and a fixed pattern" (p. 106). In contrast Tinbergen describes appetitive behaviors as, ".... conglomerate of many elements of very different order, of reflexes, of simple patterns like locomotion, of conditioned reactions, of 'insight' behaviour, and so on" (p.106). The discrimination between these two classes of behavior is only the first phase in analyzing sequences of behavior like foraging (Tinbergen, 1951).

One manifestation of appetitive behaviors is the presence of foraging differences or specializations within species, or even within a population (Goss-Custard \& Le V dit Durell, 1983; Goss-Custard, Le V dit Durell \& Ens, 1982; Goss-Custard \& Sutherland, 1984; Le V. dit Durell \& Goss-Custard, 1984; Swennen et al., 1983). Individuals of some marine mammal species have also been observed to use varying foraging strategies (Hoelzel, Dorsey \& Stern, 1989; Lopez \& Lopez, 1985; Rogers \& Bryden, 1995; Weinrich, Schilling \& Belt, 1992) including some evidence that these behaviors are transmitted through populations (Lopez \& Lopez, 1985; Weinrich et al., 1992). Evidence presented in Chapter 4 demonstrates that the Sarasota bottlenose dolphins utilize at least three distinct search strategies, and anecdotal observations suggest that at least two of these behaviors may be socially learned.

\subsection{Bottlenose dolphin biosonar}

Research with captive animals echolocating on artificial targets has elucidated the design and operation of the dolphin biosonar system $(\mathrm{Au}, 1993)$. While this research has provided detailed information about the capabilities and limitations of the system, we have virtually no information about how the animals use biosonar in the wild. In the 1950's Schevill and Lawrence conducted the earliest experiments demonstrating high frequency auditory capability in bottlenose dolphins (Schevill \& Lawrence, 1953a; Schevill \& Lawrence, 1953b). Other early studies experimented with a bottlenose dolphin's use of echolocation to find fish (D. Griffin, personal communication 1999;(Kellogg, 1961), but the experimental confirmation of their ability to use 
echolocation to find a target was reported by Norris et al. (1961). Between 1953 and 1993 details of the system have been illustrated through controlled experimentation. The transmit and receive systems (Aroyan, 1990a; Brill \& Harder, 1991; Brill et al., 1988; Cranford, 1988; Norris \& Harvey, 1974) and on-axis beam patterns (Au, Moore \& Pawloski, 1986) are well characterized, although some debate about the specific production mechanism still exists (Aroyan, 1990a; Aroyan, 1990b; Cranford, 1988;

Pilleri, 1990; Reidenberg \& Laitmann, 1988). The debate over production centers on whether clicks are produced by a laryngeal mechanism or by a forced-air, piston mechanism with the dorsal bursae being the origin of the sound. The structure of the clicks including frequency and time characteristics is well known ( $\mathrm{Au}, 1980)$.

Echolocation clicks are short $(50-150 \mu \mathrm{sec})$, broadband $(10-120 \mathrm{kHz})$ sounds, although the primary energy occurs between $40-100 \mathrm{kHz}$. We know the click production patterns for trained dolphins echolocating on artificial targets (Evans \& Powell, 1967; Turl \& Penner, 1989) and the detection (Au, 1993; Au \& Penner, 1981; Au \& Snyder, 1980; Murchison, 1980) and discrimination (Au, 1993; Au \& Turl, 1991; Hammer \& Au, 1980) capabilities.

\subsection{Introduction to thesis research}

The two questions posed in this thesis demand data in two critical areas. First, it was necessary to know with confidence when an animal was foraging, and, if so, in what stage of foraging it was engaged (Stephens \& Krebs, 1986). To obtain this information, the first goal of this study was to identify the specific behaviors dolphins use during the different stages of foraging. Once known, the occurrences of these behaviors were matched with simultaneous acoustic records to attain the second goal of this study, which was to investigate the use of sound during foraging. Obtaining acoustic recordings known to be from a specific individual is a perennial problem for cetacean researchers. This problem was addressed in two ways during the current study. First, a towed hydrophone system similar to that used by Sayigh et al. (1993) was used to record the sounds of single animals (i.e. no other animals within $1 \mathrm{~km}$ ) while foraging. Second, a non-invasive acoustic recording tag was attached to dolphins that were captured, temporarily restrained, and subsequently released and observed. No foraging behaviors 
occurred while an animal was wearing an acoustic recording tag, but the towed hydrophone system produced interesting acoustic data from single foraging animals.

\subsection{Lessons for this thesis from research on a different taxon}

The behavior and acoustic activity of foraging microchiropteran bats illustrate the ecological complexity and richness of a predator-prey system in which echolocation plays a role in foraging. Griffin (1958) reviewed the history of bat echolocation and reported the results of his many insightful experiments into its uses and potential mechanisms. His obstacle avoidance experiments, for example, provided the first conclusive evidence of bats using echolocation for precision flight (Griffin, 1958). These early findings have proven to be extremely robust and have provided a sound foundation for research into the details of the bat auditory (Popper \& Fay, 1995) and perceptual (e.g. (Simmons, Moss \& Ferragamo, 1990) systems. A synergy between laboratory and field studies has proven crucial to gaining an understanding of bat echolocation and foraging. Griffin (1960), for example, recognized the need to confirm field results with careful laboratory experiments despite the strong evidence already collected. This synergy has continued, not only between laboratory and field studies, but also between the relevant disciplines of behavior, psychology, and physiology (see (Grinnel, 1995).

What are the results of this synergistic research? We know the basic patterns of behavior (Faure \& Barclay, 1994; Jones, 1995; Jones et al., 1993; Kalko, 1995; Simmons, Fenton \& O'Farrell, 1979) and echolocation (Barclay, 1986; Britton et al., 1997; Faure \& Barclay, 1994; Jones, 1995; Kalko, Schnitzler \& Grinnel, 1998) bats use to find, pursue, and capture insects. In addition, we have detailed information regarding the problems bats must routinely solve such as the choice of prey items (Emde \& Schnitzler, 1990), the type of signal to use based on the task and the environmental constraints (Barclay, 1986; Neuweiler, 1983; Rydell, 1993; Schnitzler et al., 1994), the acoustic information available in various situations (Kober \& Schnitzler, 1990; Moss \& Zagaeski, 1994), and the way in which bats process echoes (Dear \& Suga, 1995;

Simmons et al., 1990). Finally, and again as a result of both field and laboratory studies, we understand a great deal about the interactions between bats and their prey. Insect prey 
are known to detect and react to bat echolocation both behaviorally and acoustically (Fullard, Fenton \& Simmons, 1979; Fullard, Simmons \& Saillant, 1994; Haskell \& Belton, 1956; Miller, 1983; Miller, 1991; Roeder, 1962; Roeder, 1967; Roeder \& Treat, 1957; Zhantiyev, Lapshin \& Fedorova, 1993), and bats have responded by either catching prey in spite of countermeasures (Miller, Futtrup \& Dunning, 1996), or, more commonly by hunting without the use of echolocation (Fiedler, 1979; Ryan \& Tuttle, 1983; Ryan, Tuttle \& Barclay, 1983; Tuttle \& Ryan, 1981; Tuttle, Ryan \& Belwood, 1985).

The current state of understanding of the function of dolphin biosonar in the wild lags significantly behind our knowledge for bats, but further research may reveal a predator-prey system that is equally as rich. Many fish, for example, can detect and react to frequencies contained in dolphin whistles and echolocation (Astrup \& Mfhl, 1993; Blaxter et al., 1981; Blaxter \& Hoss, 1981; Canfield \& Eaton, 1990; Dunning et al., 1992; Fay, 1988; Mann, Lu \& Popper, 1997; Popper, 1980; Popper \& Fay, 1988; Rogers et al., 1988), and the fish preyed upon by dolphins produce sounds that are likely audible to the dolphins (Barros \& Wells, 1998; Fish \& Mowbray, 1970).

Studying animals in a field setting like Sarasota allows research to address questions regarding the foraging behavior and use of sound by bottlenose dolphins. As discussed above, previous research has identified some specific foraging behaviors, which provides a basis for the detailed study of these behaviors and their ecology. In the shallow waters inhabited by the Sarasota dolphins, not only the end result (i.e. prey capture) but the entire foraging sequence can be observed and quantified. By knowing whether and in what stage of foraging an animal is engaged, behavioral and acoustic questions can be addressed similar to those answered by bat research. Another advantage afforded by the Sarasota venue was the extensive background knowledge of the animals. With this information, questions such as individual foraging preferences and their relationship to preferences of related individuals could be addressed. Finally, the access to temporarily restrained animals to which acoustic recording devices could be attached made possible the initial study of the acoustic activity of free-ranging animals. 
To begin to understand the use of biosonar and other sounds by foraging bottlenose dolphins, this thesis presents data on the occurrence and ecology of the detailed, sequential behaviors that the Sarasota bottlenose dolphins used to search for, pursue, and capture prey. The sounds animals produced while engaged in these behaviors could then be analyzed as being definitively involved in foraging. Moreover, by observing single foraging animals (i.e. no other animals within $1 \mathrm{~km}$ ), specific functions of sounds produced could be explored. To gain further inroads into the specific use of sounds by free-ranging individuals, acoustic data loggers were attached to individuals for whom simultaneous behavioral data were also collected. Further studies using the data gathered and tools developed for this thesis will doubtless increase our understanding of the behavioral and acoustic ecology of foraging bottlenose dolphins. 


\subsection{Literature Cited}

Akamatsu, T., Hatakeyama, Y., Kojima, T. \& Soeda, H. (1994). Echolocation rates of two harbor poroises (Phocoena phocoena). Marine Mammal Science 10, 401-411.

Akamatsu, T., Matsushita, Y., Hatakeyama, Y. \& Inoue, Y. (1996). Startle response level of Japanese anchovy Engraulis japonicus to underwater pure tone signals. Fisheries Science 62, 648-649.

Aroyan, J. L. (1990a). Numerical simulation of dolphin echolocation beam formation. Master of Science thesis, University of California at Santa Cruz.

Aroyan, J. L. (1990b). Supercomputer modeling of delphinid sonar beam formation. Journal of the Acoustical Society of America 88, S4.

Astrup, J. \& Mfhl, B. (1993). Detection of intense ultrasound by the cod (Gadus morhua). Journal of Experimental Biology 182, 71-80.

Au, W. W. L. (1980). Echolocation signals of the Atlantic bottlenose dolphin (Tursiops truncatus) in open waters. In Animal Sonar Systems (ed. R. G. Busnel and J. F. Fish), pp. 251-282. Plenum Press, New York.

$\mathrm{Au}, \mathrm{W}$. W. L. (1993). The sonar of dolphins. Springer-Verlag, New York.

Au, W. W. L., Moore, P. W. B. \& Pawloski, D. (1986). Echolocation transmitting beam of the Atlantic bottlenose dolphin. The Journal of the Acoustical Society of America 80, 688-691.

Au, W. W. L. \& Penner, R. H. (1981). Target detection in noise by echolocating Atlantic bottlenose dolphins. Journal of the Acoustical Society of America 70, 687-693.

Au, W. W. L. \& Snyder, K. J. (1980). Long-range target detection in open waters by an echolocating Atlantic bottlenose dolphin (Tursiops truncatus). Journal of the Acoustical Society of America 68, 1077-1084.

Au, W. W. L. \& Turl, C. W. (1991). Material composition discrimination of cylinders at different aspect ratios by an echolocating dolphin. Journal of the Acoustical Society of America 89, 2448-2451.

Barclay, R. M. R. (1986). The echolocation calls of hoary (Lasiurus cinereus ) and silverhaired (Lasionycteris noctivagans) bats as adaptations for long-versus shortrange foraging strategies and the consequences for prey selection. Canadian Journal of Zoology 64, 2700-2705. 
Barros, N. B. \& Wells, R. S. (1998). Prey and feeding patterns of resident bottlenose dolphins (Tursiops truncatus) in Sarasota Bay, Florida. Journal of Mammology 79, 1045-1059.

Bel'kovich, V. M., Ivanova, E. E., Yefremenkova, O. V., Kozarovitsky, L. B. \& Kharitonov, S. P. (1991). Searching and hunting behavior in the bottlenose dolphin (Tursiops truncatus) in the Black Sea. In Dolphin Societies: Discoveries and Puzzles (ed. K. Pryor and K. S. Norris), pp. 38-67. University of California Press, Berkeley, CA.

Blaxter, J. H. S., Gray, J. A. B. \& Denton, E. J. (1981). Sound and startle responses in herring shoals. Journal of the Biological Association of the UK 61, 851-869.

Blaxter, J. H. S. \& Hoss, D. E. (1981). Startle response in herring: the effect of sound stimulus frequency, size of fish, and selective interference with the acousticolateralis system. Journal of the Biological Association of the UK 61, 871-879.

Brill, R. L. \& Harder, P. J. (1991). The effects of attenuating returning echolocation signals at the lower jaw of a dolphin (Tursiops turncatus). The Journal of the Acoustical Society of America 89, 2851-2857.

Brill, R. L., Sevenich, M. L., Sullivan, T. J., Sustman, J. D. \& Witt, R. E. (1988). Behavioral evidence for hearing through the lower jaw by an echolocating dolphin (Tursiops truncatus). Marine Mammal Science 4, 223-230.

Britton, A. R. C., Jones, G., Rayner, J. M. V., Booman, A. M. \& Verboom, B. (1997). Flight performance, echolocation, and foraging behavior in pond bats, Myotis dasycneme, (Chiroptera: Vespertilionidae). Journal of Zoology 241, 503-522.

Caldwell, D. K. \& Caldwell, M. C. (1972). The World of the Bottlenose Dolphin. J. B. Lippincott, Philadelphia.

Caldwell, M. C. \& Caldwell, D. K. (1965). Individualized whistle contours in bottlenosed dolphins (Tursiops truncatus). Nature 207, 434-435.

Canfield, J. G. \& Eaton, R. C. (1990). Swimbladder acoustic pressure transduction initiates Mauthner-mediated escape: Nature 347, 760-762.

Cockcroft, V. G. \& Ross, G. J. B. (1990). Age, growth, and reproduction of bottlenose dolphins, Tursiops truncatus, from the east coast of southern Africa. Fishery Bulletin 88, 289-302.

Connor, R. C. \& Smolker, R. A. (1996). 'Pop' goes the dolphin: a vocalization male botttlenose dolphins produce during courtships. Behaviour 133, 643-662. 
Connor, R. C., Smolker, R. A. \& Richards, A. F. (1992). Two levels of alliance formation among male bottlenose dolphins (Tursiops sp.). Proceedings of the National Academy of Sciences USA 89, 987-990.

Craig, W. (1918). Appetites and aversions as constituents of instincts. Biological Bulletin 34, 91-107.

Cranford, T. W. (1988). Comparative morphology of the odontocete nose: anatomy of a sonar signal generator. In Animal Sonar: Processes and Performance (ed. P. E. Nachtigall and P. W. B. Moore), pp. 67-77. Plenum Press, New York.

Dear, S. P. \& Suga, N. (1995). Delay-tuned neurons in the midbrain of the big brown bat. Journal of Neurophysiology 73, 1084-1100.

dos Santos, M. E., Caporin, G., Moreira, H. O., Ferreira, A. J. \& Coelho, J. L. B. (1990). Acoustic behavior in a local population of bottlenose dolphins. In Sensory Abilities of Cetaceans (ed. J. Thomas and R. Kastelein), pp. 585-598. Plenum Press, New York.

Duffield, D. \& Wells, R. (1991). The combined application of chromosome, protein and molecular data for the investigation of social unit structure and dynamics in Tursiops truncatus. Report of the International Whaling Commission 13, 155-169.

Dunning, D. J., Ross, Q. E., Geoghegan, P., Reichle, J. J., Menezes, J. K. \& Watson, J. K. (1992). Alewives avoid high-frequency sound. North American Journal of Fisheries Management 12, 407-416.

Eaton, R. C., Bombardieri, R. A. \& Meyer, D. L. (1977). The mauthner-initiated startle response in teleost fish. Journal of Experimental Biology 66, 65-81.

Emde, G. v. d. \& Schnitzler, H.-U. (1990). Classification of insects by echolocating greater horseshoe bats. Journal of Comparative Physiology A 19, 121-136.

Evans, R. M. (1982). Efficient use of food patches at different distances from a breeding colony in black-billed gulls. Behaviour 79, 28-38.

Evans, W. E. \& Powell, B. A. (1967). Discrimination of different metallic plates by an echolocating delphinid. In Animal Sonar Systems: Biology and Bionics (ed. R. G. Busnel), pp. 363-382. Laboratoire de Physiologie Acoustique, Jouy-en-Josas, France.

Faure, P. A. \& Barclay, R. M. R. (1994). Substrate-gleaning versus aerial-hawking: Plasticity in the foraging and echolocation behaviour of the long-eared bat, Myotis evotis. Journal of Comparative Physiology, A 174, 651-660. 
Fay, R. R. (1988). Hearing in Vertebrates: a Psychophysics Databook. Hill-Fay, Winnetka, IL.

Fiedler, J. (1979). Prey catching with and without echolocation in the Indian false vampire (Megaderma lyra). Behavioral Ecology 6, 155-160.

Fish, M. P. \& Mowbray, W. H. (1970). Sounds of western north Atlantic fishes: a reference file of biological underwater sounds. Johns Hopkins Press, Baltimore.

Focardi, S., Marcellini, P. \& Montanaro, P. (1996). Do ungulates exhibit a food density threshold? A field study of optimal foraging and movement patterns. Journal of Animal Ecology 65, 606-620.

Fullard, J. H., Fenton, M. B. \& Simmons, J. A. (1979). Jamming bat echolocation: the clicks of arctiid moths. Canadian Journal of Zoology 57, 647-649.

Fullard, J. H., Simmons, J. A. \& Saillant, P. A. (1994). Jamming bat echolocation: the dogbane tiger moth Cycnia tenera times its clicks to the terminal attack calls of the big brown bat Eptesicus fuscus. Journal of Experimental Biology 194, 284298.

Goss-Custard, J. D., Caldow, R. W. G., Clarke, R. T. \& West, A. D. (1995). Deriving population parameters from individual variations in foraging behavior. II. Model tests and population parameters. Journal of Animal Ecology 64, 277-289.

Goss-Custard, J. D. \& Le V dit Durell, S. E. A. (1983). Individual and age differences in the feeding ecology of oystercatchers Haematopus ostralegus wintering on the Exe Estuary, Devon. Ibis 125, 155-171.

Goss-Custard, J. D., Le V dit Durell, S. E. A. \& Ens, B. J. (1982). Individual differences in aggressiveness and food stealing among wintering oystercatchers, Haematopus ostralegus. Animal Behavior 30, 917-928.

Goss-Custard, J. D. \& Sutherland, W. J. (1984). Feeding specializations in oystercatchers, Haepmatopus ostralegus. Animal Behavior 32, 299-301.

Griffin, D. R. (1958). Listening in the Dark. Yale University Press, New Haven.

Griffin, D. R., Webster, F. A. \& Michael, C. R. (1960). The echolocation of flying insects by bats. Animal Behavior 8, 141-154.

Grinnel, A. D. (1995). Hearing in bats: an overview. In Hearing by Bats (ed. A. N. Popper and R. R. Fay), pp. 1-36. Springer, New York. 
Gunter, G. (1942). Contributions to the natural history of the bottlenose dolphins, Tursiops truncatus (Montague), on the Texas coast, with particular reference to food habits. Journal of Mammology 23, 267-276.

Hamilton, P. V. \& Nishimoto, R. T. (1977). Dolphin predation on mullet. Florida Scientist 40, 251-252.

Hammer, C. E., Jr. \& Au, W. W. L. (1980). Porpoise echo-recognition: an analysis of controlling target characteristics. Journal of the Acoustical Society of America 68 , 1285-1293.

Haskell, P. T. \& Belton, P. (1956). Arctiidae with tympanic organs capable of detecting bat echolocation. Nature 117, 139.

Hemmi, J. M. \& Menzel, C. R. (1995). Foraging strategies of long-tailed macaques, Macaca fascicularis: directional extrapolation. Animal Behaviour 49, 457-463.

Herman, L. M. \& Tavolga, W. N. (1980). The communication system of cetaceans. In Cetacean Behavior (ed. L. M. Herman), pp. 149-209. John Wiley and Sons, New York.

Hoelzel, A. R., Dorsey, E. M. \& Stern, S. J. (1989). The foraging specializations of individual minke whales. Animal Behavior 38, 786-794.

Hoese, H. D. (1971). Dolphins feeding out of water in a salt marsh. Journal of Mammology 52, 222-223.

Hohn, A. A., Scott, M. D., Wells, R. S., Sweeney, J. C. \& Irvine, A. B. (1989). Growth layers in teeth from known-age, free-ranging bottlenose dolphins. Marine Mammal Science 5, 315-342.

Irvine, A. B., Scott, M. D., Wells, R. S. \& Kaufmann, J. H. (1981). Movements and activities of the Atlantic bottlenose dolphin, Tursiops truncatus, near Sarasota, Florida. Fishery Bulletin 79, 671-688.

Irvine, B. \& Wells, R. S. (1972). Results of attempts to tag Atlantic bottlenose dolphins, Tursiops truncatus. Cetology 13, 1-5.

Jones, G. (1990). Prey selection by the greater horseshoe bat (Rhinolophus ferrumequinum): optimal foraging by echolocation? Journal of Animal Ecology $\mathbf{5 9 , 5 8 7 - 6 0 2 . ~}$

Jones, G. (1995). Flight performance, echolocation, and foraging behavior in noctule bats, Nyctalus noctula. Journal of Zoology 237, 303-312. 
Jones, G., Morton, M., Hughes, P. M. \& Budden, R. M. (1993). Echolocation, flight morphology and foraging strategies of some West African hipposiderid bats. Journal of Zoology 230, 385-400.

Kalko, E. K. V. (1995). Insect pursuit, prey capture and echolocation in pipistrelle bats (Microchiroptera). Animal Behaviour 50, 861-880.

Kalko, E. K. V., Schnitzler, H.-U. \& Grinnel, A. D. (1998). Echolocation and foraging behavior of the lesser bulldog bat, Noctilio albiventris: preadaptations for pisciviory? Behavioral Ecology and Sociobiology 42, 305-319.

Kellogg, W. N. (1961). Porpoises and sonar. University of Chicago Press, Chicago.

Kemp, R. J. (1949). Report on the stomach analysis - Delphininae, pp. 111-112, 126-127. Annual Report of the Marine Laboratory of the Texas Game, Fish, and Oyster Community.

Kleinenberg, S. E. (1938). Nekotorye dannye o pitanii Tursiops tursio Fabr. v Chernom more (Some data on feeding of Turiops tursio Fabr. in the Black Sea). Bulletin Soc. Nat. Moscow 47, 406-413.

Kober, R. \& Schnitzler, H.-U. (1990). Information in sonar echoes of fluttering insects available for echolocating bats. The Journal of the Acoustical Society of America 87, 882-896.

Le V. dit Durell, S. E. A. \& Goss-Custard, J. D. (1984). Prey selection within a size-class of mussels, Mytilus edulis, by oystercatchers, Haematopus ostralegus. Animal Behavior 32, 1197-1203.

Leatherwood, S. (1975). Some observations of feeding behavior of bottle-nosed dolphins (Tursiops truncatus) in the northern Gulf of Mexico and (Tursiops cf $T$. gilli) off southern California, Baja California, and Nayarit, Mexico. Marine Fisheries Review 37, 10-16.

Leatherwood, S., Deerman, M. W. \& Petter, C. W. (1978). Food and reproductive status of nine Tursiops truncatus from the northeastern United States coast. Cetology 28, $1-6$.

Lopez, J. C. \& Lopez, D. (1985). Killer whales (Orcinus orca) of Patagonia, and their behavior of intentional stranding while hunting near shore. Journal of Mammalogy 66, 181-183.

Mann, D. A., Lu, Z. \& Popper, A. N. (1997). A clupeid fish can detect ultrasound. Nature 389,341 . 
Mead, J. G. \& Potter, C. W. (1990). Natural history of the bottlenose dolphins along the central Atlantic coast of the United States. In The Bottlenose Dolphin (ed. S. Leatherwood and R. R. Reeves), pp. 165-195. Academic Press, San Diego.

Miller, L. A. (1983). How insects detect and avoid bats. In Neuroethology and Behavioral Physiology (ed. F. Huber and H. Markl), pp. 251-266. SpringerVerlag, Berlin, Heidelberg.

Miller, L. A. (1991). Arctiid moth clicks can degrade the accuracy of range difference discrimination in echolocating big brown bats, Eptesicus fuscus. Journal of Comparative Physiology A 168, 571-579.

Miller, L. A., Futtrup, V. \& Dunning, D. C. (1996). Prey capture by the bat, Pipistrellus pipistrellus, in the presence of extraneous sounds. Journal of Comparative Physiology A 120, 165-179.

Miller, L. A., Pristed, J., Mfhl, B. \& Surlykke, A. (1995). The click-sounds of narwhals (Monodon monoceros) in Inglefield Bay, Northwest Greenland. Marine Mammal Science 11, 491-502.

Moss, C. F. \& Zagaeski, M. (1994). Acoustic information available to bats using frequency-modulated sounds for the perception of insect prey. The Journal of the Acoustical Society of America 95, 2745-2756.

Murchison, A. E. (1980). Maximum detection range and range resolution in an echolocating bottlenose porpoise (Tursiops truncatus). In Animal Sonar Systems (ed. R. G. Busnel and J. F. Fish), pp. 43-70. Plenum Press, New York.

Neuweiler, G. (1983). Echolocation and adaptivity to ecological constraints. In Neuroethology and Behavioral Physiology (ed. F. Huber and H. Markl), pp. 280302. Springer-Verlag, Berlin.

Norris, K. S. \& Harvey, G. W. (1974). Sound transmission in the porpoise head. Journal of the Acoustical Society of America 56, 659-664.

Norris, K. S. \& Mfhl, B. (1983). Can odontocetes debilitate prey with sound? The American Naturalist 122, 85-104.

Norris, K. S., Prescott, J. H., Asa-Dorian, P. V. \& Perkins, P. (1961). An experimental demonstration of echolocation behavior in the porpoise, Tursiops truncatus (Montagu). Biological Bulletin 120, 163.

Nowacek, D. P., Lange, W. N., Wells, R. S. \& Tyack, P. L. (1995). A new method for studying cetacean behavior and acoustics: overhead video combined with underwater audio. In Eleventh biennial conference on the biology of marine mammals, Orlando, FL. 
Petricig, R. (1995). Bottlenose dolphins (Tursiops truncatus) in Bull Creek, South Carolina. Ph.D. thesis, University of Rhode Island.

Pilleri, G. (1990). Adaptation to water and the evolution of echolocation in the cetacea. Ethology, Ecology, and Evolution 2, 135-163.

Popper, A. N. (1980). Sound emission and detection by delphinids. In Cetacean Behavior (ed. L. M. Herman), pp. 1-53. John Wiley and Sons, New York.

Popper, A. N. \& Fay, R. R. (1988). Comparative studies of hearing in vertebrates. Springer-Verlag, New York.

Popper, A. N. \& Fay, R. R. (1995). Hearing by Bats. Springer, New York.

Rapaport, L. G. (1998). Optimal foraging theory predicts effects of environmental enrichment in a group of adult golden lion tamarins. Zoo Biology 17, 231-244.

Read, A., Wells, R., Scott, M. \& Hohn, A. (1993). Patterns of growth in wild bottlenose dolphins, Tursiops truncatus. Journal of Zoology 231, 107-123.

Reidenberg, J. \& Laitmann, J. (1988). Existence of vocal folds in the larynx of odontoceti (toothed whales). Anatomical Record 221, 886-891.

Roeder, K. D. (1962). The behaviour of free flying moths in the presence of artificial ultrasonic pulses. Animal Behavior 10, 300-304.

Roeder, K. D. (1967). Turning tendency of moths exposed to ultrasound while in stationary flight. Journal of Insect Physiology 13, 873-888.

Roeder, K. D. \& Treat, A. E. (1957). Ultrasonic reception by the tympanic organ of noctuid moths. Journal of Experimental Zoology 134, 127-158.

Rogers, P. H., Popper, A. N., Hastings, M. C. \& Saidel, W. M. (1988). Processing of acoustic signals in the auditory system of bony fish. The Journal of the Acoustical Society of America 83, 338-348.

Rogers, T. \& Bryden, M. M. (1995). Predation of adelie penguins (Pygoscelis adeliae) by leopard seals (Hydrurga leptonyx) in Prydz Bay, Antarctica. Canadian Journal of Zoology 73, 1001-1004.

Ryan, M. J. \& Tuttle, M. D. (1983). The ability of the frog-eating bat to discriminate among novel and potentially poisonous frog species using acoustic cues. Animal Behavior 31, 827-833. 
Ryan, M. J., Tuttle, M. D. \& Barclay, R. M. R. (1983). Behavioral responses of the frogeating bat, Trachops cirrhosus, to sonic frequencies. Journal of Comparative Physiology A 150, 413-418.

Rydell, J. (1993). Variation in the sonar of an aerial-hawking bat (Eptesicus nilssonii). Ethology 93, 275-284.

Sayigh, L. S., Tyack, P. L. \& Wells, R. S. (1993). Recording underwater sounds of freeranging dolphins while underway in a small boat. Marine Mammal Science $\mathbf{9}$, 209-213.

Sayigh, L. S., Tyack, P. L., Wells, R. S. \& Scott, M. D. (1990). Signature whistles of free-ranging bottlenose dolphins, Tursiops truncatus: stability and motheroffspring comparisons. Behavioral Ecology and Sociobiology 26, 247-260.

Sayigh, L. S., Tyack, P. L., Wells, R. S., Scott, M. D. \& Irvine, A. B. (1995). Sex differences in signature whistle production of free-ranging bottlenose dolphins, Tursiops truncatus. Behavioral Ecology and Sociobiology 36, 171-177.

Schevill, W. E. \& Lawrence, B. (1953a). Auditory response of a bottle-nosed porpoise, Tursiops truncatus, to frequencies above $100 \mathrm{kc}$. Journal of Experimental Zoology 124, 147-165.

Schevill, W. E. \& Lawrence, B. (1953b). High-frequency auditory response of a bottlenosed dolphin, Tursiops truncatus (Montagu). Journal of the Acoustical Society of America 24, 1016-1017.

Schmid-Hempel, P., Kacelnik, A. \& Houston, A. I. (1985). Honeybees maximize efficiency by not filling their crop. Behavioral Ecology and Sociobiology 17, 6166.

Schnitzler, H.-U., Kalko, E. K. V., Kaipf, I. \& Grinnel, A. D. (1994). Fishing and echolocation behavior of the greater bulldog bat, Noctilio leporinus, in the field. Behavioral Ecology and Sociobiology 35, 327-345.

Scott, M. D., Wells, R. S. \& Irvine, A. B. (1990). A long-term study of bottlenose dolphins on the west coast of Florida. In The Bottlenose Dolphin (ed. S. Leatherwood and R. R. Reeves), pp. 235-244. Academic Press, San Diego.

Shane, S. H. (1990). Behavior and ecology of the bottlenose dolphin at Sanibel Island, Florida. In The Bottlenose Dolphin (ed. S. Leatherwood and R. R. Reeves), pp. 245-265. Academic Press, San Diego.

Shane, S. H., Wells, R. S. \& Würsig, B. (1986). Ecology, behavior, and social organization of the bottlenose dolphin: a review. Marine Mammal Science 2, 3463. 
Simmons, J. A., Fenton, M. B. \& O'Farrell, M. J. (1979). Echolocation and pursuit of prey by bats. Science 203, 16-21.

Simmons, J. A., Moss, C. F. \& Ferragamo, M. (1990). Convergence of temporal and spectral information into acoustic images of complex sonar targets perceived by the echolocating bat, Eptesicus fuscus. Journal of Comparative Physiology A 166, 449-470.

Stephens, D. W. \& Krebs, J. R. (1986). Foraging Theory. Princeton University Press, Princeton.

Swennen, C., De Bruijn, L. L. M., Duiven, P., Leopold, M. F. \& Marteijn, E. C. L. (1983). Differences in bill form of the oystercatcher, Haematopus ostralegus; a dynamic adaptation to specific foraging techniques. Netherlands Journal of Sea Research 17, 57-83.

Tayler, C. K. \& Saayman, G. S. (1972). The social organisation and behavior of dolphins (Tursiops adunctus) and baboons (Papio ursinus): Some comparisons and assessments. Annals of the Cape Provincial Museum 9, 11-49.

Tinbergen, N. (1951). The Study of Instinct. Oxford University Press, Oxford.

Tsalkin, V. I. (1940). Nekotorye nablyudeniya nad biologiei delfinov Azobskovo i Chernovo morei (Some observations of the biology of the Azov and Black Sea dolphins). Bulletin Soc. Nat. Moscow 49, 61-70.

Turl, C. W. \& Penner, R. H. (1989). Differences in echolocation click patterns of the beluga (Delphinapterus leucas) and the bottlenose dolphin (Tursiops truncatus). Journal of the Acoustical Society of America 86, 497-502.

Tuttle, M. D. \& Ryan, M. J. (1981). Bat predation and the evolution of frog vocalizations in the neotropics. Science 214, 677-678.

Tuttle, M. D., Ryan, M. J. \& Belwood, J. J. (1985). Acoustical resource partitioning by two species of phyllostomid bats (Trachops cirrhosus and Tonatia sylvicola). Animal Behavior 33, 1369-1370.

Van Waerebeek, K., Reyes, J. C., Read, A. J. \& McKinnon, J. S. (1990). Preliminary observations of bottlenose dolphins from the Pacific coast of South America. In The Bottlenose Dolphin (ed. S. Leatherwood and R. R. Reeves), pp. 143-154. Academic Press, San Diego.

Verfuss, U. K. \& Schnitzler, H. U. (1995). Hunting and echolocation behavior of a captive Amazon river dolphin (Inia geoffrensis). In Eleventh Biennial Conference on the Biology of Marine Mammals, 14-18 December, Orlando, FL. 
Waples, D. M., Wells, R. S., Costa, D. P. \& Worthy, G. A. J. (1995). Seasonal changes in activity and habitat use by bottlenose dolphins (Tursiops truncatus) in Sarasota Bay, FL. In Eleventh Biennial Conference on the Biology of Marine Mammals, Orlando, FL.

Weinrich, M. T., Schilling, M. R. \& Belt, C. R. (1992). Evidence for acquisition of a novel feeding behaviour: lobtail feeding in humpback whales, Megaptera novaeangliae. Animal Behavior 44, 1059-1072.

Wells, R. S. (1991). The role of long-term study in understanding the social structure of a bottlenose dolphin community. In Dolphin Societies: Discoveries and Puzzles (ed. K. Pryor and K. S. Norris), pp. 199-225. University of California Press, Berkeley.

Wells, R. S., Irvine, A. B. \& Scott, M. D. (1980). The social ecology of inshore odontocetes. In Cetacean Behavior: Mechanisms and Functions (ed. L. M. Herman), pp. 263-317. John Wiley \& Sons, New York.

Wells, R. S. \& Scott, M. D. (1999). Bottlenose dolphin Tursiops truncatus (Montagu, 1821). In Handbook of Marine Mammals, the Second Book of Dolphins and Porpoises, vol. 6 (ed. S. H. Ridgway and R. Harrison), pp. 137-182. Academic Press, San Diego.

Wells, R. S., Scott, M. D. \& Irvine, A. B. (1987). The social structure of free-ranging bottlenose dolphins. In Current Mammalogy, vol. 1 (ed. H. H. Genoways), pp. 247-305. Plenum Press, New York.

Yamagiwa, J. \& Mwanza, N. (1994). Day-journey length and daily diet of solitary male gorillas in lowland and highland habitats. International Journal of Primatology 15, 207-224.

Zhantiyev, R. D., Lapshin, D. N. \& Fedorova, M. V. (1993). Emission and perception of ultrasound in moths (lepidotera, noctuidae). Entomological Review 72, 97-105. 


\section{General Materials \& Methods}

\subsection{Introduction}

The research questions addressed by this study require integrated research methodologies and a field site where these methods can be effectively and safely applied. An ideal setting for this type of study is the Sarasota Dolphin Research Program (SDRP) ongoing study of the resident bottlenose dolphins in Sarasota Bay, FL. The background knowledge of the animals combined with the capture-release opportunities (see Section 1.1) provide a unique situation in which to study known wild animals.

The primary goal of both behavioral and acoustic data collection was to record specific foraging behaviors and the corresponding acoustic activity. Behavioral data were collected using a combination of observational methods and protocols. Continuous and instantaneous sampling were used during focal-animal follows (Altmann, 1974). The main goal of the continuous sampling was to record specific behaviors from sequential stages of foraging: search, pursuit, capture, and handling, some of which are brief and/or subtle. Recording specific, brief, and subtle behaviors can be challenging for any animal but especially for one that spends the vast majority of its time underwater. To address both the sub-surface nature and the transience of these behaviors an integrated observation platform (IOP) combining overhead video with underwater acoustic recordings was used for continuous sampling (Appendix 1). Briefly, the system consists of a video camera suspended from a helium-filled airship that is tethered to the observation vessel (OV). The video image travels down the combined electrical/strength tether to a video recorder on the OV, then to a video monitor that is being watched by the camera operator. The operator has full control of the camera (pan-tilt, zoom, focus, iris), and adjusts it to maintain the best image (e.g. focused, full frame image of the dolphin). An overhead vantage point affords greater visibility into the water column (see Appendix 1), and a video record permits a detailed review of even brief, subtle behaviors. While 
observing a focal animal from the overhead video platform, instantaneous samples were collected from the observation vessel.

Acoustic data were collected by two methods. As part of the IOP two hydrophones were towed through the water using a system developed by Sayigh et al. (1993). These hydrophones were connected through a high-pass filter to the audio input of the overhead video recorder. The second method used to collect acoustic data was a recording tag (Nowacek et al., 1998) attached to temporarily restrained animals which were, after release, followed according to the focal-animal protocol described below.

\subsection{Behavioral Data Collection}

\subsubsection{Focal-Animal Continuous Sampling}

Continuous behavioral sampling during focal-animal follows (Altmann, 1974) was conducted exclusively from video tapes recorded from the IOP. Video tapes, including acoustic activity, were reviewed and scored in the laboratory; this process is described fully in section 4.2. Each day the study area (Figure 1) was searched until appropriate animals were located. The sample of individuals studied represents both subadult and adult animals. Dependent animals, i.e. those always sighted with their mothers, were excluded from the study for two reasons. Their feeding rate is presumably lower than independent animals as they may still be nursing, and their foraging patterns are still developing (Caro \& Hauser, 1992; Haenel, 1986; Lopez \& Lopez, 1985) which could give a biased view of specific behaviors observed. Once animals were located, basic data were collected according to the Sarasota Dolphin Research Program (SDRP) protocol including: initial heading; animal identifications and/or photographs; numbers of adult animals, calves, and young of the year (YOYs); location (GPS and code); environmental conditions; and general activity (Urian \& Wells, 1996). If one of the animals present was considered to be appropriate, i.e. not a dependent calf nor a highly repeated subject (sampled $\geq 5$ hours), a focal follow was begun. For continuous sampling the goal was to 
maintain the subject in the video field of view. If the subject was lost from view the boat crew would assist the 'camera driver' in relocating the animal. The periods of time during which the animal was not within the video field will be discussed in section 4.2 . While taping the subject, the 'camera-driver' would maintain the best possible image, which includes tightest possible zoom (often dictated by animal's speed), centered image, focus, and most favorable iris setting. The format for the majority of the video footage was Hi-8, but early segments were recorded on BetaSP and later segments on digital video tape.

\subsubsection{Focal-Animal Instantaneous Sampling}

Instantaneous sampling (Altmann, 1974) was utilized to record a variety of data including group spread, location, habitat type, and environmental conditions. Altmann (1974) describes instantaneous sampling as an effective method of estimating the amount of time individuals devote to specific activities. The instantaneous sampling protocol was designed, therefore, to measure behavioral state parameters related to foraging.

A change in group spread has been associated with foraging in inshore bottlenose dolphins (Irvine et al., 1981), so to quantify this the focal animal's distance to nearest neighbor was recorded in 1996, and in 1997 additional measures of overall group spread were added. Next, the habitat type and depth of water were recorded to investigate in greater detail the general habitat use reported by Waples (1995) and because the distribution of their prey items is affected by these parameters (Savino \& Stein, 1989; Sogard, Powell \& Holmquist, 1989). The 1996 data indicated that in some instances the dolphins travel directly between prey-rich areas, e.g. between shallow seagrass patches. In response the 1997 data collection protocol included GPS location so the animals' general movements could be tracked. Also added in 1997 was a measurement of water clarity (secchi disc), because data collected in 1996 suggested that dolphins may echolocate less frequently in clearer water. Finally, measurements of sea state, cloud 
cover, current, and tide were recorded to investigate the influence of these factors on foraging activity.

\subsection{Acoustic Data Collection}

\subsubsection{Towed Acoustic System}

As part of the IOP two hydrophones (HTI-96) were towed through the water using a system virtually identical to Sayigh et al. (1993). These hydrophones were highpass filtered (Allen Avionics F4188-4PO; $\mathrm{F}_{-3 \mathrm{~dB}}=4 \mathrm{kHz}, 6-$ pole) and then input into the audio channels of the video recorder (for $\mathrm{Hi}-8$ : frequency response is flat to approximately $30 \mathrm{kHz}$ ). Some acoustic signals of interest have low frequency components so when attempting to record these the filter was taken offline. This audio recording system yielded excellent quality recordings of the anticipated clicks (Figure 5.1a) and whistles (Figure 5.1b), and also recorded unexpected sounds such as 'pops' (Figure 5.1c). In regards to clicks the recordings from this system could not be used to identify the animal which made the sounds, but since many of the follows were conducted on single animals the rate of click train production could still be estimated. The results of these echolocation recordings are likely negatively biased because the high degree of directionality of echolocation clicks (Au, Floyd \& Haun, 1978; Au, Moore \& Pawloski, 1986) precludes the recording of every click even for single-animal follows.

\subsubsection{Acoustic Data Logger}

To record echolocation click events (i.e. not full bandwidth recordings) known to be from a specific individual, an acoustic data logger was developed (see Chapter 3 and Appendix 2). The logger was attached to temporarily restrained dolphins during the capture-release project (see Chapter 1). The animals were subsequently released and followed with the IOP according to the standard focal-animal follow protocol. 
The data logger was deployed during the 1997 and 1998 capture-release projects. A summary of the data logger equipment and operation is provided here; for a full description see (Nowacek et al., 1998). Both the 1997 and 1998 versions consisted of suction cup hydrophones connected through a water-tight housing to an audio recorder (Sony NT-2; Fs=32 kHz), and the housing was attached to the dolphin's fin with a noninvasive Trac $\mathrm{Pac}^{\circledR}$. A VHF radio transmitter was also incorporated into the Trac $\mathrm{Pac}{ }^{(1)}$ to facilitate following the animal and to recover the pack after its release. The 1997 version had two suction cup hydrophones: an 'echolocation' sensor which was placed on the dorsal mid-line approximately $20 \mathrm{~cm}$ posterior of the blow hole, and an 'ambient' sensor located on the body flank at the base of the dorsal fin. In 1998 a hydrophone was attached only at the 'ambient' location as experiments with captive animals at the Dolphin Research Center (DRC) showed that each echolocation click event could be recorded at that location (see Section 3.3.1). The only other unique feature of the 1997 logger was the use of a simple demodulator in one deployment. The theory of the demodulator was to utilize the band of highest click energy, i.e. $70-90 \mathrm{kHz}$, thereby assuring successful recording of each click. Two results alleviated the need for the demodulator: 1) the animals' low click rate; and 2) the ability to record every click event at the 'ambient' sensor location.

In 1998 a number of features were added to improve the data logger's sampling capability with the goal being to sample the animal's acoustic activity while recording indicators of its behavior. The attitude tag (ATAG) incorporated an electrostatic pitchroll sensor, a digital 3-dimensional compass, and a sound pressure level sensor all of which were sampled at either 1 or $2 \mathrm{~Hz}$. The data were recorded on a $1 \mathrm{MB}$ non-volatile serial flash memory chip with memory capacity of 36 hours at $1 \mathrm{~Hz}$ sampling, 18 hours at $2 \mathrm{~Hz}$. The ATAG also controlled the audio recorder allowing the 2 stereo-hours of tape to be recorded according to a pre-set schedule. 


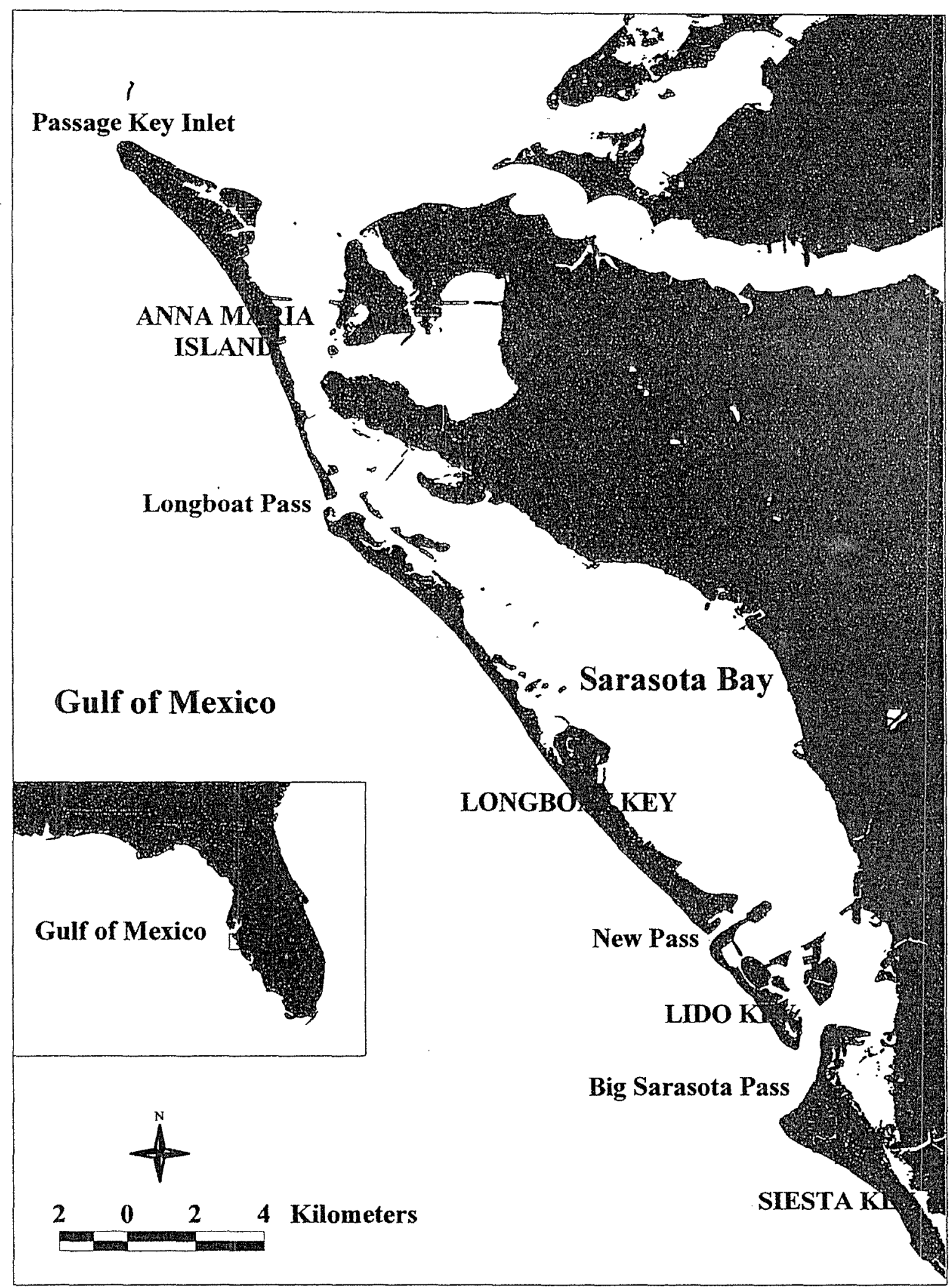

Figure 1. Map of study area. 


\subsection{Literature Cited}

Altmann, J. (1974). Observational study of behavior: sampling methods. Behaviour 49: 227-267.

Au, W. W. L., Floyd, R. W. \& Haun, J. E. (1978). Propagation of Atlantic bottlenose dolphin echolocation signals. The Journal of the Acoustical Society of America 64: 411-422.

Au, W. W. L., Moore, P. W. B. \& Pawloski, D. (1986). Echolocation transmitting beam of the Atlantic bottlenose dolphin. The Journal of the Acoustical Society of America 80: 688-691.

Caro, T. M. \& Hauser, M. D. (1992). Is there teaching in nonhuman animals? Quarterly Review of Biology 67: 151-174.

Haenel, N. J. (1986). General notes on the behavioral ontogeny of Puget Sound killer whales and the occurrence of allomaternal behavior. In Behavioral Biology of Killer Whales (ed. B. C. Kirkevold and J. S. Lockard), pp. 285-300. Alan R. Liss, Inc., New York.

Irvine, A. B., Scott, M. D., Wells, R. S. \& Kaufmann, J. H. (1981). Movements and activities of the Atlantic bottlenose dolphin, Tursiops truncatus, near Sarasota, Florida. Fishery Bulletin 79: 671-688.

Lopez, J. C. \& Lopez, D. (1985). Killer whales (Orcinus orca) of Patagonia, and their behavior of intentional stranding while hunting near shore. Journal of Mammalogy 66: 181-183.

Nowacek, D. P., Tyack, P. L., Wells, R. S. \& Johnson, M. P. (1998). An onboard acoustic data logger to record biosonar of free-ranging bottlenose dolphins. Journal of the Acoustical Society of America 103: 1409-1410.

Savino, J. F. \& Stein, R. A. (1989). Behavior of fish predators and their prey: habitat choice between open water and dense vegetation. Environmental Biology of Fishes 24: 287-293.

Sayigh, L. S., Tyack, P. L. \& Wells, R. S. (1993). Recording underwater sounds of freeranging dolphins while underway in a small boat. Marine Mammal Science 9: 209-213. 
Sogard, S. M., Powell, G. V. N. \& Holmquist, J. G. (1989). Utilization by fishes of shallow, seagrass-covered banks in Florida Bay: 1. species composition and spatial heterogeneity. Environmental Biology of Fishes 24: 53-65.

Urian, K. W. \& Wells, R. S. (1996). Bottlenose dolphin photo-identification workshop, pp. 92. National Marine Fisheries Service, Southeast Fisheries Science Center, Charleston, SC.

Waples, D. M., Wells, R. S., Costa, D. P. \& Worthy, G. A. J. (1995). Seasonal changes in activity and habitat use by bottlenose dolphins (Tursiops truncatus) in Sarasota Bay, FL. In Eleventh Biennial Conference on the Biology of Marine Mammals, Orlando, FL. 


\section{Chapter 3. Acoustic Activity of Free-ranging Bottlenose Dolphins}

\subsection{Introduction}

Animals produce many different types of signals for a variety of purposes, and the behavioral ecologist works to elucidate the ecological function of the signals. Animals use all of the primary sensory and perceptual pathways for signaling: vision, hearing, touch, and taste or smell (i.e. chemical). The uses range, in general terms, from intraspecific communication to individually important needs such as navigation and food finding. Behavioral ecologists require at least three critical pieces of data to understand the details of a signal's function: 1) to record it in a biologically relevant setting, 2) to know the individual that produced it, and 3 ) to record the preceding and ensuing behavior of the signaler and other potential respondents within range of the signal. A well studied signaling system can illustrate the depth of understanding possible with such data.

The structure and functions of vervet monkey, Cercopithecus aethiops, acoustic signals are well understood because these critical data were collected early in the study of this signaling system. Struhsaker (1967) carefully cataloged 36 distinct sounds and their functions based on his recordings and observations of wild vervet monkeys in their natural habitat. He knew which animal was signaling through the use of a parabolic microphone and because characteristic mouth and lip movements accompanied each sound (Struhsaker, 1967), and his thousands of hours of observation provided the behavioral context from which he deduced the function of the sounds. The results from, and continued use of, these techniques have revealed very detailed information about this signaling system. For example, Cheney and Seyfarth (1980), used a protocol similar to Struhsaker's to evaluate the selective forces affecting vervet monkey alarm calls. With the extensive baseline knowledge these and other studies produced, an additional technique could be utilized to address even more specific questions. Acoustic playback experiments (i.e. recreating signal stimuli) can be a powerful means of testing hypotheses (Cheney \& Seyfarth, 1980; Cheney \& Seyfarth, 1982; Horn, 1992; Searcy, 1992), but 
such experiments must be conducted with caution as the results can be confounded by many factors (Gerhardt, 1992; McGregor, 1992). Cheney and Seyfarth skillfully used playback experiments to demonstrate that vervet monkeys can recognize individuals (1982) including mother-infant recognition (1980). They also showed that vervets can assess the meaning and reliability of signals from particular individuals (Cheney \& Seyfarth, 1988).

Identifying which individual makes a signal is therefore an essential.step in gaining an understanding of the function of that signal for the signaler and others. Confidently assigning a sound to a particular individual has been a long-standing difficulty in the study of wild marine mammals, especially cetaceans. For free-ranging cetaceans there are two methods a researcher can employ to reduce the sound source ambiguity to acceptable levels. An array of hydrophones mounted on the sea floor, suspended from buoys, or towed through the water can provide adequate data for localizing which animal produced a sound (Clark, Ellison \& Beeman, 1986; Clark \& Johnson, 1984; Miller \& Tyack, 1998; Watkins \& Schevill, 1972). The other method is to have a sensor and acoustic recorder attached to the individual (i.e. data logger). This method has been used successfully with pinnipeds (Fletcher et al., 1996), but not freeranging cetaceans. Recording odontocete biosonar presents a particularly difficult technical issue due to the high frequencies and narrow transmit beam pattern of these sounds (Au, 1993). Given these constraints, the present work sought to record the occurrence of every biosonar pulse, not the full spectrum of the signals.

Being able to confidently assign a sound to a particular individual in its natural setting provides two of the three pieces of information identified as critical for understanding the ecology of a particular signal. The present chapter demonstrates the successful application of a data logger to free-ranging bottlenose dolphins. Traditionally three different types of sounds have been attributed to bottlenose dolphins: whistles, burst-pulse sounds, and echolocation clicks (Popper, 1980). Whistles are frequency 
modulated tonal sounds with fundamental frequencies ranging from $2-20 \mathrm{kHz}$ (Caldwell \& Caldwell, 1965). Burst-pulse sounds encompass a wide variety of sounds that are highly variable in structure and length (Caldwell \& Caldwell, 1967; Wood, 1953). Echolocation clicks are short $(50-150 \mu \mathrm{sec})$ broadband pulses with center frequencies between $65-120 \mathrm{kHz}$ and $-3 \mathrm{~dB}$ bandwidth of approximately $40 \mathrm{kHz}(\mathrm{Au}, 1993)$. The spectral content of the clicks does vary, although $\mathrm{Au}$ (1993) asserts that there is no evidence that bottlenose dolphins adapt the spectrum for specific tasks. Amundin (1991), however, has found spectral adaptation in harbor porpoises, Phocoena phocoena. For the following reasons the data logger was designed to record simply the occurrence of every echolocation click (i.e. the timing of each click relative to specific tasks and to other clicks) not the full bandwidth. 1) Even for captive dolphins there is debate as to whether the animals always emit clicks with inter-click intervals (ICI: the time between the onset of two successive clicks) greater than the two way travel time to the target (Ivanov \& Popov, 1979; Norris et al., 1961; Turl \& Penner, 1989). 2) Knowledge of the timing and use patterns of bat echolocation has provided significant insight into the ecology of the bat echolocation system (Kick \& Simmons, 1984; Schnitzler \& Henson, 1980; Schnitzler et al., 1994). 3) The high frequencies, narrow transmit beam pattern, limited access to only the near field, and broad signal band of dolphin echolocation (Au, 1993) make these signals difficult to sample completely. All other sounds dolphins are known to produce were well within the recording capabilities of the data logger (Nowacek et al., 1998; Popper, 1980).

\subsection{Materials and Methods}

\subsubsection{Test for Sensor Location}

Given the objective of reliably recording every click event, an appropriate location for the suction cup hydrophone had to be determined. Three potential sensor locations were identified with the goal of finding a compromise between minimizing effects on the animal, minimizing flow disruption and noise, the practicality of 
attachment, and the ability to record the occurrence of each click. The three positions tested were: 1) melon; 2) body flank just below the lateral insertion of the dorsal fin; and 3) on the dorsal fin. To determine the best placement a short series of experiments was conducted at the Dolphin Research Center, Grassy Key, FL. Two animals were trained to station next to the trainer's platform, wear a suction cup hydrophone ('test' hydrophone; HTI-94-SSQ) placed at the test locations, and to echolocate when submerged at the test station. Due to water surface noise interference it was necessary to have the subject submerged. After the animal submerged a second hydrophone was put into the water, not attached to the dolphin, but instead $1 \mathrm{~m}$ in front of the dolphin's rostrum and approximately $5^{\circ}$ above the dolphin's horizontal midline. This position for the 'reference' hydrophone (same model as test sensor) was chosen based on previous research that has thoroughly characterized echolocation signals at that location ( $\mathrm{Au}$, 1993). The function of the reference hydrophone was to record every click produced, providing a record to which the test sensor could be compared. Both hydrophones were recorded simultaneously on a double-speed DAT recorder (Pioneer D-9601, Sampling frequency $=96 \mathrm{kHz}$ ) and monitored on a portable oscilloscope. The resulting click records were analyzed in two ways to determine the fidelity of each test location. First the tapes were played into an oscilloscope (Lecroy LC574A), click sequences were captured with a sampling frequency of $500 \mathrm{kHz}$, and the relative amplitude of each channel was calculated (Figures 1-4). In the second analysis the power spectral density of and coherence function between the two channels were compared using the 'spectrum' command in MATLAB. The power spectral density comparison evaluates the power contained in the test signal for each frequency bin, i.e. frequency-by-frequency, relative to the reference signal. The coherence function compares the phase and magnitude of the two channels (reference and test) for each frequency bin. This function was calculated using the waveform from an entire click train, and would yield a value of 1 if the spectra recorded from the two sensors were identical. With this analysis the spectrum dependent transmission of the click to the test hydrophone could be evaluated, and a ratio of transmission from reference to test locations could be approximated (Figures 5-7). 


\subsubsection{Acoustic Data Logger: Configuration and Analysis of Recordings}

The components, configuration, and operation of the acoustic data logger are fully described in Nowacek et al. (1998). Given the relatively small sample size of acoustic recordings and the level of information desired, the tapes were scored manually, i.e. no automatic detection or storage was used. A three-stage process was used to score the occurrence (including time-of-day) of each whistle and echolocation click while preserving the ICI. 1) Sound cuts ranging in duration from 5-112 minutes recorded by the data logger were digitized ( $F s=48,000 \mathrm{kHz}, 12$ bit) using the StudioTracks@ PC program initially writing to large capacity SCSI hard drives then transferred via ethernet to the working computer. 2) On the working computer (Linux based PC) MATLAB programs displayed the sound files in real-time (256-point FFT spectrograms) while I listened to the original recordings. 3) While listening to the tapes and watching the spectrograms I entered the occurrence of each click or whistle by using the computer mouse to click on the image. These stored event records contained the time of the click to within $0.0313 \mathrm{sec}$, the shortest interval that the analysis system could resolve.

Bottlenose dolphins can produce clicks with shorter ICI's than the $0.0313 \mathrm{sec}$ limit (Au, 1993), but no such high rate click trains were recorded. The results from the data logger recordings were analyzed (and displayed) in two ways for clicks; one of these methods was applied to whistle records. Method 1 calculated rates of sound production during the recording and was applied to whistle (whistles/minute) and click (clicks/second) records (e.g. Figures $8 \mathrm{a}$ and b). If the animal was sampled while still in the net corral, the release time was indicated and the mean sound production rates were calculated for the periods before and after release as well as for the entire record. The second analysis method was applied only to clicks; ICI survival plots were calculated for all available audio minutes for each animal. 


\subsection{Results}

\subsubsection{Sensor Location}

The results of the sensor placement experiments are shown in Figures 1-7. The inter-sensor calibration (Figures $1 \& 5$ ) showed the test hydrophone to be $-5.86 \mathrm{~dB}$ relative to the reference but with similar frequency response; all reported results were corrected for this discrepancy. Clicks recorded on the melon (test position \#1) averaged $+6.23 \mathrm{~dB}$ and had similar spectral content when compared to reference clicks (Figures $2 \&$ 5). At test position \#2 (body flank at the base of the dorsal fin) the test sensor recorded clicks that averaged $-19.6 \mathrm{~dB}$ relative to reference. The power spectral density comparison showed that the clicks reaching position \#2 contained much less energy in the higher frequencies with the energy dropping at approximately $5 \mathrm{~dB}$ per octave above $0.5 \mathrm{kHz}$ (Figure 6). When located on the dorsal fin (position \#3), the test sensor did not record every click produced, some clicks of relatively low intensity had an effective signal:noise ratio (SNR) of 0 (Figure 4). For more intense clicks the test sensor on the dorsal fin recorded clicks that averaged $-27.7 \mathrm{~dB}$ relative to the reference (Figure $4 \mathrm{~b}$ ). The spectral comparisons revealed a reduction in energy similar to position \#2 (Figures 6 $\& 7$ ), but the coherence between the spectral densities of the reference and position \#3 was much lower across the spectrum than for position \#2 (Figures $6 \& 7$ ). This result indicates that more of the spectrum (i.e. more frequencies) was faithfully transmitted to position \#2 than to position \#3.

\subsubsection{Data Logger Recordings}

Data loggers were attached to 13 wild animals, 2 in June 1997 and 11 in June 1998. The duration of attachment and audio recorded varied greatly among subject animals (see Table 3.1). The capability to program the tag for specific recording intervals was used to record discrete audio segments at predetermined times throughout the attachment period. The number, duration, and time of day at the start of segments 


\begin{tabular}{|c|c|c|c|c|c|c|c|}
\hline \multirow[b]{2}{*}{ Date } & \multirow[b]{2}{*}{$\begin{array}{c}\text { Focal } \\
\text { Animal } \\
\text { (Fig. \#) }\end{array}$} & \multirow[b]{2}{*}{$\begin{array}{c}\text { Pack } \\
\text { life } \\
(\mathrm{min})\end{array}$} & \multirow[b]{2}{*}{$\begin{array}{c}\text { Audio } \\
\text { Min } \\
\text { Recorded }\end{array}$} & \multicolumn{4}{|c|}{ Sound Samples } \\
\hline & & & & Segments & $\begin{array}{c}\text { Time of } \\
\text { day } \\
\text { (hrs) }\end{array}$ & Duration & $\begin{array}{l}\text { Presence of } \\
\text { clicks (c) } \\
\text { or whistles } \\
\text { (w) }\end{array}$ \\
\hline 12 June 1997 & F111 (8) & 150 & 112 & 1 & 15.6 & 112 & $c, w$ \\
\hline 13 June 1997 & FB03 (9) & 150 & 92 & 1 & 14.4 & 92 & $\mathrm{c}, \mathrm{w}$ \\
\hline 1 June 1998 & FB11 & 0.5 & 0 & & & & \\
\hline 2 June 1998 & FB90 & 1 & 0 & & & & \\
\hline & FB54 & 10 & 10 & 1 & 16.15 & 10 & \\
\hline 4 June 1998 & F115 (10) & 630 & 65 & 4 & $\begin{array}{l}18.4 \\
19.5 \\
20.5 \\
23.9\end{array}$ & $\begin{array}{c}20 \\
20 \\
20 \\
5\end{array}$ & $\begin{array}{c}c, w \\
c \\
c\end{array}$ \\
\hline 5 June 1998 & FB58 & 175 & 0 & & & & \\
\hline & $\begin{array}{l}\text { TNLV } \\
(11)\end{array}$ & 175 & 20 & 3 & $\begin{array}{l}16.1 \\
17.1 \\
18.1\end{array}$ & $\begin{array}{c}10 \\
5 \\
5\end{array}$ & $\begin{array}{l}\mathrm{c} \\
\mathrm{c} \\
\mathrm{c}\end{array}$ \\
\hline 9 June 1998 & F117 & 5 & 0 & & & & \\
\hline & FB63 & 0.5 & 0 & & & & \\
\hline & $\begin{array}{c}\text { FB09 } \\
(12)\end{array}$ & 50 & 36 & 2 & $\begin{array}{c}17.3 \\
18\end{array}$ & $\begin{array}{l}20 \\
16\end{array}$ & $\begin{array}{c}\mathrm{c}, \mathrm{w} \\
\mathrm{c}\end{array}$ \\
\hline 11 June 1998 & HSM2 & 580 & NA & & & & \\
\hline 12 June 1998 & F149 (13) & 80 & 15 & 2 & $\begin{array}{c}17.3 \\
18\end{array}$ & $\begin{array}{c}10 \\
5\end{array}$ & $\begin{array}{l}\mathrm{c}, \mathrm{w} \\
\mathrm{c}, \mathrm{w}\end{array}$ \\
\hline TOTALS & $\begin{array}{c}13 \\
\text { subjects }\end{array}$ & 2057 & 350 & 14 & & 350 & \\
\hline
\end{tabular}

Table 3.1. Tally of acoustic data logger attachments. The data logger attached to animal 'HSM2' did not include an acoustic recorder, only the ATAG (see Appendix 2). 
recorded for each animal are shown in Table 3.1. Behavioral data were collected according to the protocol described in Chapter 2. The overhead video system was not always available to sample tagged animals, and in this case continuous records of behavior visible from the observation vessel were recorded and interval samples were collected. Occurrence of notable behavioral activity were then correlated with corresponding acoustic activity recorded by the data logger.

Figures 8-13 display vocalization records for all segments listed in Table 3.1 with each animal having a unique figure number (see Table 3.1, column 2). The results for each subject are shown individually, and for the sound production rate figures each segment is shown separately with the time of day on the abscissa (Figures 8-13). If the subject was sampled before being released, the release time is indicated and sound production rates were calculated for the entire segment as well as separately for the periods before and after release. For example, for animal F115 clicks/sec were calculated for the entire segment 1 and before and after release (Figure 10a). Whistles/min were calculated similarly for the same segment (Figure 10b). For segments 2-3 no whistles were recorded, and clicks/sec are displayed in Figures 10c\&d; if applicable, click and whistle records for the same segment are ordered sequentially (e.g. Figures 10a\&b). Time of day is reported in decimal hours, not clock minutes. Finally, ICI survival plots for all segments combined were calculated and are shown last in a given animal's series (e.g. Figure 10e). ICI survival plots display the ICI duration (abscissa) and the number of ICI's recorded (ordinate) that were of that duration or longer. Each individual for which clicks or whistles were recorded has a unique figure number (Table 3.1), but the number of figures differs by animal due to number of segments recorded and presence/absence of clicks or whistles in a particular segment (Table 3.1). 
For echolocation clicks two features are present throughout the data, and a third is less common but noteworthy. The most striking feature is the occurrence of intermittent bursts of clicks, i.e. the animals produced many clicks at a time but these events occurred sporadically through the records. This characteristic is noticeable both in the time series records (e.g. Figure 8a) as discrete histogram bars of many clicks/sec separated by sometimes long periods of no clicks, and in the ICI survival plots showing that there are often $10^{1}$ or $10^{2}$ more clicks spaced at the shortest intervals than at intermediate or longer ICI's (e.g. Figure 8c). In addition, the occurrence of irregular and often numerous relatively long ICI's demonstrates that packets can be spaced by tens of minutes (e.g. Figure $8 \mathrm{c}$ ). The second interesting feature is the overall low rate of click production, the calculation of which is dominated by the long periods with no click production. Thirdly, animals sometimes produced a small number of clicks (1-5) within one second and did not click at all during the surrounding seconds (e.g. Figure 10d). These clicks often occurred within 5 seconds of the animal surfacing (35\%) or diving after a surfacing (26\%). Surfacing events were unambiguous in the data logger record as the hydrophone exited and re-entered the water. Finally, sound production rates before release from temporary capture were greater than after release, often by more than $10^{1}$ for both clicks (e.g. Figure 10a) and whistles (e.g. Figure 8b). In all cases for both types of sound, the rate of production was greater before release than after.

\subsubsection{Behavioral Correlates}

The behavioral activity during notable periods of acoustic activity showed some interesting but inconclusive correlates. For example, during the time period around 17:00 hours F111 (Figures $8 \mathrm{a} \& \mathrm{~b}$ ) clicked and whistled at higher rates than for the surrounding hour. At 16.9 hours (decimal hours, i.e. 0.1 hours $=6$ minutes) she was observed 'pinwheeling' (see Section 4.3) at the surface, and only 5 minutes later F111 and her dependent calf crossed paths but did not interact with 2 other animals (no surface interaction was observed). Unfortunately, at a time of relatively high click rate (16.116.2 hours) F111 was not visible from the surface. 
FB03 produced a number of click packets during the half-hour from 15-15.5 (Figure 9a). During this time she was alone (i.e. no other animals within $1 \mathrm{~km}$ ) and swimming in and out of sea grass beds in shallow water ( $<2 \mathrm{~m}$ depth). The edges of seagrass beds are areas of significant foraging activity (Section 4.3.4).

FB54 was released with her dependent calf, and the two swam rapidly away from the net but did not vocalize or encounter any other animals during the 10 minute sample. The two whistled regularly while in the net corral, but not after the data logger was attached.

F115 also had a dependent calf at the time of the experiment. F115 whistled just around the time of release, occasionally during the first recording segment (Figure 10b), but not at all during segments $2-4$. She clicked during all but the last segment, although at a low rate. F115 and her calf did not encounter any other animals during the recording period $(2100 \mathrm{hrs})$. The pair spent the entire experiment period slowly traveling back and forth along the shoreline in a corner of a small bay. The only observed behavior other than the slow travel was a possible nursing bout seen from the overhead video camera (see Appendix 1) during which no sounds were recorded (17.75). The pair continued this pattern of slow travel for $>24$ hours after the data logger was recovered as observed by other vessels involved in the project.

TNLV was released with another male, FB58, but was observed only during the first recorded segment because the two animals separated and FB58 was followed (acoustic record not recovered). FB09 was released with her dependent calf, the two traveled across some sand and seagrass covered shallows during the first 20 minutes after release, and then traveled down a channel for the rest of that segment and the subsequent one. They did not encounter any other animals during the recording period. Finally, F149 was released also with a dependent calf. She did not vocalize until she whistled as 
the two traveled a short distance across sandy shallows and into the channel. During the second segment the pair was traveling in the Inter-Coastal Waterway (ICW) and were at the confluence with a smaller channel with no other animals in sight.

\subsection{Conclusions and Discussion}

Recording rates of sound production in wild cetaceans has been a long-standing problem for researchers. Due to technical problems associated with identifying the signaler and, in the case of echolocation, a narrow transmit beam pattern, reliably recording vocalization rates has eluded researchers. The current work presents data recorded by a novel research tool that recorded every echolocation click and whistle produced by individual free-ranging bottlenose dolphins. From these data reliable rates of sound production are reported for seven Sarasota dolphins. The most notable result is the low rate of both whistle and echolocation click production for free-ranging animals. Clicks and whistles occurred primarily in bursts separated by relatively long periods of silence.

Bottlenose dolphin clicks are typically produced in bursts or trains, and they can be divided into two production pattern categories. First, those clicks produced with sufficient ICI to allow for the return and processing of one click before the next is emitted, i.e. the ICI is greater than the sum of the two-way travel time between the dolphin and the target and some processing time. Second, some clicks are produced with ICI's less than the two-way travel time, i.e. the echo from one click has not returned before the next click is emitted. Both types of click patterns, long and short ICI, have been reported for bottlenose dolphins as well as other species (Au, 1993; Ivanov \& Popov, 1979; Norris et al., 1961; Turl \& Penner, 1989). The current results can not be used in support of either argument because range to target is unknown. The majority of recent research has reported that bottlenose dolphins use exclusively long ICI click trains (Au, 1993), but at very close range $\mathrm{Au}$ (1993) states that dolphins are likely processing more than one echo at a time. Miller (1995) reported two distinct stages of echolocation having significantly different ICI's during presumed feeding in narwhals, but they could 
not assign signals to individuals nor confirm that the narwhals were feeding. No such stages of echolocation were recorded during the current study.

The fact that dolphins use clicks to detect and/or discriminate objects is not at issue, but the emission pattern of a given click train may not always be dictated by target range. Norris (1961), for example, reported that click repetition rate in their experiments was not strictly linked to the target range. Click production rates from 2 clicks/sec $[\mathrm{ICI}=0.5$, approximate target range (assuming $20 \mathrm{~ms}$ lag time) $=360 \mathrm{~m}]$ up to almost 25 clicks $/ \mathrm{sec}$ [ICI=0.04 $\mathrm{sec}$, approximate target range (assuming $20 \mathrm{~ms}$ lag time) $=15 \mathrm{~m}$ ] were measured in the current study. It is not unreasonable that a dolphin might be assessing a target at $15 \mathrm{~m}$, but $360 \mathrm{~m}$ is well beyond the demonstrated detection range for bottlenose dolphins ( $\mathrm{Au}, 1993$ ) suggesting a different function for the low rate clicks.

These low rate or single clicks often produced in association with surfacing events may fulfill a specific ranging need. Gordon and Tyack (1999) speculate that sperm whales echo-range off the bottom and surface to determine their depth. Perhaps bottlenose dolphins in Sarasota Bay are using these clicks to judge their depth; at least two other possibilities are conceivable. If traveling in the opaque waters of a channel in Sarasota (5-10 m depth) a dolphin may need to verify that there is not a boat at the spot where it needs to surface. There are often many boats in the channels so even the noise of a moving boat may not be enough to localize an open patch of water for surfacing. The second possibility is that upon re-entry into the water after a surfacing, a dolphin might use one or a few widely spaced clicks to ascertain not only depth but also some bathymetric characteristics (e.g. sand or seagrass bottom ). Such habitat information is important for these animals (see Section 4.3.4), and a dolphin can certainly discriminate between echoes of clicks from such different targets (see (Au et al., 1995 and Au, 1993 \#26) for dolphin discrimination capabilities). 
The low overall rate of click production may be surprising if the traditional roles for echolocation are correct. Historically the presumption has been that clicks are involved in food finding and navigation. Given that only one possible foraging event was observed (F111 at 17 hrs., Figure 8a) and most of the recordings were made during a relatively short time after the capture-release process the low click rates are not surprising. Alternatively, given that some of the different foraging modes involve flushing prey from obvious refuges (Chapter 4), perhaps dolphins do not need to use echolocation to detect prey in these circumstances. The click rates of two animals (FB03 and FB09) when in important foraging habitats were among the highest rates recorded (Figures $9 \mathrm{a}$ and $12 \mathrm{a}$, respectively). This result may indicate some foraging use of echolocation in these habitats, but as no foraging was observed the animals could also have been clicking to navigate out of the shallows or perhaps no prey were detected. In regards to navigation, the only other behavior observed was traveling. If dolphins depend on echolocation to navigate, then many more clicks would be expected. Consider, however, the abilities of echolocating bats. Bats have excellent spatial memories. When flying in familiar settings they either cease to produce sonar sounds or fail to listen to the echoes of their sound because they collide with newly introduced obstacles (Griffin, 1958; Holler, 1995; Neuweiler \& Mohres, 1966). The dolphins sampled in the current study are residents of Sarasota Bay, and the youngest animal sampled was 8 years old. These dolphins may, therefore, have developed a spatial map of the area, which may reduce the need for navigational clicks. Updating that map may, however, be important. F149 clicked at a relatively high rate when at the confluence of two channels, and she also whistled throughout the click bouts (Figures 13c and d). Perhaps she vocalized to orient herself and/or coordinate her movements with her calf.

The engineering design of the dolphins' biosonar system is well understood ( $\mathrm{Au}$, 1993), but there is little information regarding the ecological use of echolocation by wild dolphins. The current results demonstrate that the click patterns produced by wild dolphins can be recorded, and such data have the potential to provide insight into the 
ecology of dolphin echolocation. Studying echolocation in free-ranging dolphins is an integral component in contributing to our understanding of the use and function of this highly developed sense. A combination of controlled experiments with captive dolphins involved in biologically relevant tasks and studies of dolphins in their natural environment will likely reveal a great deal about the ecology of echolocation.

Due to the complexity of the environment and demands on a given individual, it is difficult to assess the circumstances that influence when and to what extent an individual produces echolocation. One constraint on echolocation is the presumed energetic cost of producing echolocation signals. Arita (1997) suggested that the costs of flight and echolocation are high for bats. The relative costs of locomotion are likely to be much lower in swimming dolphins than flying bats, but the costs of echolocation for dolphins are unknown. Additional constraints include the potential detection of echolocation signals by predators and/or prey. While only few fish species have been shown to possess the ability to detect echolocation type signals (Astrup \& Mfhl, 1993; Dunning et al., 1992) or frequencies (Mann, Lu \& Popper, 1997), many prey species have not yet been tested for this ability. This lack of knowledge leaves an open question regarding the potential costs of an echolocating dolphin being detected by its prey. The benefits of using echolocation include the ability to orient during navigation and to detect and possibly discriminate prey. These needs may, however, be somewhat reduced by the use of other senses to accomplish these tasks. Vision, for example, appears to be relatively well developed in dolphins (Dawson, 1980; Guofu \& Kaiya, 1992; Murayama \& Somiya, 1998) so during the day their reliance on echolocation may be reduced. The ecological costs and benefits of echolocation are, therefore, at least theoretically complex.

Combining controlled experiments with observations of free-ranging animals has been a successful formula for elucidating the ecology of bat echolocation. With the ability to record echolocation from dolphins in their natural environment and continued controlled study of captive or restrained dolphins, perhaps we can begin to explore the natural ecology of dolphin echolocation. 
a)

$-5.78 \mathrm{dBV}$

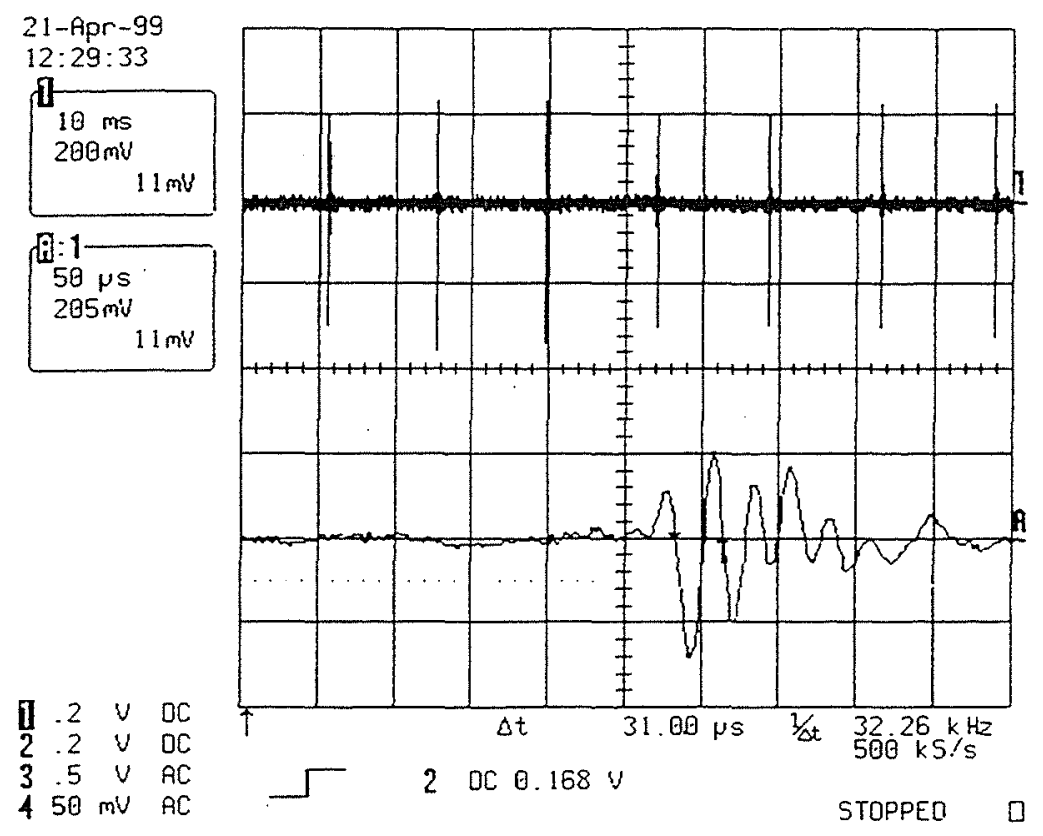

b)

$-10.17 \mathrm{dBV}$

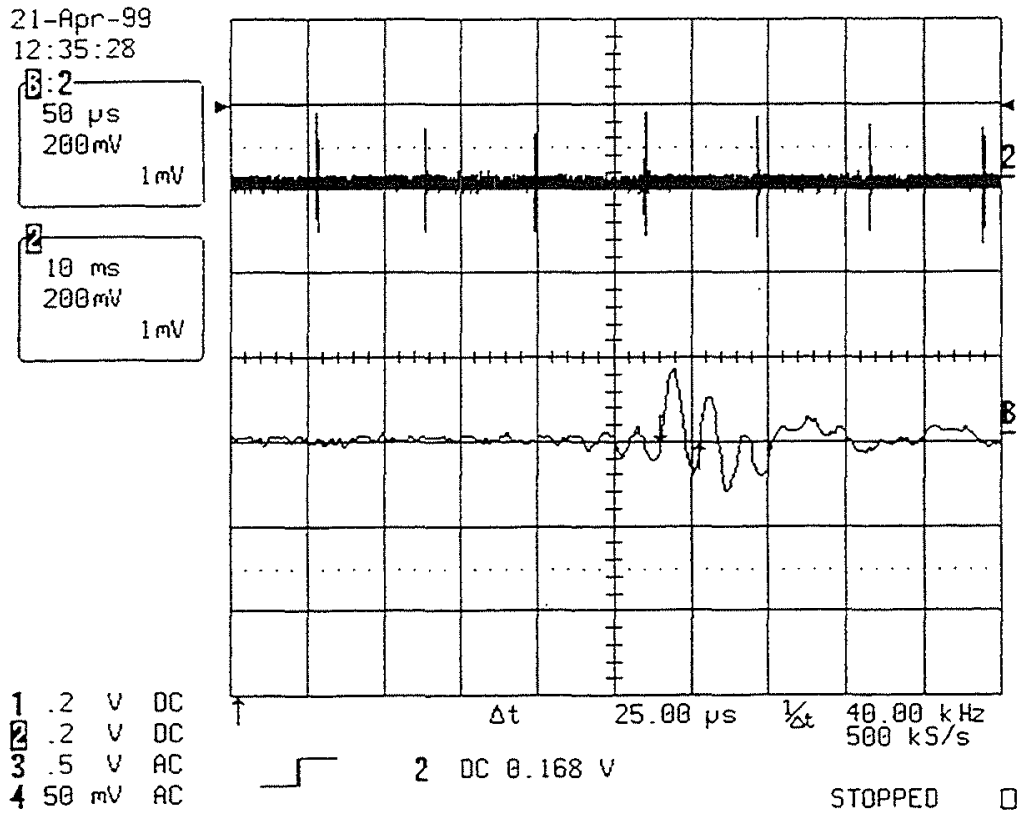

Figure 1. Inter-hydrophone calibration. Both the reference (a) and test (b) hydrophones are $1 \mathrm{~m}$ in front of the animal. A click train and a 'zoomed-in' waveform of one click are shown for (a) the reference hydrophone (top) and (b) test hydrophone. Average relative amplitudes (dB referenced to IV) are shown with figure sub-label, (a) or (b), for clicks recorded at the corresponding location. 
a)

$-7.83 \mathrm{dBV}$

b)

$0.13 \mathrm{dBV}$
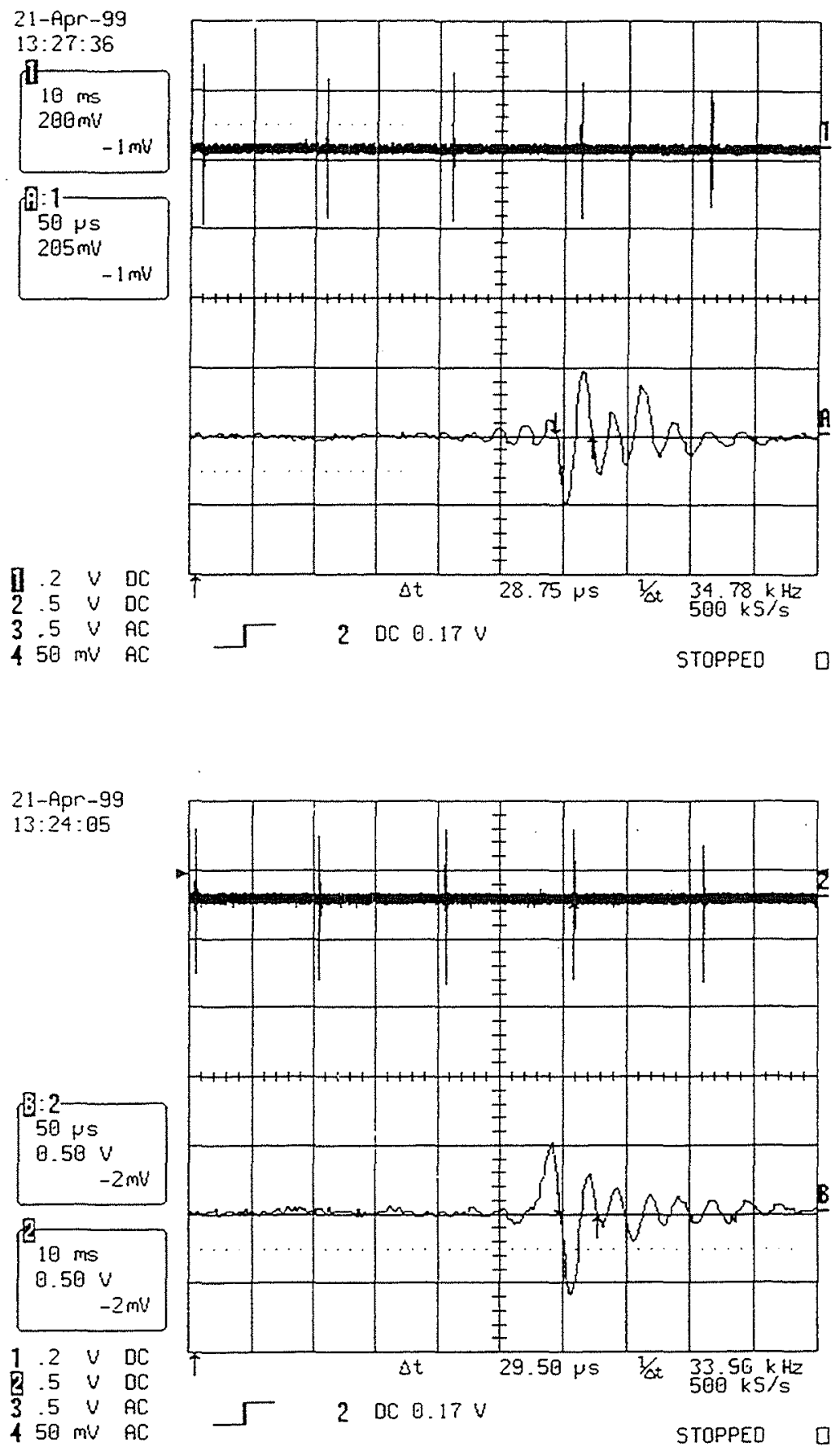

Figure 2. Click waveforms recorded by (a) the reference hydrophone at the $1 \mathrm{~m}$ reference position, and by (b) the test sensor at test position \#1, the melon. A click train and a 'zoomed-in' waveform of one click are shown for (a) the reference hydrophone (top) and (b) test hydrophone. Average relative amplitudes ( $\mathrm{dB}$ referenced to $\mathrm{V}$ ) are shown with figure sub-label, (a) or (b), for clicks recorded at the corresponding location. 
a)

$2.35 \mathrm{dBV}$

b)

$-20.35 \mathrm{dBV}$
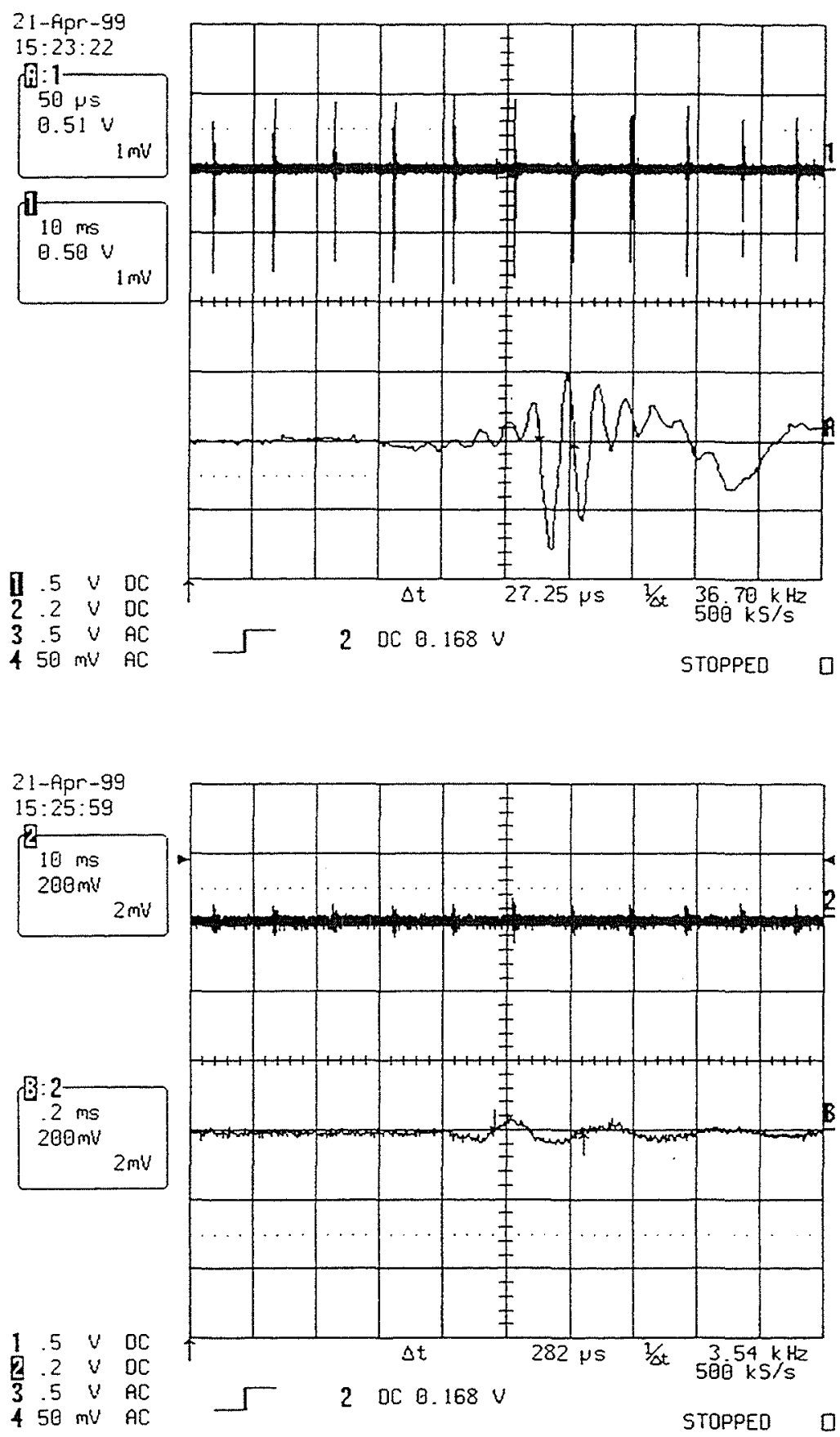

Figure 3. Click waveforms recorded by (a) the reference hydrophone at $1 \mathrm{~m}$, and (b) the test hydrophone at test position \#2, the body at the base of the dorsal fin. A click train and a 'zoomed-in' waveform of one click are shown for (a) the reference hydrophone (top) and (b) test hydrophone. Average relative amplitudes ( $\mathrm{dB}$ referenced to $\mathrm{IV}$ ) are shown with figure sub-label, (a) or (b), for clicks recorded at the corresponding location. 
a)

$8.88 \mathrm{dBV}$

$-18.86 \mathrm{dBV}$
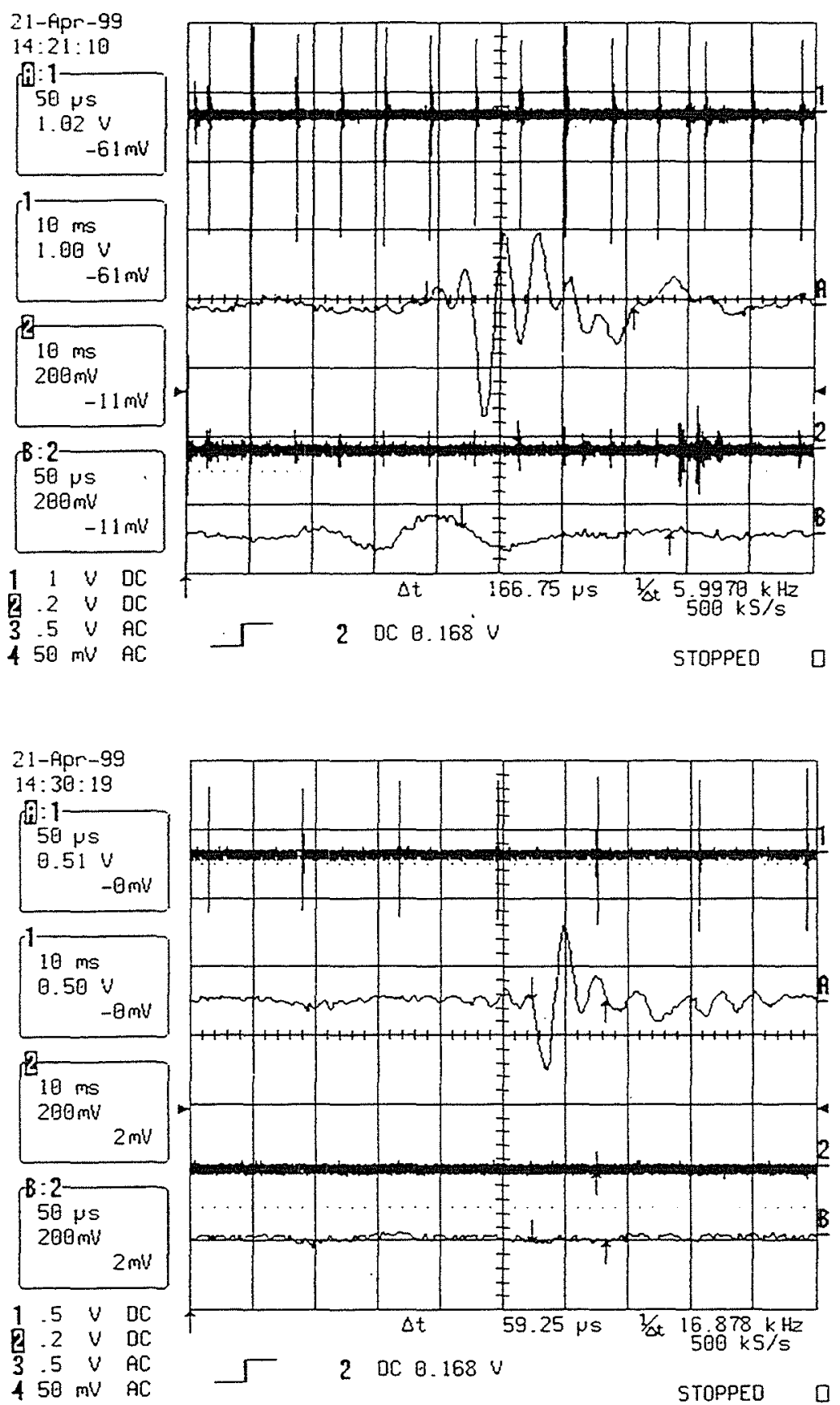

b)

\section{$0.91 \mathrm{dBV}$ undetectable}

Figure 4. Two sets of click waveforms recorded by the reference hydrophone at $1 \mathrm{~m}$ and test hydrophone at test position \#3, on the dorsal fin. A click train and a 'zoomed-in' waveform of one click are shown in (a) and (b). In (a) and (b) the top set of two waveforms (click train and 'zoomed-in' click) was recorded by the reference hydrophone, and the lower set by the test hydrophone. Average relative amplitudes ( $\mathrm{dB}$ referenced to $1 \mathrm{~V}$ ) are shown with figure sub-label, (a) or (b), for clicks recorded at the corresponding location. The relative amplitude for the reference hydrophone is shown first. 

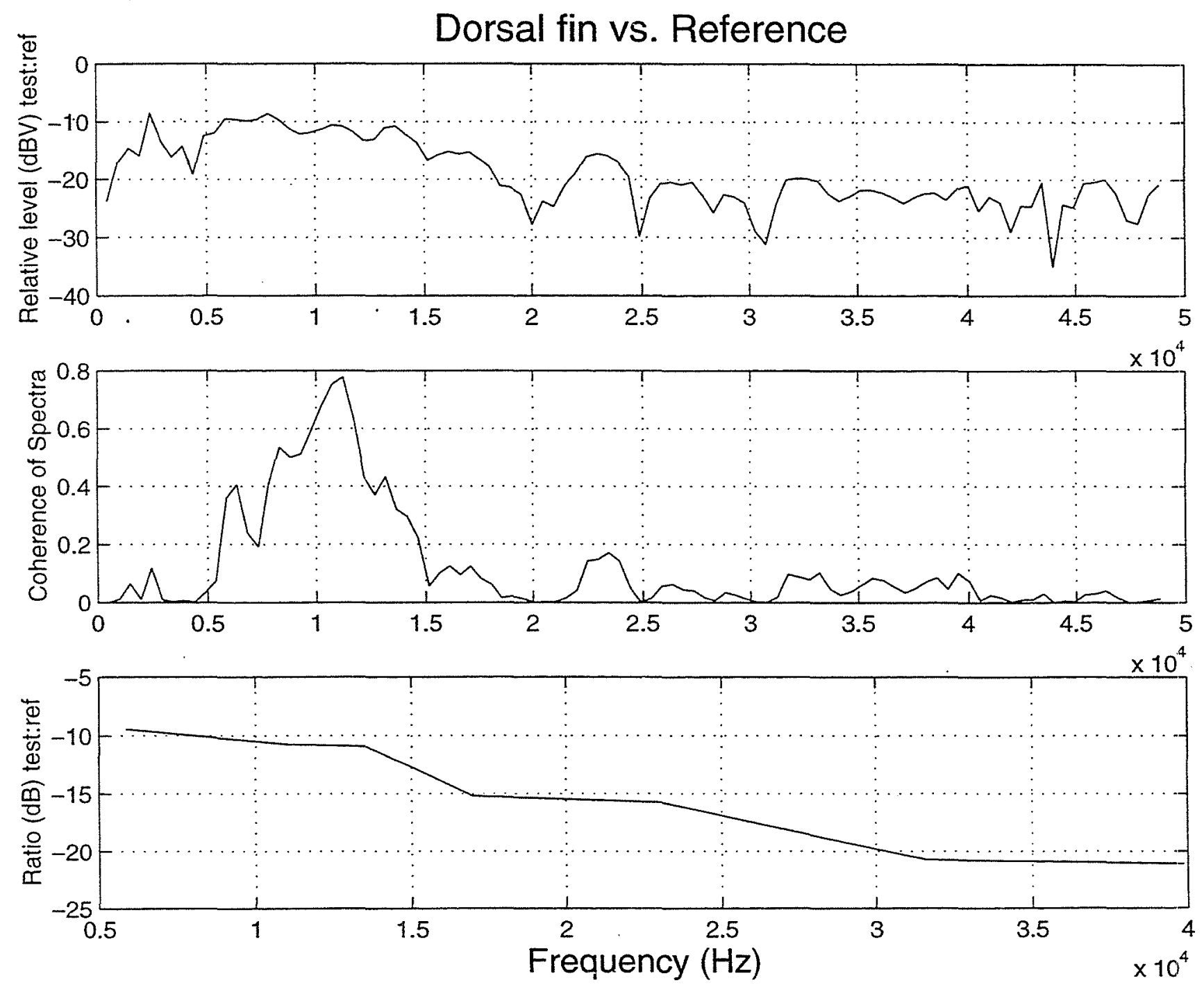

Figure 7. Spectral comparison of signals from the reference hydrophone and test hydrophone at position \#3, on the dorsal fin. The power spectral density of the test hydrophone relative to the reference (top), the coherence function between the two channels (middle), and the ratio (dBV) of the test hydrophone compared to the reference (bottom). See text for explanation of power spectral density and coherence. 

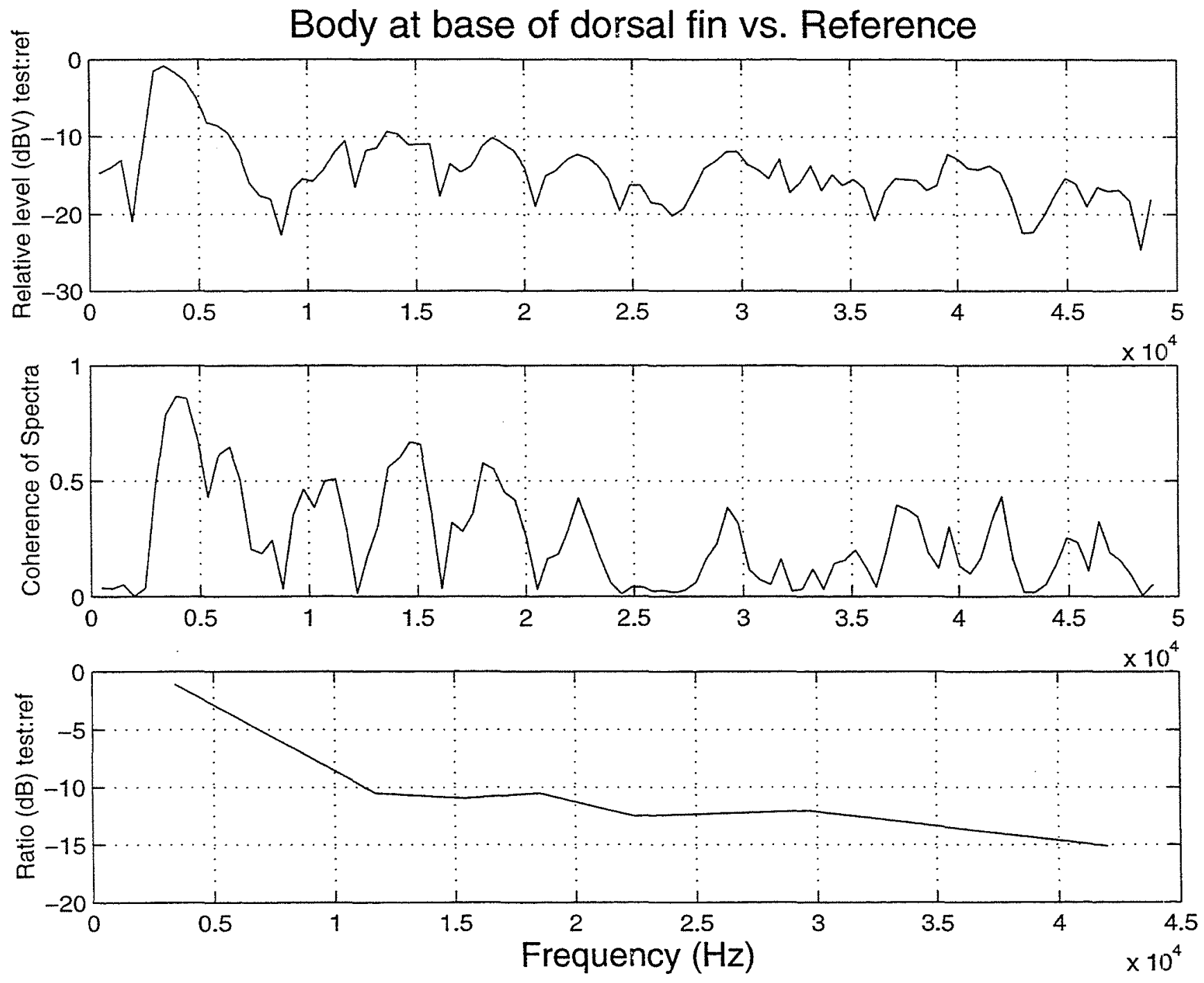

Figure 6. Spectral comparison of signals from the reference hydrophone and test hydrophone at position \#2, on the body at the base of the dorsal fin. The power spectral density of the test hydrophone relative to the reference (top), the coherence function between the two channels (middle), and the ratio ( $\mathrm{dBV}$ ) of the test hydrophone compared to the reference (bottom). See text for explanation of power spectral density and coherence. 

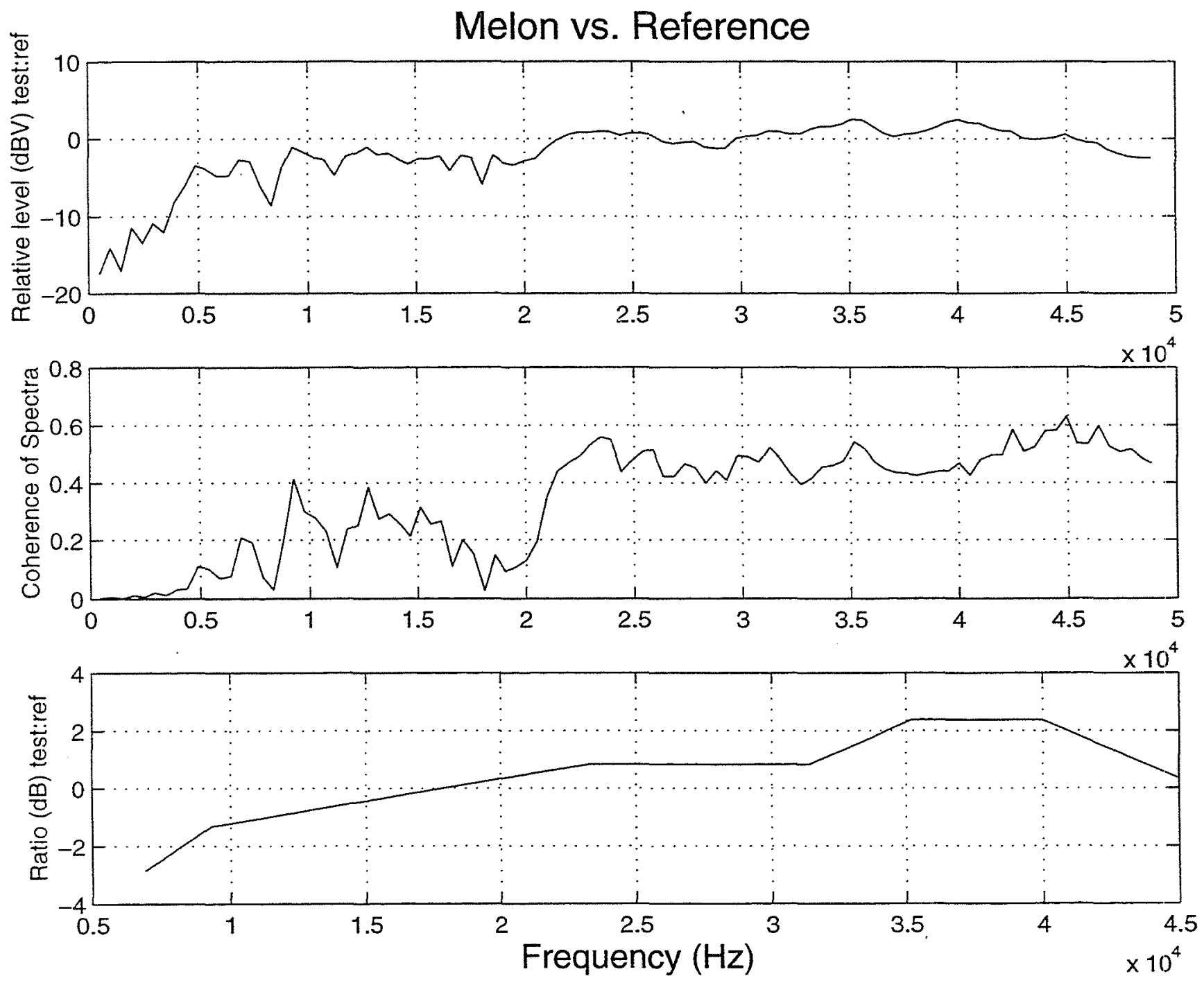

Figure 5. Spectral comparison of signals from the reference hydrophone and test hydrophone at position \#1, the melon. The power spectral density of the test hydrophone relative to the reference (top), the coherence function between the two channels (middle), and the ratio $(\mathrm{dBV})$ of the test hydrophone compared to the reference (bottom). See text for explanation of power spectral density and coherence. 


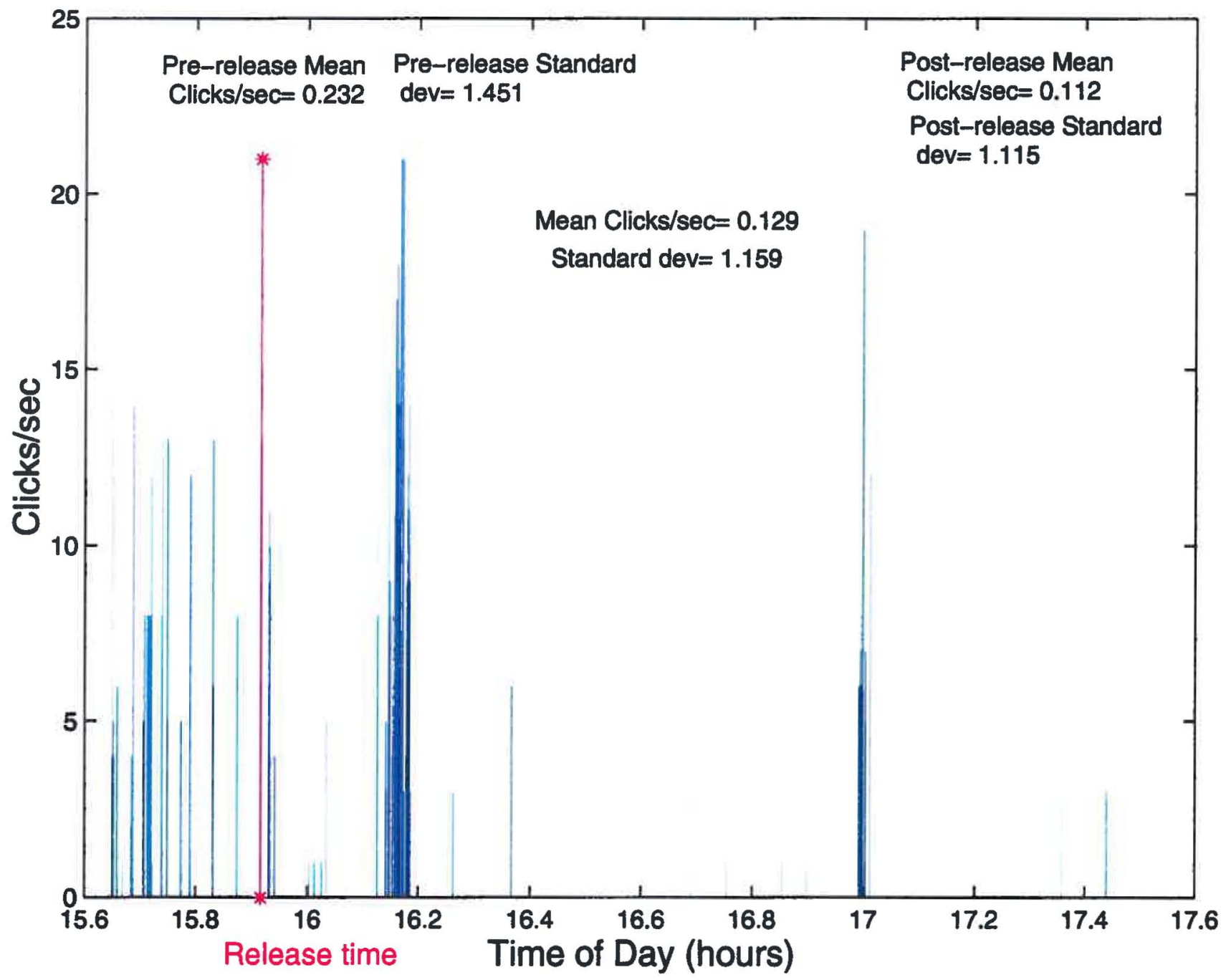

Figure 8a. Click rate record for F111. Mean clicks/second and standard deviation are shown in the center of the figure for the entire 112-minute record as well as in the top corners for the periods before and after release. 'Release time' is indicated by the red line bounded with asterisks. 

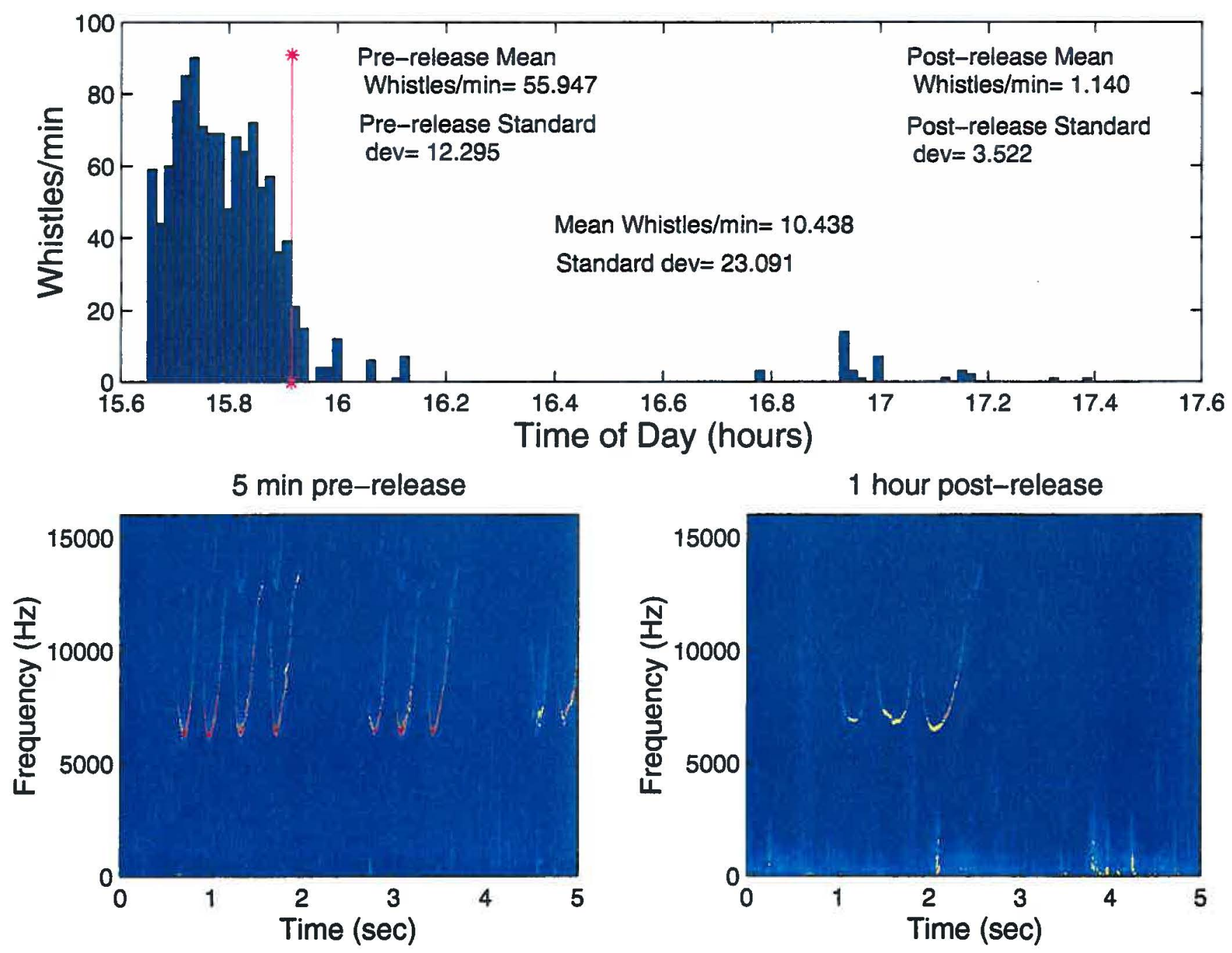

Figure 8b. Whistle rate record for F111 (top) and samples of whistles from pre-release (lower left) and 1-hour post-release (bottom right). Mean whistles/min and standard deviation are shown in the center of the top figure for the entire 112-minute record as well as in the top corners for the periods before and after release. 'Release time' is indicated by the red line bounded with asterisks. The two whistle samples show that F111 used time-warped versions of her signature whistle pre and post-release. 


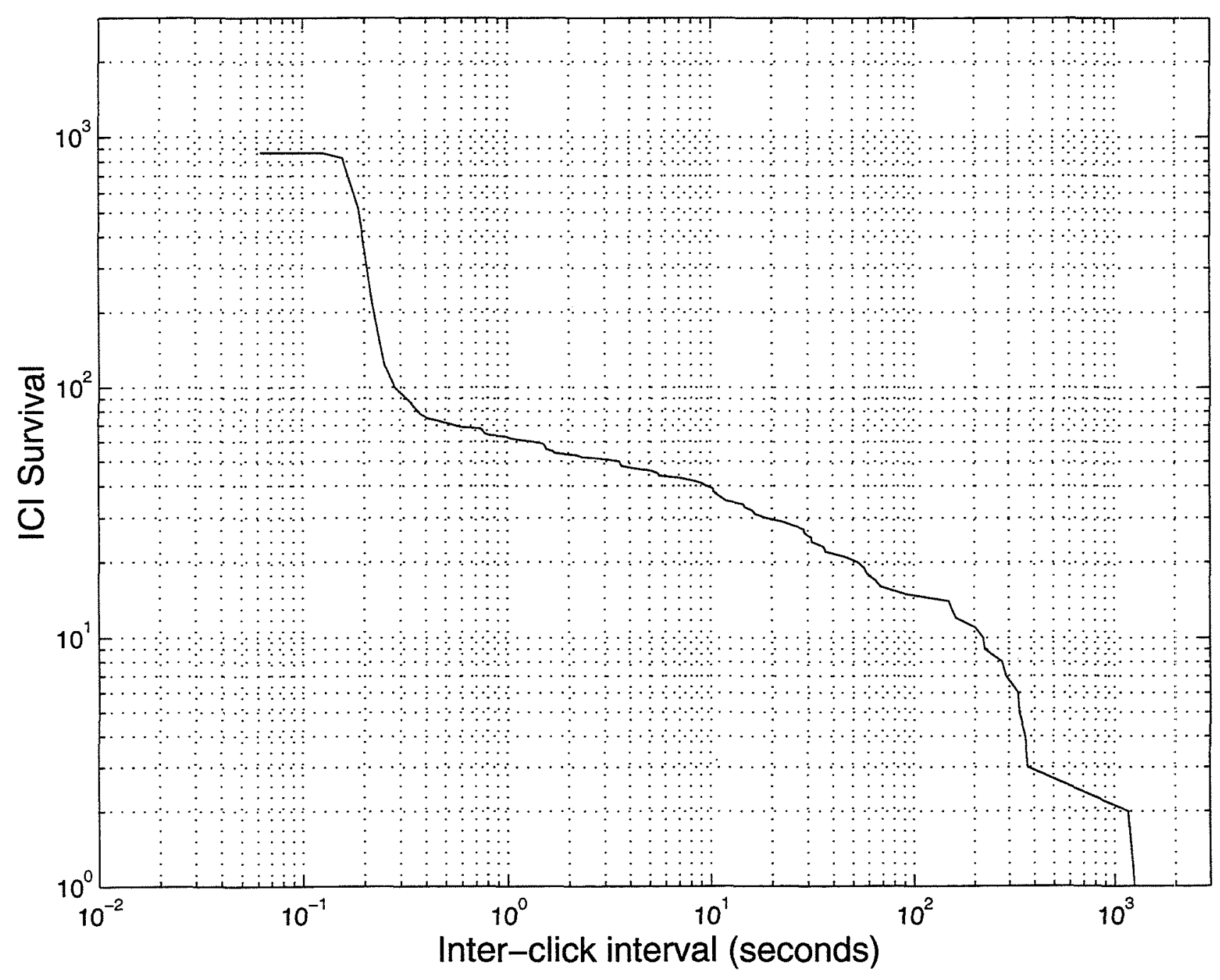

Figure 8c. Log-log survival plot of all inter-click intervals for F111. For each ICI duration on the abscissa the value of the ordinate indicates the number of ICI's recorded that are of that duration and longer. 


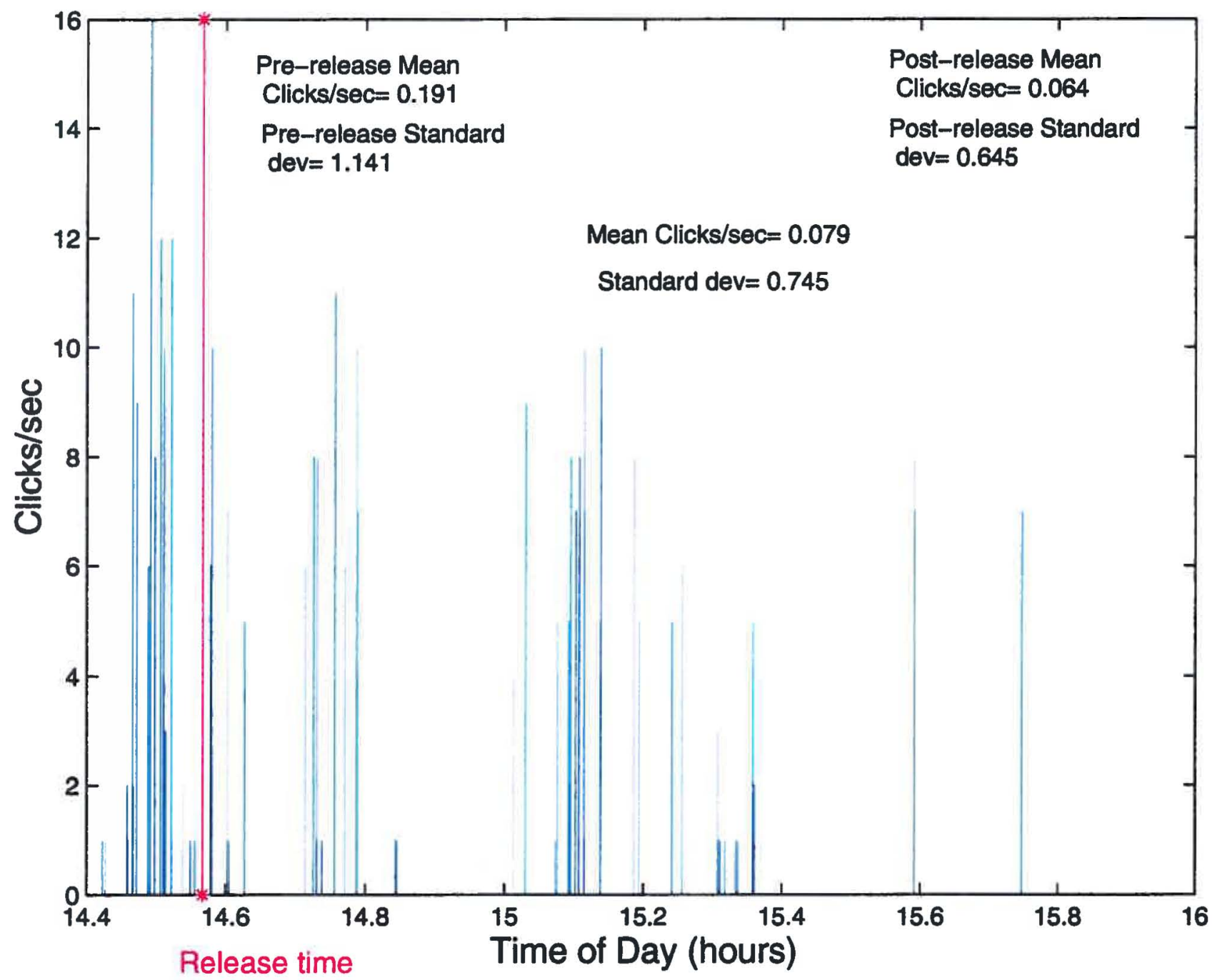

Figure 9a. Click rate record for FB03. Mean clicks/second and standard deviation are shown in the center of the figure for the entire 92-minute record as well as in the top corners for the periods before and after release. 'Release time' is indicated by the red line bounded with asterisks. 


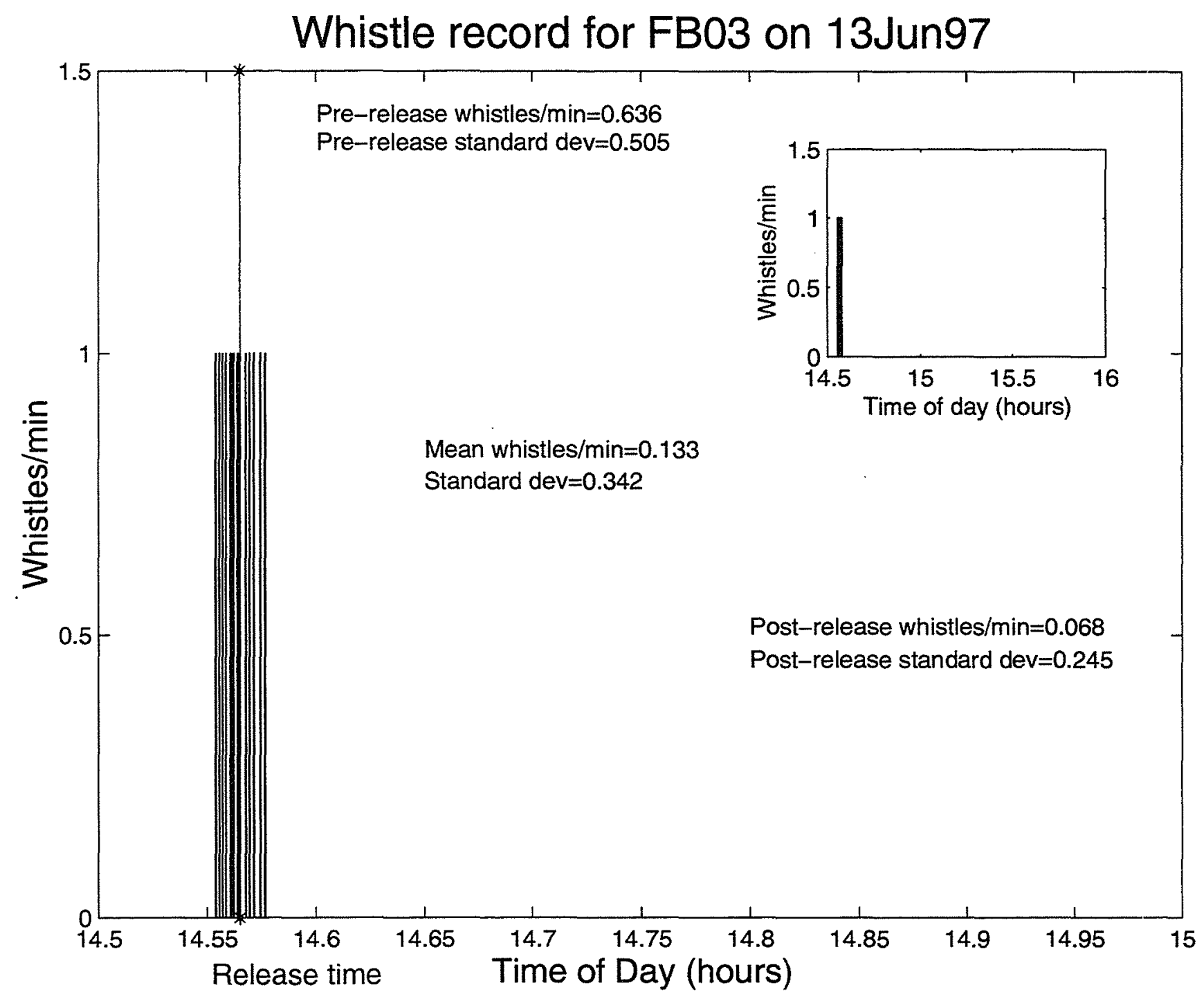

Figure $9 \mathrm{~b}$. Whistle rate record for FB03. The main figure shows only a short part of the entire record around the time of release for better histogram resolution. The inset shows the entire record; FB03 did not whistle after the few emitted around the time of release. Mean whistles/min and standard deviation are shown in the center of the figure for the entire 92-minute record as well as in the top corners for the periods before and after release. 'Release time' is indicated by the red line bounded with asterisks. 


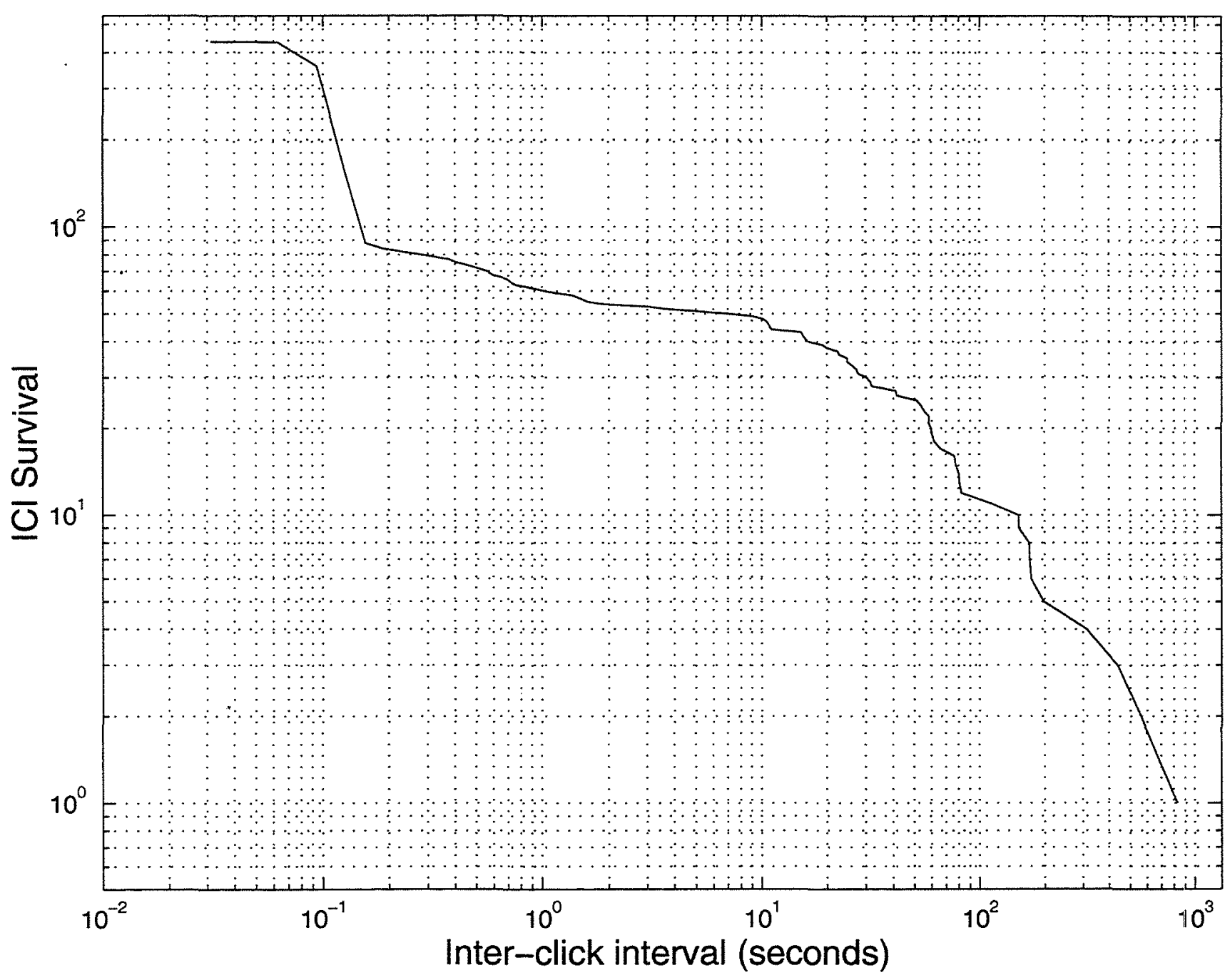

Figure 9c. Log-log survival plot of all inter-click intervals for FB03. For each ICI duration on the abscissa the value of the ordinate indicates the number of ICI's recorded that are of that duration and longer. 


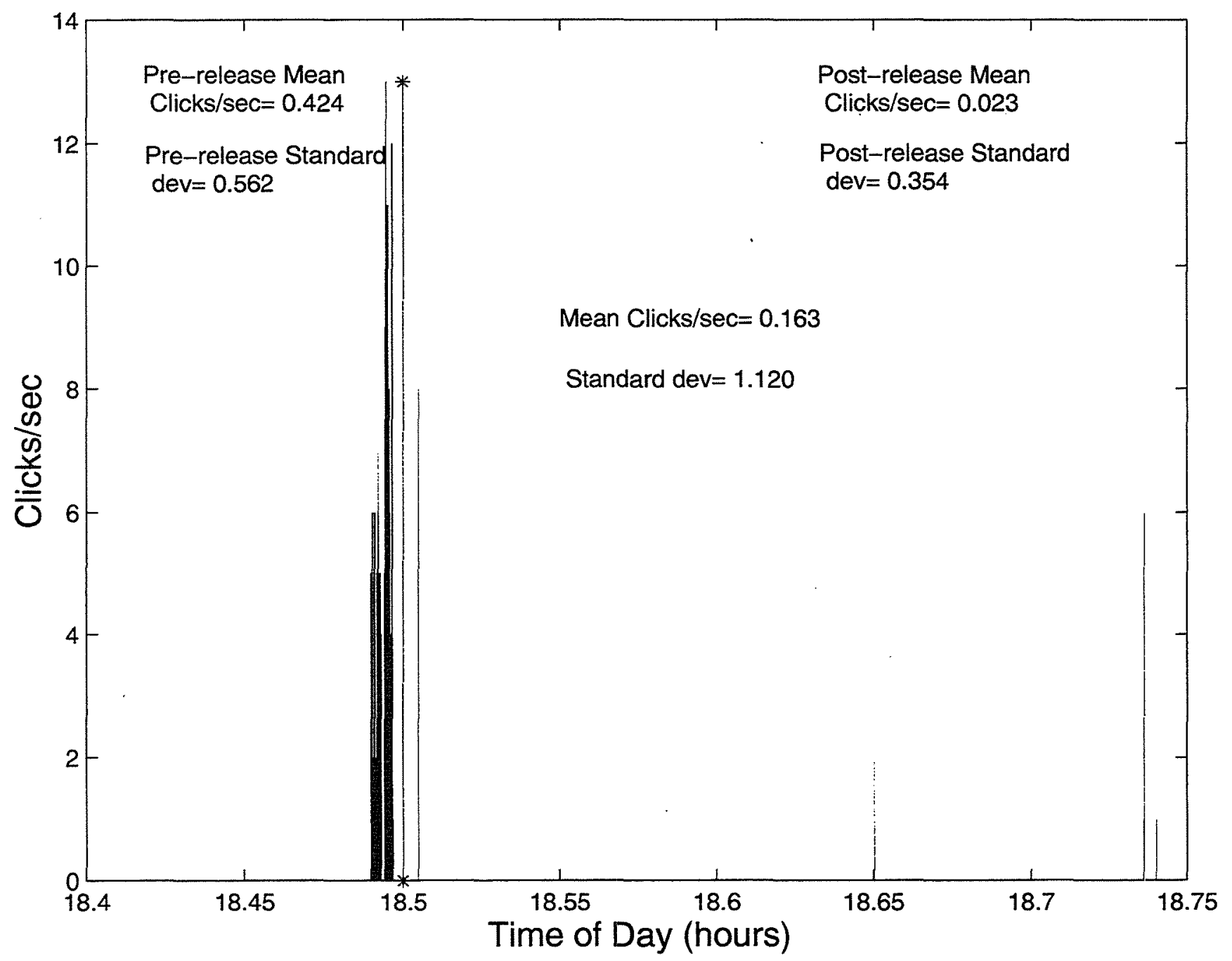

Figure 10a. Click rate record for F115 segment 1. Mean clicks/second and standard deviation are shown in the center of the figure for the 20-minute segment as well as in the top corners for the periods before and after release. 'Release time' is indicated by the red line bounded with asterisks. 


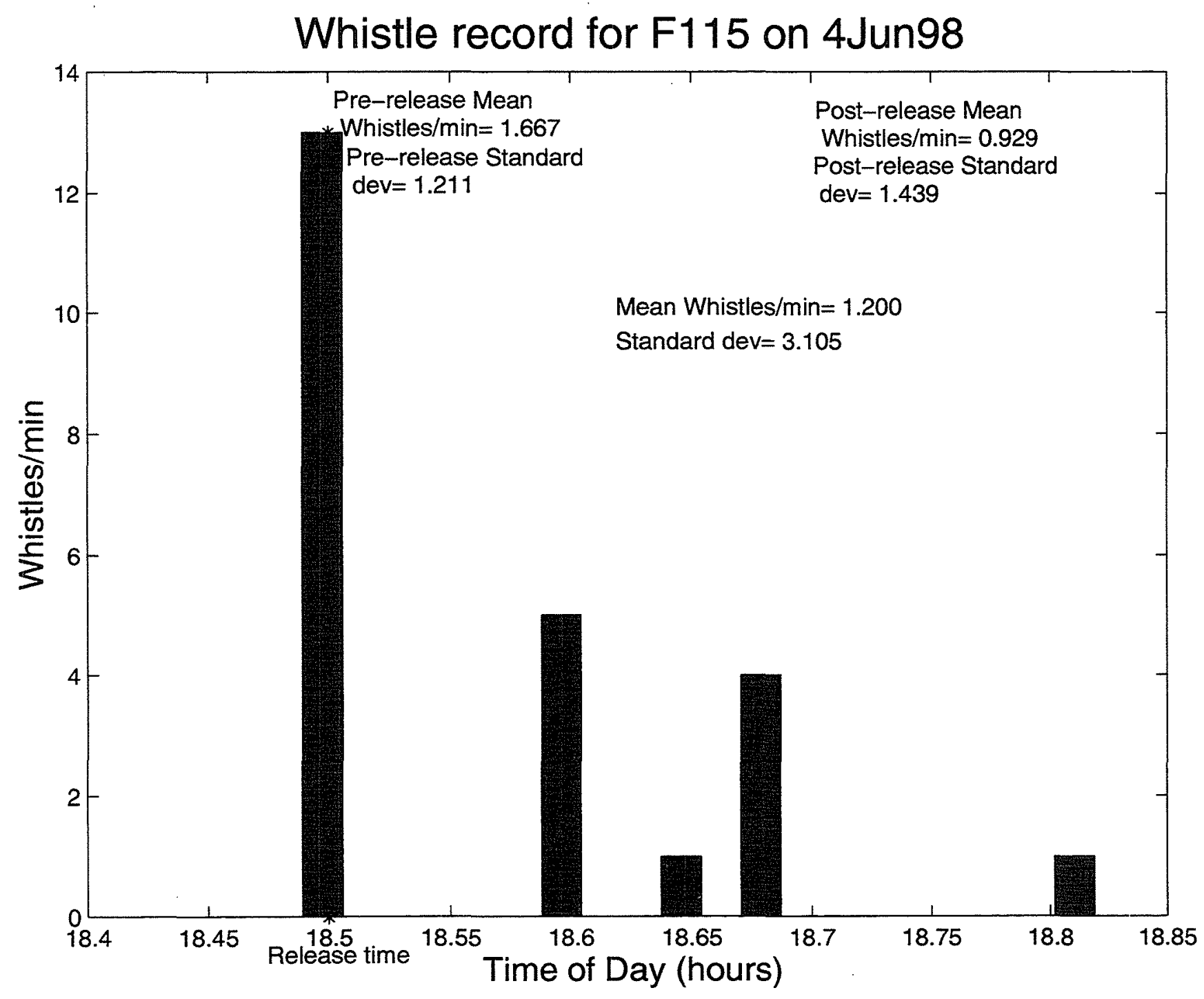

Figure 10b. Whistle rate record for F115 segment 1. Mean whistles/minute and standard deviation are shown in the center of the figure for the 20 -minute segment as well as in the top corners for the periods before and after release. 'Release time' is indicated by the red line bounded with asterisks. 


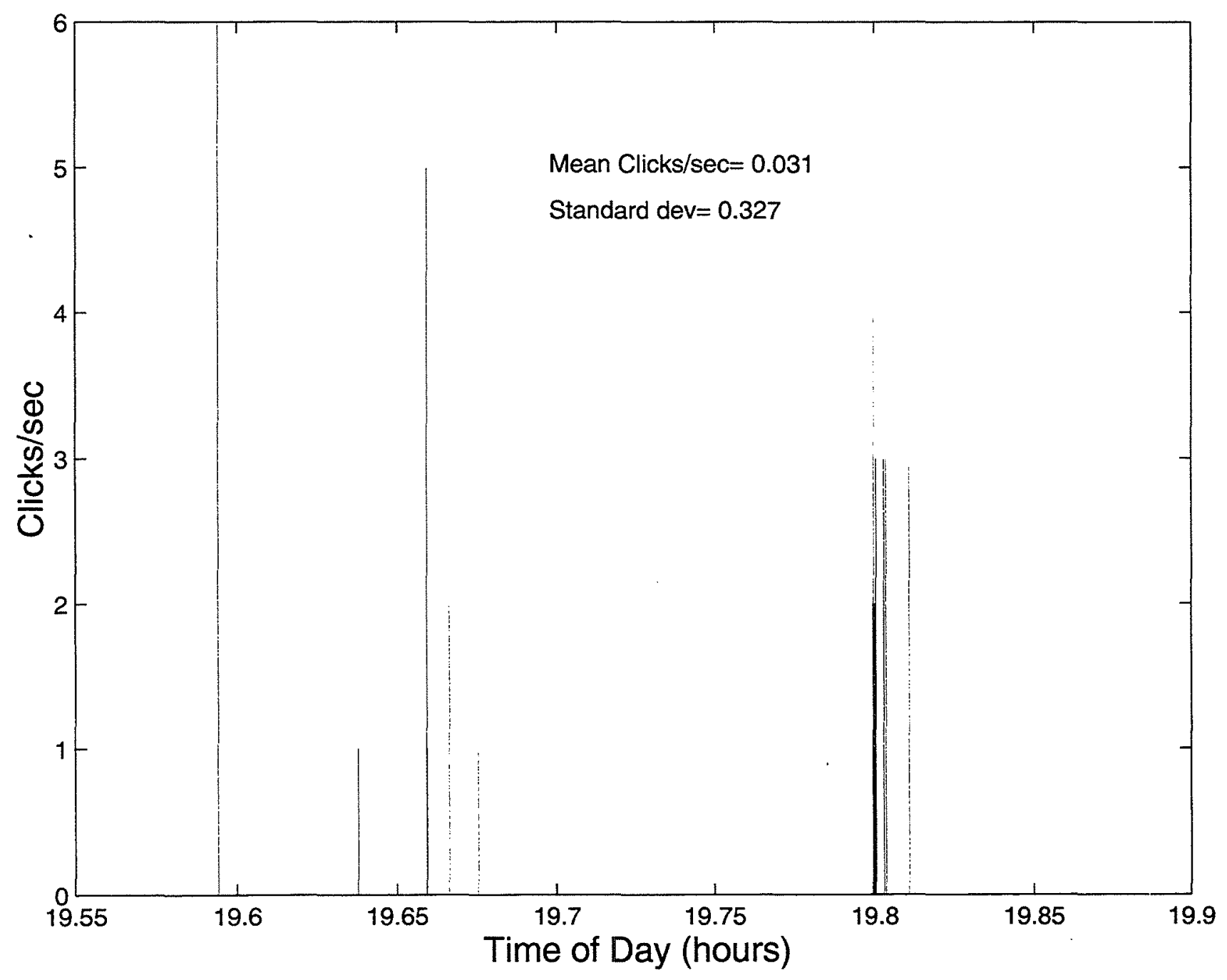

Figure 10c. Click rate record for F115 segment 2. Mean clicks/second and standard deviation are shown in the center of the figure for the 20-minute segment. This segment began 1 hour post-release. 


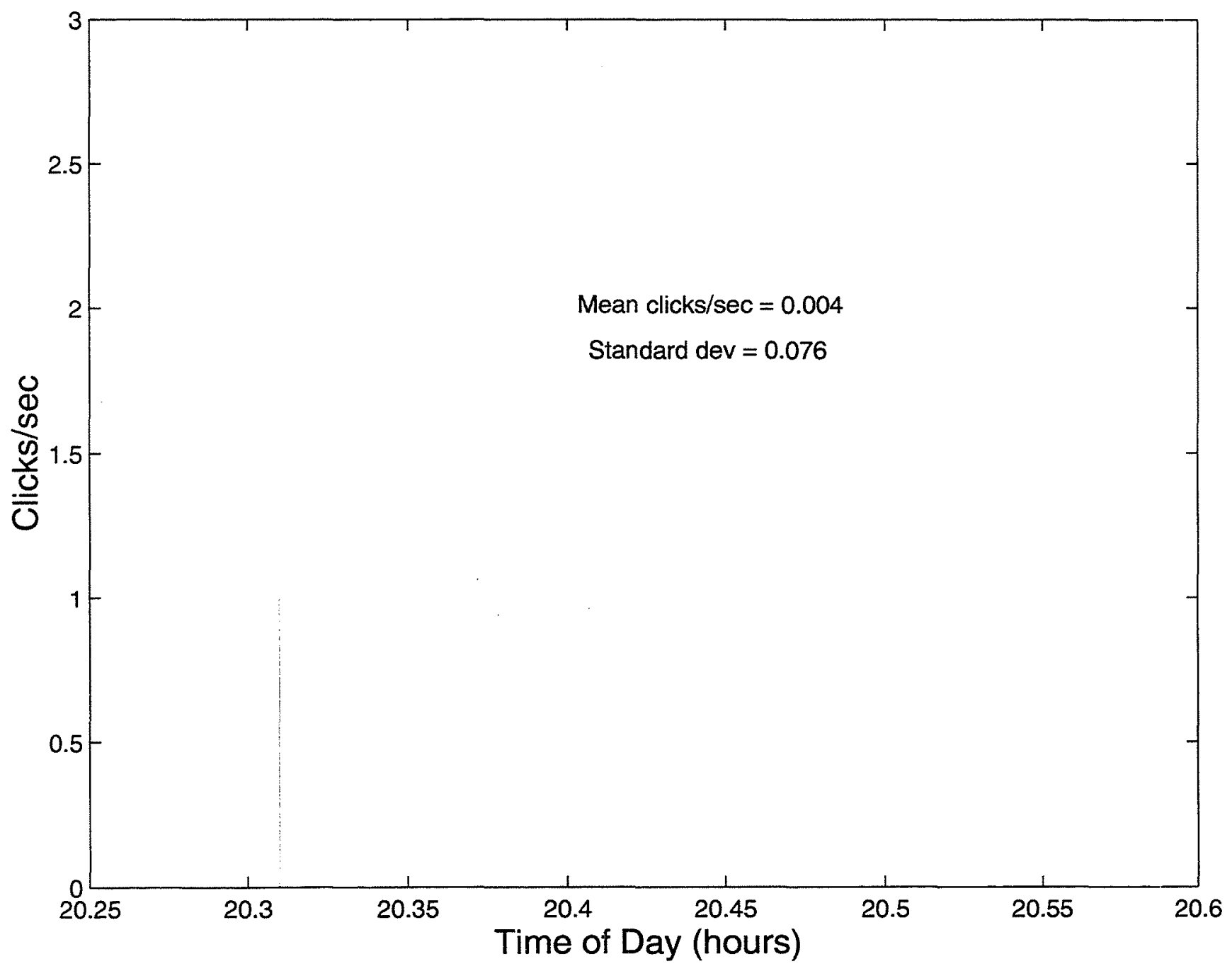

Figure 10d. Click rate record for F115 segment 3. Mean clicks/second and standard deviation are shown in the center of the figure for the 20-minute segment. This segment began 2 hours post-release. 


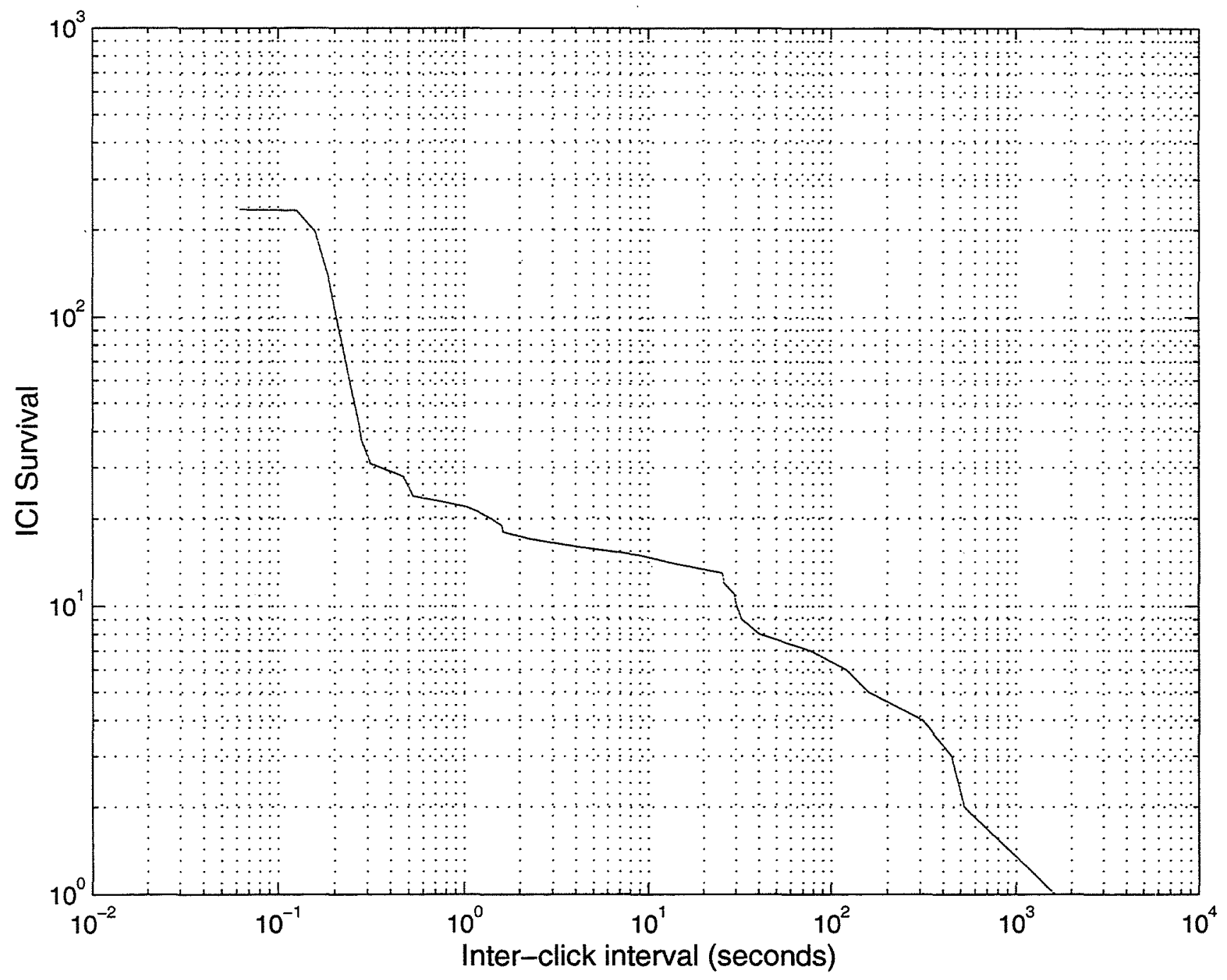

Figure 10e. Log-log survival plot of all inter-click intervals for F115. For each ICI duration on the abscissa the value of the ordinate indicates the number of ICI's recorded that are of that duration and longer. 


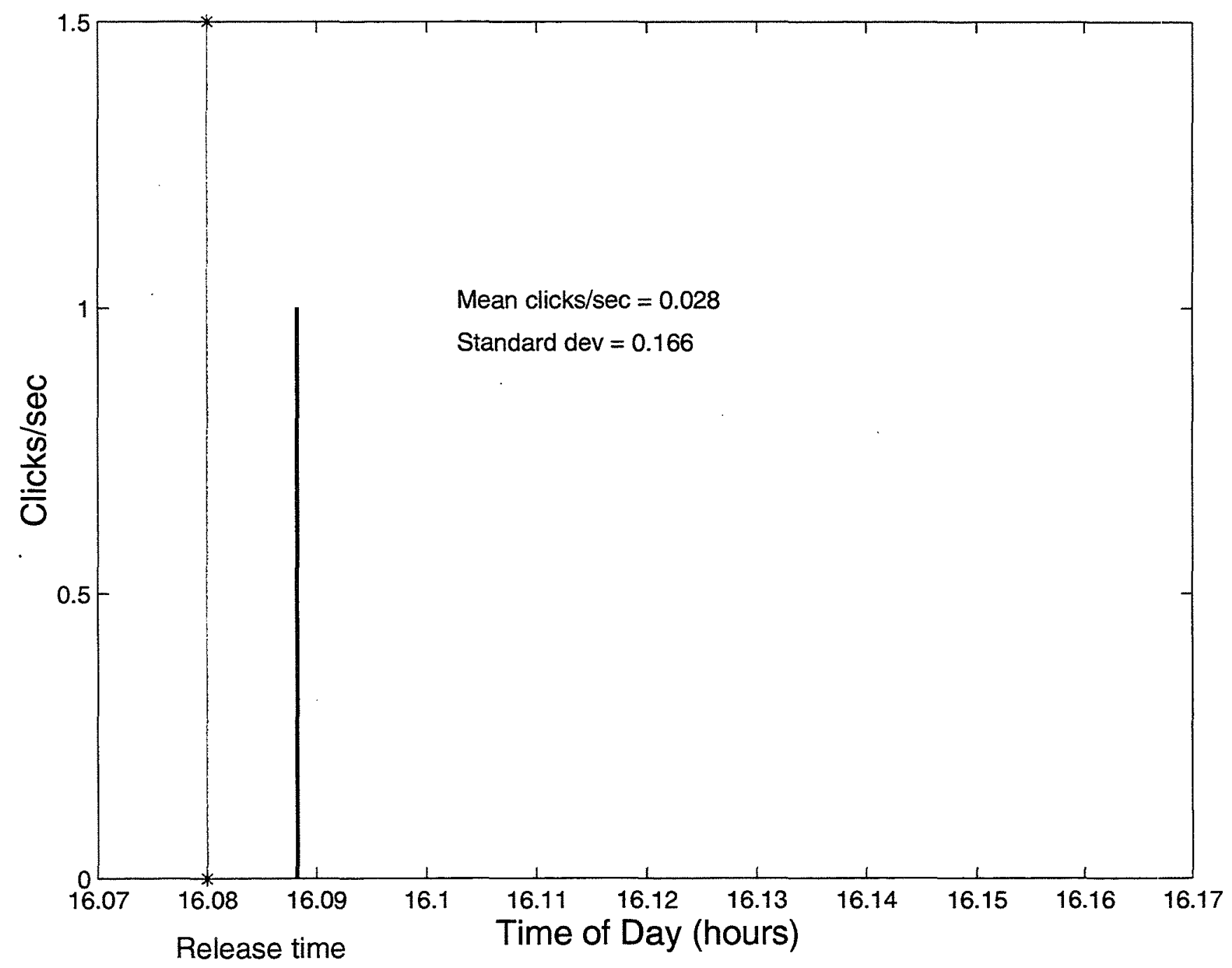

Figure 11a. Click rate record for TNLV segment 1. Mean clicks/second and standard deviation are shown in the center of the figure for the 10-minute segment. 'Release time' is indicated by the red line bounded with asterisks. 


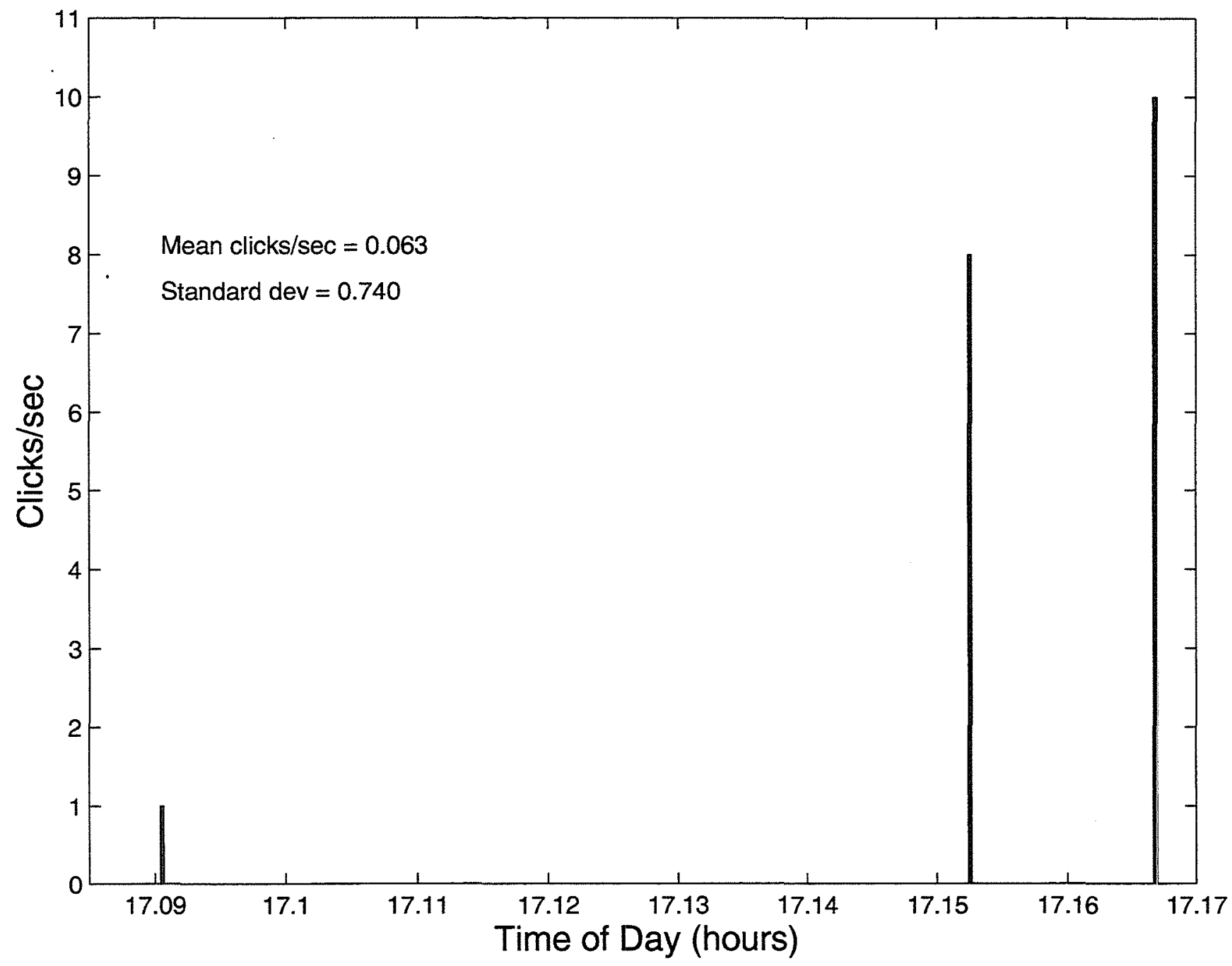

Figure 11b. Click rate record for TNLV segment 2. Mean clicks/second and standard deviation are shown in the center of the figure for the 5-minute segment. 


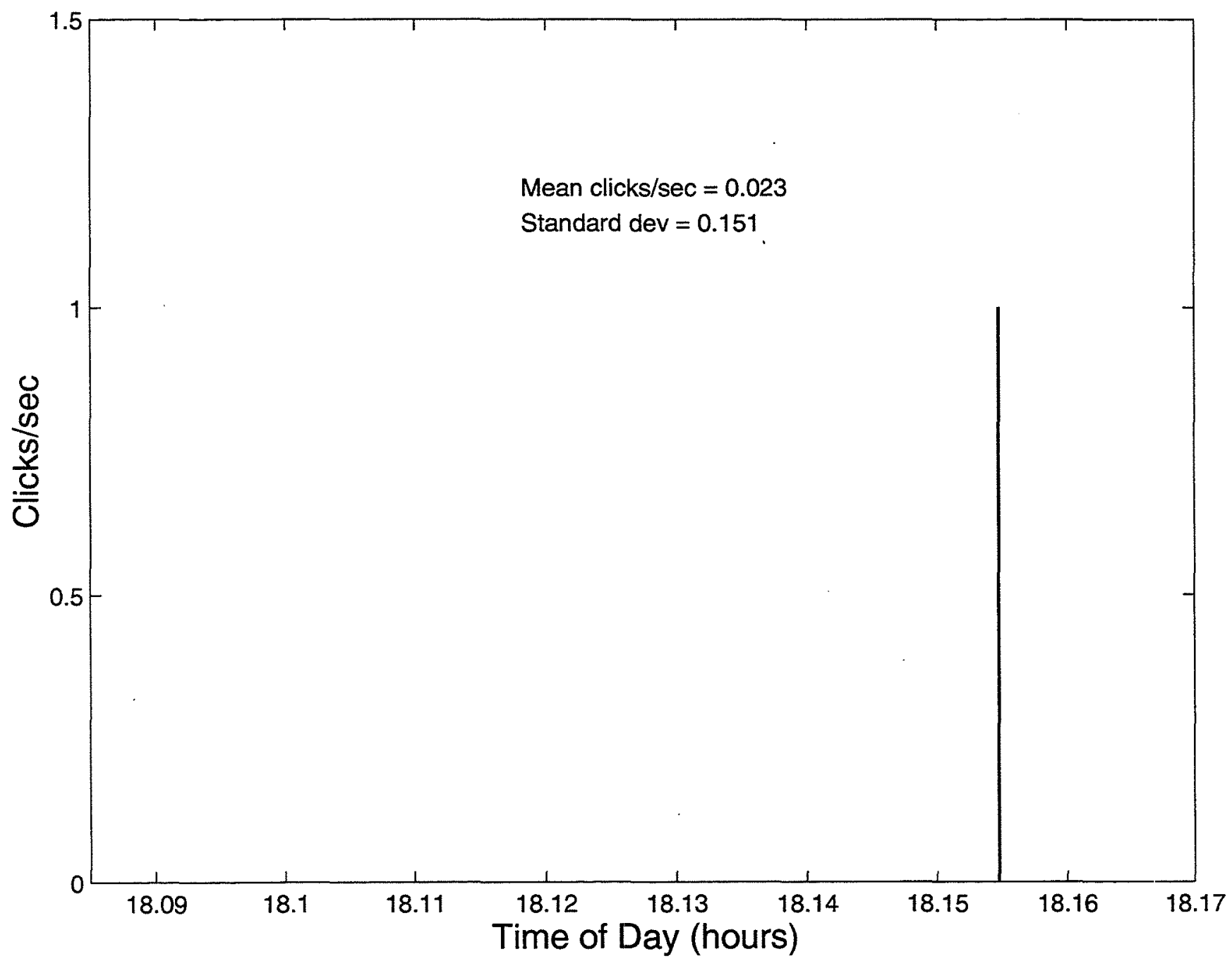

Figure 11c. Click rate record for TNLV segment 3. Mean clicks/second and standard deviation are shown in the center of the figure for the 5-minute segment. 


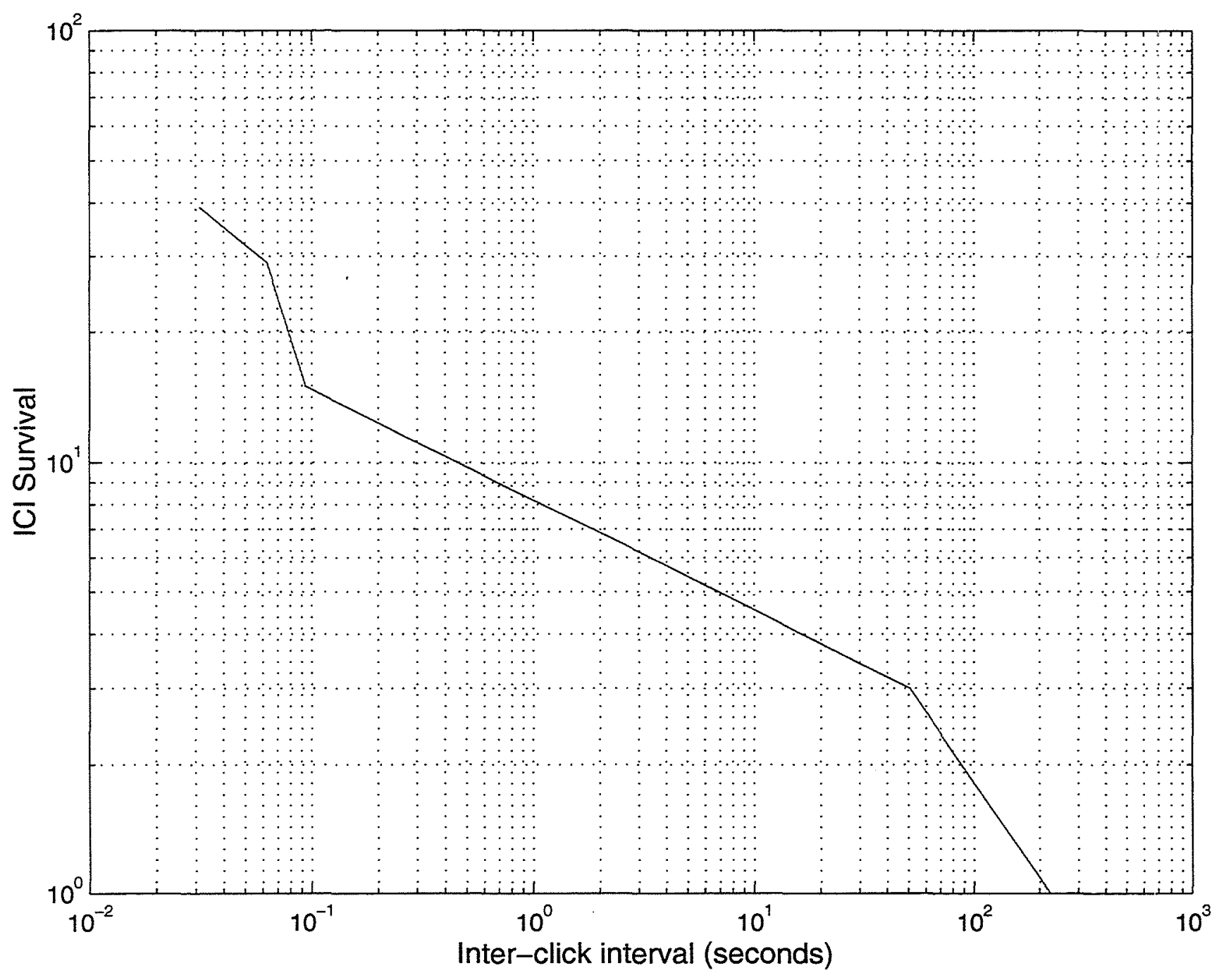

Figure 11d. Log-log survival plot of all inter-click intervals for TNLV. For each ICI duration on the abscissa the value of the ordinate indicates the number of ICI's recorded that are of that duration and longer. 


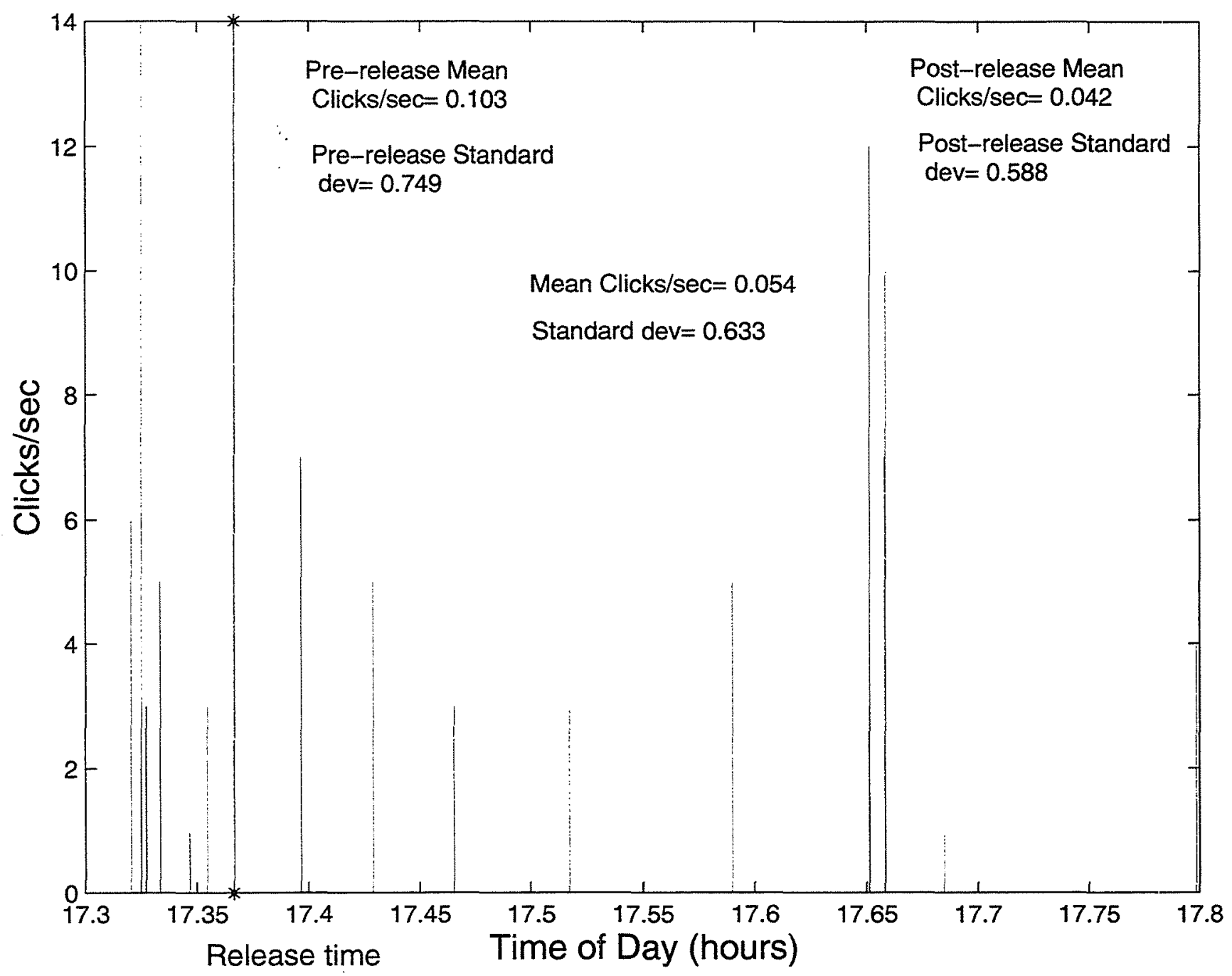

Figure 12a. Click rate record for FB09 segment 1. Mean clicks/second and standard deviation are shown in the center of the figure for the 20-minute segment as well as in the top corners for the periods before and after release. 'Release time' is indicated by the red line bounded with asterisks. 


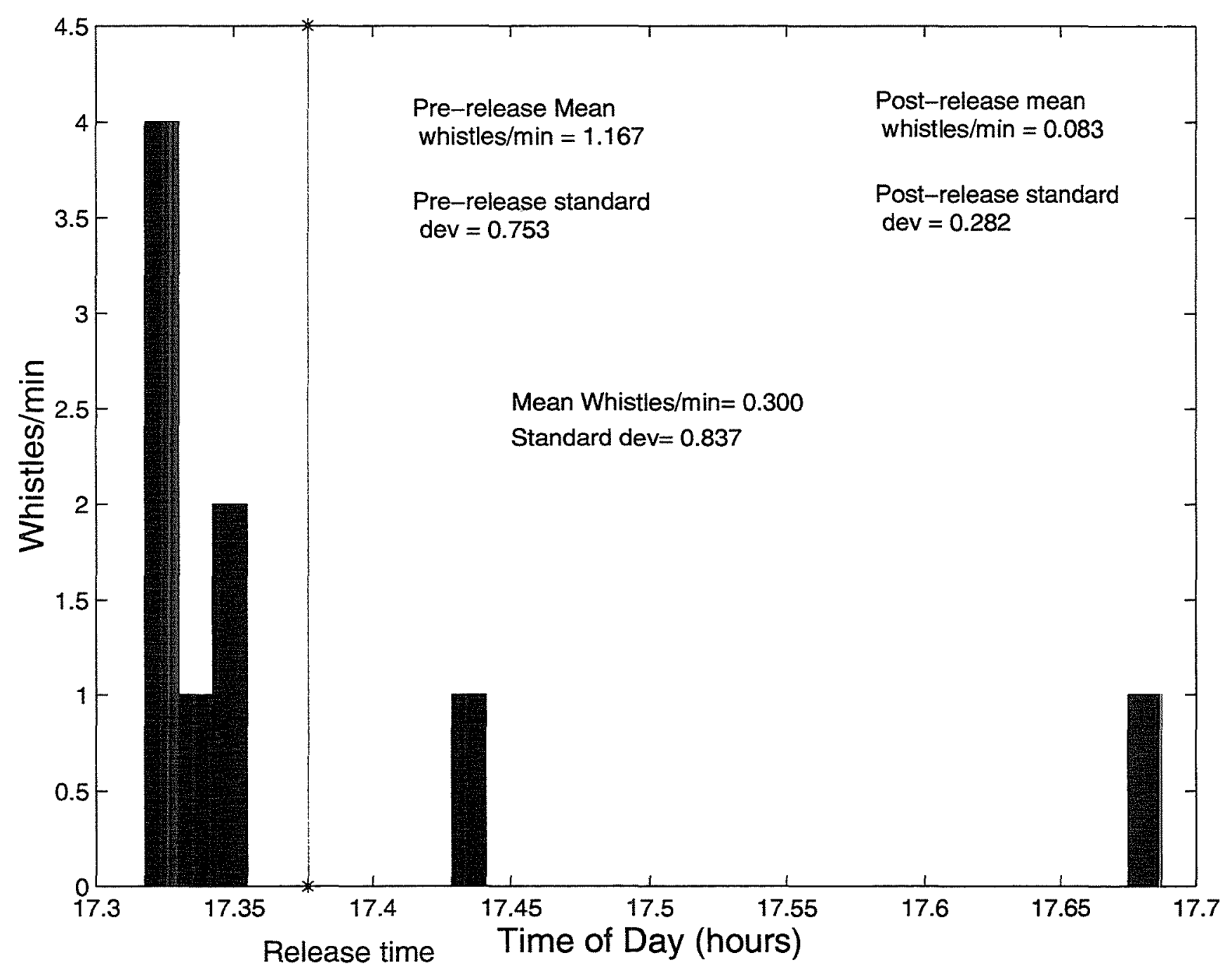

Figure 12b. Whistle rate record for FB09 for segment 1. Mean whistles/minute and standard deviation are shown in the center of the figure for the 20-minute segment as well as in the top corners for the periods before and after release. 'Release time' is indicated by the red line bounded with asterisks. 


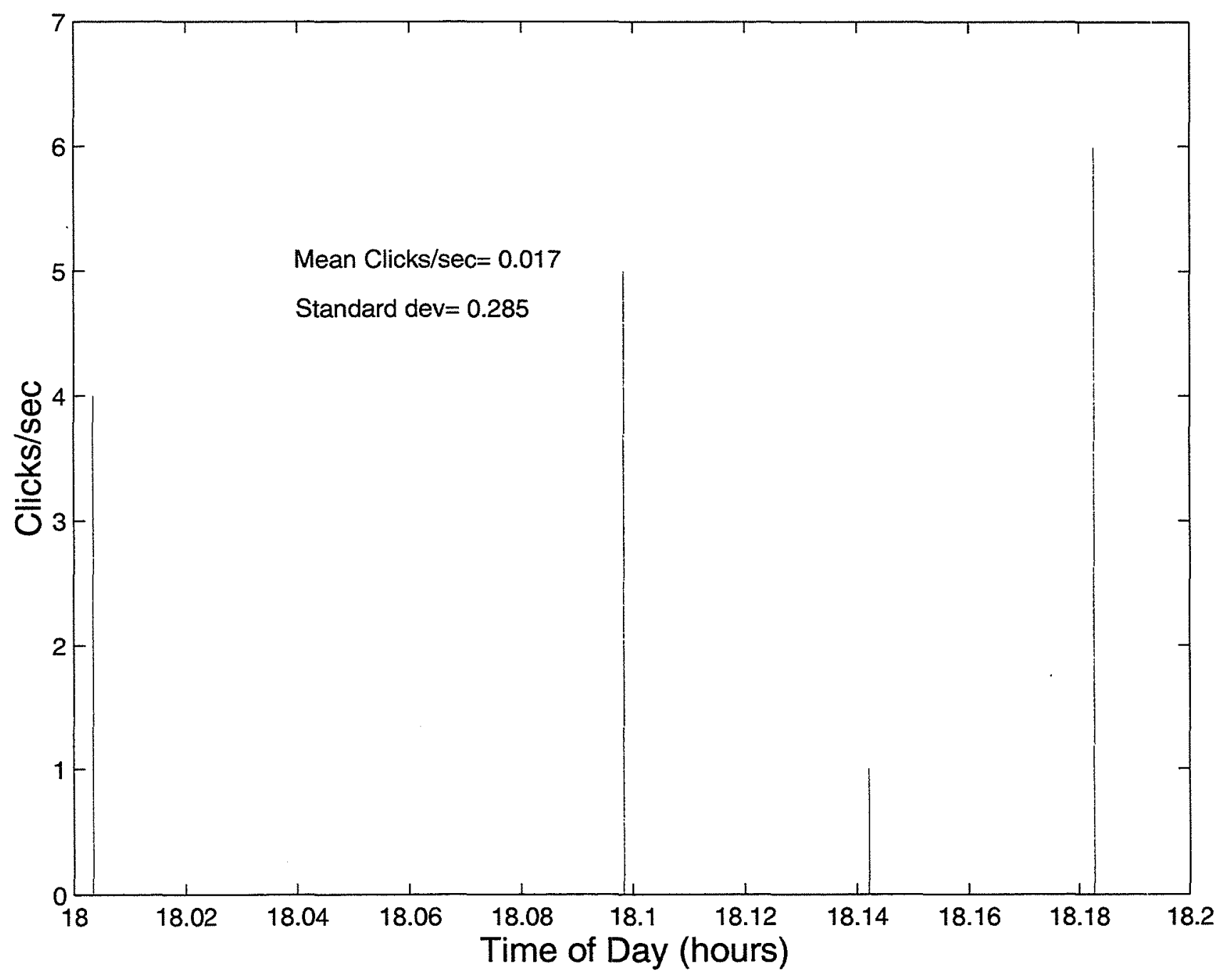

Figure 12c. Click rate record for FB09 segment 2. Mean clicks/second and standard deviation are shown in the center of the figure for the 16-minute segment as well as in the top corners for the periods before and after release. 


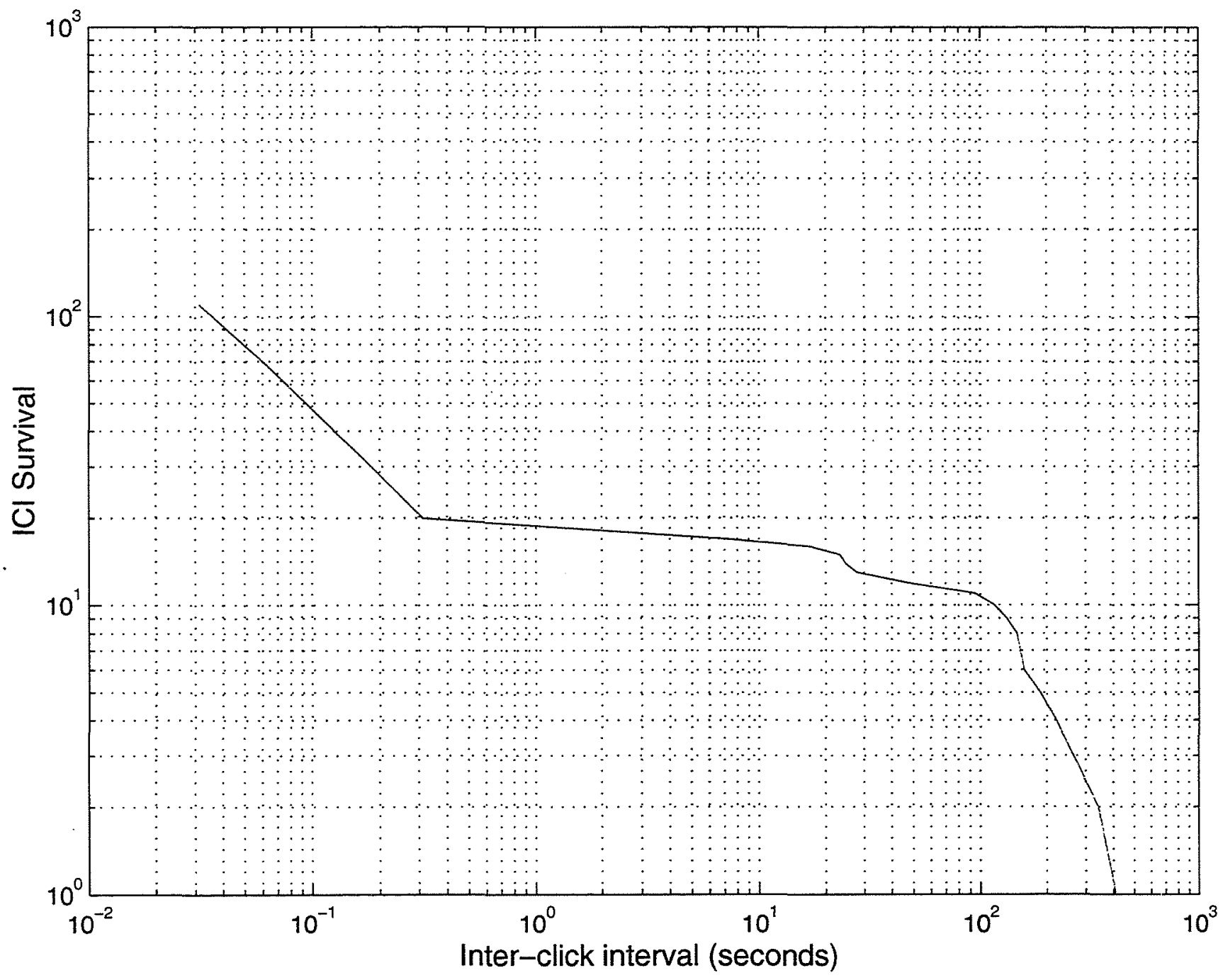

Figure 12d. Log-log survival plot of all inter-click intervals for FB09. For each ICI duration on the abscissa the value of the ordinate indicates the number of ICI's recorded that are of that duration and longer. 
Click record - F149 12Jun98 segment 1

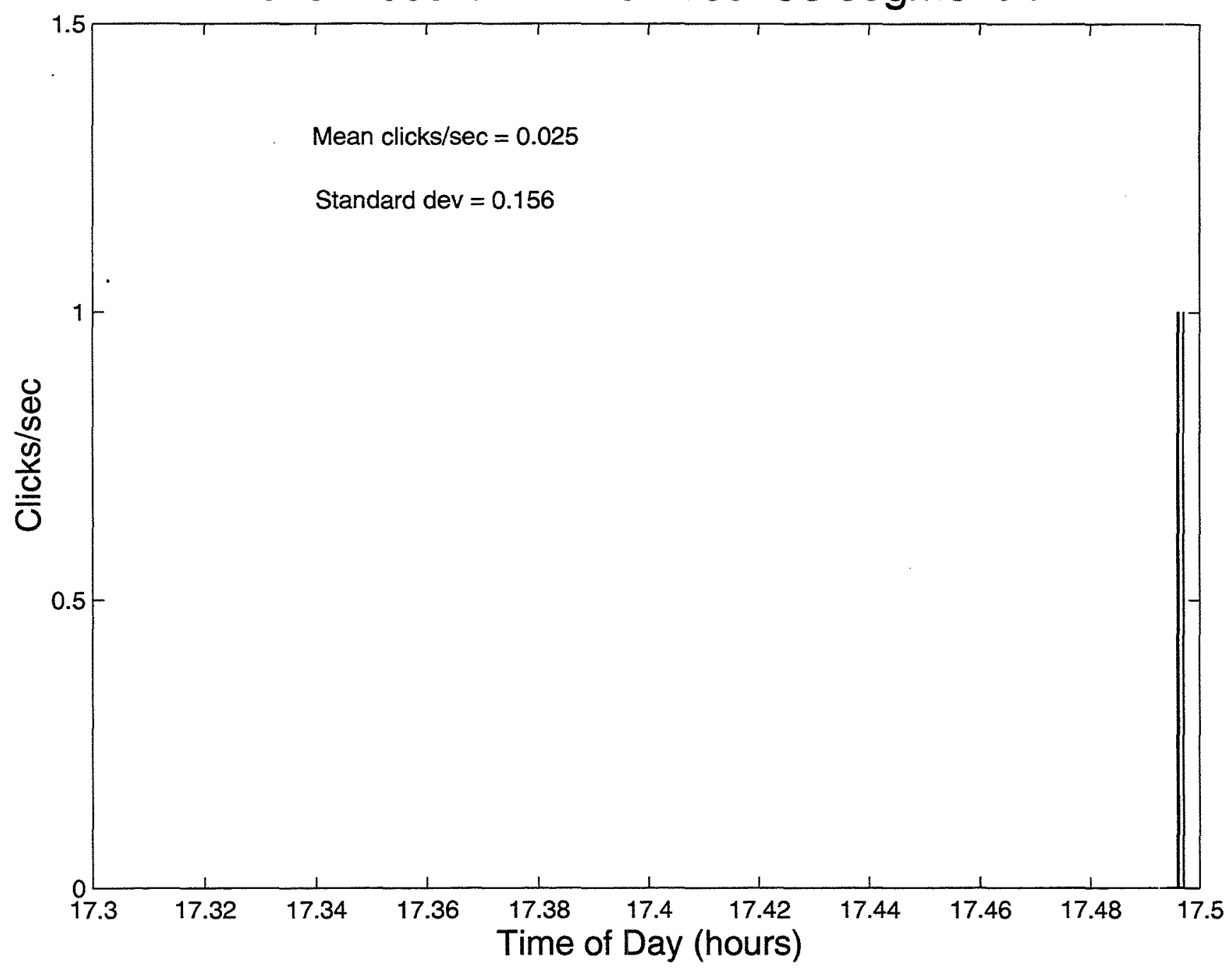

Figure 13a. Click rate record for F149 segment 1. Mean clicks/second and standard deviation are shown in the center of the figure for the 10-minute segment. Note: F149 was not sampled prior to release. 


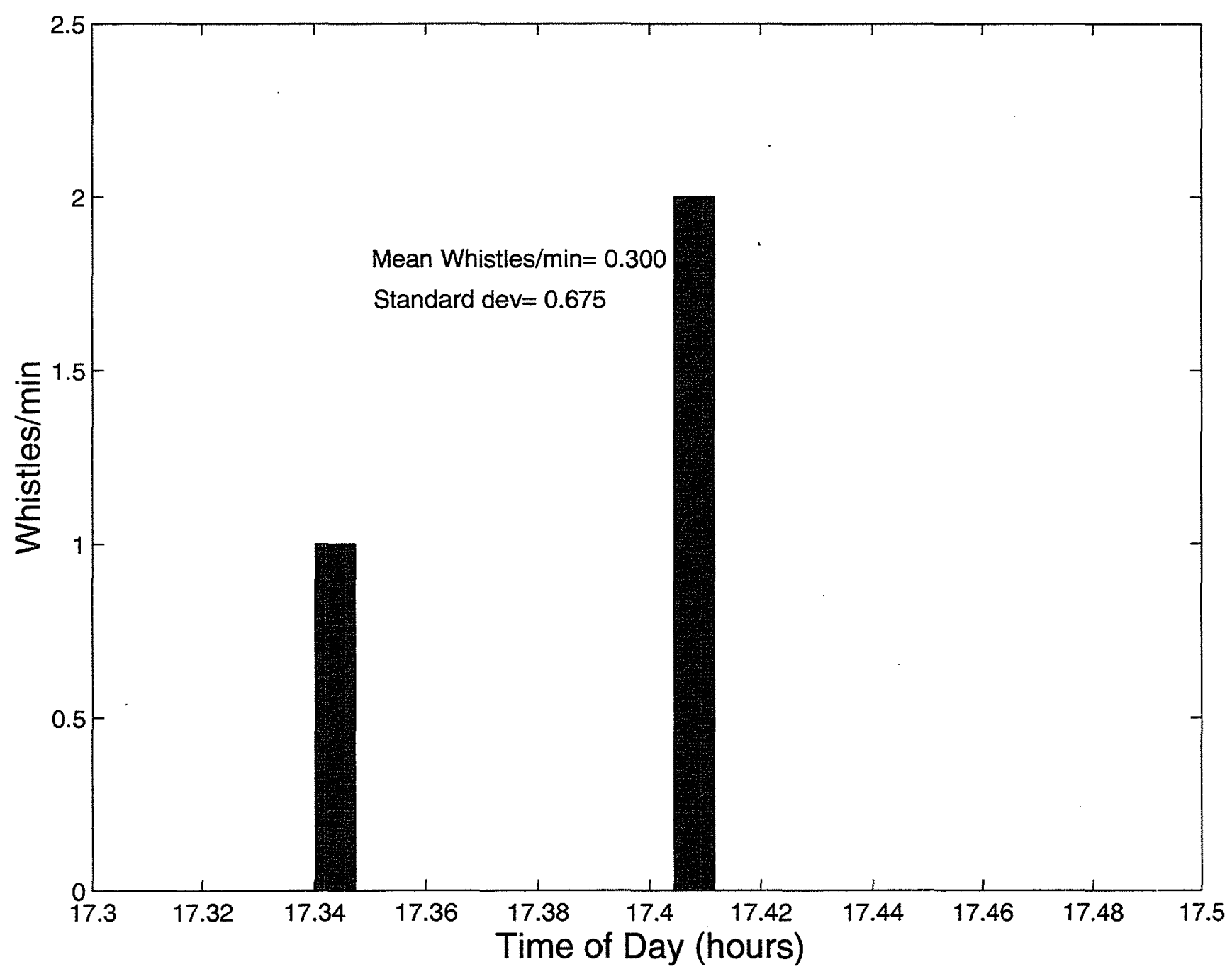

Figure 13b. Whistle rate record for F149 segment 1. Mean whistles/minute and standard deviation are shown in the center of the figure for the 10-minute segment. Note: F149 was not sampled prior to release. 


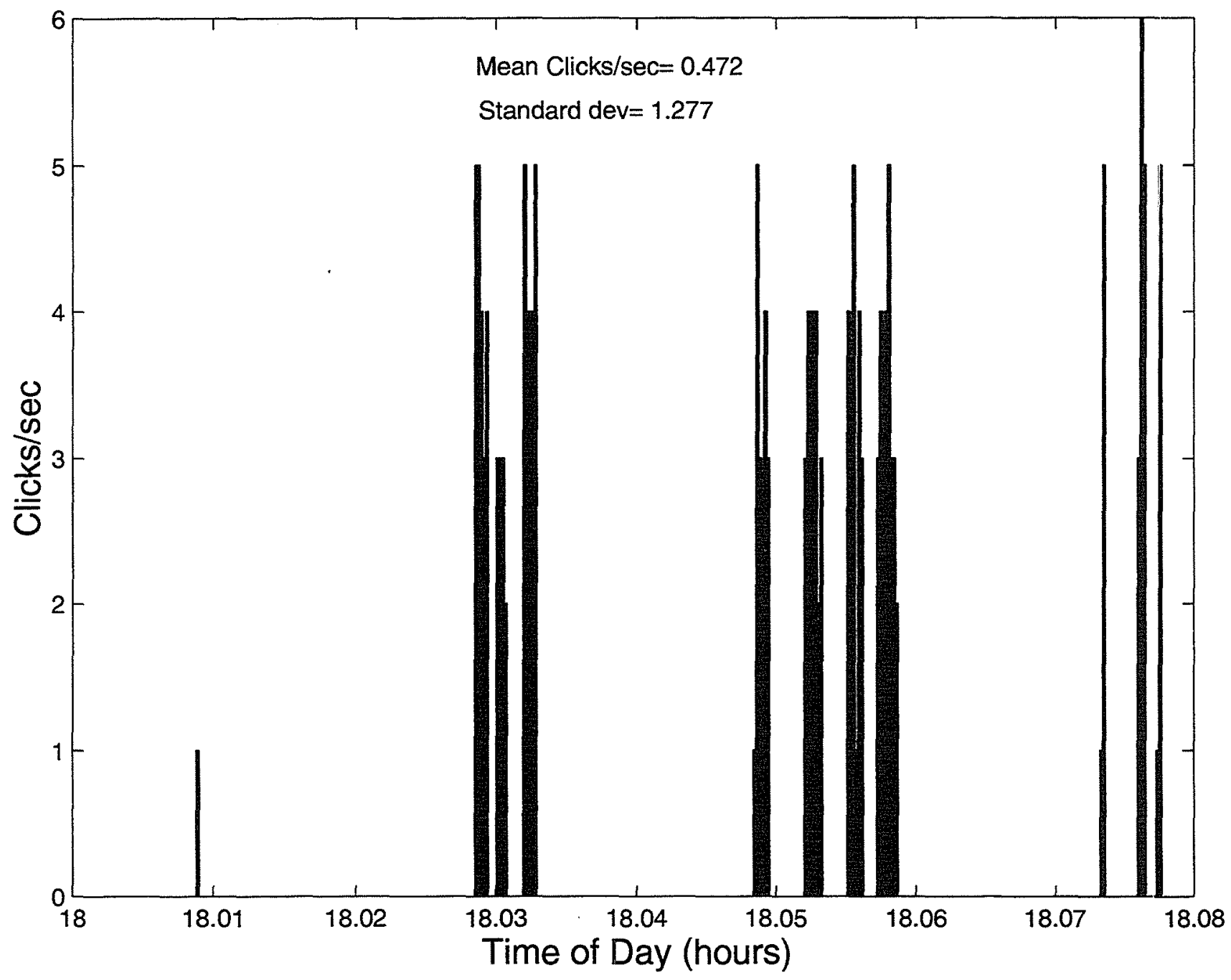

Figure 13c. Click rate record for F149 segment 2. Mean clicks/second and standard deviation are shown in the center of the figure for the 5-minute segment. 


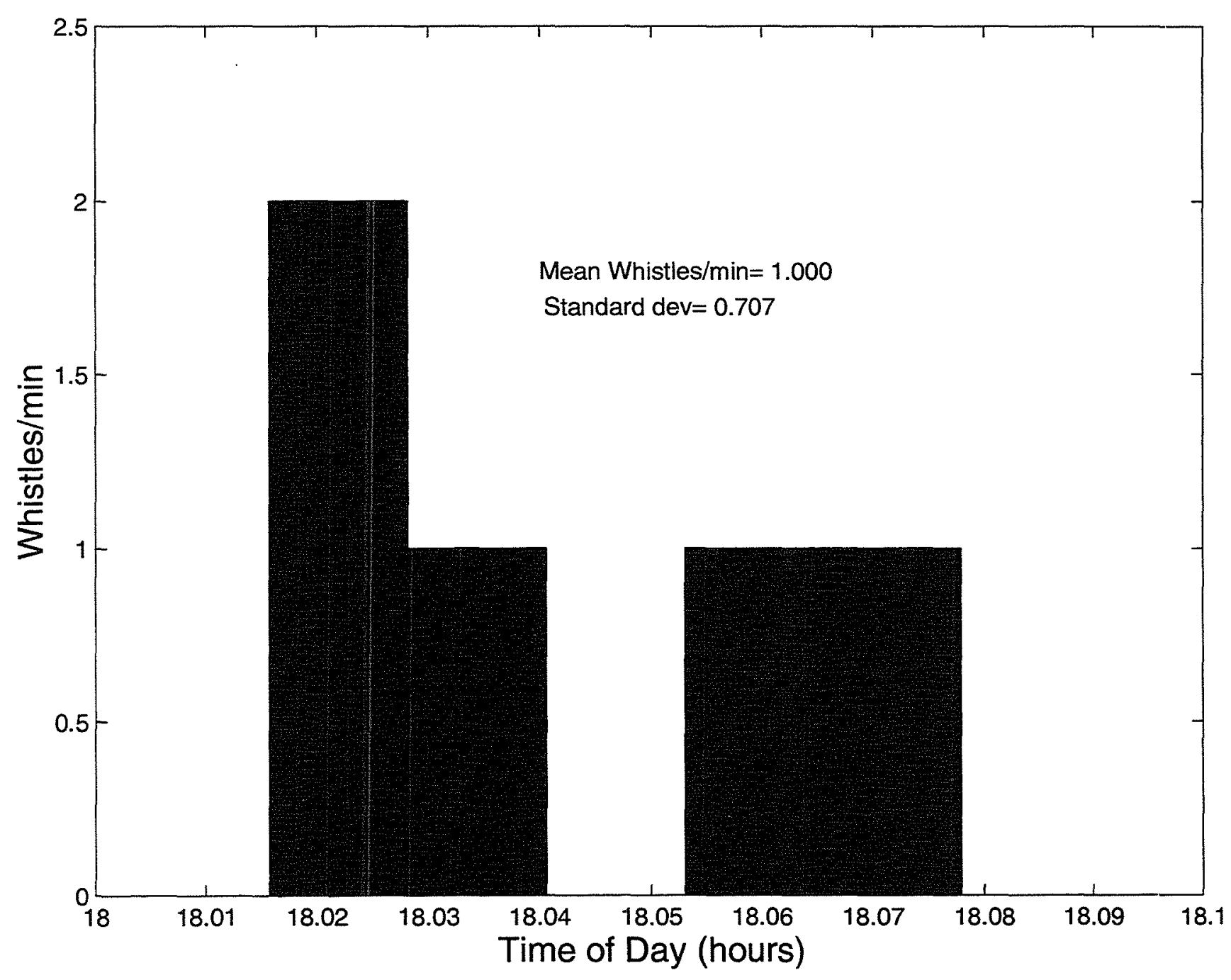

Figure 13d. Whistle rate record for F149 segment 2. Mean whistles/minute and standard deviation are shown in the center of the figure for the 5-minute segment. 


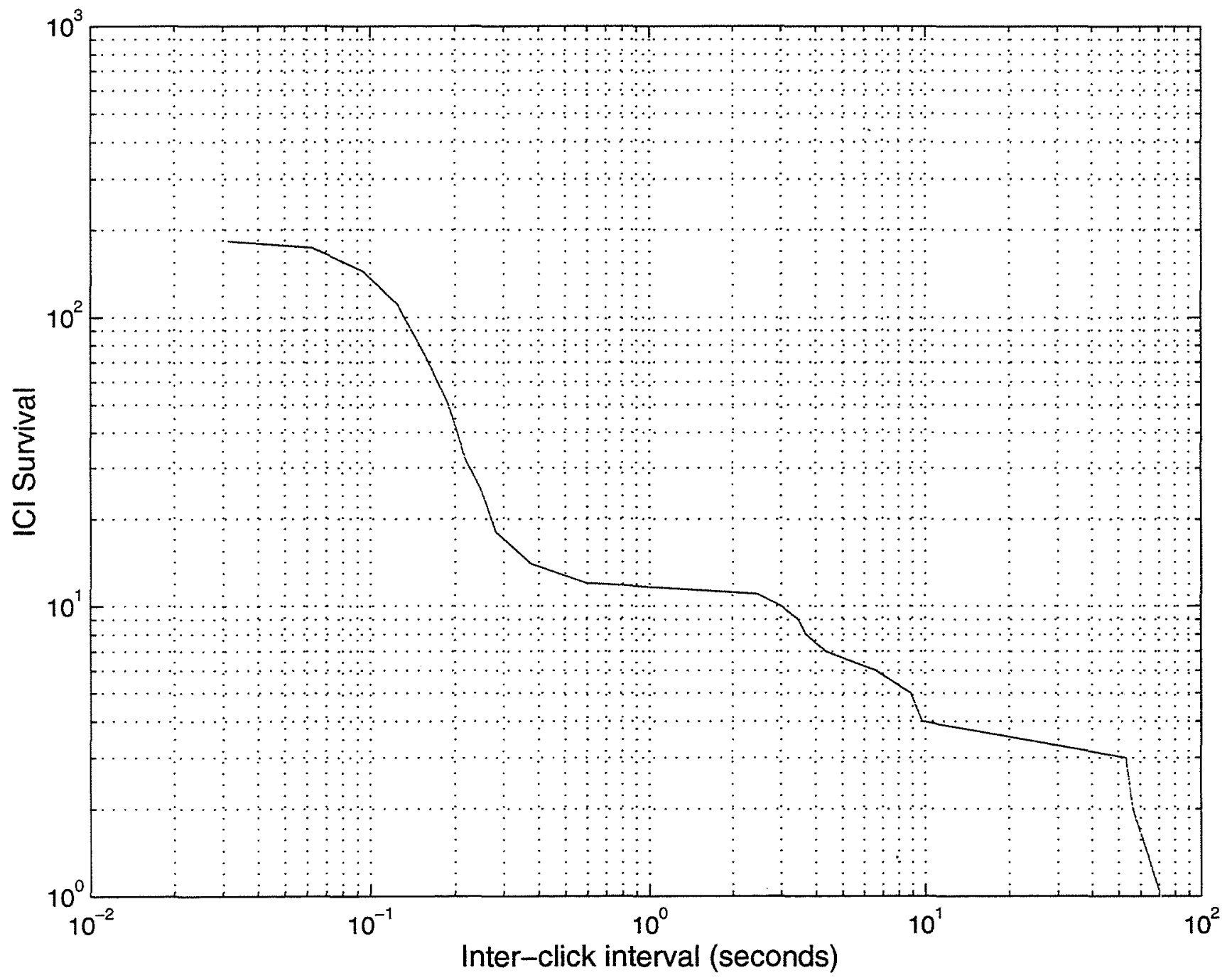

Figure 13e. Log-log survival plot of all inter-click intervals for F149. For each ICI duration on the abscissa the value of the ordinate indicates the number of ICI's recorded that are of that duration and longer. 


\subsection{Literature Cited}

Amundin, M. (1991). Sound production in odontocetes with emphasis on the harbour porpoise, Phocoena phocoena. Ph.D. thesis, University of Stockholm.

Arita, H. T. \& Fenton, M. B. (1997). Flight and echolocation in the ecology and evolution of bats. Trends in Ecology and Evolution 12: 53-58.

Astrup, J. \& Mfhl, B. (1993). Detection of intense ultrasound by the cod (Gadus morhua). Journal of Experimental Biology 182: 71-80.

Au, W. W. L. (1993). The sonar of dolphins. Springer-Verlag, New York.

Au, W. W. L., Andersen, L. N., Rasmussen, A. R., Roitblat, H. L. \& Nachtigall, P. E. (1995). Neural network modeling of a dolphin's sonar discrimination capabilities. The Journal of the Acoustical Society of America 98: 43-50.

Caldwell, D. K. \& Caldwell, M. C. (1967). Underwater sounds associated with the aggressive behavior in defense of territory by the pinfish, Lagodon rhomboides. Bulletin of the Southern California Academy of Sciences 66: 69-75.

Caldwell, M. C. \& Caldwell, D. K. (1965). Individualized whistle contours in bottlenosed dolphins (Tursiops truncatus). Nature 207: 434-435.

Cheney, D. L. \& Seyfarth, R. M. (1980). Vocal recognition in free-ranging vervet monkeys. Animal Behavior 28: 362-367.

Cheney, D. L. \& Seyfarth, R. M. (1982). Recognition of individuals within and between groups of free-ranging vervet monkeys. American Zoologist 22: 519-529.

Cheney, D. L. \& Seyfarth, R. M. (1988). Assessment of meaning and the detection of unreliable signals by vervet monkeys. Animal Behavior 36: 477-486.

Clark, C. W., Ellison, W. T. \& Beeman, K. (1986). A preliminary account of the acoustic study conducted during the 1985 spring bowhead whale, Balaena mysticetus, migration off Point Barrow, Alaska (SC/37/PS13). Report to the International Whaling Commission 36: 311-316.

Clark, C. W. \& Johnson, J. H. (1984). The sounds of the bowhead whale, Balaena mysticetus, during the spring migrations of 1979 and 1980. Canadian Journal of Zoology 62: 1436-1441.

Dawson, W. W. (1980). The cetacean eye. In Cetacean Behavior, Mechanisms and Functions (ed. L. M. Herman), pp. 53-100. John Wiley \& Sons, New York. 
Dunning, D. J., Ross, Q. E., Geoghegan, P., Reichle, J. J., Menezes, J. K. \& Watson, J. K. (1992). Alewives avoid high-frequency sound. North American Journal of Fisheries Management 12: 407-416.

Fletcher, S., Le Boeuf, B. J., Costa, D. P., Tyack, P. L. \& Blackwell, S. B. (1996). Onboard acoustic recording from diving northern elephant seals. Journal of the Acoustical Society of America 100: 2531-2539.

Gerhardt, H. C. (1992). Conducting playback experiments and interpreting their results. In Playback and Studies of Animal Communication, vol. 228 (ed. P. K. McGregor), pp. 59-77. Plenum Press, New York.

Gordon, J. \& Tyack, P. L. (1999). Sounds and cetaceans. In Marine Mammals: Biology and Conservation, In Press (ed. P. G. H. Evans and T. Raga). Plenum Press, London.

Griffin, D. R. (1958). Listening in the Dark. Yale University Press, New Haven.

Guofu, G. \& Kaiya, Z. (1992). Fiber analysis of the optic and cochlear nerves of small cetaceans. In Marine Mammal Sensory Systems (ed. J. A. Thomas, R. A. Kastelein and A. Y. Supin), pp. 39-52. Plenum Press, New York.

Holler, P. (1995). Orientation by the bat Phyllostomus discolor (Phyllostomidae) on the return flight to its resting place. Ethology 100: 72-83.

Horn, A. G. (1992). Field experiments on the perception of song types by birds. In Playback and Studies of Animal Communication, vol. 228. NATO ASI (ed. P. K. McGregor), pp. 191-200. Plenum Press, New York.

Ivanov, M. P. \& Popov, V. V. (1979). Characteristics of the dolphin's sonar emission in the detection of various types of submerged objects at limiting distances. In Marine Mammals; 7th All-Union-Conference, pp. 141-142. Center for Scientific Research Institute and Technology and Economic Studies of Fishery, Moscow, Simferopol, USSR.

Kick, S. A. \& Simmons, J. A. (1984). Automatic gain control in the bat's sonar receiver and the neuroethology of echolocation. The Journal of Neuroscience 4: 27252737.

Mann, D. A., Lu, Z. \& Popper, A. N. (1997). A clupeid fish can detect ultrasound. Nature 389: 341 . 
McGregor, P. K. (1992). Quantifying responses to playback: one, many, or composite multivariate measures? In Playback and Studies of Animal Communication, vol. 228 (ed. P. K. McGregor), pp. 79-96. Plenum Press, New York.

Miller, L. A., Pristed, J., Mfhl, B. \& Surlykke, A. (1995). The click-sounds of narwhals (Monodon monoceros) in Inglefield Bay, Northwest Greenland. Marine Mammal Science 11: 491-502.

Miller, P. J. \& Tyack, P. L. (1998). A small towed beamforming array to identify vocalizing resident killer whales (Orcinus orca) concurrent with focal behavioral observations. Deep-Sea Research II 45: 1389-1405.

Murayama, T. \& Somiya, H. (1998). Distribution of ganglion cells and object localizing ability in the retina of three cetaceans. Fisheries Science 64: 27-30.

Neuweiler, G. \& Mohres, F. P. (1966). The role of spatial memory in the orientation of

- bats. In Les Systemes Sonars Animaux Biologie et Bionique (ed. R. G. Busnel), pp. 129-140. Nato Advanced Study Institute, Frascati.

Norris, K. S., Prescott, J. H., Asa-Dorian, P. V. \& Perkins, P. (1961). An experimental demonstration of echolocation behavior in the porpoise, Tursiops truncatus (Montagu). Biological Bulletin 120: 163.

Nowacek, D. P., Tyack, P. L., Wells, R. S. \& Johnson, M. P. (1998). An onboard acoustic data logger to record biosonar of free-ranging bottlenose dolphins. Journal of the Acoustical Society of America 103: 1409-1410.

Popper, A. N. (1980). Sound emission and detection by delphinids. In Cetacean Behavior (ed. L. M. Herman), pp. 1-53. John Wiley and Sons, New York.

Schnitzler, H.-U. \& Henson, O. W. J. (1980). Performance of animal sonar systems: I. Microchiroptera. In Animal Sonar Systems (ed. R. G. Busnel and J. F. Fish), pp. 109-181. Plenum Press, New York, London.

Schnitzler, H.-U., Kalko, E. K. V., Kaipf, I. \& Grinnel, A. D. (1994). Fishing and echolocation behavior of the greater bulldog bat, Noctilio leporinus, in the field. Behavioral Ecology and Sociobiology 35: 327-345.

Searcy, W. A. (1992). Measuring responses of female birds to male song. In Playback and Studies of Animal Communication, vol. 228. NATO ASI Series (ed. P. K. McGregor), pp. 175-189. Plenum Press, New York. 
Struhsaker, T. T. (1967). Auditory communication among vervet monkeys (Cercopithecus aethiops). In Social Communication among Primates (ed. S. A. Altmann), pp. 281-324. University of Chicago Press, Chicago.

Turl, C. W. \& Penner, R. H. (1989). Differences in echolocation click patterns of the beluga (Delphinapterus leucas) and the bottlenose dolphin (Tursiops truncatus). Journal of the Acoustical Society of America 86: 497-502.

Watkins, W. A. \& Schevill, W. E. (1972). Sound source location by arrival times on a non-rigid 3-dimensional hydrophone array. Deep-Sea Research 19: 691-706.

Wood, F. G. J. (1953). Underwater sound production and concurrent behavior of captive porpoises, Tursiops truncatus and Stenella plagiodon. Bulletin of Marine Science for Gulf and Caribbean 3: 120-133. 


\section{Foraging Behavior of Sarasota Bottlenose Dolphins}

\subsection{Introduction}

In their presentation of foraging theory Stephens and Krebs (1986) develop and evaluate models of foraging behavior. These models explore the economics of foraging using detailed observations of the metabolic requirements of a predator, its movement patterns and decisions, and the energetic value of its prey. To achieve this level of analysis, these models assume that the observer can measure the energy an animal invests in foraging and the return it receives. One example of the data needed to use these models is the duration of time an animal spends searching for prey. Measuring this duration is obviously predicated on the ability to know when the animal is searching. Such knowledge results from careful, prolonged observations of entire foraging sequences, which are relatively easy to obtain for terrestrial animals (Clarke, Jones \& Jarman, 1995; Evans, 1982; Schmid-Hempel, Kacelnik \& Houston, 1985). For cetaceans, however, it has not been possible to define the behavioral components of the foraging sequence. Poorly defined and ambiguous behavioral states (e.g. feed/travel) are prevalent in the literature, largely due to the lack of a quantitative link between "feedingassociated' behaviors and actual prey capture. The current study has solved the observational problems required to define the stages of foraging in bottlenose dolphins. The data presented here unambiguously define a set of foraging behaviors and their relationship to the entire foraging sequence. This result was accomplished with a novel observational tool that permits continuous observation of dolphins and by rooting the behavioral analyses with an observed prey capture event.

The stages of foraging described by Stephens and Krebs (1986) are intuitive and straightforward, but defining and/or recognizing the behaviors that characterize these stages may or may not be. Searching, for example, occurs “... as long as no prey item or patch is detected while foraging" (Stephens \& Krebs, 1986). This definition assumes that 
the observer knows when a predator is foraging, presumably based on particular behaviors observed. Knowing whether a particular behavior qualifies as a search behavior is difficult because search behaviors can obviously occur in the absence of a successful capture. How then do we begin to recognize and define the behaviors that belong to this and later stages? Perhaps starting with a more recognizable stage would be helpful. The most easily recognized foraging stage, capture, is analogous to other 'end goal' behaviors such as copulation and fighting. And while these behaviors are not necessarily simple, their position in a sequence is unequivocal. The preceding (e.g. search) behavioral stages vary widely in their duration, constitution, and complexity which may confuse where they belong in the overall sequence. Therefore, in this study I root the analyses of feeding with confirmed capture events, and then look backward to analyze the entire sequence.

Not only is capture easy to relate to feeding, but also it is a simple, unambiguous action whose interpretation is straightforward compared to other behaviors. Prey capture, copulation, and fighting all fit into the category of 'consummatory actions' as defined originally by Craig (1918) and given context and insight by Tinbergen (1951). Tinbergen (1951) explains these consummatory actions:

"The consummatory act is relatively simple; at its most complex, it is a chain of reactions, each of which may be a simultaneous combination of a taxis and a fixed pattern." p. 106

Tinbergen (1951) also discusses the occurrence of non-consummatory behaviors, which appear to be 'exploratory' or 'seeking' behaviors. These behaviors are not characterized by stereotyped motor patterns but rather are variable, plastic, and purposeful. Craig (1918) also recognized this class of behaviors as well and termed them as 'appetitive' to convey the fact that the animal is striving to achieve some end goal. Tinbergen's (1951) description of these behaviors gives insight into the difficulty in studying them: 
"But appetitive behavior is a true purposive activity, offering all the problems of plasticity, adaptiveness, and of complex integration that baffle the scientist in his study of behaviour as a whole. Appetitive behaviour is a conglomerate of many elements of very different order, of reflexes, of simple patterns like locomotion, of conditioned reactions, of 'insight' behaviour, and so on. As a result it is a true challenge to objective science, and therefore the discrimination between appetitive behaviour and consummatory act is but a first step of our analysis." p. 106

The purposive end of appetitive behavior is then the consummatory action, but, as Tinbergen (1951) discusses, an appetitive behavior by no means always leads directly to the performance of a consummatory act. In the case of foraging, searching, detection, decision, and pursuit serve the purpose of capturing and consuming prey. However, the appetitive behaviors may occur without leading directly to prey capture. The choice of which appetitive behaviors are displayed can be influenced by a variety of stimuli. Tinbergen (1951) describes an example that serves very well to illustrate this point:

"...the hunting of a peregrine falcon usually begins with relatively random roaming around its hunting territory, visiting and exploring many different places miles apart. This first phase of appetitive behaviour may lead to different ways of catching prey, each dependent on special stimulation by a potential prey. It is continued until such a special situation is found: a flock of teal executing flight manoeuvres, a sick gull swimming apart from the flock, or even a running mouse. Each of these situations may cause the falcon to abandon its 'random' searching. But what follows then is not yet a consummatory action, but appetitive behaviour of a new, more specialized and more restricted kind. The flock of teal releases a series of sham attacks serving to isolate one or a few individuals from the main body of the flock. Only after this is achieved is the final swoop released, followed by capturing, killing, plucking, and eating, which is a relatively simple and stereotyped chain of consummatory acts. The sick gull may provoke the release of sham attacks tending to force it to fly up; if this fails the falcon may deftly pick it up from the water surface. A small mammal may release simple straightforward approach and subsequent capturing, etc." p. 106-107

This example demonstrates the plasticity and adaptiveness of appetitive behaviors and the gradual narrowing as they all lead to the consummatory prey capture and consumption. 
Individual variation can also account for differences in appetitive foraging behaviors. Intraspecific foraging specialization is common (Goss-Custard \& Le V dit Durell, 1983; Goss-Custard \& Sutherland, 1984; Hoelzel, Dorsey \& Stern, 1989; Kalcounis \& Brigham, 1995; Kodha, 1994; McLaughlin, Grant \& Kramer, 1992; Nakamichi et al., 1998; Rogers \& Bryden, 1995; Swennen et al., 1983) and can persist through generations (Norton-Griffiths, 1967). Why and how are intraspecific differences in foraging strategies evolutionarily stable? Game theory has been used effectively to explore the 'why', and social-psychological theory has addressed the 'how' or the transmission of behavioral traits. Maynard-Smith (1982) discusses the primary game theory hypothesis that could explain their existence. Partridge and Green (1985) use this and other game theory ideas as the basis for three specific mechanisms by which individually specific foraging strategies could evolve and persist: 1) Food patches may be distributed such that unique strategies maximize exploitation; 2) Morphological or phenotypic variation, e.g. age or sex class, could predispose different individuals to specific foraging techniques; 3 ) The success of one strategy may depend on how many conspecifics in the population utilize it, i.e. if all animals in the population use the same strategy, then the resource it exploits could become over-utilized. This hypothesis assumes identical phenotypes or multiple phenotypes at equilibrium within the population and predicts a distribution of animals among foraging strategies that are energetically equivalent when the distribution is dictated by frequency-dependent selection (Maynard Smith, 1982).

Several traits associated with the foraging ecology of the oystercatcher, Haematopus ostralegus, demonstrate the stability, potential benefits, and the combination of forces shaping individually specific foraging strategies within a population. Oystercatchers specialize both in prey type, mussels vs. worms, and in the method they use to open mussels, Mytilus edulis. As an example of the second mechanism listed above, oystercatchers develop morphological specialization for opening mussels. Distinct bill forms are associated with each feeding strategy (Goss-Custard \& Le V dit 
Durell, 1983; Norton-Griffiths, 1967; Swennen et al., 1983). Swennen et al. (1983) showed that with changing prey fields, individual oystercatchers altered their foraging strategy. The perpetually growing bill was shaped by the different forces required by the specific technique (e.g. drilling, hammering, or chiseling) and eventually changed to the appropriate form for that technique. Longitudinal studies have shown that young oystercatchers initially learn their foraging technique from their parents (Goss-Custard \& Le V dit Durell, 1983; Norton-Griffiths, 1967; Swennen et al., 1983). Individual birds, however, change strategies as they age (i.e. mechanism \#2) (Goss-Custard \& Le V dit Durell, 1983) and with changing prey availability (i.e. mechanism \#1) (Swennen et al., 1983). Despite this plasticity each bird does appear to favor a particular technique where the frequency of the different techniques could be produced by frequency-dependent selection or mechanism \#3 (Goss-Custard, Le V dit Durell \& Ens, 1982; Goss-Custard \& Sutherland, 1984). The oystercatcher system appears to be the result of a combination of all three mechanisms described above: 1) specialized prey patches; 2) phenotypic differences (although there is plasticity here too); and 3) intraspecific competition or frequency dependence. At least two highly desirable benefits appear to be conferred by this flexible system. Such plasticity in an individual's foraging ecology leaves it better able to adapt to rapid environmental changes. In addition, the highly specialized feeding strategies reduce indirect competition caused by resource depletion (Sutherland, 1987) as the oystercatchers switch from worms to mussels or even specialize within the mussel prey (Le V. dit Durell \& Goss-Custard, 1984).

Other species exhibit individual foraging specialization and/or variation (Beauchamp, Giraldeau \& Ennis, 1997; Kalko, 1995; Kodha, 1994; McLaughlin et al., 1992; Nakamichi et al., 1998) with differences as basic as sexual dimorphism affecting strategies available to individuals (Kalcounis \& Brigham, 1995). Marine mammals also demonstrate the capacity for intraspecific foraging specialization (Rogers \& Bryden, 1995). Most examples of cetaceans, however, are based on stomach contents rather than 
behavior (Young \& Cockcroft, 1994), or have some difficulty demonstrating that strategies are significantly distinct (Hoelzel et al., 1989).

\subsection{Materials and Methods}

\subsubsection{Field Operations}

Two techniques were used to collect behavioral data: focal-animal and instantaneous sampling sensu Altmann (1974). Continuous focal-animal behavioral data and ambient acoustic data were collected with the integrated observation platform developed by Nowacek (1995)(also see Appendix 1). Briefly, this platform consisted of a video camera suspended from a helium-filled aerostat tethered to an observation vessel (OV) and two towed hydrophones deployed from the vessel as described by Sayigh et al. (1993). The aerostat was flown at approximately $50 \mathrm{~m}$ altitude. From the $6 \mathrm{~m}$, outboard powered, partially enclosed OV the camera was controlled with a $360^{\circ}$ continuous pan (max speed $100^{\circ}$ per second), $90^{\circ}$ tilt, and iris and focus control. The incoming audio and video signals were monitored continuously and adjusted to obtain optimum recordings (see Figure A1.1). Animals were followed from the $\mathrm{OV}$ at a distance of $>=15 \mathrm{~m}$. The overhead video often provided continuous footage of dolphins throughout the water column, although in deeper waters $(>2 \mathrm{~m})$ the animals were sometimes not visible. The two hydrophones were modified to reduce flow noise, and the acoustic signal was usually filtered through a $4 \mathrm{kHz}$ high-pass filter (Allen Avionics 4188-4PO). Video and audio signals were recorded on a single deck, initially a Sony BetaCam, then $\mathrm{Hi}-8$, and currently on a digital video recorder (Sony HR1000).

Each day the study area (Figure 1.1) was searched until potential focal animals were located. Dependent calves (i.e. those always sighted with their mothers) were excluded from the study for two reasons. Their feeding rate was presumably lower than independent animals as they were still nursing, and their foraging patterns were still developing (Caro \& Hauser, 1992; Haenel, 1986; Lopez \& Lopez, 1985) which could 
give a biased view of behaviors observed. Once animals were located, a focal follow was begun if one of the animals present was considered to be appropriate, i.e. not a dependent calf nor a subject that had been observed for $\geq 5$ hours, and after basic census and photoidentification data were collected. The sample of individuals studied represents a cross-section of sub-adult and adult animals (Table 4.1). While following a focal animal, the camera operator attempted to maintain the best possible image by adjusting the focus and iris and keeping the individual in the field of view with as much zoom as possible, i.e. extent of zoom was subject to movement of the animal and the aerostat. Data from the video footage were scored in the laboratory (Section 4.2.2). At least two other observers and a boat operator participated in the follow. The other observers recorded instantaneous samples at 5-minute (1996) or 3- and 21-minute (1997 \& 1998) intervals; the change in sampling protocol was made due to an undersampling of some behavioral data and oversampling of other data (e.g. tides, weather conditions). Observers also kept a 'capture event record' which logged every observed prey capture attempt, successful or not, seen from either vantage point. Behavioral data were sampled at 3-minute interval times and environmental data were sampled at 21-minute sample intervals. If the focal individual was not visible at the time point, either the video operator or direct observer collected data at the next confirmed observation of the individual. Instantaneous samples and the capture record were entered into a spreadsheet. Focal follows were terminated due to observer fatigue ( $\geq 2$ hrs.), losing contact with the animal, or inclement weather.

\subsubsection{Scoring Behavior from Videotapes}

Focal animal data were scored from videotapes in real-time by entering the data into an observational data computer program (The Observer ${ }^{\circledR}$, v3.0). The periods of time during which the focal individual was not visible on the video tape ('time-outs') were counted using the Observer's ${ }^{\text {(B) }}$ suspend/resume function. Scoring was suspended if detailed behavior could not be collected for 10 seconds (i.e. a progressive loss of detail sometimes occurred as an animal gradually disappeared from view due to depth, glare, etc.) and resumed when the animal was continuously visible. Altmann (1974) notes that 
even if an animal is not continuously visible, rates and frequencies of behaviors can be accurately measured provided the time-outs are logged. Occasionally other animals were also continuously visible in the video footage. Mann (1999) states that conducting separate 'video' follows of different individuals captured on the same tape is equivalent to multiple focal-individual follows provided simultaneous behaviors are accounted for in statistical analyses. Thus, when additional animal(s) fit the sampling criteria and could be sampled as focal individuals these follows were also scored; instantaneous environmental samples for the initial focal were utilized. Based on the configuration of The Observer $®$, multiple classes of behaviors can be simultaneously scored with states being mutually exclusive within a class. The behavioral states and events defined specifically for this study were all included in one class with all states being mutually exclusive (Section 4.3.2). Other classes of behavior scored were: 1) general activity categories (i.e. travel, social, mill, probable feed, feed, rest, (Urian \& Wells, 1996); 2) presence/absence of a dependent calf; and 3) the distance between the focal animal and its nearest neighbor. With this configuration, the behaviors of interest in the current study could be investigated in relation to the other three classes. For example, the focal's general activity or the distance to its nearest neighbor could be analyzed currently with a search behavior or prey capture. After being scored in the computer program the data were tabulated and exported for statistical analyses.

The study area was divided into 10 different habitats, nine of which were defined by Waples (1995). An additional habitat was added in this study after preliminary observations from the overhead video. In addition to 'seagrass' and 'sand', 'seagrass edge' was added. The dolphins not only swam within and through the seagrass beds, but also actively maintained themselves within approximately 1 body length of the edge of the seagrass bed while swimming. Without the overhead perspective, this could easily be scored as time within the grass beds, but the animals were more precise in their orientation than simply being 'in' or 'out' of the seagrass meadows. The habitat in which 
a particular behavior occurred was scored in The Observer $($ as a modifier. For analyses, the behaviors could be sorted by modifier or across all modifiers.

\subsubsection{Analysis of Behavioral Sequences}

As discussed above, using the capture to root the analyses provides an unambiguous point from which the entire sequence can be studied. Appetitive behaviors presumably occur in the absence of a successful capture, so their frequency may be greater than that of the consummatory behavior. Dolphins may search for but fail to find prey; they may successfully search for and detect prey but decide not to pursue; or they may decide to pursue but fail to successfully capture the prey item. Handling behaviors are not good markers of prey capture as they may or may not occur depending on prey type and size.

From the list of behaviors scored specifically for this study (Section 4.3.2), a method was needed to investigate the significance of a given behavior in the foraging sequence. Assuming that behaviors from earlier in the foraging sequence occur more frequently than successful captures, this method must be sensitive to the actual relationships between behaviors and not the raw frequency of occurrence, i.e. the significance of early, frequent behaviors could be overestimated at the expense of later, infrequent but essential behaviors. Conditional probabilities represent a sound method of measuring these relationships because they consider not only the raw frequency of a behavior but also the dependence of later behaviors on earlier ones. One test that uses conditional probabilities to gauge the sequential dependence of the latter behavior or 'target' on the preceding behavior or 'given' is the $z$-score binomial test (Bakeman $\&$ Gottman, 1986). A $z$-score is calculated using the conditional probability of the occurrence of the 'target' after the 'given' occurs. The following equation shows the calculation of a $z$-score: 


$$
z=\frac{P(t / g)-P(t)}{\sqrt{\frac{P(t)[1-P(t)]}{N P(g)}}} P(t)=\frac{f(t)}{N}
$$

$\mathrm{N}=$ total number of paired behavioral transitions tallied; $\mathrm{P}(\mathrm{t} / \mathrm{g})=$ probability of the 'target' occurring given the occurrence of the 'given' behavior; $\mathrm{P}(\mathrm{t})=$ probability of the 'target'; $\mathrm{P}(\mathrm{g})=$ probability of the 'given'.

Bakeman and Gottman (1986) state that as $\mathrm{N}$ increases beyond 25 and $\mathrm{N} * \mathrm{P}(\mathrm{t} / \mathrm{g})\{1-\mathrm{P}(\mathrm{t} / \mathrm{g})\}>9$, the binomial distribution approximates a normal distribution. If these conditions hold then if $z> \pm 1.96$ the observed probabilities are significantly different from expected at the 0.05 level. Bakeman and Gottman (1986) also discuss the issue of independence in the context of successive events and $z$-scores. They assert that because dyadic states in successive time intervals are likely not independent it is most conservative to consider the $z$ simply as an index or score and not to assign $p$-values to it. In the present study, however, the samples should be considered independent for two reasons. First, because only a single animal's behavior is examined rather than a dyadic interaction. Secondly, as can be inferred from Anderson and Goodman (1957), the assumption of independence in this case refers to the lack of sequential structure in the null model.

$Z$-scores and first order Markov models can be calculated for behavioral transitions in an entire multi-behavioral sequence. In this study a single-step transition analysis was chosen to investigate the interdependence of foraging behaviors one step at a time and to facilitate inclusion of all potential appetitive behaviors. The analysis proceeded in a 'backwards' fashion in that significant relationships between two behaviors were investigated in reverse chronological order beginning with the terminal event of prey capture. State-lags consider only the behavior immediately preceding the target whereas time lags consider all behaviors that occurred during a specific time period 
prior to the target event. $Z$-scores were calculated for up to four state-steps before capture, and for the three most common habitats: shallow sand, seagrass meadow, and seagrass edge.

\subsubsection{Analysis of Individual/Habitat Specific Use of Foraging Behaviors}

The foraging behavior an individual dolphin chooses to utilize at a given time can be affected by at least three factors: individual preference, habitat, and distribution and behavior of the prey. To test for influences of the first two factors, multiple contingency tables were condensed over one variable and tested against the other (e.g. foraging behaviors of a single animal across all habitats). In addition, the occurrences of each behavior/habitat combination across all individuals were tested against that behavior in the other habitats and against all other behaviors in a similar habitat breakdown (Section 4.3.4). Condensing contingency tables reduces the degrees of freedom for each analysis which increases the power of the test (Everitt, 1977). From these condensed tables relatively specific questions about the influences of individual preference and habitat can be addressed. For large contingency tables (e.g. Table 4.4) a more expeditious means of identifying categories responsible for a significant chi-square value is to examine adjusted residuals (Everitt, 1977). Adjusted residuals are calculated as follows:

First the standardized residual is calculated:

$$
e_{i j}=\left(n_{i j}-E_{i j}\right) / E_{i j}
$$

where $\mathrm{n}_{\mathrm{ij}}$ is the observed value in row $i$ column $j$ and $\mathrm{E}_{\mathrm{ij}}$ is the expected value for that observation calculated as $n_{i} \bullet{ }^{*} n_{\bullet j} / N$ where $N$ is the total number of observations for the entire table.

Next an estimate of the variance of the standardized residual, $\mathrm{e}_{\mathrm{ij}}$, is calculated:

$$
v_{i j}=\left(1-n_{i \bullet} / N\right) *\left(1-n_{\bullet j} / N\right)
$$

Finally, the adjusted residual for a cell $i j$ in the contingency table is computed using: 


$$
d_{i j}=e_{i j} / \sqrt{ } v_{i j}
$$

The adjusted residuals can then be compared to the standard normal deviate (i.e. 1.96) to obtain a level of significance (i.e. P-value) for a specific cell in the contingency table (Everitt, 1977). For example, $\left|\mathrm{d}_{\mathrm{ij}}\right| \geq 1.96$ can be considered significant at the $P<0.05$ level. Further, $\left|d_{i j}\right| \geq 9.76$ is equivalent to $P<0.01$ and $\left|d_{i j}\right| \geq 96$ is equivalent to $\mathrm{P}<0.001$. In addition, residuals can be significantly positive or negative, identifying results that are significantly more or less common than expected, respectively.

\subsection{Results}

\subsubsection{Focal animals: profiles and data collected}

Data were collected over a total of 7.5 months during May-September of three years (1996-98). Table 4.1 lists the focal animals and summarizes data collection by individual for the entire study. Figures 4.1 a and b show the focal animals' habitat use. 


\begin{tabular}{|c|c|c|c|c|c|}
\hline \multirow{2}{*}{$\begin{array}{l}\text { Focal } \\
\text { Animal }\end{array}$} & \multirow{2}{*}{$\begin{array}{l}\text { Birth } \\
\text { Year }\end{array}$} & \multirow{2}{*}{ Sex } & \multirow{2}{*}{$\begin{array}{c}\text { Reproductive } \\
\text { Status }\end{array}$} & \multicolumn{2}{|c|}{ Animal-Minutes Observed } \\
\hline & & & & Total & Video \\
\hline FB03 & 1989 & $\mathrm{~F}$ & A & 284 & 84 \\
\hline FB11 & 1984 & $\mathrm{~F}$ & A & 110 & 44 \\
\hline FB28 & 1965 & M & A & 196 & 88 \\
\hline FB38 & 1974 & M & A & 120 & 31 \\
\hline FB54 & 1971 & $\mathrm{~F}$ & A & 105 & 47 \\
\hline FB65 & 1983 & $\mathrm{~F}$ & A & 230 & 34 \\
\hline FB75 & 1974 & $\mathrm{~F}$ & A & 120 & 65 \\
\hline FB79 & 1979 & $\mathrm{~F}$ & A & 85 & 33 \\
\hline FB83 & 1950 & $\mathrm{~F}$ & A & 110 & 41 \\
\hline FB92 & 1988 & M & SA & 125 & 114 \\
\hline F101 & 1990 & $\mathrm{~F}$ & A & 115 & 31 \\
\hline F118 & 1992 & $M$ & $\mathrm{SA}$ & 185 & 138 \\
\hline F138 & 1992 & $\mathrm{M}$ & $\mathrm{SA}$ & 135 & 44 \\
\hline F155 & 1990 & $\bar{F}$ & SA & 175 & 93 \\
\hline F157 & $?$ & $\mathrm{~F}$ & A & 265 & 87 \\
\hline UNK2 & $?$ & $?$ & $\mathrm{SA} / \mathrm{A}$ & 115 & 31 \\
\hline UNK3 & $?$ & $?$ & SA/A & 100 & 36 \\
\hline Non-30's & $\mathrm{n} / \mathrm{a}$ & $\mathrm{n} / \mathrm{a}$ & $\mathrm{n} / \mathrm{a}$ & 879 & 255 \\
\hline TOTALS & & $10 \mathrm{~F}, 5 \mathrm{M}$ & $4 \mathrm{SA}, 11 \mathrm{~A}$ & 3454 & 1296 \\
\hline
\end{tabular}

Table 4.1Focal animals included in the study. UNK2\&3 were unknown at the time of publication. 'Non-30's' refers to data collected on animals for whom $<30 \mathrm{~min}$ of useable video data were collected. $\mathrm{A}=$ Adult, $\mathrm{SA}=$ Sub-adult.

\subsubsection{Definitions and Descriptions of Observed Behaviors: States (S) and Events (E)}

Rooting/Drifting (S)

In other locations, bottlenose dolphins have been observed to feed near or in the benthos (Rossbach and Herzing 1997). In these areas, dolphins swim near the bottom scanning the sediment and then stop, orient themselves vertically, and dig into the 
sediment sometimes up to their pectoral fins in search of prey (Rossbach \& Herzing, 1997). The dolphins in Sarasota Bay utilized a similar behavior, but only dug in the sediment with their rostra. This 'rooting' behavior was characterized by an animal orienting itself vertically or almost vertically in the water with its rostrum very near or in the sediment or sea grass (Figure 4.2). 'Drifting' or bottom inspection was a variation of rooting typified by the same body posture but the dolphin was not stationary; instead it maintained the described posture while moving or drifting slowly over the bottom. A rooting or drifting individual often actively maintained its posture and/or position by moving its flukes and pectoral fins. This behavior has not previously been reported in Sarasota, likely because it occurs at or near the bottom, and is rarely visible without the aid of the overhead view. For example, Shane (1990) may have seen rooting when she reported 'subsurface feeding' by dolphins near Sanibel Island, FL that made tail-stock or fluke-out dives and stirred up mud. Many dolphins in Sarasota were observed rooting in and around crab traps. These animals may have been scavenging the crab bait, hunting fish that came to scavenge, feeding on invertebrates that grow on the traps, or simply playing with the apparatus or trapped crabs as was observed on one occasion. Rooting near crab traps was never observed to directly result in a fish capture (occasional entanglement in float lines extract a cost for this pattern; R. Wells, pers. comm.).

\section{$\underline{\text { Kerplunking (E) }}$}

Kerplunking occurred when an individual raised its tail flukes out of the water, then forcefully brought its flukes downward into the water. The movement of the flukes continued with force well into the water column, i.e. the downward motion did not stop when the flukes contacted the water (Figure 4.3). Products of the kerplunk included a 1-2 m splash, a sub-surface bubble cloud and trail, and sound associated with both the event itself and the resulting bubbles. Attempts to record the sound of the actual kerplunk were unsuccessful despite the use of low frequency hydrophones close $(<10 \mathrm{~m})$ to kerplunking individuals. Kerplunking could flush fish from their refuges or cause enough movement to be detected by a foraging dolphin. One or more of the following products of a kerplunk could be used by a foraging dolphin to flush or corral fish: the impulsive low 
frequency sound pressure wave of the event, the resulting particle motion (Fay, 1988), or bubbles (Sharpe \& Dill, 1997). Kerplunking occurred most frequently when an individual was at the edge of a seagrass bed. Single as well as multiple animals were observed kerplunking, and two animals sometimes kerplunked synchronously. Connor et al. (in prep) have observed dolphins kerplunking in Shark Bay, W. Australia, and have a similar interpretation of its function.

\section{Fish whacking (E)}

Wells et al. (1987) first described fish whacking, and Shane (1990) also observed the behavior in dolphins near Sanibel. The overhead video gave a new perspective on this behavior, facilitating observation of the entire sequence. Most commonly a dolphin swam through a grass bed, turned and swam on its side, and with a forceful, fast $(<1 \mathrm{sec})$ dorsal or ventral thrust of its flukes struck a fish (Figure 4.4). The fluke thrust occurred at or near the surface, which the dolphin apparently used as a barrier, and often the fish was propelled out of the water. The fish have been observed to fly as far as $6 \mathrm{~m}$ through the air, after which the dolphin swam to the landing spot and consumed the fish. The dolphin caught its prey even when the fish did not leave the water. Propelling the fish into the air may be a by-product of the shallow water in which this behavior always occurred. In addition, using the water's surface as a barrier further limits the prey's possibility for escape. Chasing a prey item in a three-dimensional space presents a challenge, as a dolphin has a relatively small area, i.e. the mouth, with which to capture a fish. Bats have solved a similar problem by using their tail membrane to increase the surface area used to capture prey. Bats scoop insect prey into the membrane and then reach back and grasp the prey with their mouths (Simmons, Fenton \& O'Farrell, 1979). By utilizing their relatively large tail flukes (Mean=61 cm. R.Wells, pers. comm.) dolphins similarly increase the surface area with which to contact the fish, and their strong axial musculature allows them to stun or injure a fish so that it can be more easily captured. In addition, in shallow water a dolphin's flukes could occupy a significant portion of the water column leaving little space for fish to escape. This behavior could be a mechanism used after a missed capture attempt using the mouth, but none of the 
observed fish whacks occurred at the end of a chase sequence. Instead, fish whacks occurred as the dolphin(s) swam very near $(<1 \mathrm{~m})$ a school of fish, which suggests that it functions to stun or disorient one or many fish. Fish whacking always occurred in groups of $>1$ dolphin, and the whacks were often coordinated with 2-3 being produced simultaneously by different dolphins.

\section{$\underline{\operatorname{Scan}(E)}$}

Scanning, i.e. lateral, back-and-forth head movement, to search for or assess a target is commonly observed in captive bottlenose dolphins ( $\mathrm{Au}, 1993$ ). This behavior is difficult to observe in the field, although during underwater observations Rossbach and Herzing (1997) were able to see dolphins scanning in the clear waters of the Bahamas. In this study, the number of times a dolphin actually moved its head from side to side was not recorded, but a scanning event was scored only if an entire cycle was observed, i.e. beginning with the head in normal position followed by a continuous sweep to both sides. During a typical scan the dolphin's head moves approximately $15-20^{\circ}$ in the lateral plane.

\section{Side-swimming $(\mathrm{S})$}

In 'side-swimming' an animal rotated $90^{\circ}$ with respect to its long axis and swam normally while maintaining this rotation (Figure 4.5). Side swimming has been observed in bottlenose dolphins (Leatherwood, 1975), and other species (Caldwell, Caldwell \& Evans, 1966; Pilleri, Gihr \& Kraus, 1970). The current study elucidates an important function of this behavior, specifically its position in the foraging sequence.

\section{Accelerate (E)}

A rapid increase in speed was scored as a behavioral event and dubbed 'accelerate'. The most dramatic accelerations occurred as part of the foraging process, and it was rarely observed otherwise. Dolphins accelerated while in either normal and side-swim orientation. 


\section{Pinwheeling (E)}

Leatherwood (1975) first described this behavior in bottlenose dolphins, and he saw it most frequently in association with feeding. A 'pinwheel' is equivalent to a somersault performed by a side-swimming dolphin. To perform the turn a dolphin tucks its head and propels itself through the turn, which is a rotation around the midpoint of the body, i.e. the spine is flexed but not bent laterally (Figure 4.6). For a dolphin, the pinwheel functions as a rapid means to change direction. It was most often seen during the final stages of the foraging sequence (see Section 4.3.3), but occurred occasionally in other contexts, such as socializing.

\section{Bottom Disturbance/Bubble Cloud (E)}

This behavior is a directed effort that results in a small, local cloud of sediment being stirred up. In this behavior a dolphin swam to the bottom, caused the disturbance, and then swam back to the surface and repeated this behavior in succession. The dolphin usually produced a small bubble cloud during the same dive(s).

\section{Avoid Blimp Shadow (E)}

Dolphins under observation sometimes encountered the shadow of the aerostat, and when they made some effort to swim out of the shadow 'avoid blimp shadow' was recorded. Never did this cause an animal to be lost from observation. In most cases, an individual ceased its behavior only long enough to stay out of the passing shadow. Animals showed a similar reaction to the passing of small clouds.

\section{$\underline{\text { Look Back (E) }}$}

The 'look back' behavior is an exaggerated scan to one side, although the movement is not necessarily in the lateral plane. In this behavior a dolphin turned its head and held it in that position for 1-2 seconds. This behavior occurred during any swimming posture, but most often when a dolphin was side-swimming. Look back is interpreted as a means for more detailed assessment (visual or acoustic) of a target. 


\section{Body Contact (E)}

'Body contact' was scored any time two animals were observed to be touching. Body contact was observed readily during continuous video follows except when one animal was underneath another.

\section{Fish tossing (E)}

After capturing a prey item, dolphins were occasionally observed to 'fish toss'. With a quick jerk of its head a dolphin tossed the fish, which was usually oriented sideways in its mouth. The fish sailed approximately 1-2 $\mathrm{m}$ through the air, and the dolphin then swam to the landing spot and consumed its prey. The function of this behavior is unknown, but could involve processing of prey (Caldwell \& Caldwell, 1972). For example, the Sarasota dolphins are known to eat catfish, but typically only the catfish tails are found in stomach contents (Barros, 1992; Barros \& Wells, 1998). The dolphins therefore decapitate the fish before consuming them, and one means of accomplishing this would be a strong head jerk while holding the head, resulting in the body of the prey sailing through the air.

\section{Prey Handling (S)}

This behavioral state is simply defined as the state of a foraging dolphin after it has captured a prey item and before consuming it. This state was seldom observed as most often animals consumed prey immediately after capture, and correspondingly few behavioral events were observed during this state. One notable exception to this pattern was fish tossing, which was interpreted as part of prey handling.

\section{Formation Swimming (S)}

Two or more dolphins observed to be swimming within one body length of each other and maintaining a consistent position relative to all neighbors were said to be 'formation swimming'. No foraging behaviors (i.e. events) were observed to occur while the focal animal was in this state. Traveling animals were often observed to display this behavior. 


\section{Non-foraging (S)}

When the focal individual was obviously not engaged in any foraging behavior this behavioral state was scored. Examples of non-foraging included active socializing, virtually motionless rest at the surface, and interaction with non-food objects (boats, floating seagrass, etc.).

\subsubsection{The Sequential Nature of Dolphin Foraging}

Nineteen behaviors were considered for all $z$-score analyses: those described in the previous section as well as prey capture events and the acoustic events: echolocate, pop, and whistle (Chapter 5). These behaviors included putative foraging behaviors (e.g. 'pinwheel', 'root'), social behaviors (e.g. 'body contact'), 'neutral' behaviors (e.g. 'avoid blimp shadow'), and acoustic behaviors (e.g. 'echolocate', 'whistle'). Z-scores were calculated as described above for all behaviors in all habitats, and then for the three specific habitats in which the majority of observations were conducted: sand, sea grass meadow, and sea grass edge. The number of total pairs tallied ( $\mathrm{N}$-values), for all $\mathrm{z}$-score analyses are shown in Table 4.2 .

\begin{tabular}{|c|c|}
\hline Z-score & Total Pairs Tallied \\
\hline State lag: all habitats & 3648 \\
State lag: Habitat = Sand & 1581 \\
State lag: Habitat = Seagrass & 725 \\
State lag: Habitat = Seagrass Edge & 1063 \\
\hline
\end{tabular}

Table 4.2 Total behavioral pairs tallied for $z$-score analyses

Figure 4.7 displays the behavioral sequences of all 57 successful capture events, and Figure 4.8 shows a bifurcation diagram that splits the behavioral sequences into major types. Figure 4.9 shows the $z$-score results for all behaviors in all habitats. The $z-$ score for each behavioral pair is shown and the strength of the particular transition is indicated by the thickness of the arrow. Although not indicated in Figures 4.9-4.12, zscores less than -1.96 also resulted, indicating behavioral pair transitions that occurred significantly less frequently than expected. In the analysis for 'capture', for example, 
'scan' had a $z$-score $=-2.53$ and for 'non-foraging' the $z$-score $=-2.99$ (see Table 4.3 for matrix of negative $z$-scores). Figures $4.10-4.12$ show the state-lag results for three primary habitats. The $z$-score figures for the three habitats indicate some differences in the sequences of behaviors used and the relative importance, i.e. value of the $z$-score, of certain behavioral transitions. A chi-square analysis shows that for even a single state-lag step backwards from capture there is a significant difference between the three habitats $\left(\chi^{2}=21.33, \mathrm{df}=10, \mathrm{P}<0.02\right)$. In seagrass feeding, for example, the same three terminal behaviors occur, but 'fish whack' was much more important than in seagrass edge feeding based on its almost 4-fold larger $z$-score (Figures 4.11 and 4.12). In Figures 4.94.12 the different colored boxes for the various behaviors are meant to group behaviors relative to their minimum number of steps (i.e. transitions) away from capture. For example, accelerate is a minimum of one step from capture in Figure 4.9, but in Figure 4.12 it is at least two steps from capture.

One caveat of analyzing the sequences one step at a time is that sequences that were not observed appear as a potential pathway. For example, in figures 4.9 and 4.11 'fish whack' is preceded by 'side-swim' which often occurred and 'side-swim' is preceded by 'root' which also was common. However, the sequence 'root'-'side-swim''fish whack' did not occur. All other displayed sequences did occur. The only behaviors that preceded 'fish whack' with any significant frequency are 'side-swim', 'scan', and 'non-foraging'. Analyses of three and four step behavioral transitions are possible, but, due to the plasticity of appetitive behaviors, multi-step sequences are not likely to be deterministic which would be an assumption of multi-step analyses. In addition, the strategies utilized by the Sarasota dolphins appear to be centered around three behaviors (see Section 4.4.2), and the other appetitive behaviors are likely used as necessary, which again makes multi-step analyses problematic both in implementation and interpretation. 


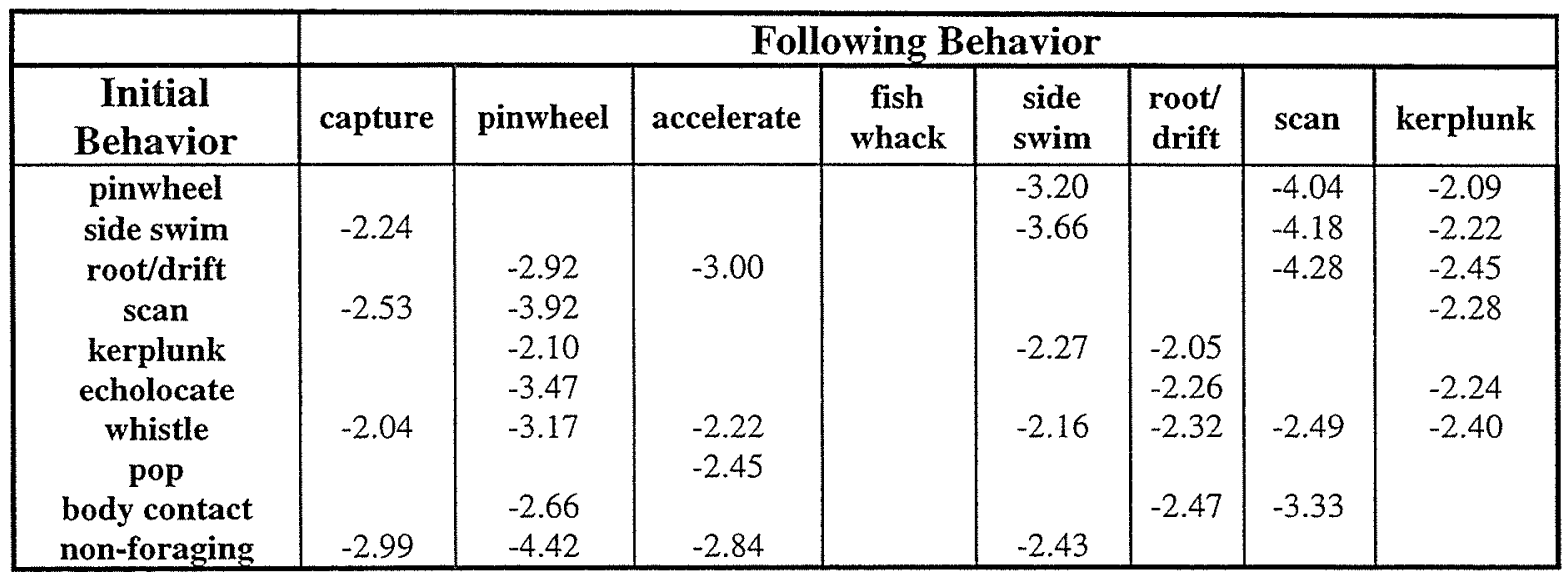

Table 4.3 Negative $z$-scores greater than -1.96. 'Target' and 'Given' are described in Section 4.2.3. Only target behaviors $<4$ steps before capture are listed because complete analyses proceeded only through four steps before each capture event. Behaviors for which no negative $z$-scores resulted have been omitted from the table.

\subsubsection{Influence of Individuals and Habitats on Observed Behavioral Patterns}

$\underline{\text { Individual preferences }}$

A contingency analysis of the behaviors used by individuals across the three primary habitats showed a highly significant difference in the use patterns $\left(\chi^{2}=904\right.$, $\mathrm{df}=136, \mathrm{P}<<0.001)$. To examine the use patterns in more detail adjusted residuals (Everitt, 1977) were calculated. The adjusted residuals were then used to estimate Pvalues for the individual cells (Table 4.4). Cells in Table 4.4 that are significantly more common than expected are shown in black, those less common than expected in red. 


\begin{tabular}{|c|c|c|c|c|c|c|c|c|c|}
\hline \multirow{2}{*}{ Individual } & \multicolumn{9}{|c|}{ Behavior } \\
\hline & Root/Drift & Side-swim & Kerplunk & Pinwheel & Capture & Accelerate & Look back & Scan & Fish whack \\
\hline FB03 & & & $<0.05$ & & & $<0.05$ & $<0.05$ & & \\
\hline FB1 1 & & & & & & $<0.05$ & & & \\
\hline FB28 & $<0.05$ & $<0.05$ & $<0.05$ & $<0.05$ & $<0.05$ & & $<0.05$ & $<0.05$ & $<0.05$ \\
\hline FB38 & & & & & & & & $<0.05$ & \\
\hline FB54 & & & & $<0.05$ & & & & $<0.05$ & \\
\hline FB65 & $<0.05$ & & & & & & & & \\
\hline FB75 & & & & & & & $<0.05$ & & \\
\hline FB79 & & & & & & & & $<0.05$ & \\
\hline FB83 & & & & & & & & $<0.05$ & \\
\hline FB92 & $<0.05$ & & $<0.05$ & $<0.05$ & & & & & \\
\hline F101 & & & & & & & & $<0.05$ & \\
\hline F118 & & & $<0.05$ & $<0.05$ & & & $<0.05$ & & $<0.05$ \\
\hline F138 & & & & & & & & & \\
\hline F155 & & $<0.05$ & $<0.05$ & $<0.05$ & & $<0.05$ & & $<0.05$ & $<0.05$ \\
\hline F157 & & & $<0.01$ & $<0.05$ & & & & $<0.05$ & \\
\hline UNK2 & & & & & & & & & \\
\hline UNK3 & $<0.05$ & & $<0.01$ & $<0.05$ & & & & $<0.05$ & \\
\hline
\end{tabular}

Table 4.4 P-values (from adjusted residuals) for the use of behaviors by individuals. Cells significantly more common than expected are shown in black, those less common in red.

\section{Habitat influence}

A chi-square analysis showed highly significant differences in the relative occurrences of these behaviors between habitats $\left(\chi^{2}=247, \mathrm{df}=18, \mathrm{P}<0.001\right)$. In a more detailed analysis using multiple condensed contingency tables, the occurrences of each foraging behavior in each of the three most common habitats were analyzed independently against the occurrences of the rest of the foraging behaviors in that and the other two habitats. For example, the occurrence of root/drift in sand was compared to the occurrence of root/drift in seagrass and seagrass edge and the occurrence of the rest of the foraging behaviors with a similar habitat breakdown (Table 4.5). The resulting P-values are summarized in Table 4.6. It is noteworthy that pinwheel is the only behavior favored in more than one habitat, all others are favored in only one and often disfavored in others. 


\begin{tabular}{|c|c|c|}
\hline Habitat & Root/Drift & $\begin{array}{c}\text { All other foraging } \\
\text { behaviors }\end{array}$ \\
\hline $\begin{array}{c}\text { Sand } \\
\text { Seagrass + Seagrass Edge }\end{array}$ & 139 & 350 \\
\hline
\end{tabular}

Table 4.5 Example of individual $2 \times 2$ contingency table analyses for the habitat-specific occurrence of foraging behaviors.

\begin{tabular}{|c|c|c|c|c|c|c|c|c|c|}
\hline \multirow[b]{2}{*}{ Habitat } & \multicolumn{9}{|c|}{ Behavior } \\
\hline & root/drift & $\begin{array}{l}\text { side- } \\
\text { swim }\end{array}$ & kerplunk & pinwheel & capture & accelerate & scan & $\begin{array}{c}\text { bottom } \\
\text { dist/bubble }\end{array}$ & $\begin{array}{c}\text { fish } \\
\text { whack }\end{array}$ \\
\hline Sand & $\leq 0.001$ & & $\leq 0.001$ & $<0.001$ & & & $\leq 0.001$ & $<0.01$ & $\leq 0.001$ \\
\hline Grass & & $\leq 0.001$ & $\leq 0.001$ & & & & & & $<0.001$ \\
\hline Edge & $<0.001$ & $<0.01$ & $\leq 0.001$ & $<0.05$ & & & & $\leq 0.001$ & \\
\hline
\end{tabular}

Table 4.6 $\chi^{2} P$-values comparing each foraging behavior with all others across habitats. Cells for which observed counts were significantly greater than expected are in black, those less common than expected in red. P-values less $<0.01$ are shown in bold, and those $<0.001$ are also underlined.

\subsubsection{Nearest Neighbor Distance and Occurrence of Foraging Behaviors}

The distance from the focal animal to its nearest neighbor was scored continuously during review of videotapes. Nearest neighbor distance (NND) was a separate category in the Observer ${ }^{\circledR}$ protocol, and a focal dolphin was scored to be in one of six mutually exclusive distance categories: $<1 \mathrm{~m} ; \leq 2 \mathrm{~m} ; \leq 5 \mathrm{~m} ; \leq 10 \mathrm{~m}$; $>10 \mathrm{~m}$; or 'single animal'. Table 4.7 shows the occurrence of foraging behaviors as a function of NND. For these analyses focal females with dependent calves were removed from the data because the proximity of the calf (as nearest neighbor) is likely to be a special case.

Comparing all foraging behaviors together with non-foraging relative to NND revealed a dependent relationship $\left(\chi^{2}=14.87, \mathrm{df}=4, \mathrm{P}<0.01\right)$. A comparison of only foraging, multiple-animal groups ('non-foraging' behavior and 'single animal' categories removed) showed in a significantly dependent relationship between foraging behaviors and NND $\left(\chi^{2}=96.98, \mathrm{df}=36, \mathrm{P}<<0.001\right)$. More detailed analyses revealed relationships between specific behaviors and NND. 


\begin{tabular}{|c|c|c|c|c|c|c|}
\hline & \multicolumn{7}{|c|}{ Distance of focal's nearest neighbor } \\
\cline { 1 - 5 } & $<\mathbf{1}$ & $\leq \mathbf{2}$ & $\leq \mathbf{5}$ & $\leq \mathbf{1 0}$ & $>\mathbf{1 0}$ & Single \\
\hline Behavior & 110 & 120 & 64 & 31 & 17 & 95 \\
Non-foraging & 12 & 31 & 31 & 8 & 8 & 111 \\
Root/drift & 41 & 42 & 29 & 6 & 5 & 97 \\
Side-swim & $13(9)$ & $26(19)$ & $9(6)$ & 0 & 0 & 5 \\
Kerplunk & 1 & 24 & 28 & 3 & 3 & 101 \\
Pinwheel & 8 & 13 & 8 & 1 & 1 & 28 \\
Capture & 12 & 11 & 16 & 3 & 1 & 66 \\
Accelerate & 6 & 4 & 1 & 1 & 0 & 8 \\
Look back & 47 & 40 & 29 & 14 & 6 & 116 \\
Scan & $16(10)$ & $12(7)$ & 0 & 0 & 0 & 0 \\
Fish whack & $16(10)$ &
\end{tabular}

Table 4.7 Distance (meters) of focal animal's nearest neighbor and occurrence of foraging behaviors. Data for females with dependent calves have been removed. Behaviors above the line in column one are states, below are events. Values in parentheses indicate the subset of kerplunk and fish whack that occurred synchronously with another dolphin.

\begin{tabular}{|c||c|c|c|c|c|}
\hline Behavior & $\begin{array}{c}\text { All nearest } \\
\text { neighbor } \\
\text { distances }\end{array}$ & $\begin{array}{c}<1 \mathrm{~m}- \\
\leq 2 \mathrm{~m}\end{array}$ & $\begin{array}{c}\leq 2 \mathrm{~m}- \\
\leq 5 \mathrm{~m}\end{array}$ & $\begin{array}{c}\leq 5 \mathrm{~m}- \\
\leq 10 \mathrm{~m}\end{array}$ & $\begin{array}{c}\leq 10 \mathrm{~m}- \\
>10 \mathrm{~m}\end{array}$ \\
\hline root/drift & $<\mathbf{0 . 0 1}$ & $<\mathbf{0 . 0 5}$ & 0.198 & 0.822 & 0.34 \\
\hline side-swim & 0.531 & 0.212 & 0.73 & 0.726 & 0.683 \\
\hline kerplunk & $<\mathbf{0 . 0 5}$ & 0.177 & $<\mathbf{0 . 0 5}$ & 0.133 & na \\
\hline pinwheel & $\mathbf{0 . 0 0 1}$ & $<\mathbf{0 . 0 0 1}$ & $<\mathbf{0 . 0 5}$ & 0.139 & 0.598 \\
\hline capture & 0.914 & 0.609 & 0.663 & 0.525 & 0.769 \\
\hline accelerate & 0.439 & 0.383 & 0.0695 & 0.686 & 0.526 \\
\hline look back & 0.351 & 0.284 & 0.302 & 0.268 & 0.41 \\
\hline scan & $<\mathbf{0 . 0 5}$ & $<\mathbf{0 . 0 5}$ & 0.906 & $<\mathbf{0 . 0 5}$ & 0.2636 \\
\hline fish whack & $\mathbf{< . 0 0 1}$ & 0.128 & $<\mathbf{0 . 0 1}$ & na & na \\
\hline
\end{tabular}

Table $4.8 \chi^{2}$ P-values for $2 \times 2$ contingency table analyses of foraging behavior and NND. See text for details of interpretation.

Table 4.8 tabulates the results of condensed contingency table analyses comparing each behavior with all others combined against progressive pairs of NNDs. For example, 'kerplunk' compared with all other foraging behaviors at NNDs of $\leq 2 \mathrm{~m}$ and $\leq 5 \mathrm{~m}$ resulted in a $\mathrm{P}<0.05$. NND was categorized into the above groups (Table 4.7), and the 
condensed contingency table analyses (Table 4.8) investigate 'break points', i.e. the distance category or categories at which there is a significant change in the occurrence of a particular behavior. The break point occurs at the $<1-\leq 2 \mathrm{~m}$ point for root/drift and scan, and at the $\leq 2-\leq 5 \mathrm{~m}$ point for kerplunk and fish whack. Root/drift was more common than expected after its break point, i.e. it occurred more often than expected as animals spread out. Scan, fish whack, and kerplunk were less common than expected after their individual break points (Table 4.8), i.e. they occurred more than expected when animals were relatively close together. Pinwheel had a unique distribution; it was less common than expected at $<1 \mathrm{~m}$, more common than expected at $\leq 2 \mathrm{~m}$ and $\leq 5 \mathrm{~m}$, and then less common thereafter. The distributions of the other behaviors (side-swim, capture, accelerate, and look back) did not differ significantly with NND.

\subsubsection{Foraging behaviors occurring during other activities}

Traditionally, Sarasota dolphin behavior is categorized into one of five activity states or events: travel, social, feed, probable feed, rest and mill (Urian \& Wells, 1996). The methods of the present study permitted the collection of foraging behaviors separately but simultaneously with general activity. Figure 4.13 shows the rate of occurrence of foraging behaviors during the different general activities. Relatively high rates of some foraging behaviors occurred during activities that were not strictly defined as foraging activities. These activities, however, were suspected to have some foraging component due to the obvious feeding behaviors that often punctuate them. Most notable are the rates of scan and root during traveling, the rates of scan, accelerate, pinwheel, side-swim, and root during milling, and, while not as surprising, are the high rates of most foraging behaviors during probable feed. This last activity state is a conservative definition of feeding. Given the sub-surface occurrence of many of the foraging behaviors and the previous lack of a direct link to feeding, it is not surprising that many foraging behaviors occur during this suspected feeding state. 


\subsection{Discussion}

\subsubsection{Foraging Behavior Sequences}

In the overall analysis beginning with a capture event and retreating backwards in time, a variety of pathways lead from the search stage, through pursuit, and finally to a capture. The goal of this portion of the study was to begin to build a foraging ethogram, and the 'backwards' analysis has accomplished this by defining which behaviors lead to prey capture. With significant links demonstrated between prey capture events and the behaviors indicated in Figures 4.9-4.12, analyses of the ecology of these and related behaviors (e.g. sound use) as foraging activities can be conducted with confidence. In addition, other behaviors, either newly or previously observed, can be more confidently associated with feeding if seen to occur in significant relationship with other linked behaviors.

While this 'backwards' analysis definitively links specific behaviors with the foraging sequence, it does not account for decision points or failed sequences (but see Figure 4.7 for repeated, i.e. potentially failed, sequences). Therefore, with this analysis, data necessary for optimal foraging models (e.g. encounter rates) are not attainable. Having defined the stages of foraging, however, this study makes possible the forward analysis, which can be used to obtain quantities for optimal foraging models. Behaviors occurring immediately before a capture are interpreted as pursuit behaviors. In the overall analysis we see three obvious pursuit behaviors: 'pinwheel', 'fish whack', and 'accelerate'. The large $z$-scores for the first two behaviors demonstrate their importance in the final stages before capture. These behaviors may be used in response to evasive behaviors performed by the prey. Reflexive evasive behaviors causing an unpredictable heading change are common among fish (Blaxter, Gray \& Denton, 1981; Eaton, Bombardieri \& Meyer, 1977; Zottoli, 1977). Evasive maneuvers by prey may result in few straight, fast chase sequences (Figure 4.8). In shallow water or near-surface feeding, the pinwheel and fish whack behaviors both could account for the commonly seen flurry 
of white water and 'fast, non-directional' behaviors observed by other researchers (Felleman, Heimlich-Boran \& Osborne, 1991).

The next step backward from pursuit and capture in the foraging process is 'search'. The $z$-score analyses, however, do not precisely distinguish which behaviors should be considered 'search' and which are 'pursuit'. Side-swim, for example, is of central importance to all pathways and is interpreted as a pursuit behavior given its close association with all three terminal behaviors and the fact that it often follows accelerate. Kerplunk and scan, however, occur earlier in the sequence and relatively more often than side-swim without being followed by one of the obvious pursuit behaviors. Additionally, scan is used to search for and/or assess targets ( $\mathrm{Au}, 1993)$, and, while its function is less well understood, kerplunk is interpreted as a flushing behavior. A kerplunk likely contains the frequencies and energy necessary to evoke a startle or 'C-start' response in fish (Blaxter et al., 1981; Blaxter \& Hoss, 1981; Eaton et al., 1977). This reaction causes a fish to move rapidly, making detection by either echolocation or vision more likely than if the fish is motionless in the cluttered environment of a seagrass bed. Further evidence for kerplunks being used to flush fish is their extensive use at the edges of seagrass beds, which are used as a refuge by fish (Sogard \& Olla, 1993). For these reasons scan and kerplunk are considered to be part of the 'search' stage with the other behaviors such as root, drift, and look back which are more obviously part of the initial stage of foraging. Many of these results suggest that dolphins use some active mechanism to flush prey, but other predation strategies also occur as indicated in the $z$-score analyses. Look back is significantly linked to side-swim in the overall assessment (Figure 4.9) and in seagrass feeding (Figure 4.11). This behavior has been interpreted as a dolphin more closely inspecting a target, so perhaps this represents a more opportunistic search strategy with a dolphin tracking an already moving or detected target. Beyond look back other foraging strategies (e.g. sit-and-wait) were rarely observed, although anecdotal observations suggests that Sarasota dolphins do utilize other strategies. On one occasion a single (i.e. no others within $1 \mathrm{~km}$ ) dolphin was observed catching a fish in the mode of a sit-and-wait 
predator. The individual was in shallow water $(<1 \mathrm{~m})$ and very near shore $(<1 \mathrm{~m})$ in a sparse seagrass bed. It remained motionless for approximately 10 seconds and then lunged forward almost beaching itself as it caught a fish. Dolphins in other areas have been observed to chase fish up onto an embankment and beach themselves to catch their

prey (Hoese, 1971; Petricig, 1995). It appears that this Sarasota dolphin similarly used the shoreline to corner its prey, although it did not chase the fish onto the beach but waited for the fish to come between it and the shoreline. 'Sea wall foraging' is another foraging strategy seen infrequently during the current study, but it has been observed over many years (R. Wells, pers comm). During sea wall foraging animals swim very fast back-and-forth parallel to the sea wall, then turn and swim directly at the wall veering off just before contacting the wall, and then often catch a fish. As evidenced by these examples, other foraging strategies exist within the Sarasota dolphin population. The current study has simply investigated in depth some subset of the foraging strategies utilized by Sarasota dolphins.

\subsubsection{Distinguishing Characteristics of Foraging Behaviors: Individual and Habitat Influences and Indications for Distinct Foraging Strategies}

The occurrence of the foraging behaviors observed during the current study is affected by at least three factors: habitat, individual, and NND. The dolphins inhabiting Sarasota Bay utilize at least three distinct foraging strategies: root/drift, kerplunk, and fish whack. Two of the three techniques (root/drift and kerplunk) share a pursuit and capture sequence but differ in their method of searching for or flushing prey. The third variation (fish whack) shows little similarity to the other two throughout the foraging sequence, although it shares a pattern with kerplunk as animals tend to display it when their nearest neighbor is $<5 \mathrm{~m}$ away (see Section 4.4 .3 for further discussion). Evidence for three distinct strategies is multi-faceted. Capture events are not habitat-specific, but each of the three search strategies occurs significantly more than expected in a single habitat, and each is specific to a different habitat (Table 4.6). Also distinguishing the three strategies are NND (Table 4.8) and individually-specific preferences (Table 4.4). 
The search behaviors appear to flush prey from their refuges within a grass bed or on the bottom. Kerplunking, for example, likely produces physical forces and sound, either or both of which might cause a startle response causing a prey item to move enough to then be detected acoustically or visually. Rooting directly flushes prey from their refuges and may even lead to capture within the sediment (Rossbach \& Herzing, 1997). Rooting occurred significantly more than expected in sand, less than expected at the seagrass edge, and not significantly different than expected in seagrass. While fish utilize sea grass beds for refuge (Sogard \& Olla, 1993), adult fish are known to venture beyond the bounds of the seagrass meadows (Savino \& Stein, 1989) and most fish prey of bottlenose dolphins are bottom dwellers (Barros \& Odell, 1990; Wells \& Scott, 1999). Another possible target prey for a rooting dolphin could be burrowing benthic invertebrates. Dolphins rooting near crab traps may also be targeting invertebrates growing on the traps or attracted to their contents. A fish whacking dolphin, on the other hand, does not directly flush prey but appears to take advantage of fish schools in the water column above seagrass meadows. Fish whacking appears to be more opportunistic or tied to schooling prey that are more detectable in as much as the dolphins do not appear to use a specific behavior to flush prey, i.e. it is preceded only by side-swim, scan, and non-foraging activity. They do, however, choose the seagrass habitat very specifically for fish whacking (Table 4.6), which shows purposive intent indicative of appetitive behaviors (Tinbergen, 1951).

A striking feature of Sarasota dolphin foraging is the presence of distinctive patterns of foraging behaviors displayed by different individuals within the population. Table 4.4 shows that some animals favor or disfavor certain behaviors. With regards to the three suggested distinct foraging strategies: root/drift, kerplunk, and fish whack, no animal shows significant preference for more than one of these behaviors. In addition, animals that do prefer one of them disfavor one or both of the other two. Some behaviors are used by all animals indicating that they may be of general importance to all foraging 
strategies (e.g. side-swim, accelerate). The analyses presented indicate that the pattern of occurrence of a particular behavior is dependent on both the individual performing the behavior and the habitat in which it occurs. The use of a particular foraging strategy in oystercatchers is similarly affected (Goss-Custard \& Le V dit Durell, 1983), but is also initially dictated by a bird's parents (Norton-Griffiths, 1967). Two cases in this study may point toward parental influence on foraging behavior. Focal animal F155 showed preference for fish whacking (Table 4.4). Her mother (FB05) was commonly seen fish whacking during this study but was not included in the individual analyses as her "video minutes' total was less than the 30 minute minimum. She has often been observed fish whacking with another of her daughters, FB55. The mother of the other 'fish whacker', FB28, is unknown. In the other instance, one of the 'kerplunkers', F157, had a dependent calf during 1996. In 1997 this calf (F186), while swimming approximately $2 \mathrm{~m}$ behind F157, kerplunked four times each within 5 seconds of F157 performing the same behavior. F157 has been observed kerplunking in Tampa Bay as early as 1990, and she has recently begun to use Palma Sola Bay (part of the Sarasota study area) during the summer (R. Wells, pers. comm.).

Kerplunking also may be associated with some regional specificity. The two animals in this study that strongly favor the behavior are two of the three focal animals most commonly sighted in the adjacent waters of Tampa Bay. In addition, many of the core 'Sarasota' animals use this behavior significantly less frequently than expected when compared to the rest of the behaviors (Table 4.4). While there is no physical boundary between the 'Sarasota' and 'Tampa Bay' dolphin communities, kerplunking may be a behavior that is more successful in Tampa Bay due to some difference in habitat or prey distribution. Such foraging specializations in apparent response to differing prey fields has been observed in other cetaceans (Weinrich, Schilling \& Belt, 1992). The Tampa and Sarasota animals may, on the other hand, constitute different populations that have experienced different local environmental conditions long enough to have locally specific behaviors. Weinrich et al. (1992) suggest that the new foraging strategy they observed 
passed quickly through a local population via some learning mechanism, and Galef (1995) describes how such socially learned behaviors are adaptive to local conditions.

The current study provides anecdotal evidence that foraging strategies are passed from mother to offspring, although the young animals could acquire the strategy from other individuals in the population. It is difficult to resolve the means by which an individual 'learns' a foraging strategy because many mechanisms are available for the passage of acquired behaviors (Whiten \& Ham, 1992). To investigate the mechanisms of the acquisition of 'local' behaviors in dolphins would require longitudinal data documenting the behavioral repertoires of animals with whom a young individual associates and the association patterns it has with those animals.

Individuals in the Sarasota population do possess different as well as overlapping repertoires of foraging behaviors. Fish whacking and kerplunking, for example, were observed in only few focal animals in this study (fish whack: 2 of 17; kerplunk: 3 of 17), but rooting was observed in 16 of 17 . Small sample sizes precluded a multi-dimensional analysis of variance, which could have been used to address the question of whether the driving force behind observed behaviors was that individual's repertoire, the habitat, or some other factor(s). For example, if individuals are locked into limited repertoires of foraging behaviors, do individuals target habitats most appropriate for their repertoire? Alternatively, perhaps the animals have larger repertoires than those observed in the current study. If so, does an individual encountering a certain habitat or prey refuge type simply select the foraging strategy most appropriate for that environment? The data presented here represent only a short snippet of time in the lives of these animals, so the focal animals may display a larger repertoire than observed, or perhaps further observation would reveal that they do indeed have limited repertoires and target specific habitat or prey refuge types. 


\subsubsection{Nearest Neighbor Distance and Foraging Behavior}

Previous reports of bottlenose dolphins have stated that the animals 'spread out when foraging' (Irvine et al., 1981), but no quantitative description has been offered. This lack of quantitative data likely stems in part from not knowing definitively when the animals are foraging. The results of the current study point to very specific instances in which animals change their inter-animal distance while foraging. Whether and how much an animal distances itself from its nearest neighbor appears to be associated with foraging strategy. Five behaviors (root/drift, kerplunk, pinwheel, scan, and fish whack) are all significantly affected by NND overall, but they are affected differently depending on absolute distance (Tables $4.7 \& 4.8$ ). The occurrence of root/drift is significantly different only when the spread changes from $<1 \mathrm{~m}$ to $\leq 2 \mathrm{~m}$ being much less common than expected at $<1 \mathrm{~m}$ and more common than expected as animals spread out. This strategy appears to function as a physical means of probing the sediment to flush prey as has been observed in other areas (Rossbach \& Herzing, 1997). The Sarasota dolphins were not observed to bury their rostra or heads as Rossbach and Herzing (1997) observed, but the behavior certainly appears to be focused on flushing single prey from a benthic refuge. Given this emphasis, and the fact that root/drift occurred 1.5 times more often in single animals than in all multi-animal observations (Table 4.7), it is not surprising that an animal would distance itself from others while engaged in this foraging strategy.

Kerplunk and fish whack share a pattern of dependence on NND that is different from root/drift (Tables $4.7 \& 4.8$ ). The first two strategies occur only when an animal is relatively close to conspecifics, i.e. the animals spread out more to root/drift than to kerplunk or fish whack. All three depend significantly on NND overall, but the break point occurs earlier (i.e. smaller NND) for root/drift than for kerplunk and fish whack. Neither kerplunk nor fish whack occurs at all when animals are $>5 \mathrm{~m}$ apart and the distributions between $<1-\leq 2 \mathrm{~m}$ are not significantly different than expected. In other words, the occurrence of these two behaviors is affected by NND only inasmuch as they occur far less than expected (kerplunk) or not at all (fish whack) when animals are more 
than $5 \mathrm{~m}$ apart. These results, and the fact that $71 \%$ of kerplunks and $61 \%$ of fish whacks occurred synchronously with at least one other animal, suggest a cooperative or social component to these two foraging strategies as opposed to root/drift which appears to be more solitary.

Kerplunks likely produce a loud, low frequency sound and certainly entrain a mass of bubbles. The loud low sound could be sufficient to elicit a startle response in fish as discussed above, and/or the bubbles could act as a boundary against which the dolphin could trap their prey as has been observed in humpback whales (Hayes, Winn \& Petricig, 1985; Jurasz \& Jurasz, 1979; Sharpe \& Dill, 1997). This behavior occurs most frequently in seagrass edges and is likely directed at prey fish hiding in the seagrass, which is relatively acoustically and visually opaque to dolphins. The large number of kerplunks that occur synchronously suggests that this strategy is used to flush prey from the seagrass refuge, and that this strategy is most effective with additional animals at least patrolling or possibly also kerplunking. The spacing of the animals could be important when considering the bubble effects. If the bubbles are an important component, then spacing between animals that is too great could decrease the effectiveness of the strategy. In addition, one kerplunk may flush many fish in which case it may be beneficial for a nearby animal whether or not it is kerplunking.

Fish whacking was not observed to occur when NND>2 m, and $61 \%$ occurred simultaneously with at least one other fish whack. These data suggest some cooperative aspect of this foraging strategy. Fish whack, however, is significantly more common in seagrass than in the other two habitats which differs starkly from kerplunk (Table 4.6). Also, this behavior always involved predation on a school of prey rather than single prey as in root/drift. The need for multiple, closely spaced animals may be two-fold for fish whack. This strategy appears to rely upon a school of fish (minimum size unknown) being flushed from within the seagrass as animals swim over the meadow, and this flushing could possibly be more effective with multiple animals creating a surface 
pressure wave across the seagrass. After flushing fish, a dolphin will fish whack in the middle of the fish school sometimes propelling fish out of the water. Even if a single fish is not hit hard enough to propel it from the water, the products of the event (e.g. sound, pressure, and/or bubbles) appear to disorient the fish sufficiently so as to become easier prey. As discussed above the only other foraging behaviors significantly linked to fish whack are scan and side-swim.

\subsubsection{New insights into Sarasota dolphin foraging}

Irvine (1981), Waples (1995), Wells (1991), and Barros and Wells (1998) have described several aspects of foraging behavior in Sarasota dolphins. For example, these studies found that animals spread out to feed in seagrass meadows (Irvine et al., 1981), and that these seagrass beds are particularly important to foraging Sarasota dolphins, especially in the summer (Barros \& Wells, 1998; Waples et al., 1995). These studies formed the basis for the general model that Sarasota dolphins are solitary hunters that feed on individual prey items. The current work supports both of these conclusions, but presents evidence of a slightly more complex ecology. This study has revealed foraging strategies that are focused on exploiting predictable prey refuges, which leads to a different overall model for finding prey.

Specifically, this work has found that while seagrass meadows are important for Sarasota dolphins foraging using certain strategies, other strategies utilize different habitats (e.g. kerplunking at seagrass edges). Likewise, some foraging strategies did occur more frequently as dolphins spread out and became 'solitary' (e.g. rooting), but others were more common when animals were close together and often occurred synchronously (e.g. fish whacking). Finally, the assertion that Sarasota dolphins hunt for individual prey is also supported in some cases, but again certain strategies appear to target schooling prey (e.g. fish whacking). Two revisions to the current understanding of Sarasota dolphin foraging are evident. First, the model that describes Sarasota dolphins as solitary hunters targeting individual prey in seagrass meadows should be expanded include the occurrence of one or more dolphins searching a prey refuge and working to 
flush the prey from or corral them within the refuge. Kerplunking, for example, targets the edge of the refuge where fish that wander out of the seagrass meadow but re-enter as the dolphin approaches would be found. To find a single fish is a more difficult problem than to simply go to a refuge where prey density is often high. Such refuges could be located in a variety of ways including vision, echolocation, or perhaps memory.

The description of coordinated social feeding is a significant new finding for Sarasota dolphins and represents the second revision in the understanding of Sarasota dolphin foraging behavior. No previous reports have documented the occurrence of coordinated foraging strategies in Sarasota dolphins, but fish whacking and perhaps kerplunking represent just such strategies. Other studies have reported coordinated feeding strategies in dolphins from other areas (Hoese, 1971; Petricig, 1995; Würsig \& Würsig, 1980). These specialized strategies are so highly synchronized that participants presumably need time not only to specialize but also to learn cues that facilitate synchronization. Connor (1992) described the synchronous behavior of male dolphins in Monkey Mia. In this case the goal of their behavior was to maintain control over and therefore access to females. Are similarly synchronous behaviors used in different contexts by the same individuals, i.e. do participants display similar synchronous behaviors for mate guarding as well as foraging? Pairs of male dolphins do form consortships with females in Sarasota (Moors, 1997), and a relatively new male pair was observed to fish whack synchronously. What are the costs and benefits of acquiring and synchronizing these behaviors? Perhaps less time is required for foraging, more prey caught per unit effort, and/or perhaps other social benefits result from coordinating with a conspecific?

\subsubsection{Comparison of boat-based vs. overhead observations: an aide for the boat-based observer}

If an observer can see an animal for only a small portion of the total observation time and most of those glimpses occur when the dolphin comes to the surface to breathe, it would be surprising if that behavioral sample was representative. This is the case for the behavioral observer in Sarasota, where the water opacity often precludes seeing a 
dolphin below the water's surface. The current study confirmed that, in fact, boat-based observations do miss many behaviors. Many of the behaviors reported in this study that occur below the surface have not previously been observed in Sarasota dolphins (e.g. root/drift, scan). The overhead video has also provided a deeper understanding of other behaviors described for the Sarasota population as well as others. Fish whacking, for example, was known to be an important prey capture technique (Wells et al., 1987), but an understanding of its behavioral precursors, the importance of NND and habitat, and the sequence of maneuvers of the behavior has been facilitated by the overhead perspective. Root has also previously been described for bottlenose dolphins (Shane, 1990), but its direct link to feeding and the habitat and frequency of its occurrence were not well understood with only boat-based observations.

How can this new information benefit the boat-based observer? Elucidating the sequences of foraging behaviors and demonstrating that each is significantly linked to feeding may be the most important contribution. With this information an observer who sees one of the feeding-linked behaviors can confidently record that an animal is foraging. Shane (1990) used a travel/feed behavioral state to describe what she believed to be a combination of the two states. This study supports that link as scan and root occurred relatively frequently during travel as well as other general activities (Figure 4.13). Perhaps a traveling dolphin scans to assess its location and hence to navigate, and a product of the information gathered (by echolocation or vision) is detection of prey. This result could also be explained by a dolphin traveling in an 'opportunistic foraging' mode, whereby it may choose to pursue prey detected while traveling. Traveling may, however, exclude foraging behaviors as in the case of formation swimming.

Behavioral observations are limited to behavior(s) that are readily seen and easily confirmed. Cetacean researchers, in most cases, have been limited to observing surface and near-surface behaviors, and have defined the data they collect according to what is available to them. The Sarasota Dolphin Research Project (SDRP) has developed and 
used such definitions (Bassos, 1993; Moors, 1997; Urian \& Wells, 1996; Waples et al., 1995; Wells, Irvine \& Scott, 1980; Wells et al., 1987). Behavioral states have been defined using cues such as locomotion patterns (e.g. 'mill': non-directional movement that often occurs in conjunction with other activities). These categories are necessarily conservative, relatively easy to observe and score, but, because surface and underwater behavior are not always linked, the activity classifications are not always indicative of function.

The relatively low rate of occurrence of most foraging behaviors during 'feed' is a result of the fact that 'feed' is defined by SDRP as the event of a dolphin observed with a fish in its mouth (Urian \& Wells, 1996). For this study feed was a state initiated when a dolphin caught a fish at the surface and continued for $30 \mathrm{sec}$. at which time it was discontinued unless another feeding event occurred. This explains the relatively frequent occurrence of side-swim and pinwheel.

'Probable feed' is a state defined by SDRP as 'indications of feeding without confirmation (i.e. fish in the dolphin's mouth) that feeding has occurred.' With data showing the frequent occurrence of virtually every foraging behavior during probable feed (Figure 4.13), many of which would not be visible by a boat-based observer (e.g. root, scan), this study supports an expansion of the current activity category. Given the high rates of occurrence of many foraging behaviors, if an observer sees any of the behaviors significantly linked to a prey capture then perhaps the behavioral event 'feed' should be scored as the behavioral state. Likewise during 'mill' many foraging behaviors not necessarily visible from the surface were observed to occur (Figure 4.13). Often dolphins did simply mill, displaying none of the behaviors defined here as foraging behaviors, and in that case the current conservative definition is appropriate. If, however, a boat-based observer can confirm the occurrence of one of the behaviors significantly linked to capture, then perhaps the expanded 'feed' state is more appropriate. 


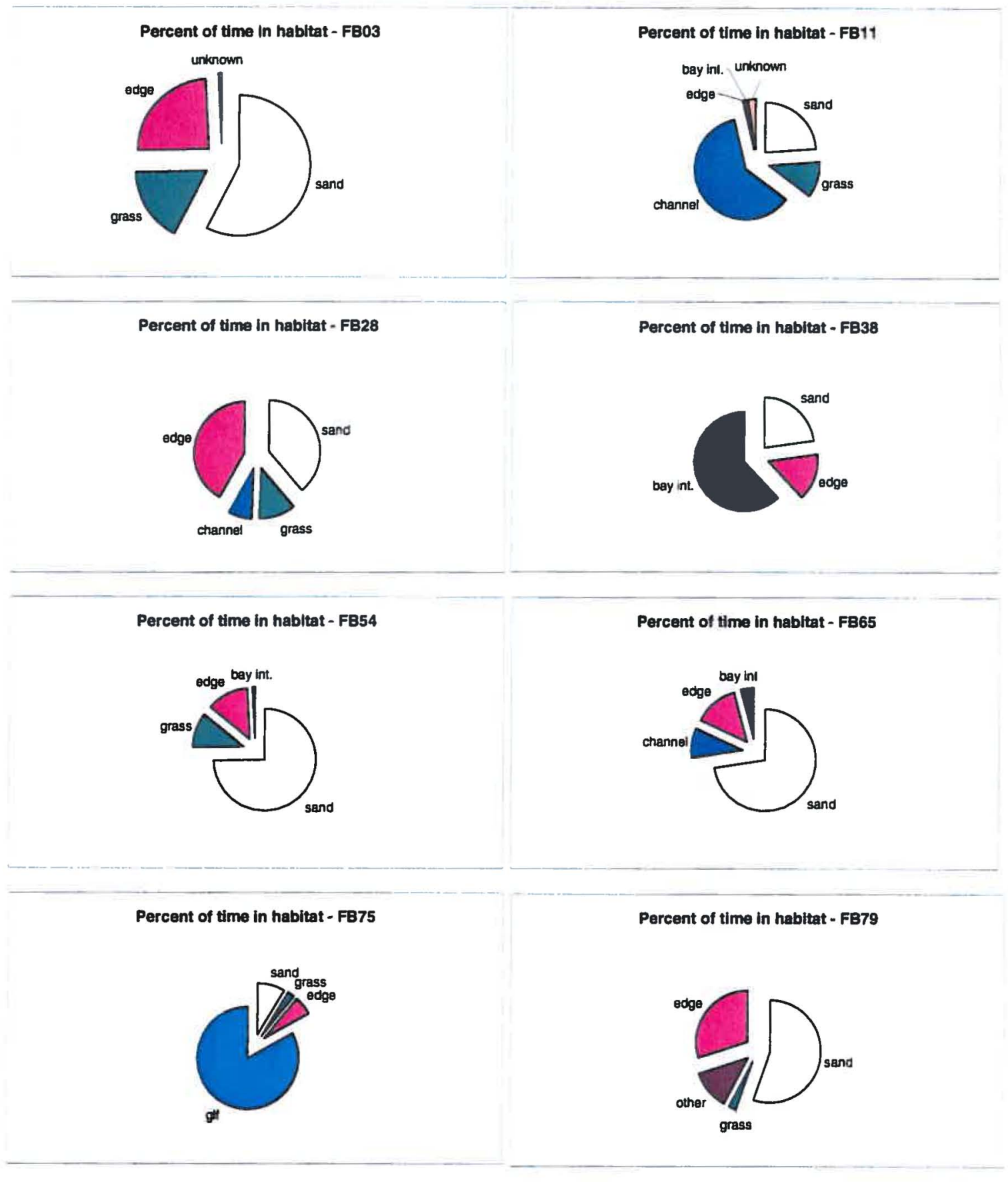

Figure 4.1a Habitat use by focal animals 
Percent of time in habltat - FB83

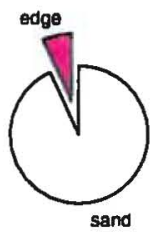

Percent of time in habltat - FB92

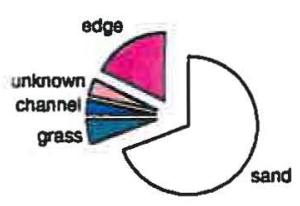

Percent of time in habitat - FB101

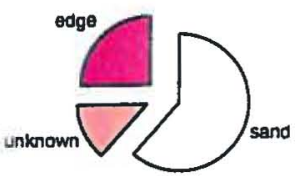

Percent of time in habltat - FB138

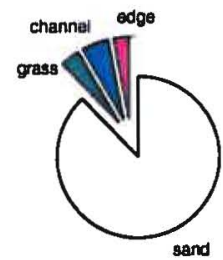

Percent of time in habitat - FB157

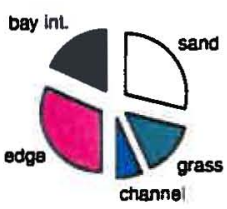

Percent of time in habltat - FB118

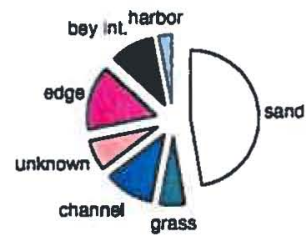

Percent of time in habitat - FB155

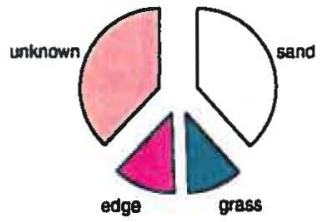

Percent of time in habital - Unknown ":2

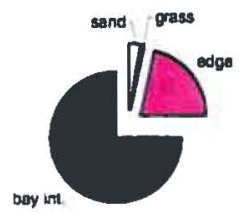

Percent of time in habltat - Unknown \#3

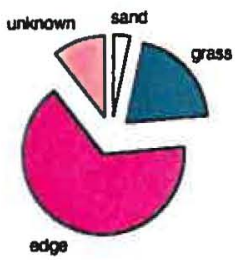

Figure 4.1 b Habitat use by focal animals 


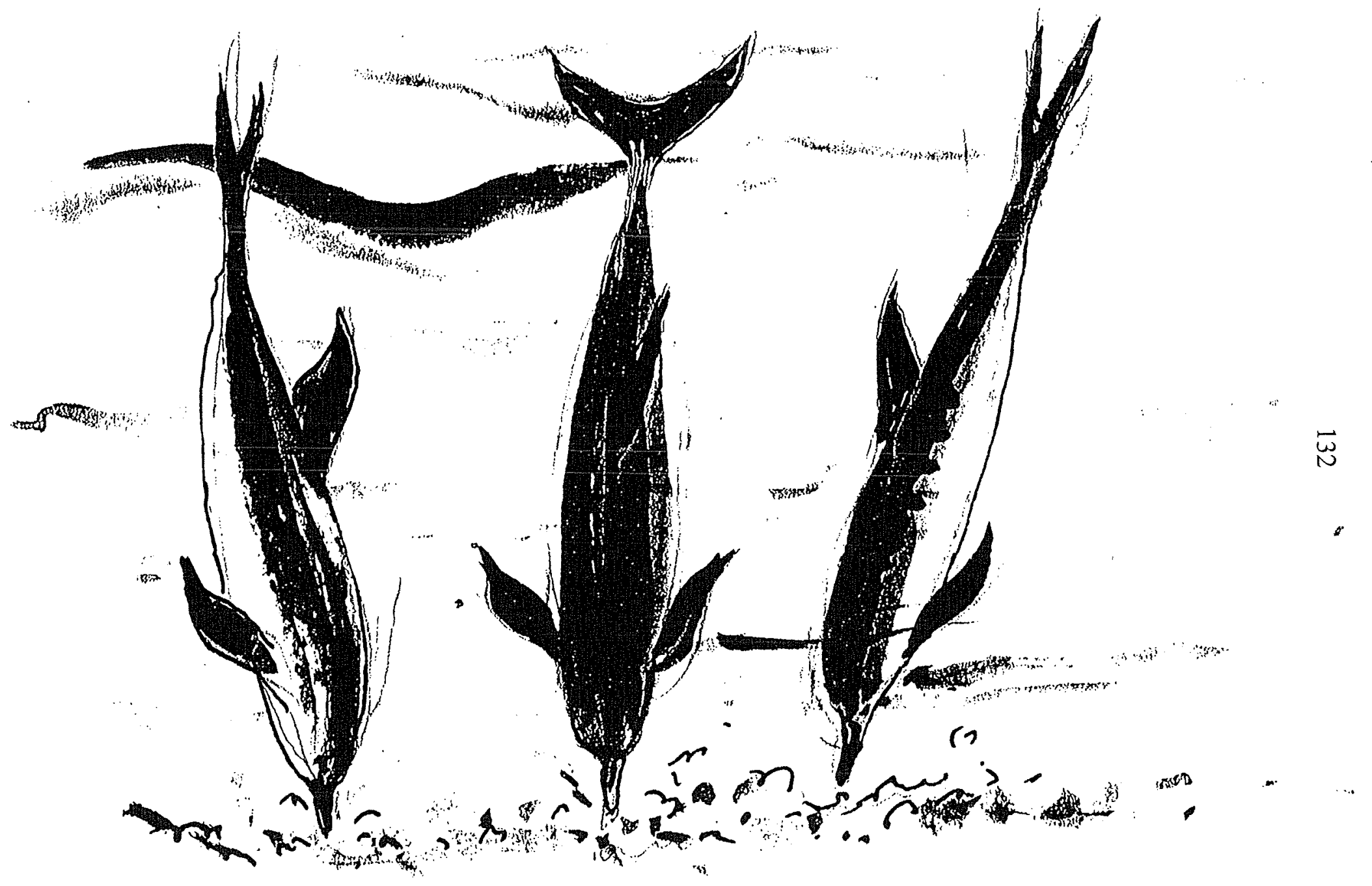

Figure 4.2 'Root' from the perspective of an underwater viewer observing the animals at the bottom. 


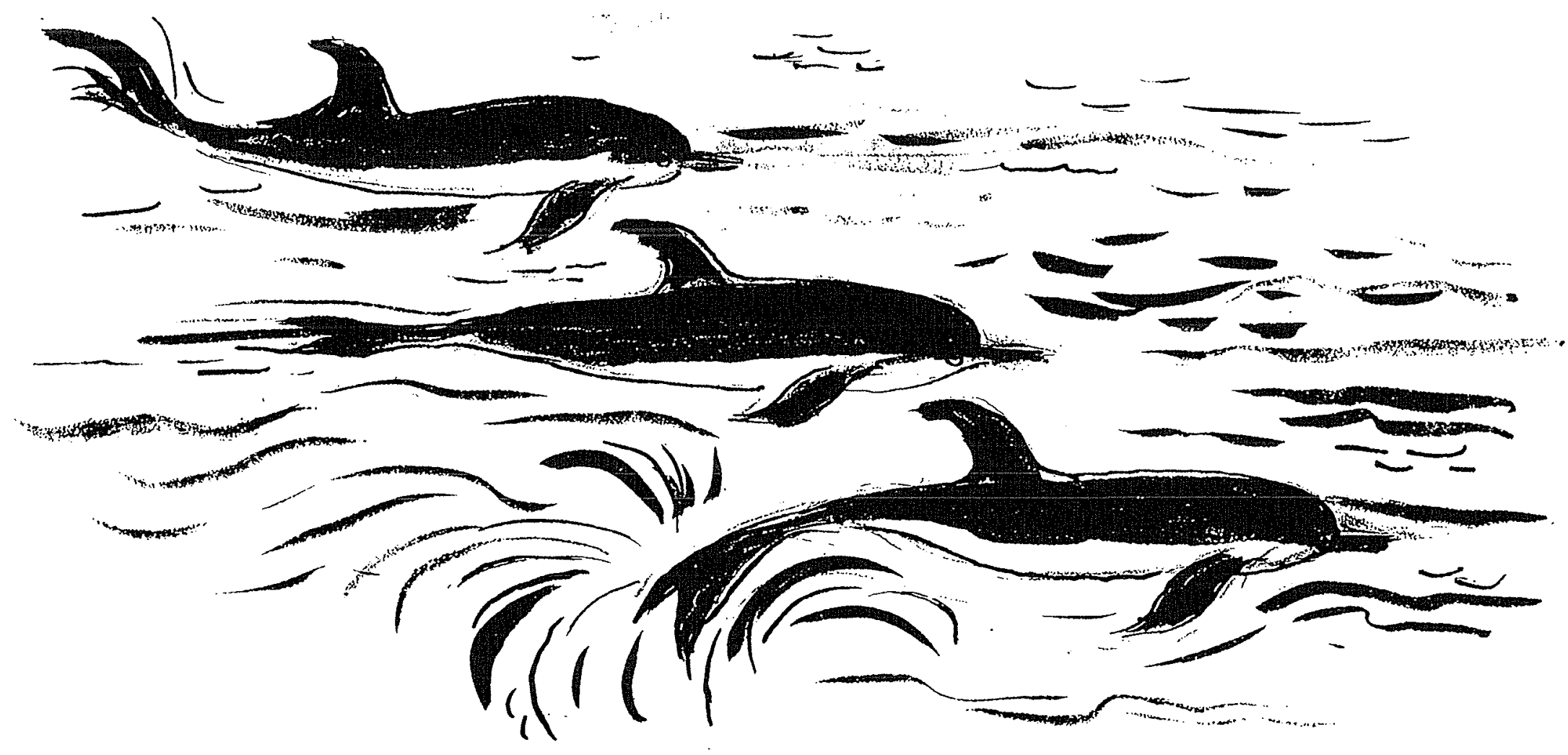

Figure 4.3 'Kerplunk' as it would look to an observer looking across the surface of the water. The three drawings show the chronological progression of the behavior (top to botiom). 


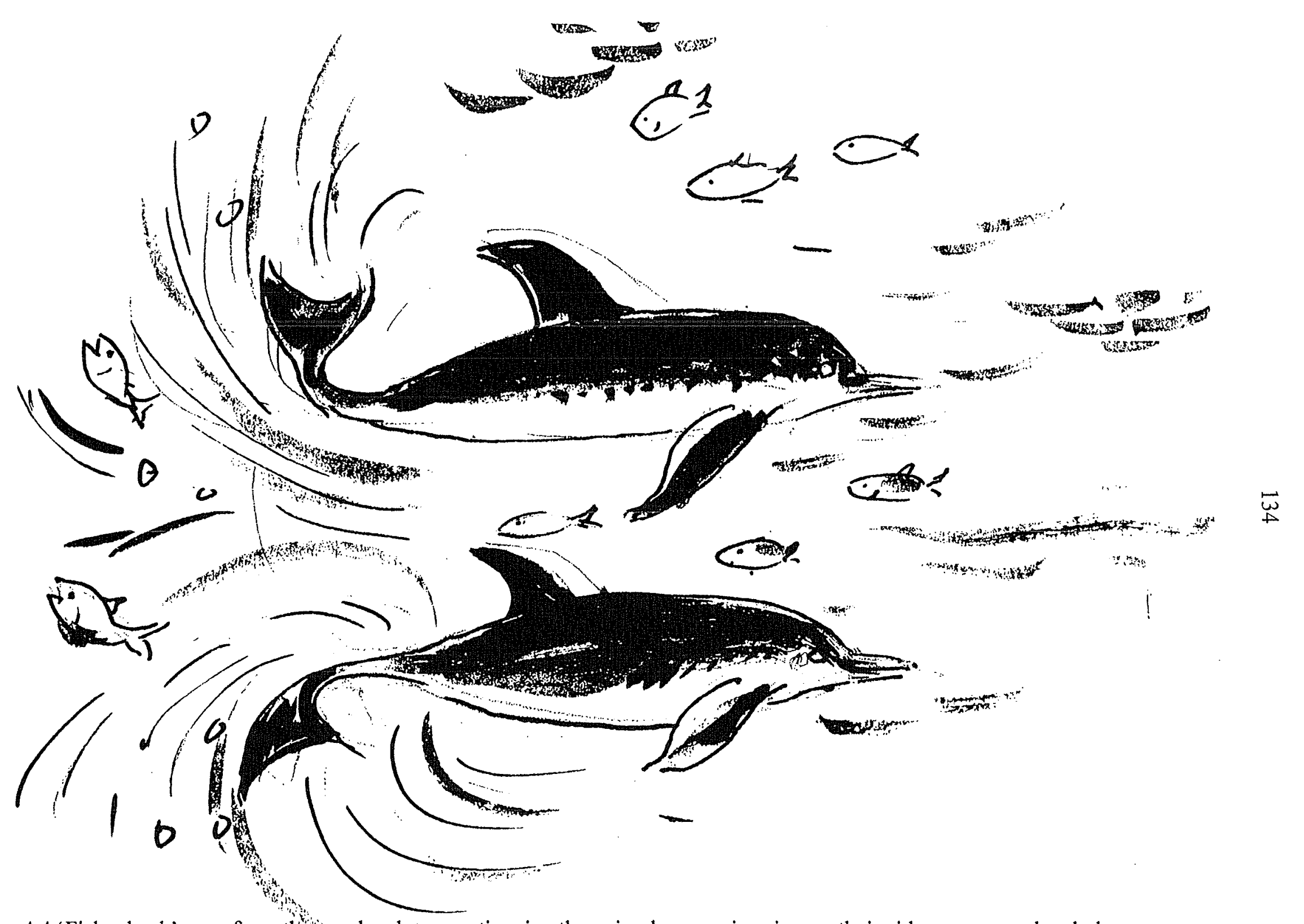

Figure 4.4 'Fish whack' seen from the 'averhead perspective, i.e. the animals are swimming on their sides so an overhead observer sees a lateral view of the animals. As shown, the whack can be either a dorsal or ventral thrust of the flukes. 


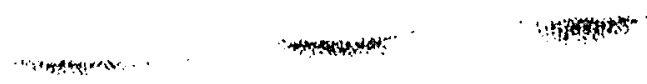

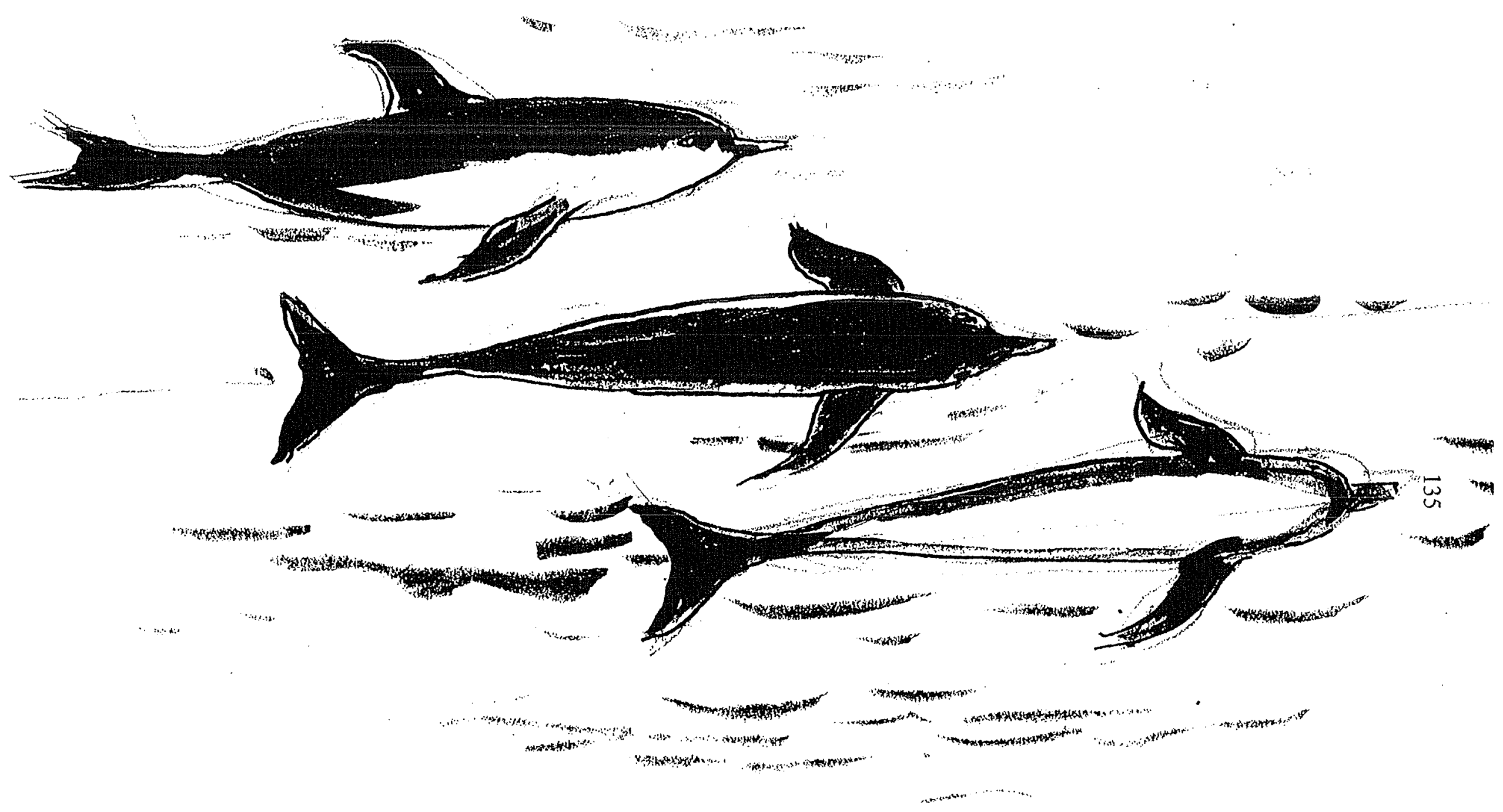

Figure 4.5 'Side-swimming' as seen from the overhead perspective (top drawing) and from the perspective of an observer underwater at the same depth as the animal (bottom two drawings). 


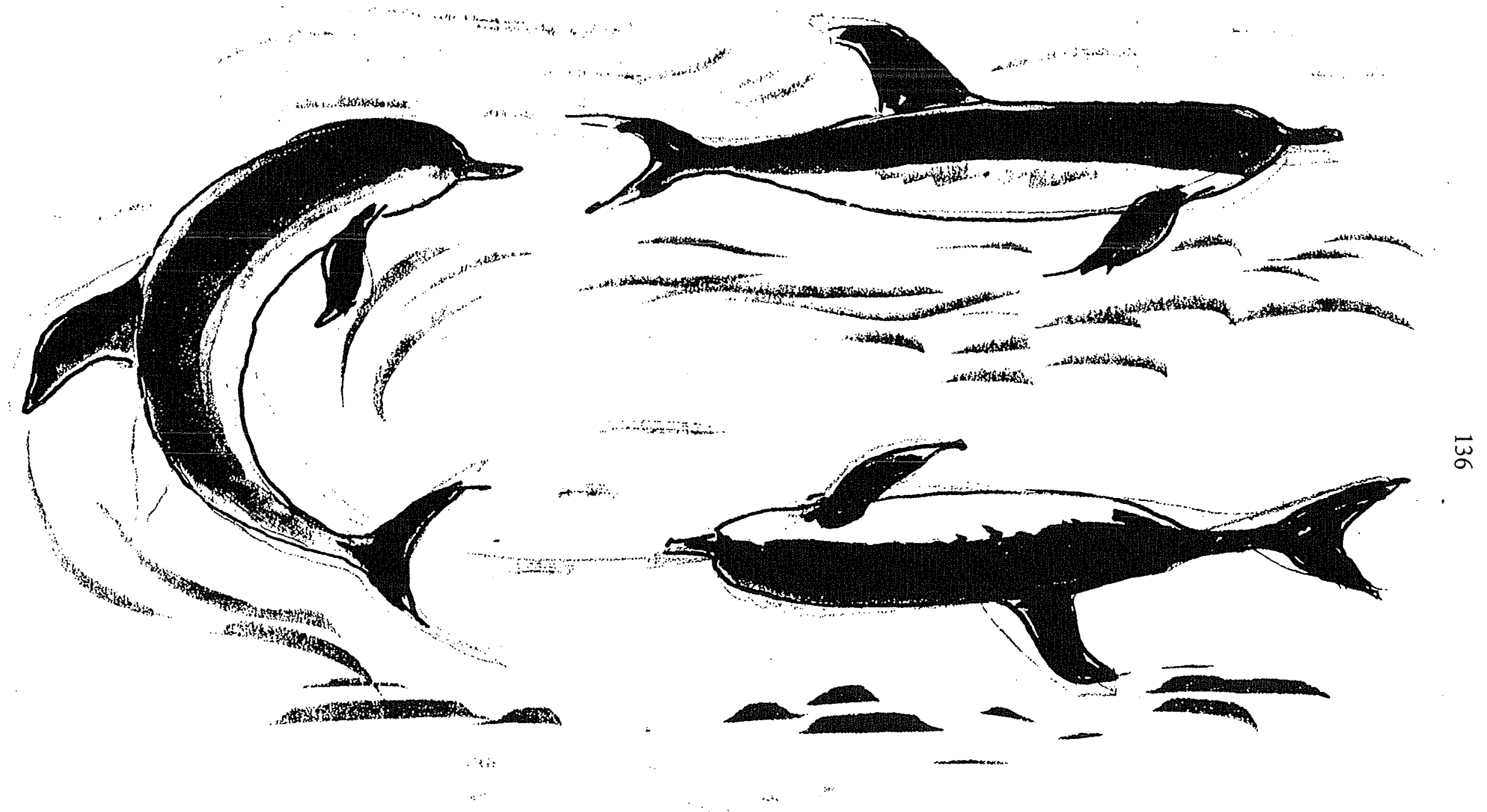

Figure 4.6 'Pinwheel' as seen from overhead. The key to visualizing this behavior is to understand that in the figure all three drawings . show the right side of the animal. From overhead, the same lateral side of the animal is in view throughout the behavior. 


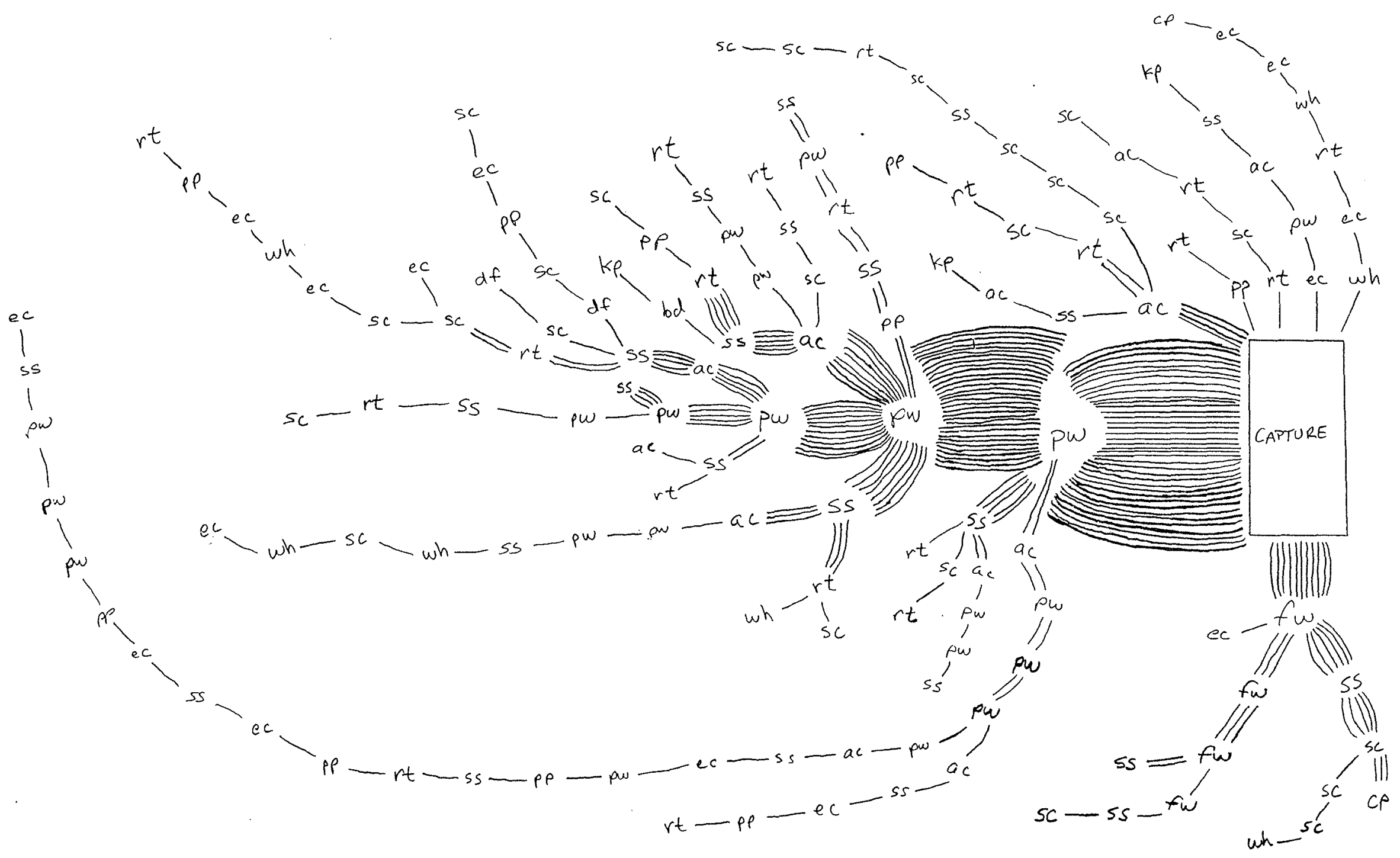

Figure 4.7. Behavioral sequences leading to successful captures. Each transition represents a single state step between behaviors. Sequences were traced backwards to either non-foraging behavior or another capture event. Abbreviations are as follows: cp-capture; pw-pinwheel; ac-accelerate; fw-fish whack; rt-root; ss-side swim; kp-kerplunk; sc-scan; ec-echolocate; wh-whistle; pp-pop; df-drift; bd-bottom disturbance/bubble cloud. 


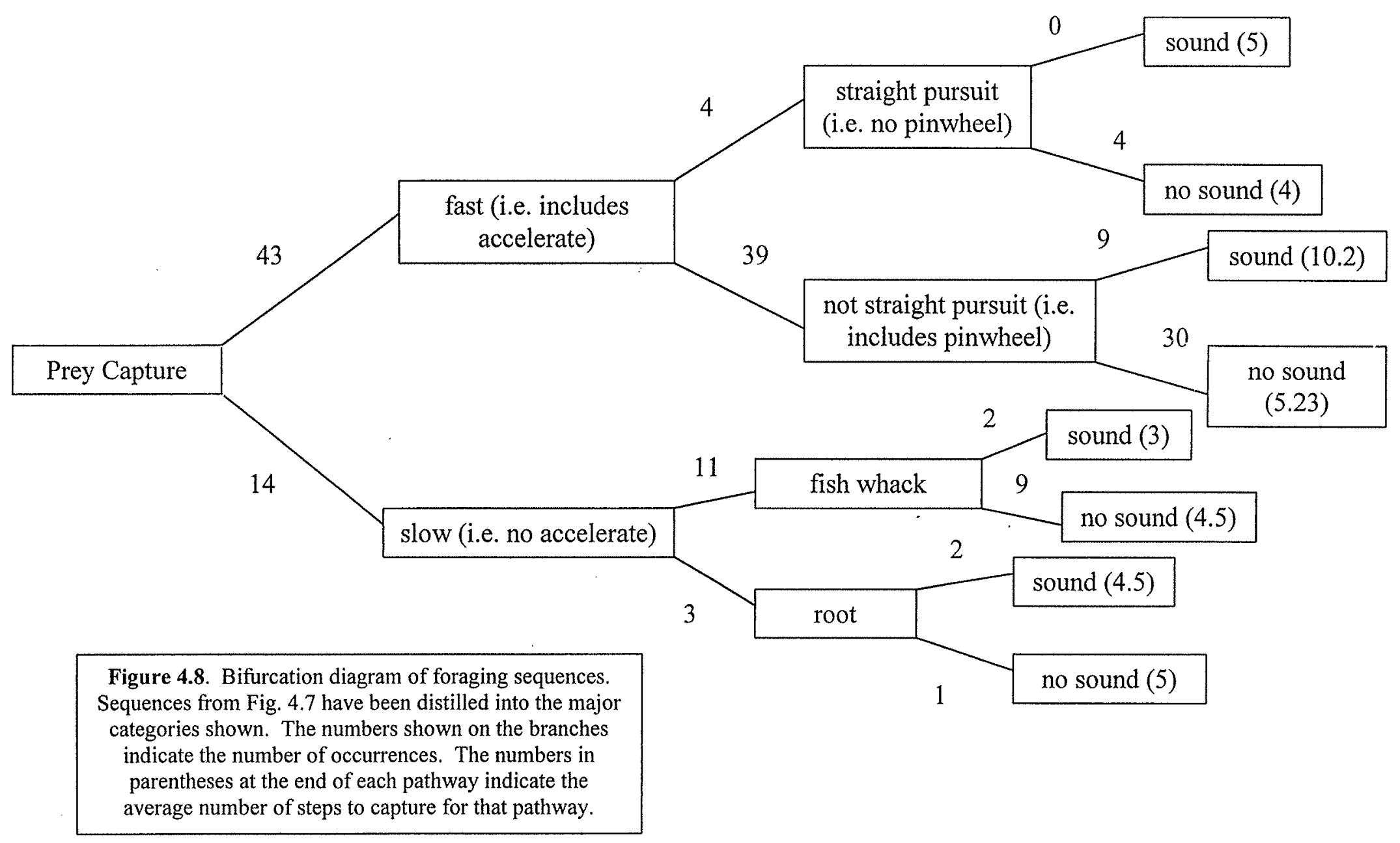




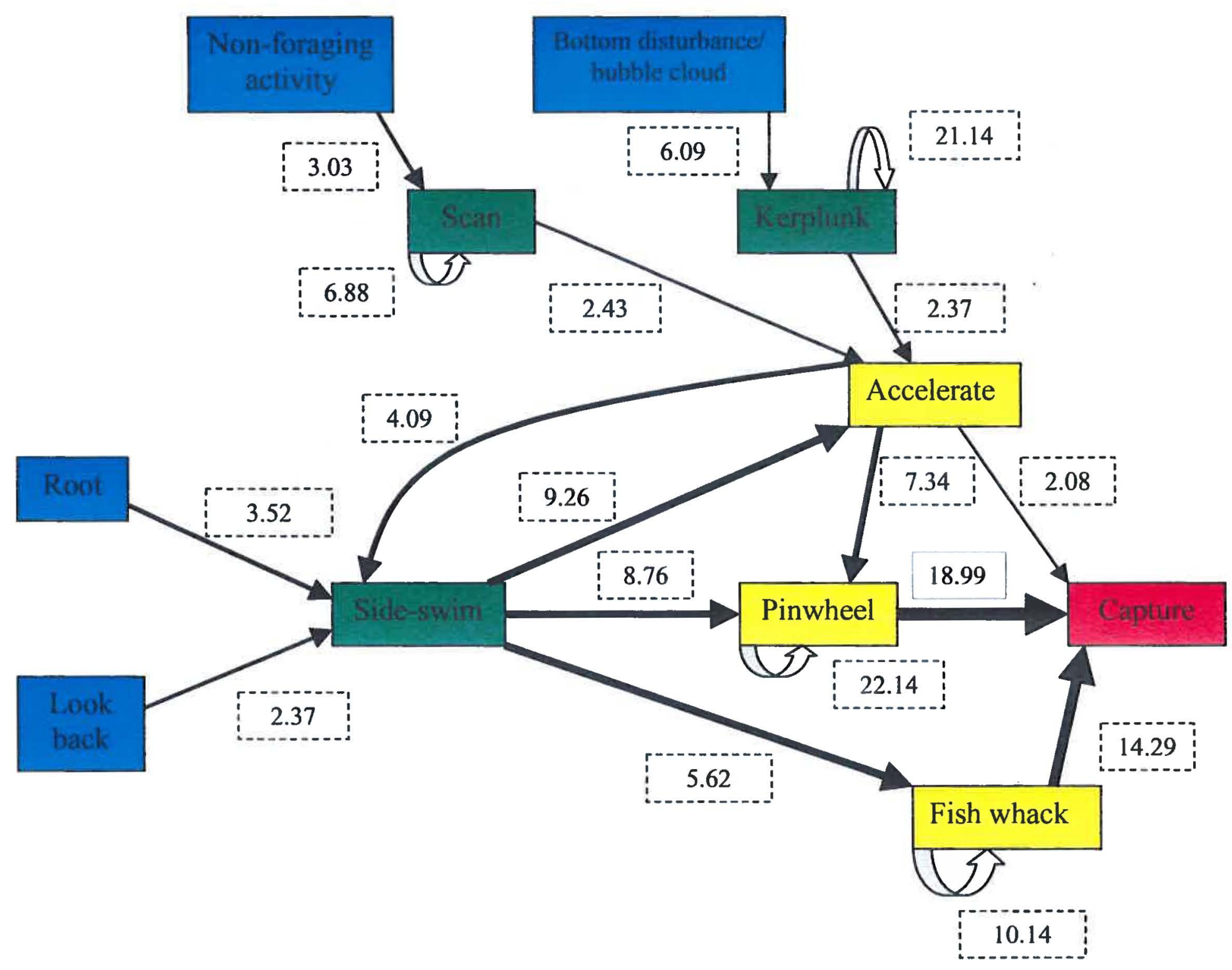

Figure 4.9 Behavioral Transitions Leading to Prey Capture: State-lag Z-scores. The different colored boxes for the various behaviors (Figures 4.7-10) are meant to group behaviors relative to their minimum number of steps (i.e. transitions) away from capture. 


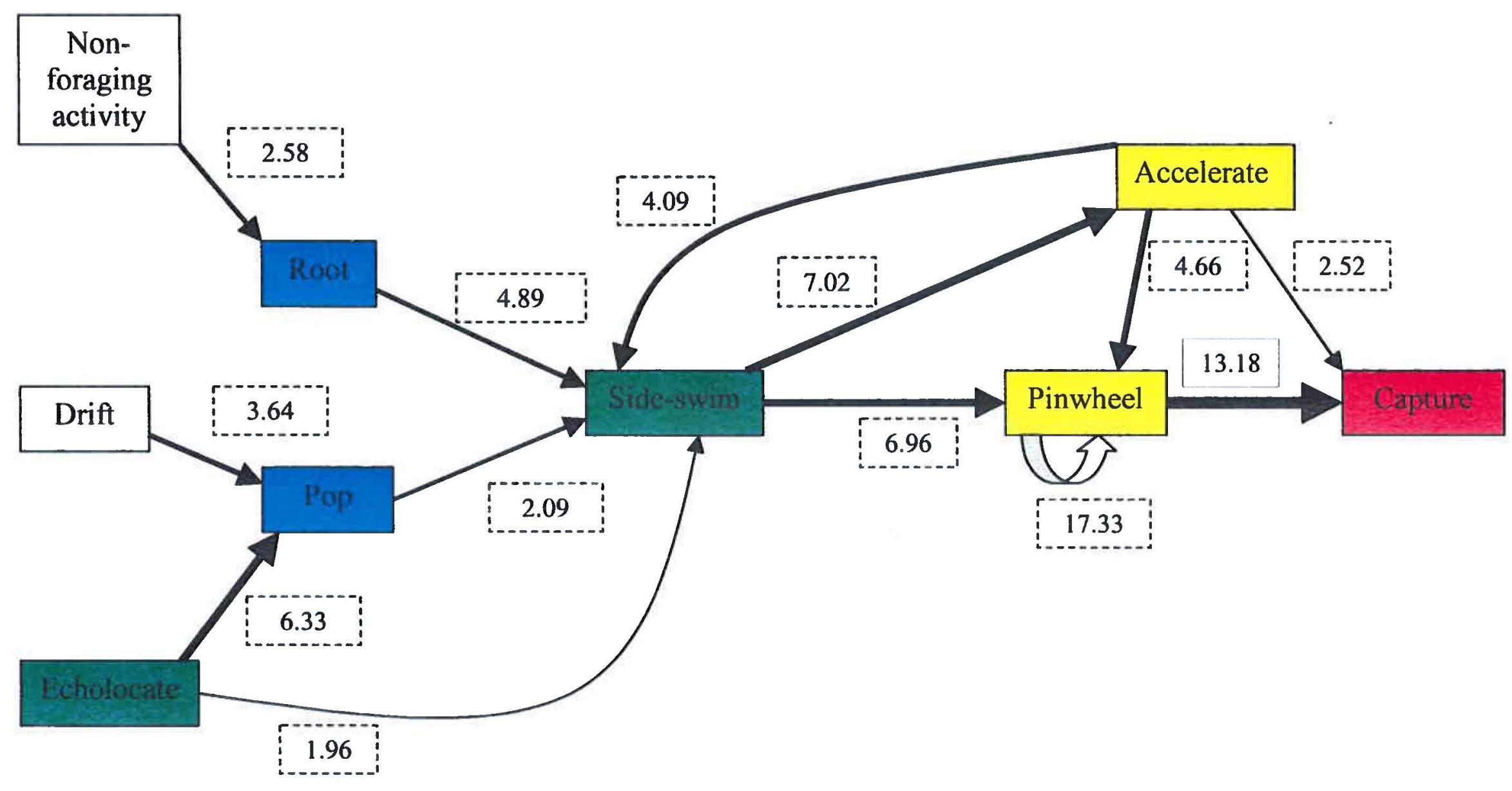

Figure 4.10 Behavioral Transitions Leading to Prey Capture: State-lag Z-scores for Sand Feeding 


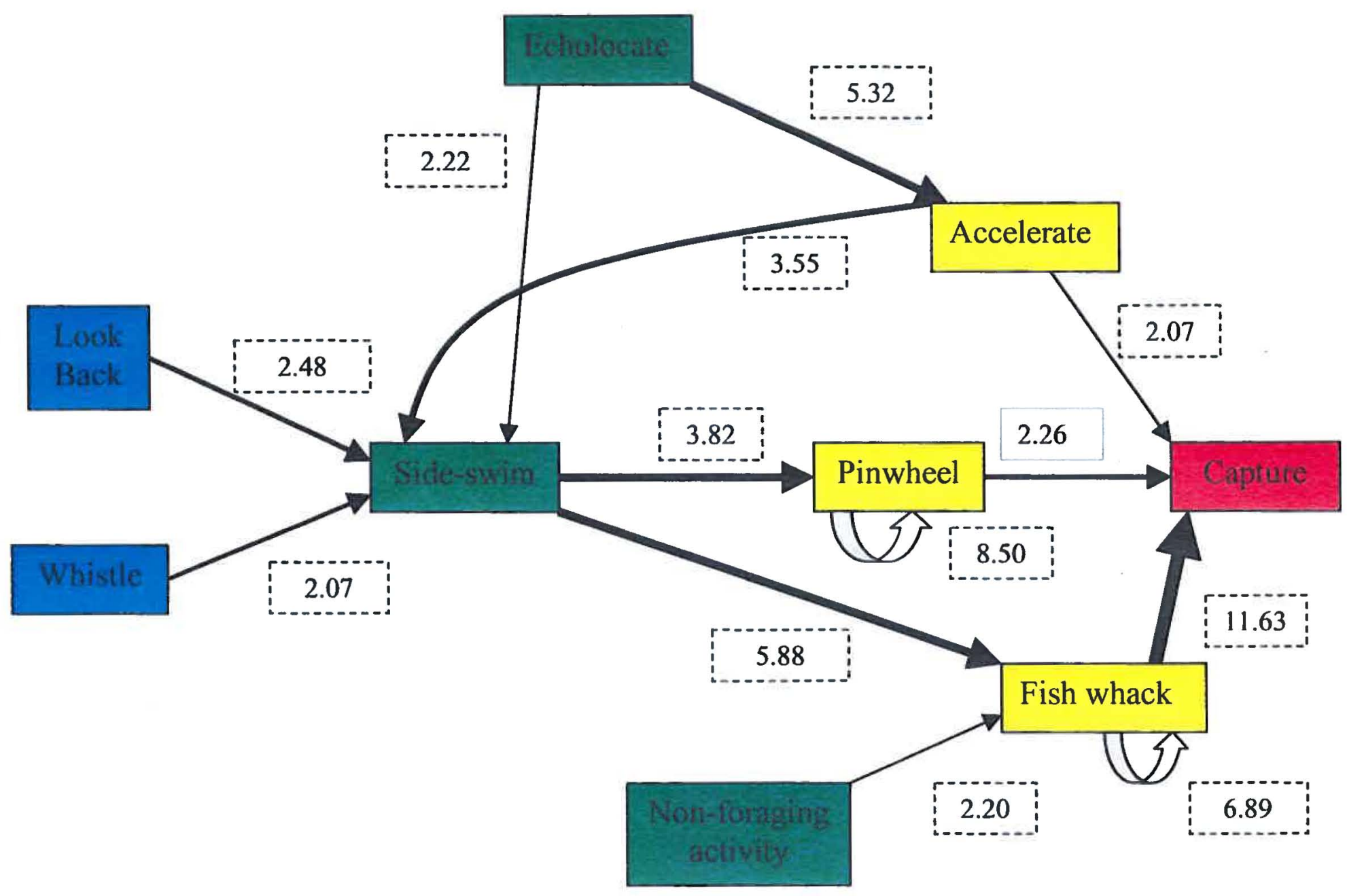

Figure 4.11 Behavioral Transitions Leading to Prey Capture: State-lag Z-scores Seagrass Feeding 


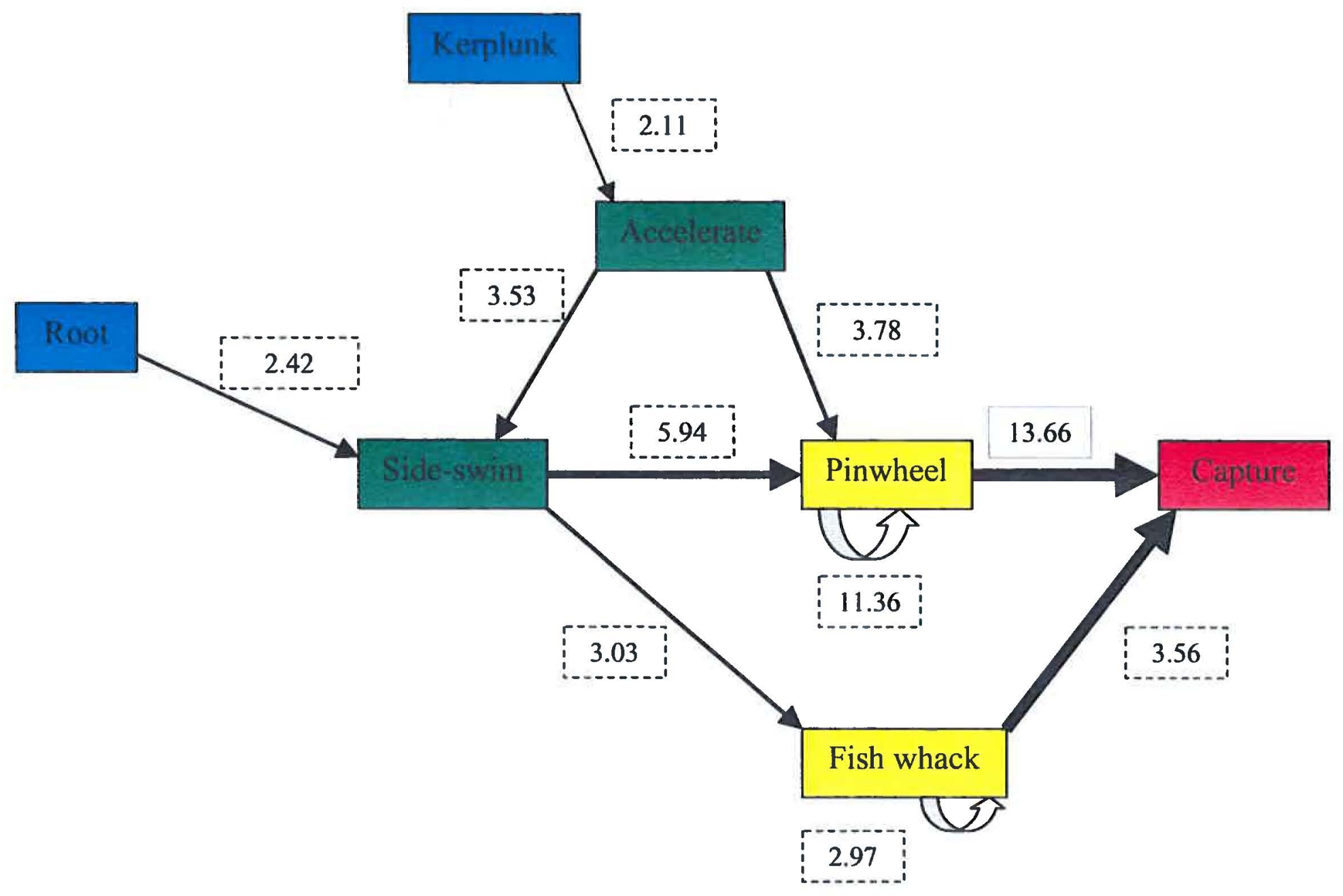

Figure 4.12 Behavioral Transitions Leading to Prey Capture: State-lag Z-scores for Seagrass Edge Feeding 


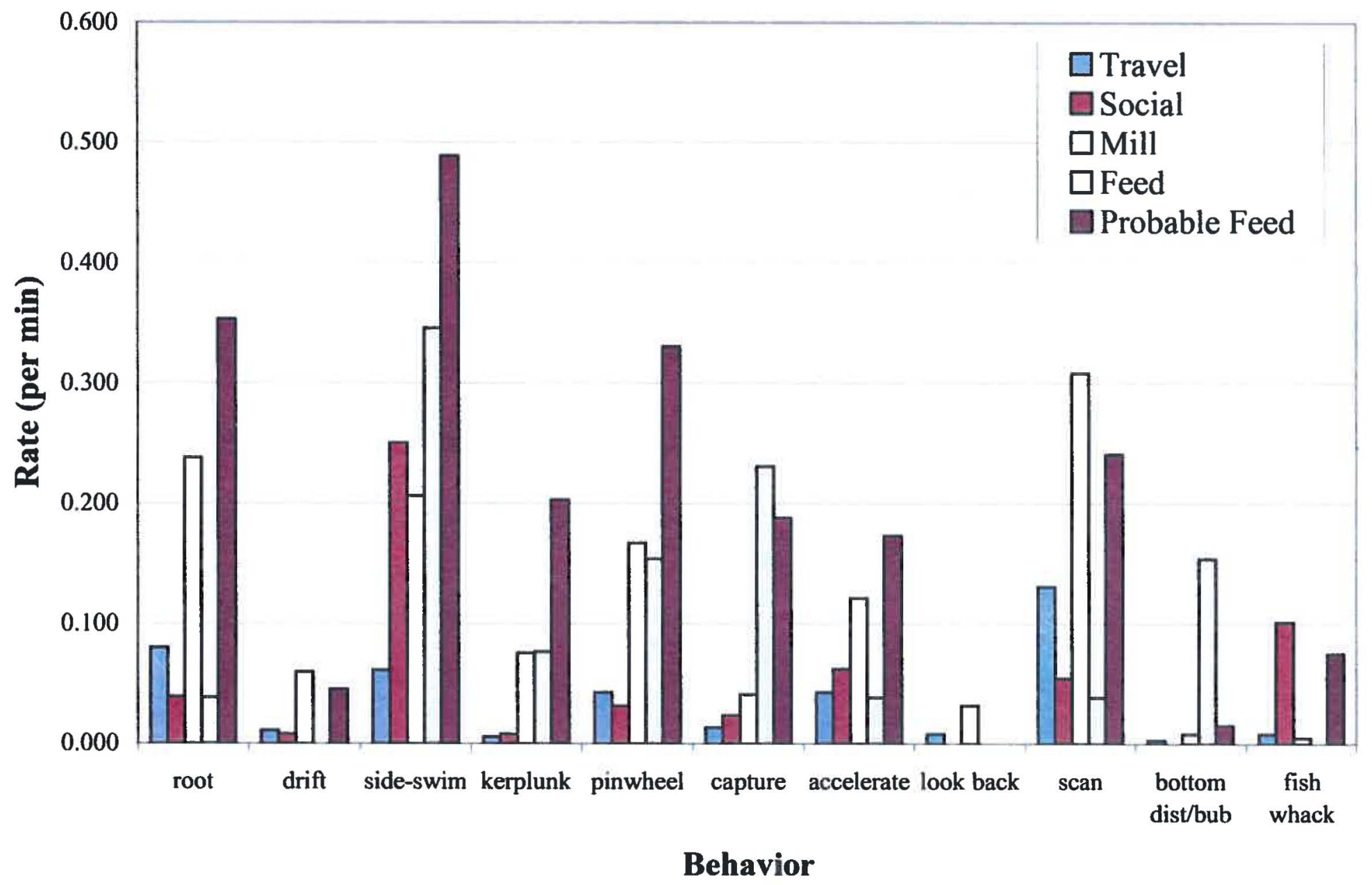

Figure 4.13 Rate of occurrence of foraging behaviors during general activities. 


\subsection{Literature Cited}

Altmann, J. (1974). Observational study of behavior: sampling methods. Behaviour 49: $227-267$.

Anderson, T. W. \& Goodman, L. A. (1957). Statistical inference about Markov chains. Annals of Mathematical Statistics 28: 89-110.

Au, W. W. L. (1993). The sonar of dolphins. Springer-Verlag, New York.

Bakeman, R. \& Gottman, J. M. (1986). Observing Interaction: an introduction to sequential analysis. Cambridge University Press, Cambridge.

Barros, N. B. (1992). Prey of bottlenose dolphins (Tursiops truncatus) resident in the Sarasota Bay area of the west coast of Florida, pp. 74. Contract Report for the Dolphin Biology Research Institute, Sarasota, FL, Sarasota, FL.

Barros, N. B. \& Odell, D. K. (1990). Food habits of bottlenose dolphins in the Southeastern United States. In The Bottlenose Dolphin (ed. S. Leatherwood and R. R. Reeves), pp. 309-328. Academic Press, San Diego.

Barros, N. B. \& Wells, R. S. (1998). Prey and feeding patterns of resident bottlenose dolphins (Tursiops truncatus) in Sarasota Bay, Florida. Joumal of Mammology 79: 1045-1059.

Bassos, M. K. (1993). A behavioral assessment of the reintroduction of two bottlenose dolphins. Master of Science thesis, University of California, Santa Cruz.

Beauchamp, G., Giraldeau, L. A. \& Ennis, N. (1997). Experimental evidence for the maintenance of foraging specializations by frequency-dependent choice in flocks of spice finches. Ethology, Ecology, and Evolution 9: 105-117.

Blaxter, J. H. S., Gray, J. A. B. \& Denton, E. J. (1981). Sound and startle responses in herring shoals. Journal of the Biological Association of the UK 61: 851-869.

Blaxter, J. H. S. \& Hoss, D. E. (1981). Startle response in herring: the effect of sound stimulus frequency, size of fish, and selective interference with the acousticolateralis system. Journal of the Biological Association of the UK 61: 871-879.

Caldwell, D. K. \& Caldwell, M. C. (1972). The World of the Bottlenose Dolphin. J. B. Lippincott, Philadelphia. 
Caldwell, D. K., Caldwell, M. C. \& Evans, W. E. (1966). Sounds and behavior of captive Amazon fresh-water dolphins, Inia geoffrensis. Los Angeles City Museum, Contributions Scientific 108: 24.

Caro, T. M. \& Hauser, M. D. (1992). Is there teaching in nonhuman animals? Quarterly Review of Biology 67: 151-174.

Clarke, J. L., Jones, M. E. \& Jarman, P. J. (1995). Diurnal and nocturnal grouping and foraging behaviours of free-ranging eastern grey kangaroos. Australian Journal of Zoology 43: 519-529.

Connor, R. C., Smolker, R. A. \& Richards, A. F. (1992). Two levels of alliance formation among male bottlenose dolphins (Tursiops sp.). Proceedings of the National Academy of Sciences USA 89: 987-990.

Craig, W. (1918). Appetites and aversions as constituents of instincts. Biological Bulletin 34: 91-107.

Eaton, R. C., Bombardieri, R. A. \& Meyer, D. L. (1977). The mauthner-initiated startle response in teleost fish. Journal of Experimental Biology 66: 65-81.

Evans, R. M. (1982). Efficient use of food patches at different distances from a breeding colony in black-billed gulls. Behaviour 79: 28-38.

Everitt, B. S. (1977). The Analysis of Contingency Tables. Chapman and Hall, London.

Fay, R. R. (1988). Hearing in Vertebrates: a Psychophysics Databook. Hill-Fay, Winnetka, IL.

Felleman, F. L., Heimlich-Boran, J. R. \& Osborne, R. W. (1991). The feeding ecology of killer whales (Orcinus orca) in the pacific northwest. In Dolphin Societies: Discoveries and Puzzles (ed. K. Pryor and K. S. Norris), pp. 113-147. University of California Press, Berkeley, CA.

Galef, B. G., Jr. (1995). Why behaviour patterns that animals learn socially are locally adaptive. Animal Behaviour 49: 1325-1334.

Goss-Custard, J. D. \& Le V dit Durell, S. E. A. (1983). Individual and age differences in the feeding ecology of oystercatchers Haematopus ostralegus wintering on the Exe Estuary, Devon. Ibis 125: 155-171.

Goss-Custard, J. D., Le V dit Durell, S. E. A. \& Ens, B. J. (1982). Individual differences in aggressiveness and food stealing among wintering oystercatchers, Haematopus ostralegus. Animal Behavior 30: 917-928. 
Goss-Custard, J. D. \& Sutherland, W. J. (1984). Feeding specializations in oystercatchers, Haepmatopus ostralegus. Animal Behavior 32: 299-301.

Haenel, N. J. (1986). General notes on the behavioral ontogeny of Puget Sound killer whales and the occurrence of allomaternal behavior. In Behavioral Biology of Killer Whales (ed. B. C. Kirkevold and J. S. Lockard), pp. 285-300. Alan R. Liss, Inc., New York.

Hayes, H. E., Winn, H. E. \& Petricig, R. (1985). Anomalous feeding behavior of a humpback whale. Journal of Mammalogy 66: 819-821.

Hoelzel, A. R., Dorsey, E. M. \& Stern, S. J. (1989). The foraging specializations of individual minke whales. Animal Behavior 38: 786-794.

Hoese, H. D. (1971). Dolphins feeding out of water in a salt marsh. Journal of Mammology 52: 222-223.

Irvine, A. B., Scott, M. D., Wells, R. S. \& Kaufmann, J. H. (1981). Movements and activities of the Atlantic bottlenose dolphin, Tursiops truncatus, near Sarasota, Florida. Fishery Bulletin 79: 671-688.

Jurasz, C. M. \& Jurasz, V. P. (1979). Feeding modes of the humpback whale, Megaptera novaeangliae, in southeast Alaska, pp. 69-83. Whales Research Institute, Tokyo.

Kalcounis, M. C. \& Brigham, R. M. (1995). Intraspecific variation in wing loading affects habitat use by little brown bats (Myotis lucifugus). Canadian Journal of Zoology 73: 89-95.

Kalko, E. K. V. (1995). Insect pursuit, prey capture and echolocation in pipistrelle bats (Microchiroptera). Animal Behaviour 50: 861-880.

Kodha, M. (1994). Individual specialized foraging repertoires in the piscivorous cichlid fish, Lepidiolamprologus profundicola. Animal Behavior 48: 1123-1131.

Le V. dit Durell, S. E. A. \& Goss-Custard, J. D. (1984). Prey selection within a size-class of mussels, Mytilus edulis, by oystercatchers, Haematopus ostralegus. Animal Behavior 32: 1197-1203.

Leatherwood, S. (1975). Some observations of feeding behavior of bottle-nosed dolphins (Tursiops truncatus) in the northern Gulf of Mexico and (Tursiops cf $T$. gilli) off southern California, Baja California, and Nayarit, Mexico. Marine Fisheries Review 37: 10-16. 
Lopez, J. C. \& Lopez, D. (1985). Killer whales (Orcinus orca) of Patagonia, and their behavior of intentional stranding while hunting near shore. Journal of Mammalogy 66: 181-183.

Mann, J. (1999). Behavioral sampling methods for cetaceans: a review and critique. Marine Mammal Science 15: 102-122.

Maynard Smith, J. (1982). Do animals convey information about their intentions? Journal of Theoretical Biology 97: 1-5.

McLaughlin, R. L., Grant, J. W. A. \& Kramer, D. L. (1992). Individual variation and alternative patterns of foraging movements in recently-emerged brook char (Salvelinus fontinalis). Behaviour 120: 286-300.

Moors, T. L. (1997). Is 'menage a trois' behavior important in mating systems? Behavioral patterns of breeding female bottlenose dolphins. Master of Science thesis, University of California Santa Cruz.

Nakamichi, M., Kato, E., Kojima, Y. \& Itoigawa, N. (1998). Carrying and washing of grass roots by free-ranging Japanese macaques at Katsuyama. Folia Primatologica 69: 35-40.

Norton-Griffiths. (1967). Some ecological aspects of the feeding behaviour of the oystercatcher Haematopus ostralegus on the edible mussel Mytilus edulis. Ibis 109: $412-424$.

Nowacek, D. P., Lange, W. N., Wells, R. S. \& Tyack, P. L. (1995). A new method for studying cetacean behavior and acoustics: overhead video combined with underwater audio. In Eleventh biennial conference on the biology of marine mammals, Orlando, FL.

Partridge, L. \& Green, P. (1985). Intraspecific feeding specializations and population dynamics. In Behavioural Ecology (ed. R. M. Sibly and R. H. Smith), pp. 207226. Blackwell, Oxford.

Petricig, R. (1995). Bottlenose dolphins (Tursiops truncatus) in Bull Creek, South Carolina. Ph.D. thesis, University of Rhode Island.

Pilleri, G., Gihr, M. \& Kraus, C. (1970). Feeding Behavior of the Gangetic Dolphin, Plantanista gangetica, in Captivity. In Investigations on Cetacea, vol. II (ed. G. Pilleri), pp. 69-73. Benteli AG, Berne. 
Rogers, T. \& Bryden, M. M. (1995). Predation of adelie penguins (Pygoscelis adeliae) by leopard seals (Hydrurga leptonyx) in Prydz Bay, Antarctica. Canadian Journal of Zoology 73: 1001-1004.

Rossbach, K. A. \& Herzing, D. L. (1997). Underwater observations of benthic-feeding bottlenose dolphins (Tursiops truncatus) near Grand Bahama Island, Bahamas. Marine Mammal Science 13: 498-504.

Savino, J. F. \& Stein, R. A. (1989). Behavior of fish predators and their prey: habitat choice between open water and dense vegetation. Environmental Biology of Fishes 24: 287-293.

Sayigh, L. S., Tyack, P. L. \& Wells, R. S. (1993). Recording underwater sounds of freeranging dolphins while underway in a small boat. Marine Mammal Science 9: 209-213.

Schmid-Hempel, P., Kacelnik, A. \& Houston, A. I. (1985). Honeybees maximize efficiency by not filling their crop. Behavioral Ecology and Sociobiology 17: 6166.

Shane, S. H. (1990). Behavior and ecology of the bottlenose dolphin at Sanibel Island, Florida. In The Bottlenose Dolphin (ed. S. Leatherwood and R. R. Reeves), pp. 245-265. Academic Press, San Diego.

Sharpe, F. A. \& Dill, L. M. (1997). The behavior of Pacific herring schools in response to artificial humpback whale bubbles. Canadian Journal of Zoology 75: 725-730.

Simmons, J. A., Fenton, M. B. \& O'Farrell, M. J. (1979). Echolocation and pursuit of prey by bats. Science 203: 16-21.

Sogard, S. M. \& Olla, B. L. (1993). The influence of predator presence on utilization of artificial seagrass habitats by juvenile walleye pollock, Theragra chalcogramma. Environmental Biology of Fishes 37: 57-65.

Stephens, D. W. \& Krebs, J. R. (1986). Foraging Theory. Princeton University Press, Princeton.

Sutherland, W. J. (1987). Why do animals specialize? Nature 325: 483-484.

Swennen, C., De Bruijn, L. L. M., Duiven, P., Leopold, M. F. \& Marteijn, E. C. L. (1983). Differences in bill form of the oystercatcher, Haematopus ostralegus; a dynamic adaptation to specific foraging techniques. Netherlands Journal of Sea Research 17: 57-83. 
Tinbergen, N. (1951). The Study of Instinct. Oxford University Press, Oxford.

Urian, K. W. \& Wells, R. S. (1996). Bottlenose dolphin photo-identification workshop, pp. 92. National Marine Fisheries Service, Southeast Fisheries Science Center, Charleston, SC.

Waples, D. M., Wells, R. S., Costa, D. P. \& Worthy, G. A. J. (1995). Seasonal changes in activity and habitat use by bottlenose dolphins (Tursiops truncatus) in Sarasota Bay, FL. In Eleventh Biennial Conference on the Biology of Marine Mammals, Orlando, FL.

Weinrich, M. T., Schilling, M. R. \& Belt, C. R. (1992). Evidence for acquisition of a novel feeding behaviour: lobtail feeding in humpback whales, Megaptera novaeangliae. Animal Behavior 44: 1059-1072.

Wells, R. S. (1991). The role of long-term study in understanding the social structure of a bottlenose dolphin community. In Dolphin Societies: Discoveries and Puzzles (ed. K. Pryor and K. S. Norris), pp. 199-225. University of California Press, Berkeley.

Wells, R. S., Irvine, A. B. \& Scott, M. D. (1980). The social ecology of inshore odontocetes. In Cetacean Behavior: Mechanisms and Functions (ed. L. M. Herman), pp. 263-317. John Wiley \& Sons, New York.

Wells, R. S. \& Scott, M. D. (1999). Bottlenose dolphin Tursiops truncatus (Montagu, 1821). In Handbook of Marine Mammals, the Second Book of Dolphins and Porpoises, vol. 6 (ed. S. H. Ridgway and R. Harrison), pp. 137-182. Academic Press, San Diego.

Wells, R. S., Scott, M. D. \& Irvine, A. B. (1987). The social structure of free-ranging bottlenose dolphins. In Current Mammalogy, vol. 1 (ed. H. H. Genoways), pp. 247-305. Plenum Press, New York.

Whiten, A. \& Ham, R. (1992). On the nature and evolution of imitation in the animal kingdom: reappraisal of a century of research. In Advances in the Study of Behavior, vol. 21 (ed. P. J. B. Slater, J. S. Rosenblatt, C. Beer and M. Milinski), pp. 239-283. Academic Press, New York.

Würsig, B. \& Würsig, M. (1980). Behavior and ecology of dusky dolphins, Lagenorhynchus obscurus, in the south atlantic. Fishery Bulletin 77: 871-890.

Young, D. D. \& Cockcroft, V. G. (1994). Diet of common dolphins (Delphinus delphis) off the south-east coast of southern Africa: opportunism or specialization? Journal of Zoology, London 234: 41-53. 
Zottoli, S. J. (1977). Correlation of the startle reflex and Mauthner cell auditory responses in unrestrained goldfish. Journal of Experimental Biology 66: 243-254. 


\section{Chapter 5. Ambient Acoustics of Foraging Bottlenose Dolphins}

\subsection{Introduction}

Bottlenose dolphins inhabit an acoustically diverse environment with sounds produced by their prey (Barros \& Wells, 1998; Fish \& Mowbray, 1970), anthropogenic noise, other biological non-prey sources (e.g. shrimp), as well as the sounds they produce. Traditionally three different types of sounds have been attributed to bottlenose dolphins: whistles, echolocation clicks, and burst-pulse sounds (Popper, 1980). Whistles are frequency modulated tonal sounds with fundamental frequencies ranging from $2-20 \mathrm{kHz}$ (Caldwell \& Caldwell, 1965). Echolocation clicks are short $(50-150 \mu \mathrm{sec})$ broadband pulses with center frequencies between $65-120 \mathrm{kHz}$ and $-3 \mathrm{~dB}$ bandwidth of approximately $40 \mathrm{kHz}$ ( $\mathrm{Au}, 1993)$. Burst-pulse sounds encompass a wide variety of sounds that are highly variable in structure and length (Caldwell \& Caldwell, 1967a; Wood, 1953). The functions of bottlenose dolphin vocalizations have been the subject of considerable research. The commonly held assumption is that whistles and burst-pulse sounds are used for social communication and echolocation is used for food finding, predator avoidance, and navigation. These narrow assignments have been challenged (Amundin, 1991; Tyack, 1997) including reports of a social function for sounds that are echolocation-like in being broadband and impulsive but they are longer (msec vs. $\mu \mathrm{sec}$ scale) with energy concentrated in lower frequencies (Caldwell, Haughen \& Caldwell, 1962; Connor \& Smolker, 1996; dos Santos et al., 1990; McCowan \& Reiss, 1995). To test for the function of a particular sound, the behavioral context of its production must be understood. The behavioral precursor or response could involve both the signaling animal and a receiver in the case of a socially relevant signal, or just the signaler if the function is for individual food finding or navigation.

In some cases, however, signals used for finding food may not involve only the signaler but also the prey. Echolocating bats, for example, must contend with prey that 
can detect their sonar sounds (Roeder \& Treat, 1957; Roeder, Treat \& Vande Berg, 1968) and in some cases even possess behavioral avoidance measures (e.g. reflexive diving maneuvers) activated by a bat echolocation signal (Miller, 1983; Roeder, 1962; Roeder, 1967). At least somewhat in response to these abilities bats have adopted both behavioral and acoustic countermeasures. One behavioral adaptation to evasive maneuvers of insects consists of using the tail membrane to increase the surface area available to capture an insect in random, reflexive flight (Simmons, Fenton \& O'Farrell, 1979). In an acoustic countermeasure some bats hunt without echolocation. Instead the bats detect and localize their prey using the sounds produced by the prey (Ryan \& Tuttle, 1983; Ryan, Tuttle \& Taft, 1981; Tuttle \& Ryan, 1981).

Bottlenose dolphins possess a sophisticated echolocation system that has been shown through captive experiments to be excellent for target detection, ranging, and discrimination (Au, 1993). Ecological constraints may select against the use of an active sonar system for prey detection. Most prey are considered to have poor hearing abilities in the range of dolphin echolocation because the sounds they produce are of substantially lower frequency (Fish \& Mowbray, 1970) and fish hearing abilities in the echolocation frequency range have seldom been tested (Fay, 1988). Broadband, high frequency sounds, however, have been effective in keeping fish away from intake pipes (Astrup \& Mfhl, 1993; Dunning et al., 1992), and $80 \mathrm{kHz}$ pure tone sounds, within the range of echolocation frequencies, elicit a startle response in shad (Mann, Lu \& Popper, 1997). This information, especially in light of the bat-insect arms race, indicates that the ecology of an active sensory system like dolphin echolocation may be more complex than the simple paradigm that dolphins echolocate to find food.

Whistles and burst-pulse sounds could have foraging functions in addition to social ones. The lower frequency nature of these sounds permits greater travel distances making them suitable for food calls or coordination in the case of cooperative foraging. Food calls have been identified for primate species (Clark \& Wrangham, 1994; Hauser \& Marler, 1993a; Hauser \& Marler, 1993b) and food-associated calls have been identified for dolphins (Janik, 1997). Additionally broadband, echolocation-like sounds are 
definitely not restricted to navigation and/or food finding. For example, a sound reported recently is a hybrid between an echolocation click and a burst-pulse sound. Connor and Smolker (1996) described a 'pop' as a broadband pulse rich in low frequency and longer than a typical echolocation click; this sound serves a social function in Shark Bay, Western Australia dolphins (Tursiops spp.). We are in the early stages of understanding the ecology of cetacean sound use and the present chapter explores the ecology of 'ambient' acoustics of foraging bottlenose dolphins.

\subsection{Materials and Methods}

A two-hydrophone towed system was used to record acoustic activity of freeranging bottlenose dolphins. This system was part of the integrated observation platform described in Appendix 1. Briefly, two hydrophones (HTI96) were towed from the observation vessel using the method described by Sayigh (1993). The cylindrically shaped hydrophone elements were modified to improve flow characteristics (i.e. reduce flow noise). Weights attached to the hydrophone cables kept the elements approximately $0.5 \mathrm{~m}$ below the surface when being towed at 2-3 knots. The audio signal from each hydrophone was filtered through a $4 \mathrm{kHz}$ high-pass filter (Allen Avionics 4188-4PO), and then recorded on the two audio channels of the video recorder (see Appendix 1). The high-pass filters were necessary to reduce flow noise while underway so that quality recordings of dolphin sounds could be obtained (i.e. the dynamic range of the recorder would not permit recording full flow noise and dolphin sounds).

Multi-looped whistles were scored as a single event, as was a train of echolocation clicks. The actual number of clicks could not be reliably counted due to the directional beam pattern and high frequency of dolphin echolocation. The system could record only the low frequency portion of echolocation clicks, but the main energy is in frequencies well above what the system could record. This shortcoming cannot necessarily be overcome with equipment capable of higher sampling rates. To access the main energy of the clicks requires not only higher sampling rates but also that the sensor must be within the narrow transmit beam pattern ( $\mathrm{Au}, 1993)$. Given the beam pattern restrictions, the added complexity of incorporating high sampling rate equipment was not 
attempted. Under some circumstances it was likely that all clicks in a train were recorded, and the overhead video often showed the animal's orientation to one or both hydrophones. For two reasons, however, even knowing the angle of the animal relative to the sensor does not permit calculation of expected energy, which could indicate the likelihood of recording every click. First, neither the sound pressure level (SPL) nor spectral content of clicks at off-axis (i.e. $>20^{\circ}$ ) angles is known, and secondly bottlenose dolphins are known to vary the source level (Au, 1993). Harbor porpoises, Phocoena phocoena, may even vary the beam pattern (Amundin, 1991) of their clicks. For all of these reasons a train of echolocation clicks was scored as a single event, and given these constraints echolocation may be underrepresented in these 'ambient' recordings. A pause of $\geq 1$ second was interpreted as the end of a click train. Records of other sounds should represent the total number of sounds produced. The other vocalizations considered here have energy concentrated in relatively low frequencies (see below) and are considered to be less directional, therefore increasing the likelihood of their being recorded. The sounds considered in this chapter are referred to as 'ambient' because with this system the source of a sound could not be identified to a particular individual if more than one were in the immediate area $(<1000 \mathrm{~m})$. In many cases, however, only one animal was within $1000 \mathrm{~m}$ of the observation vessel during the focal follow, hereafter referred to as a 'single animal'.

During tape review sounds were scored as events in The Observer ${ }^{\circledR}$ simultaneously with the focal dolphin's behavior so they could be analyzed within the context of observed behaviors (see Section 4.2). In addition, the focal animal's habitat was included as a modifier for each acoustic event to permit analyses of the influence of environment on sound use. Using this method, the vocalizations could be analyzed in the context of foraging as well as general activity state (e.g. traveling, socializing), nearest neighbor distance, and presence/absence of a calf.

As described above, the data collection system did not permit sound source localization, so for multi-animal groups it was not known which animal produced a sound. In the absence of complete sound localization data, calculation of exact individual 
animal vocal rate is impossible for multi-animal groups. The analyses conducted here account for this lack of information by not relying on vocal rates in multi-animal groups beyond a general, introductory analysis of sound production patterns. Instead, comparisons of sound use are done between single and multi-animal groups using primarily contingency table analyses because they permit comparisons that do not depend on individual rates of sound production, i.e. they compare the relative occurrence of the different sounds. Rates of sound production are used only for descriptive purposes and are, for multi-animal groups, average rates for a given group. For the contingency table analyses, however, the total numbers of sounds recorded for multi-animal groups were retained for two reasons. First, given that a sound could not be assigned to an individual it was possible that only one animal was responsible for all sounds recorded. The second null hypothesis is that each animal in a group is vocalizing in the same pattern as it would if it was alone. In either case the contingency tables would reveal predictable ratios of type of sound produced and single vs. multi-animal group. Analyses of foraging specific functions of sounds, e.g. habitat-specific use, consider only sounds recorded from single animals.

\subsection{Results}

Three types of sounds were scored as the overhead video/audio tapes from focal individuals (Table 5.1) were reviewed: 1) clicks; 2) whistles; and 3)'pops' (Figures 5.1ac). Pops have not been reported previously for the Sarasota bottlenose dolphins, and are somewhat similar to the sounds reported by other studies ('pops' (Connor \& Smolker, 1996); 'thunks' (McCowan \& Reiss, 1995); and 'low creaks' (dos Santos et al., 1990)). They differ in having more energy at higher frequencies and are longer in duration (see Figure 5.1c). Whistles recorded during observations were typical frequency modulated, tonal calls as have been reported previously for the Sarasota bottlenose dolphins (Sayigh et al., 1990; Sayigh et al., 1995).

The average rate of sound production per animal in multi-animal groups was significantly less than the rate for single animals over all behaviors and sound types (Figure 5.2a). Similarly, single foraging animals have a significantly higher sound production rate than for animals in multi-animal groups (Figure 5.2b). When animals are 


\begin{tabular}{|c|c|c|c|cc|}
\hline Focal & Birth & & Reproductive & \multicolumn{2}{|c|}{ Animal-Minutes Observed } \\
Animal & Year & Sex & Status & Votal & Video \\
\hline FB03 & 1989 & F & A & 284 & 84 \\
\hline FB11 & 1984 & F & A & 110 & 44 \\
\hline FB28 & 1965 & M & A & 196 & 88 \\
\hline FB38 & 1974 & M & A & 120 & 31 \\
\hline FB54 & 1971 & F & A & 105 & 47 \\
\hline FB65 & 1983 & F & A & 230 & 34 \\
\hline FB75 & 1974 & F & A & 120 & 65 \\
\hline FB79 & 1979 & F & A & 85 & 33 \\
\hline FB83 & 1950 & F & A & 110 & 41 \\
\hline FB92 & 1988 & M & SA & 125 & 114 \\
\hline F101 & 1990 & F & A & 115 & 31 \\
\hline F118 & 1992 & M & SA & 185 & 138 \\
\hline F138 & 1992 & M & SA & 135 & 44 \\
\hline F155 & 1990 & F & SA & 175 & 93 \\
\hline F157 & $?$ & F & A & 265 & 87 \\
\hline UNK2 & $?$ & $?$ & SA/A & 115 & 31 \\
\hline UNK3 & $?$ & $?$ & SA/A & 100 & 36 \\
\hline Non-30's & n/a & n/a & n/a & 879 & 255 \\
\hline TOTALS & & 10F, 5M & 4SA, 11A & 3454 & 1296 \\
\hline
\end{tabular}

Table 5.1 Focal animals included in the study. UNK2\&3 were unknown at the time of publication. 'Non-30's' refers to data collected on animals for whom $<30 \mathrm{~min}$ of useable video/audio data were collected. A=Adult, $\mathrm{SA}=$ Sub-adult.

not foraging the sound production rates are not significantly different $(0.20$ sounds $/ \mathrm{min}$ for single animals and 0.16 sounds/animal/min for multi-animal groups). The relatively constant increase in sound production rate per animal as the group size increases is similar to that observed in clicking sperm whales (Whitehead \& Weilgart, 1990).

\subsubsection{Effects of group size and behavior on sound use}

To further investigate this difference in single vs. multiple animal groups the analyses were broken down by the three sound types. The use patterns for these three sounds are significantly influenced by whether an individual is alone or in a group (Table 5.2 and Figure 5.3) and whether foraging or not (Table 5.3 and Figure 5.4). 


\begin{tabular}{|c|c|c|c|}
\hline & \multicolumn{3}{|c|}{ Sound Type } \\
\hline Group Size & Pops & Echolocation & Whistles \\
\hline Single & $70[7.59]$ & $109[4.69]$ & $36[-11.87]$ \\
Multiple & $76[-4.37]$ & $141[-2.70]$ & $155[6.84]$ \\
\hline
\end{tabular}

Table 5.2 Counts of the three sound types in single vs. multi-animal groups. Adjusted residuals are shown in [brackets].

A chi-square analysis reveals that use patterns depend significantly on whether an animal is alone or part of a multi-animal group $\left(\chi^{2}=39.75, \mathrm{P}<<0.01\right)$. Comparing adjusted residuals to the standard normal deviate (i.e. 1.96) can give insight into the contribution of individual contingency table cells to the $\chi^{2}$ statistic (Everitt, 1977). Adjusted residuals (Table 5.2) indicate that single animals produce many more pops and echolocation than expected while producing far fewer whistles than expected. For multiple animals the residuals suggest the exact opposite. Similar $\chi 2$ analyses conducted after normalizing the number of multi-animal sounds to a single animal equivalent resulted in even larger $\chi^{2}$ values.

In addition, sound production depends on whether the animal(s) is foraging or not (Table 5.3 and Figure 5.4). Chi-square analyses conducted for each sound type independently revealed highly significant differences for all three sound types (pop $\chi^{2}=13.48, \mathrm{df}=1, \mathrm{P}<0.01$; echolocation $\chi^{2}=46.66, \mathrm{df}=1, \mathrm{P}<<0.001$; whistle $\chi^{2}=44.10, \mathrm{df}=1$, $\mathrm{P}<<0.001$ ). Comparison of expected values for the individual $2 \times 2$ contingency tables compiled in Table 5.3 show a consistent trend in the contribution of each category to the $\chi^{2}$ value. Single animals produce fewer echolocation trains, whistles, and pops than expected when not foraging and more than expected when foraging. For multiple animal groups the reverse is true for all three sounds.

\begin{tabular}{|c|c|c|c|c|c|c|}
\hline \multirow{2}{*}{ Group Size } & \multicolumn{2}{|c|}{ Pops } & \multicolumn{2}{c|}{ Echolocation } & \multicolumn{2}{c|}{ Whistles } \\
\cline { 2 - 7 } & $\begin{array}{c}\text { Non- } \\
\text { foraging }\end{array}$ & Foraging & $\begin{array}{c}\text { Non- } \\
\text { foraging }\end{array}$ & Foraging & $\begin{array}{c}\text { Non- } \\
\text { foraging }\end{array}$ & Foraging \\
\hline Single & 6 & 64 & 10 & 99 & 4 & 32 \\
Multiple & {$[15.14]$} & {$[54.86]$} & {$[34.86]$} & {$[74.14]$} & {$[21.67]$} & {$[14.33]$} \\
& 26 & 52 & 69 & 69 & 117 & 48 \\
& {$[16.86]$} & {$[61.14]$} & {$[14]$} & {$[93.86]$} & {$[99.33]$} & {$[65.67]$} \\
\hline
\end{tabular}


Table 5.3. Occurrence of sounds in single vs. groups of animals by behavior. 'Foraging' includes only those sounds recorded during previously defined foraging behaviors. Expected values are shown in [brackets].

\subsubsection{Habitat-specific use of sound during foraging}

Elucidating the ecology of sound function is also aided by an understanding of the habitat-specific use of particular sounds. Overall sound use is significantly influenced by habitat (Table 5.4, $\chi^{2}=48.0, \mathrm{P}<<0.001$ ). To investigate the influence of habitat on

\begin{tabular}{|c|ccccc|}
\hline \multirow{2}{*}{ Sound } & \multicolumn{4}{|c|}{ Habitat } \\
\cline { 2 - 5 } & Sand & Seagrass & Seagrass edge & Bay \\
\hline Pop & 90 & 3 & 38 & 5 \\
Echolocation & 115 & 19 & 62 & 35 \\
Whistle & 82 & 18 & 32 & 52 \\
\hline Totals & 287 & 40 & 132 & 92 \\
\hline
\end{tabular}

Table 5.4 Habitat-specific sound use across all behaviors. Numbers are counts of sounds recorded.

sound use during foraging only the sounds produced by a single animal foraging in the three primary habitats are considered (Table 5.5 and Figure 5.5). The use of these three sounds was significantly affected by the habitat in which the animal foraged $\left(\chi^{2}=9.89\right.$, $\mathrm{df}=4, \mathrm{P}<0.05$ ). Further analyses revealed significant differences in the use of particular sounds in specific habitats (Figure 5.5). Single foraging animals produced significantly more whistles in seagrass meadows $\left(\chi^{2}=5.009, \mathrm{df}=1, \mathrm{P}<0.05\right)$ and pops at the seagrass edge $\left(\chi^{2}=4.267, \mathrm{df}=1, \mathrm{P}<0.05\right)$ than expected.

\begin{tabular}{|c|c|c|c|}
\hline & \multicolumn{3}{|c|}{ Habitat } \\
\hline Sound & Sand & Seagrass & Seagrass edge \\
\hline Pop & 31 & 5 & $30^{*}$ \\
Echolocate & 56 & 12 & 28 \\
Whistle & 13 & $8^{*}$ & 11 \\
\hline
\end{tabular}

Table 5.5 Habitat specific sound use (numbers of sounds recorded) by a foraging single animal. See Chapter 4 for the description of habitats. * indicates sounds that were significantly more common than expected $(\mathrm{P}<0.05)$. 


\subsection{Discussion}

\subsubsection{Use of Sounds by Single vs. Multiple Animal Groups}

Single animals echolocate more than expected when foraging and less than expected when not foraging (Table 5.3, Figure 5.4). Echolocation has typically been associated with navigation and food finding. The present results combined with the sequential behavioral analyses (Section 4.6) support the hypothesis that echolocation is used during foraging, but the low rate of echolocation during single-animal travel $(0.04 / \mathrm{min})$ indicates that a dolphin's reliance on echolocation for navigation during the day seems less important than previously assumed. The goal of this study was to investigate the role of sound, specifically echolocation, in dolphin foraging. Figures 5.3 and 5.4 illustrate that echolocation is crucially important to a solitary foraging dolphin when compared to its use of echolocation when not foraging, which is perhaps surprisingly low. In addition, Figure 4.7 demonstrates that echolocation trains occur throughout foraging sequences. Given the constraints of recording these sounds in the wild these results may even underestimate the occurrence of echolocation during foraging.

Sound use varies significantly depending on whether an animal is alone or part of a group, and is manifest in both overall rates of vocalization and in the type of sound produced. The results indicate that in the presence of other animals the pattern of sound use is different than when an animal is alone. Sound use also varies significantly depending on whether a dolphin is foraging or not, and the significantly higher sound production rate for single foraging animals accounts for the observed differences in overall sound production rate. In the case of the three $2 \times 2$ tables compiled in Table 5.3 direct comparison of observed and expected values simplifies interpretation of observed patterns; adjusted residuals assist in interpretation by permitting comparisons of the contribution of an individual cell to the $\chi^{2}$ value. 
Sarasota dolphins may navigate using a variety of senses and information available to them. As year-round, long-term (4 generations) residents of the Sarasota Bay waters, these animals could be familiar with a variety of features of their environment. For example, bathymetric features such as channel confluences, subsurface rises, harbors, or even bridge pilings may provide navigational aids for Sarasota dolphins. Bats cease to echolocate after becoming familiar with an area, and will even avoid objects that have been removed or collide with newly introduced obstacles (Griffin, 1958; Holler, 1995; Neuweiler \& Mohres, 1966). Reduced reliance on echolocation for navigation would not be surprising if dolphins possess similar spatial memory capabilities. Other acoustic cues may also assist in navigation, e.g. bridge noise, fish aggregation areas.

For multi-animal groups the trend is opposite relative to single animals, i.e. they echolocate more than expected when not foraging and less than expected when foraging. Tyack (1997) presents arguments for the expansion of the traditional niches to which cetacean sounds have been assigned. In the case of echolocation, for example, he describes a model that could explain why dolphins foraging in groups might be able to successfully echolocate with reduced rates of click production. Tyack's (1997) model for the use of bistatic sonar in cetaceans describes a system in which an animal could use the information contained in a signal produced by a nearby conspecific. For example, if a fish is between the two dolphins, a click from one dolphin will be modified in a predictable way as it travels through the fish, especially the swim bladder. In a second mode, a dolphin swimming next to a signaling animal could receive echoes, i.e. information, from the signaler's clicks. This ability has been demonstrated in dolphins (Caldwell \& Caldwell, 1967b; Xitco \& Roitblat, 1996). A third possibility is that dolphins may cue on a click production rate or pattern characteristic of a foraging animal, a strategy used by echolocating bats (Barclay, 1982). In all cases, the non-signaling dolphin obtains information about potential prey using another dolphin's click(s), reducing its own need to echolocate. A prerequisite for the first two mechanisms is that the receiving animal knows the exact structure of the outgoing signal, and while dolphins can alter the source level ( $\mathrm{Au}, 1993$ ) and beam pattern (Amundin, 1991) of their echolocation, the clicks in a given echolocation train vary relatively little ( $\mathrm{Au}, 1993)$. 
The evidence presented in this chapter shows that dolphins foraging in groups echolocate less often than single animals, and some dolphin foraging strategies occur significantly more often in tight groups (e.g. fish whacking and kerplunking, Section 4.3.6). Perhaps these social coordinated foraging strategies are more effective at flushing prey from their refuges, which would reduce the need for echolocation. Kerplunking, for example, occurs when animals are close together and synchronous kerplunks often occur. By working together the dolphins may enjoy the added benefit of not needing to echolocate as much because they can more effectively flush and/or corral prey. These social foraging strategies may, therefore, reduce the reliance on echolocation, and, given the presumed energetic cost of echolocation and the hearing abilities of some fish (Astrup \& Mfhl, 1993; Dunning et al., 1992; Mann et al., 1997), any opportunity to reduce echolocation production is likely to be advantageous.

Single animals also produce whistles in greater numbers when foraging than is expected. This result is especially noteworthy in light of the result that single animals produce fewer whistles than expected when all behaviors are considered (Table 5.2). Whistles have traditionally been considered to function as social signals, but in this case an animal is producing a signal in the absence of other animals so the social function appears less important. In addition, whistles typically lack the energy that would be required to elicit a response from fish (Canfield \& Eaton, 1990). However, whistles, which are relatively low in frequency and non-directional compared to echolocation, can theoretically travel distances that would make them suitable to function as a signal to relatively distant animals, i.e. $>1 \mathrm{~km}$. Two possible functions for such a signal could be a contact call or a food call. Animals $>1 \mathrm{~km}$ from the observation vessel could not be reliably sighted or tracked, but a simple protocol could test this hypothesis. Contact calls and food calls are common in mammalian species (Clark \& Wrangham, 1993; Clark \& Wrangham, 1994; Hauser \& Marler, 1993a; Hauser \& Marler, 1993b), and recent data for bottlenose dolphins show that whistles are recognized by familiar animals (Sayigh, 1992) and are used for cohesion (Janik \& Slater, 1998). The function of a contact call in this circumstance could be to alert conspecifics of a food source, to solicit assistance for some 
type of cooperative foraging, or simply to maintain contact while foraging. To disclose a food source would be logical only if surplus is available, or if an animal could assist kin in finding food. These are testable hypotheses for the Sarasota dolphins as prey fields could be mapped, the relationships of responding animals could be known, and timing of reunions can be monitored.

The findings of the current study indicate that pops differ in function, or at least have a function in addition to the social one reported by Connor and Smolker (1996). The structure of pops recorded in the current study (Figure 5.1c) were similar but not identical to sounds recorded by other researchers ('pops' (Connor \& Smolker, 1996); 'thunks' (McCowan \& Reiss, 1995); and 'low creaks' (dos Santos et al., 1990)). Pops in the current study typically contained energy into higher frequencies than these other sounds, but the durations were comparable. These other studies found social uses for this type of sound. Connor and Smolker (1996), for example, reported that these sounds were used by males to threaten females so the latter would not try to escape from a consortship. The non-social function of the sounds presented here is evidenced by the fact that single animals produced half of the pops recorded in the current study (Table 5.2 ) and that, when produced in multi-animal groups, pops were not observed to mediate any social interactions.

Pops were produced significantly more than expected in one of the main feeding habitats, seagrass edges. Given that acoustic stimuli can trigger a startle response (Blaxter \& Hoss, 1981; Canfield \& Eaton, 1990) and the range of best hearing in dolphin prey is in the relatively low frequencies (Fay, 1988), it is possible that dolphins are using these loud, low frequency rich sounds to flush or startle prey. Prey fish utilize seagrass beds for refuge (Sogard \& Olla, 1993). This environment could prevent their detection due to the visual and acoustic clutter it presents to a searching dolphin. The Sarasota dolphins may use pops simply to startle a fish enough to make it move and/or leave its refuge at which time the dolphin would be better able to detect the fish visually and/or acoustically. This mechanism is similar to that suggested for the kerplunk, and both events either do (pop) or likely (kerplunk) produce intense, low frequency sounds that 
can startle fish (Blaxter \& Hoss, 1981; Canfield \& Eaton, 1990). The hypothesized function of these sounds is different than that suggested by Norris and Møhl (1983) who conjectured that odontocetes can debilitate prey with loud impulsive sounds derived from echolocation. No such debilitation was observed in association with pops or kerplunks, so the proposed role of these events is simply that they elicit a startle response. 


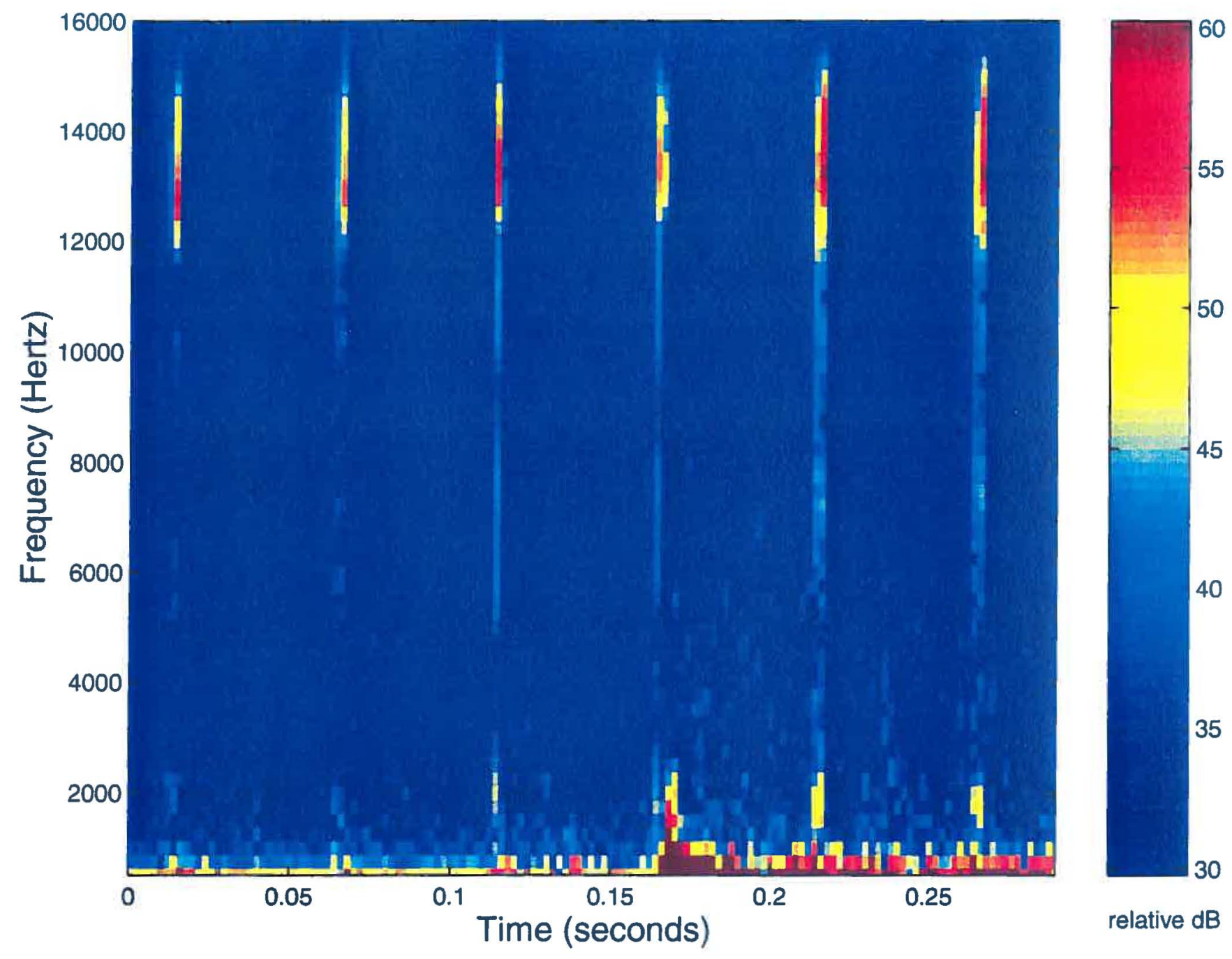

Figure 1a. An example of echolocation clicks recorded by the two-hydrophone towed system. The relative level of the sounds is indicated by the color scale, which maps to the color bar on the right. 


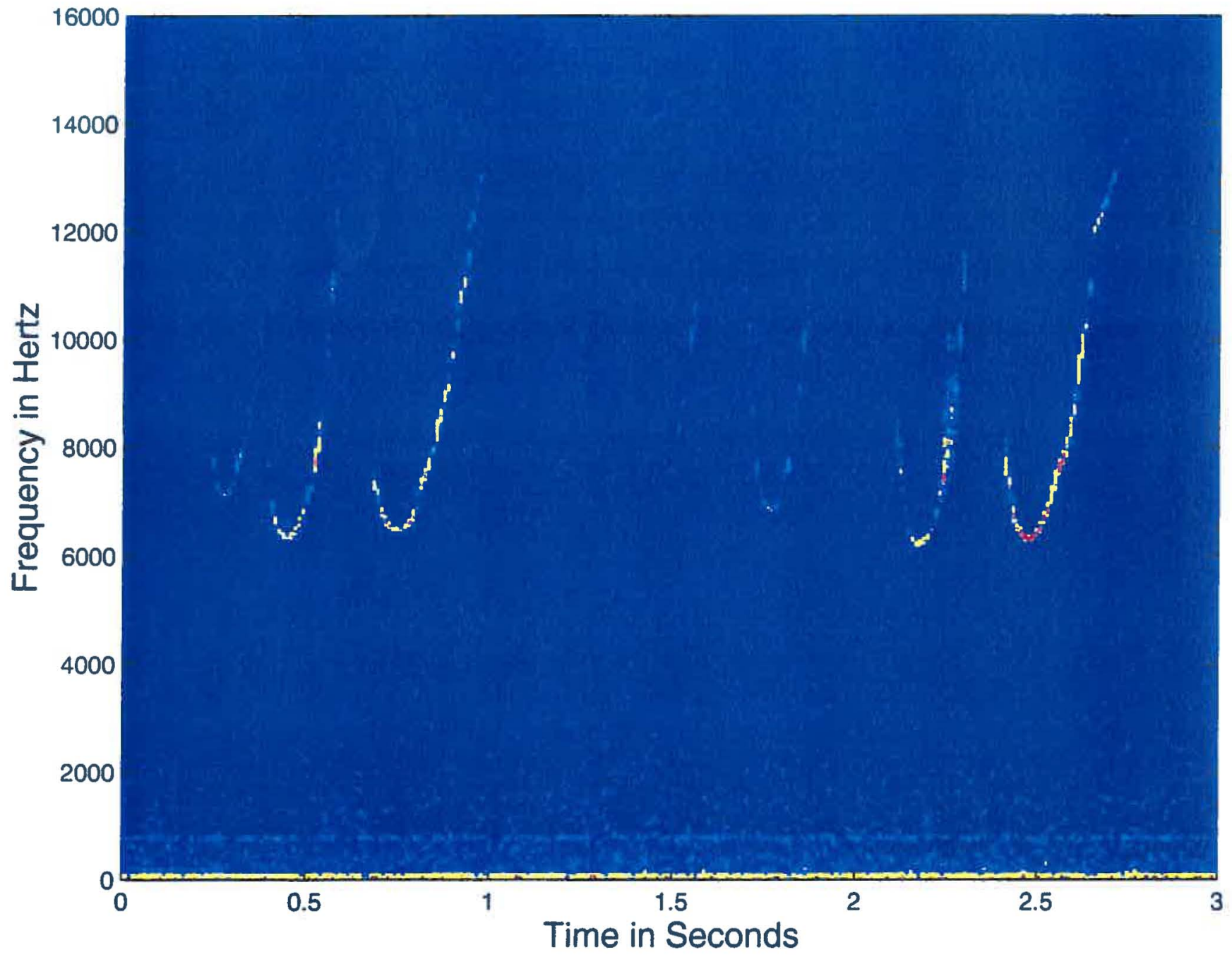

Figure 1b. An example of whistles recorded by the two-hydrophone towed system. The relative level of the sounds is indicated by the color scale, which maps to the color bar on the right. 


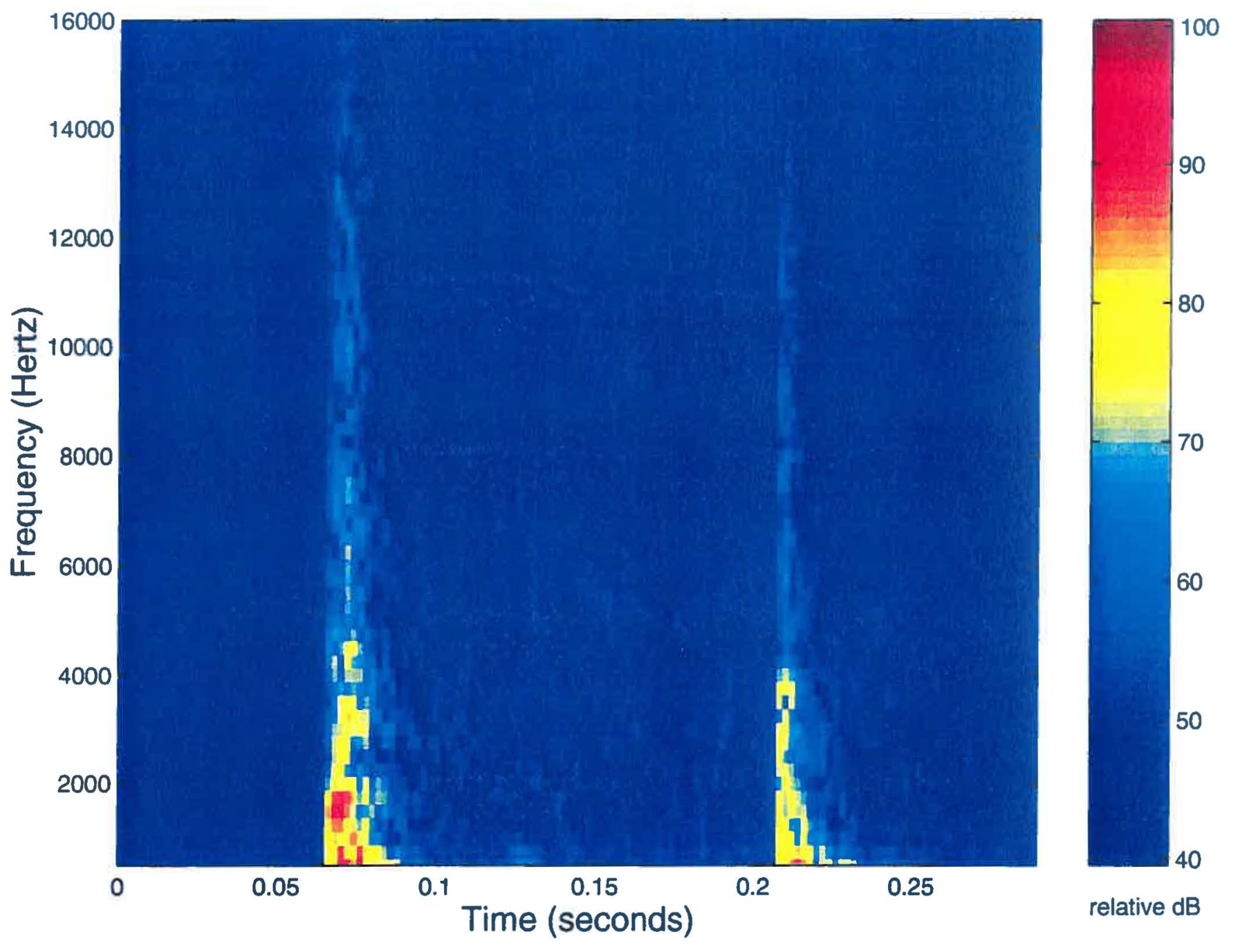

Figure 1c. An example of pops recorded by the two-hydrophone towed system. The relative level of the sounds is indicated by the color scale. which maps to the color bar on the right. 
5.2 a) Overall Rate of Sound Production

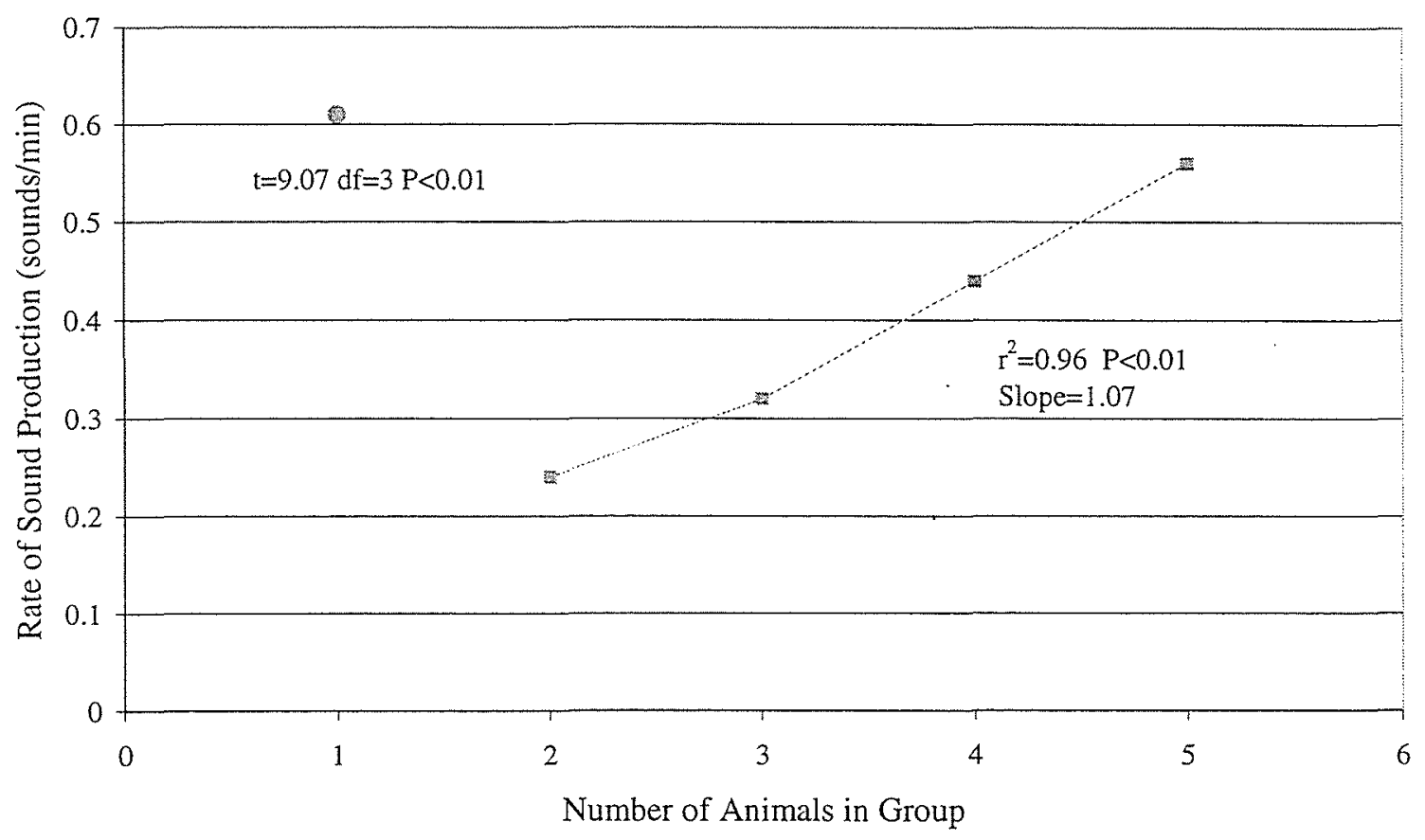


b)

\section{Rate of Sound Production during Foraging}

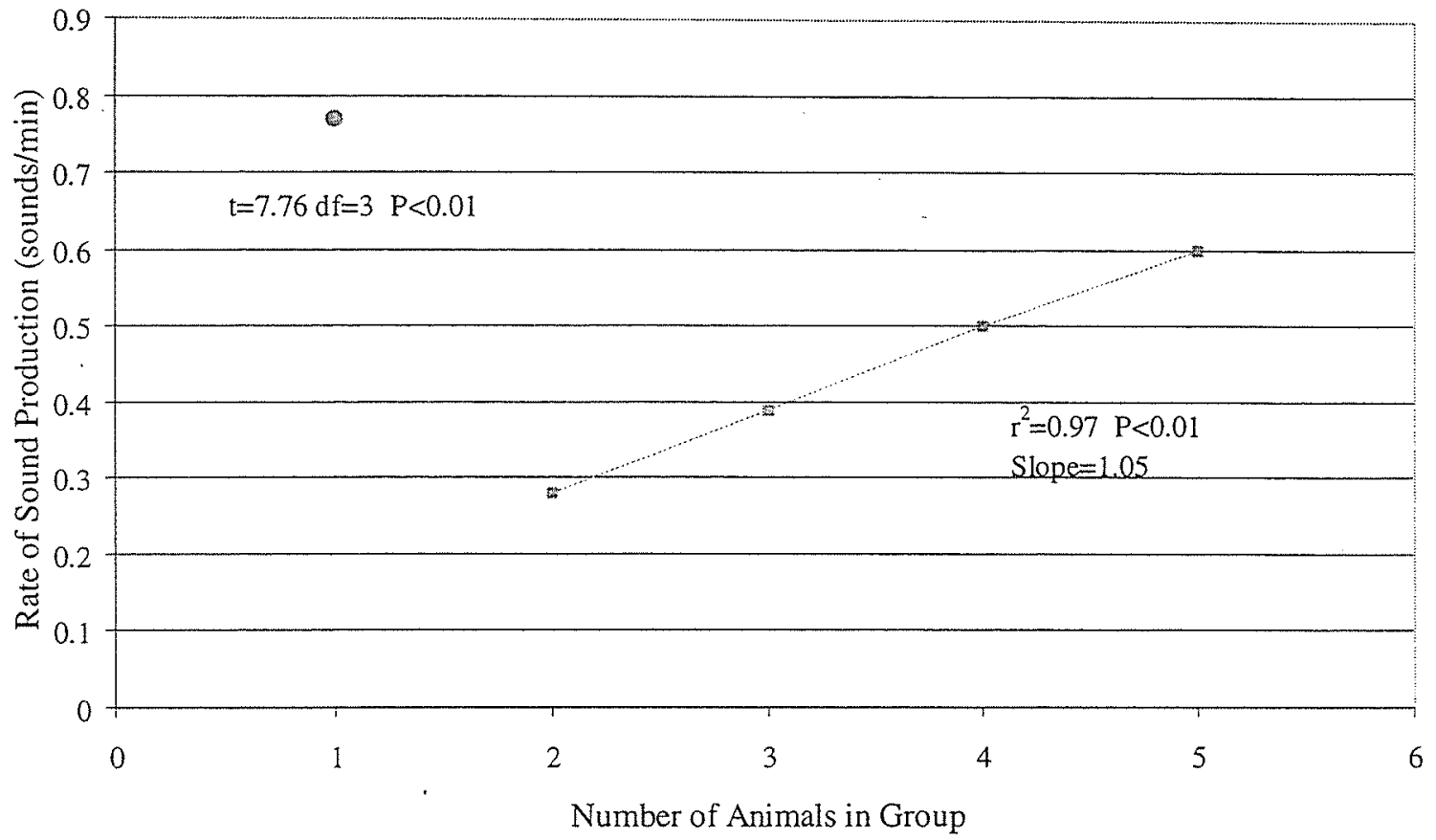

Figures $5.2 \mathrm{a}$ and $\mathrm{b}$. Rate of sound production by group size over all behaviors (a) and during foraging (b). 
Rate of Sound Production - Single vs. Multiple Animal Groups - all Behaviors

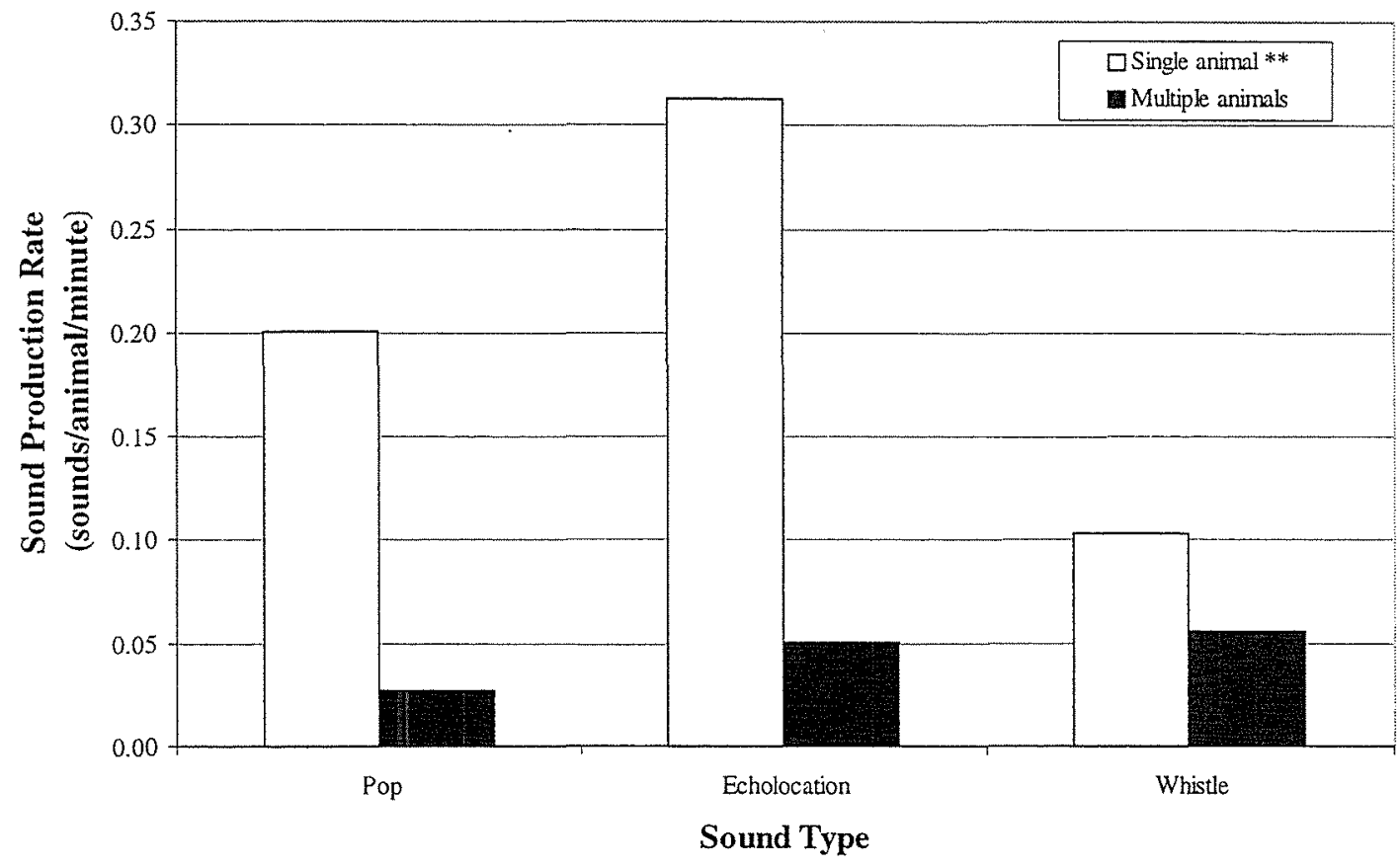

Figure 5.3 Sound production rate for single vs. multiple animal groups across all behaviors $\left(\chi^{2}=39.75, \mathrm{df}=2, \mathrm{P}<<0.01\right)$. 


\section{Rate of Sound Production by Foraging and Non-foraging Groups}

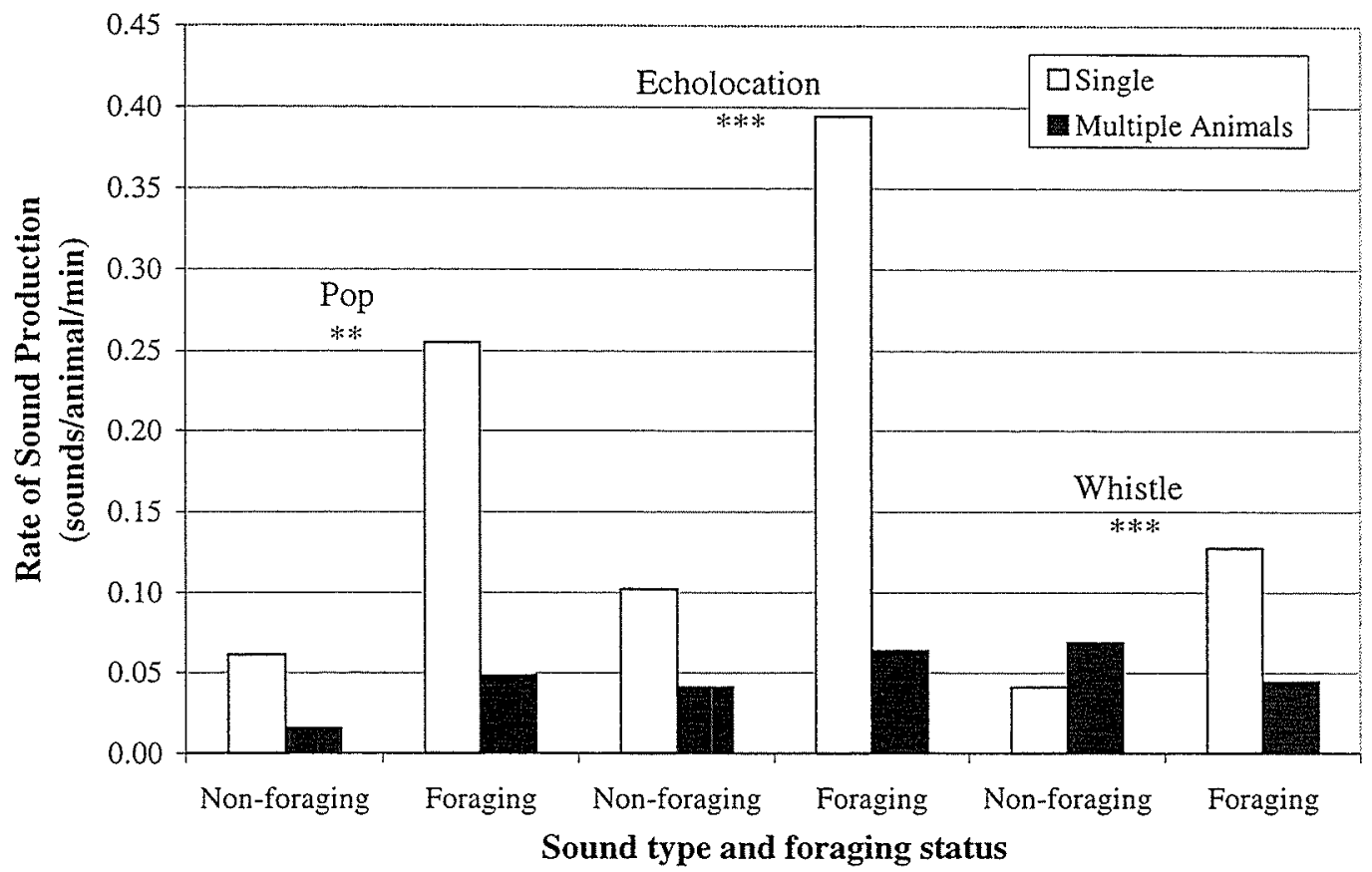

Figure 5.4 Sound production rate and foraging status. Results from condensed contingency table analyses for each sound - group size - foraging status set. See text for $\chi^{2}$ values. $* * P<0.01, * * * P<0.001$ 
Habitat-Specific Rate of Sound Production by Single Foraging Dolphins

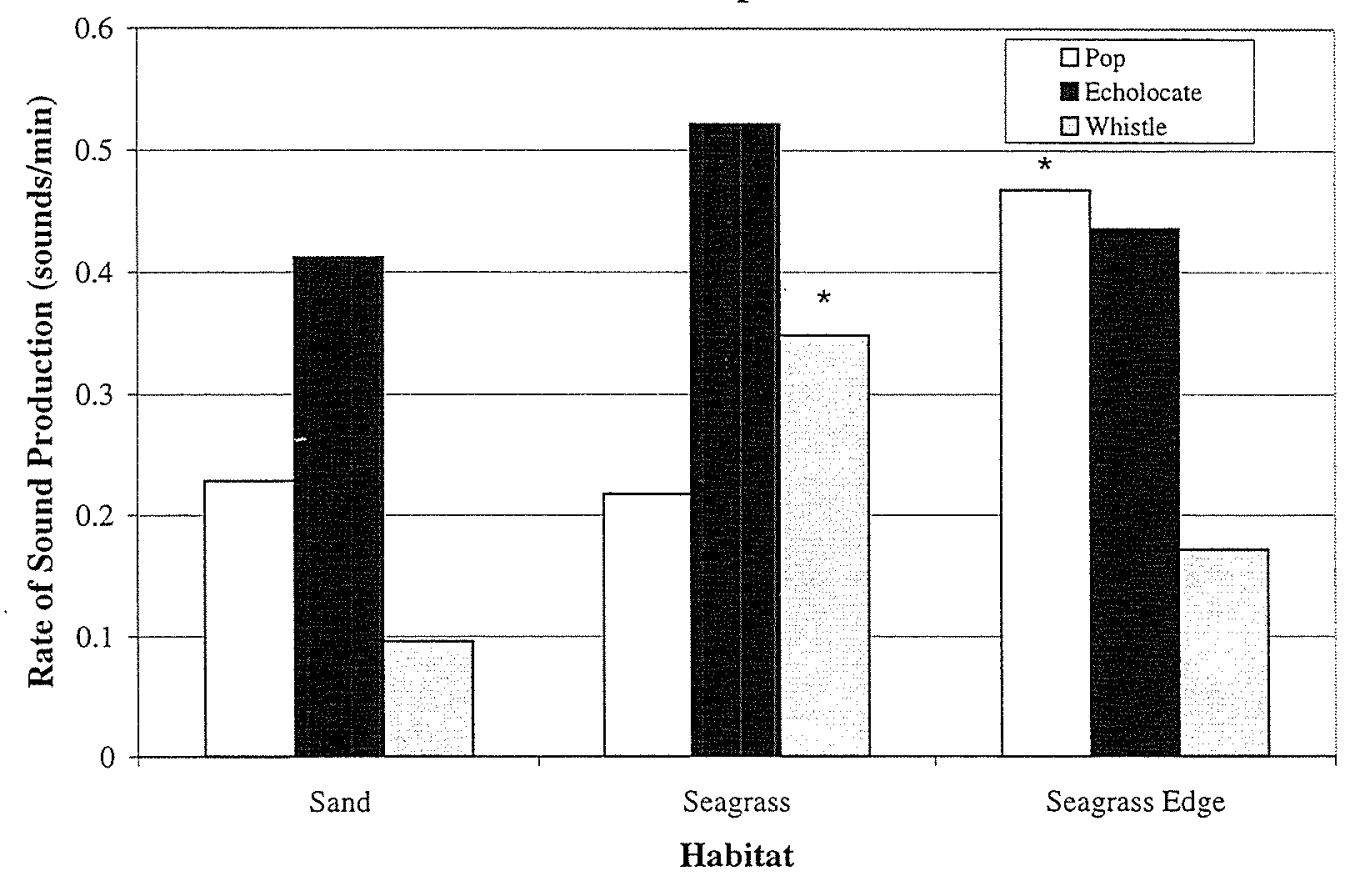

Figure 5.5 Sound production rate for single foraging animals in the three primary habitats. Results from condensed contingency table analyses for each sound-habitat pair. See text for $\chi^{2}$ values. $* \pi \cap n=$ 


\subsection{Literature Cited}

Amundin, M. 1991. Sound production in odontocetes with emphasis on the harbour porpoise, Phocoena phocoena. Stockholm: University of Stockholm.

Astrup, J. and Møhl, B. 1993. Detection of intense ultrasound by the cod (Gadus morhua). Journal of Experimental Biology 182: 71-80.

Au, W. W. L. 1993. The sonar of dolphins, pp. 277. New York: Springer-Verlag.

Barclay, R. M. R. 1982. Interindividual use of echolocation calls: eavesdropping by bats. Behavioral Ecology 10: 271-275.

Barros, N. B. and Wells, R. S. 1998. Prey and feeding patterns of resident bottlenose dolphins (Tursiops truncatus) in Sarasota Bay, Florida. Journal of Mammology 79: 1045-1059.

Blaxter, J. H. S. and Hoss, D. E. 1981. Startle response in herring: the effect of sound stimulus frequency, size of fish, and selective interference with the acousticolateralis system. Journal of the Biological Association of the UK 61: 871-879.

Caldwell, D. K. and Caldwell, M. C. 1967a. Underwater sounds associated with the aggressive behavior in defense of territory by the pinfish, Lagodon rhomboides. Bulletin of the Southern California Academy of Sciences 66: 69-75.

Caldwell, M. C. and Caldwell, D. K. 1965. Individualized whistle contours in bottlenosed dolphins (Tursiops truncatus). Nature 207: 434-435.

Caldwell, M. C. and Caldwell, D. K. 1967b. Intraspecific transfer of information via the pulsed sound in captive odontocete cetaceans. In Animal Sonar Systems, vol. 2 (ed. R.-G. Busnel), pp. 879-936. Jouy-en-Josas: NATO Advanced Study Institute.

Caldwell, M. C., Haughen, R. M. and Caldwell, D. K. 1962. High-energy sounds associated with fright in the dolphin. Science 138: 907-908.

Canfield, J. G. and Eaton, R. C. 1990. Swimbladder acoustic pressure transduction initiates Mauthner-mediated escape. Nature 347: 760-762.

Clark, A. P. and Wrangham, R. W. 1993. Acoustic analysis of wild chimpanzee pant hoots: Do kibale forest chimpanzees have an acoustically distinct food arrival pant hoot? American Journal of Primatology 31: 99-109.

Clark, A. P. and Wrangham, R. W. 1994. Chimpanzee arrival pant-hoots: Do they signify food or status? International Journal of Primatology 15: 185-205. 
Connor, R. C. and Smolker, R. A. 1996. 'Pop' goes the dolphin: a vocalization male botttlenose dolphins produce during courtships. Behaviour 133: 643-662.

dos Santos, M. E., Caporin, G., Moreira, H. O., Ferreira, A. J. and Coelho, J. L. B. 1990. Acoustic behavior in a local population of bottlenose dolphins. In Sensory Abilities of Cetaceans (ed. J. Thomas and R. Kastelein), pp. 585-598. New York: Plenum Press.

Dunning, D. J., Ross, Q. E., Geoghegan, P., Reichle, J. J., Menezes, J. K. and Watson, J. K. 1992. Alewives avoid high-frequency sound. North American Journal of Fisheries Management 12: 407-416.

Everitt, B. S. 1977. The Analysis of Contingency Tables. In Mongraphs on Applied Probability and Statistics (ed. M. S. Bartlett and D. R. Cox), pp. 128. London: Chapman and Hall.

Fay, R. R. 1988. Hearing in Vertebrates: a Psychophysics Databook, pp. 621. Winnetka, IL: Hill-Fay.

Fish, M. P. and Mowbray, W. H. 1970. Sounds of western north Atlantic fishes: a reference file of biological underwater sounds, pp. 207. Baltimore: Johns Hopkins Press.

Griffin, D. R. 1958. Listening in the Dark, pp. 413. New Haven: Yale University Press.

Hauser, M. D. and Marler, P. 1993a. Food-associated calls in rhesus macaques (Macaca mulatta): I. socioecological factors. Behavioral Ecology 4: 194-205.

Hauser, M. D. and Marler, P. 1993b. Food-associated calls in rhesus macaques (Macaca mulatta): II. costs and benefits of call production and suppression. Behavioral Ecology 4: 206-212.

Holler, P. 1995. Orientation by the bat Phyllostomus discolor (Phyllostomidae) on the return flight to its resting place. Ethology 100: 72-83.

Janik, V. M. 1997. Food calling in bottlenose dolphins (Tursiops truncatus). Advances in Ethology 32: 123.

Janik, V. M. and Slater, P. J. B. 1998. Context-specific use suggests that bottlenose dolphin signature whistles are cohesion calls. Animal Behaviour 56: 829-838.

Mann, D. A., Lu, Z. and Popper, A. N. 1997. A clupeid fish can detect ultrasound. Nature 389: 341. 
McCowan, B. and Reiss, D. 1995. Maternal aggressive contact vocalizations in captive bottlenose dolphins (Tursiops truncatus): wide-band, low-frequency signals during mother/aunt-infant interactions. Zoo Biology 14: 293-309.

Miller, L. A. 1983. How insects detect and avoid bats. In Neuroethology and Behavioral Physiology (ed. F. Huber and H. Markl), pp. 251-266. Berlin, Heidelberg: Springer-Verlag.

Neuweiler, G. and Mohres, F. P. 1966. The role of spatial memory in the orientation of bats. In Les Systemes Sonars Animaux Biologie et Bionique (ed. R. G. Busnel), pp. 129-140. Frascati: Nato Advanced Study Institute.

Norris, K. S. and Mohl, B. 1983. Can odontocetes debilitate prey with sound? The American Naturalist 122: 85-104.

Popper, A. N. 1980. Sound emission and detection by delphinids. In Cetacean behavior (ed. L. M. Herman), pp. 1-53. New York: John Wịley and Sons.

Roeder, K. D. 1962. The behaviour of free flying moths in the presence of artificial ultrasonic pulses. Animal Behavior 10: 300-304.

Roeder, K. D. 1967. Turning tendency of moths exposed to ultrasound while in stationary flight. Journal of Insect Physiology 13: 873-888.

Roeder, K. D. and Treat, A. E. 1957. Ultrasonic reception by the tympanic organ of noctuid moths. Journal of Experimental Zoology 134: 127-158.

Roeder, K. D., Treat, A. E. and Vande Berg, J. S. 1968. Auditory sense in certain sphingid moths. Science 159: 331-333.

Ryan, M. J. and Tuttle, M. D. 1983. The ability of the frog-eating bat to discriminate among novel and potentially poisonous frog species using acoustic cues. Animal Behavior 31: 827-833.

Ryan, M. J., Tuttle, M. D. and Taft, L. K. 1981. The costs and benefits of frog chorusing behavior. Behavioral Ecology and Sociobiology 8: 273-278.

Sayigh, L. S. 1992. Development and functions of signature whistles of free-ranging bottlenose dolphins, Tursiops truncatus. In Biology, pp. 344. Woods Hole, MA: Woods Hole Oceanographic Institution and Massachusetts Institute of Technology.

Sayigh, L. S., Tyack, P. L. and Wells, R. S. 1993. Recording underwater sounds of freeranging dolphins while underway in a small boat. Marine Mammal Science 9: 209-213. 
Sayigh, L. S., Tyack, P. L., Wells, R. S. and Scott, M. D. 1990. Signature whistles of free-ranging bottlenose dolphins, Tursiops truncatus: stability and motheroffspring comparisons. Behavioral Ecology and Sociobiology 26: 247-260.

Sayigh, L. S., Tyack, P. L., Wells, R. S., Scott, M. D. and Irvine, A. B. 1995. Sex differences in signature whistle production of free-ranging bottlenose dolphins, Tursiops truncatus. Behavioral Ecology and Sociobiology 36: 171-177.

Simmons, J. A., Fenton, M. B. and O'Farrell, M. J. 1979. Echolocation and pursuit of prey by bats. Science 203: 16-21.

Sogard, S. M. and Olla, B. L. 1993. The influence of predator presence on utilization of artificial seagrass habitats by juvenile walleye pollock, Theragra chalcogramma. Environmental Biology of Fishes 37: 57-65.

Tuttle, M. D. and Ryan, M. J. 1981. Bat predation and the evolution of frog vocalizations in the neotropics. Science 214: 677-678.

Tyack, P. L. 1997. Studying how cetaceans use sound to explore their environment. In Perspectives in Ethology, vol. 12 (ed. D. H. Owings, M. D. Beecher and N. S. Thompson), pp. 251-297. New York: Plenum Press.

Whitehead, H. and Weilgart, L. 1990. Click rates from sperm whales. Journal of the Acoustical Society of America 87: 1798-1806.

Wood, F. G. J. 1953. Underwater sound production and concurrent behavior of captive porpoises, Tursiops truncatus and Stenella plagiodon. Bullentin of Marine Science for Gulf and Caribbean 3: 120-133.

Xitco, M. J. and Roitblat, H. L. 1996. Object recognition through eavesdropping: Passive echolocation in bottlenose dolphins. Animal Learning and Behavior 24: 355-365. 


\section{Chapter 6. Conclusions}

Previous qualitative studies of bottlenose dolphin foraging (Bel'kovich et al., 1991; Shane, 1990) provided some observational background (e.g. descriptions of some feeding behaviors) and theoretical basis for the current, quantitative research into the sequence and ecology of specific foraging behaviors displayed by Sarasota, FL bottlenose dolphins. New observational techniques developed as part of the current study allowed a more detailed view of foraging behavior including the discovery of new foraging behaviors for the Sarasota dolphins. The new behavioral observation tool, an overhead video system, confirmed the fact that surface behavior, especially with regard to foraging behavior in this work, is not a representative sample of behavior. Many behaviors observed to occur below the surface, for example, were never seen at the surface, and some of those seen at the surface occurred more frequently below than at the surface. This ability to see through or at least well into the water column has also definitively linked suspected or probable foraging behaviors with confirmed feeding events. Careful, quantitative sampling of specified behaviors documented significant links between them and prey capture. In addition, this detailed sampling revealed several distinct foraging strategies.

Behavioral sequences analyzed in reverse chronological order beginning with a successful capture event and retreating one behavior at a time (i.e. a state-lag analysis) demonstrated that dolphins do organize their behaviors into specific sequences (Figures 4.7-12). The sequences are not deterministic, showing variability based on factors including, but not limited to, habitat, individual preference, and nearest neighbor distance. While the sequences were not deterministic several specific patterns predominated. These patterns defined three distinct foraging strategies: 'rooting', 'kerplunking', and 'fish whacking'. Rooting and kerplunking are two search strategies that appear to focus on the need to flush or startle prey hidden in refuges so as to make them more detectable. Alternatively, these two behaviors may act to corral prey within those refuges therefore increasing the prey density in the refuge before venturing in to 
actually find the prey. Rooting, a behavior in which a dolphin explores the bottom or some object by orienting itself almost vertically and stationary in the water column, had not been reported previously in Sarasota but has been documented for dolphins in other areas (Rossbach \& Herzing, 1997). Kerplunking has been observed in Sarasota as well as in other areas ( $\mathrm{R}$. Connor, pers comm). Kerplunking consists of a dolphin near the surface raising its tail flukes out of the water and then forcefully bringing them down to the surface and continuing this forceful movement well into the water column. These two strategies share a similar pursuit sequence consisting of behaviors such as 'accelerate' and 'side-swimming'. Leatherwood (1975) described side-swimming as well as the rapid direction change maneuver called a 'pinwheel' which is preceded sideswimming in Sarasota dolphins. The third foraging strategy, fish whacking, had also been previously described for Sarasota dolphins (Wells, Scott \& Irvine, 1987), but its dependence on factors such as habitat and nearest neighbor distance and its unique overall sequence were not previously documented. Fish whacking is also preceded by side-swimming, but its relatedness to the other two strategies ends there. This study has elucidated the ecology and function of these and other behaviors (e.g. 'scan' which had only been observed in captive dolphins) described in previous qualitative studies of feeding dolphins.

The use of these three strategies varies at least by habitat and individual. Rooting occurred more often in sandy bottom habitats, kerplunking at the edges of seagrass meadows, and fish whacking within the seagrass meadows. In addition to this habitat specificity two of the three strategies (kerplunking and fish whacking) were social as they occurred only when animals were in close proximity and involved highly synchronous behavior. The usage of these behaviors suggests that dolphins must invest in not only learning specific strategies and selecting the appropriate habitat, but also in coordinating with another individual. To justify this investment, use of these strategies must result in significant foraging as well as perhaps social benefits. Whether the observed behaviors represent the complete foraging repertoires for any or all individuals in this study is unknown, and other foraging behaviors are observed in Sarasota. Therefore it is also not known whether the observed sequences are determined by an individual's foraging 
behavior repertoire, the habitat, or other factors such as the behavior of the prey. All individuals, for example, may use the same strategy in a particular habitat, or individuals may seek out the appropriate habitat for the strategies in their repertoire. Whatever the case, the current study demonstrated that distinct foraging strategies do exist, and presumably a certain amount of time is required for an individual to acquire a particular behavior. If this cost were high then the presence of many strategies in the repertoire of a single individual would not be expected. If, however, behaviors were readily transmitted from parents or other close associates to young individuals, for which anecdotal evidence was presented in this work, then all strategies to which an individual is exposed would be expected to appear in its repertoire. Further study could answer these questions with detailed longitudinal observations beginning at birth of particular individuals.

Acoustic data collected by the overhead video/underwater audio observation platform revealed that echolocation is associated with foraging. Sound use patterns were significantly affected by whether or not an animal was foraging and whether it was alone or part of a larger group. While the overall rate of sound production was low, single animals (i.e. no other animals visible from observation vessel) had significantly higher rates of producing all three sounds recorded: clicks, whistles, and pops. This difference occurred only during foraging as the single, non-foraging animal sound production rates were consistent with the per-animal rate in multiple-animal groups. The use of sounds, therefore, is crucially important to single foraging animals. This reliance on acoustics is somewhat mitigated for animals foraging in groups larger than one. For echolocation and pops both single and multi-animal groups increased their use during foraging, but for whistles single animals increased their use while animals in larger groups decreased their production.

The production of pops and whistles by single foraging animals was influenced by the habitat in which the animal was foraging. Pops were significantly more common at seagrass edges, and significantly more whistles than expected were recorded as single animals foraged within seagrass meadows. Echolocation production did not significantly deviate from expected values across the three major habitats. 
Echolocation and whistle rates of free-ranging dolphins were also recorded using an attached acoustic data logger, and the data revealed similarly low overall rates of sound production. This data logger (Nowacek et al., 1998) provided acoustic records known unambiguously to have been produced by a single individual. Based on an experimentally determined sensor location, every click or whistle event was recorded by the device. Relatively long periods of silence (tens of minutes) were punctuated by short bursts of sound production. The predominant behavior observed during these experiments was traveling. These results indicate that dolphins do not need to continuously click to navigate. Likewise, whistles are not produced at very high rates even by mothers traveling with dependent calves, a result consistent with Sayigh (1992).

Dolphin foraging behavior had previously been described through qualitative, anecdotal reports primarily of groups of animals. This thesis, using continuous observations of individual animals, has demonstrated a discrete repertoire of foraging behaviors used by Sarasota bottlenose dolphins. While this list of behaviors is certainly not exhaustive, it is representative both of the repertoire and the ecology of foraging behaviors. The occurrence of a given behavior depends on which behaviors have occurred previously, the habitat in which the dolphin is foraging, and the individual performing the behaviors. Other factors not measured but that certainly would affect the use of a particular behavior include the type, behavior, and distribution of prey. In addition, the observed foraging behaviors appear to focus on the need to flush and/or corral prey hiding in refuges. The use of sound is also important for foraging Sarasota dolphins. Production of all three sound types increases dramatically in single animals when foraging, and echolocation and pop production increase in groups in which at least the focal individual was foraging. Habitat also influences the use of some sounds in single foraging animals. Whistles are more common than expected in seagrass meadows and pops at the seagrass edges, but echolocation is common is all habitats perhaps indicating its more central role in the foraging process. 


\section{Literature Cited}

Bel'kovich, V. M., Ivanova, E. E., Yefremenkova, O. V., Kozarovitsky, L. B. \& Kharitonov, S. P. (1991). Searching and hunting behavior in the bottlenose dolphin (Tursiops truncatus) in the Black Sea. In Dolphin Societies: Discoveries and Puzzles (ed. K. Pryor and K. S. Norris), pp. 38-67. University of California Press, Berkeley, CA.

Leatherwood, S. (1975). Some observations of feeding behavior of bottle-nosed dolphins (Tursiops truncatus) in the northern Gulf of Mexico and (Tursiops cf $T$. gilli) off southern California, Baja California, and Nayarit, Mexico. Marine Fisheries Review 37: 10-16.

Nowacek, D. P., Tyack, P. L., Wells, R. S. \& Johnson, M. P. (1998). An onboard acoustic data logger to record biosonar of free-ranging bottlenose dolphins. Journal of the Acoustical Society of America 103: 1409-1410.

Rossbach, K. A. \& Herzing, D. L. (1997). Underwater observations of benthic-feeding bottlenose dolphins (Tursiops truncatus) near Grand Bahama Island, Bahamas. Marine Mammal Science 13: 498-504.

Sayigh, L. S. (1992). Development and functions of signature whistles of free-ranging bottlenose dolphins, Tursiops truncatus. Ph.D. dissertation thesis, Woods Hole Oceanographic Institution and Massachusetts Institute of Technology.

Shane, S. H. (1990). Behavior and ecology of the bottlenose dolphin at Sanibel Island, Florida. In The Bottlenose Dolphin (ed. S. Leatherwood and R. R. Reeves), pp. 245-265. Academic Press, San Diego.

Wells, R. S., Scott, M. D. \& Irvine, A. B. (1987). The social structure of free-ranging bottlenose dolphins. In Current Mammalogy, vol. 1 (ed. H. H. Genoways), pp. 247-305. Plenum Press, New York. 


\section{Appendix 1. Overhead Video/Underwater Acoustic Observation Platform}

\section{AI.I Introduction}

A systematic and consistently applied sampling regimen is necessary to obtain unbiased data for the study of animal behavior. Numerous sampling methods are available to the behavioral researcher each with strengths, weaknesses, and particular applications. The following discussion is based on the methods explained by Altmann (1974). To obtain quantitative, detailed behavioral data the method of choice for many research questions is to apply continuous and point sampling protocols during focalindividual follows. Continuous data can be used to establish rates and frequencies of behaviors, evaluate behavior sequences, and are the richest source of data on social behavior and relationships. Carefully designed point sampling is useful for determining activity budgets or diurnal behavior patterns. For continuous sampling the researcher must be able to observe an identifiable subject for long periods of time. Visual contact may not be continuous throughout a sampling interval, but if the 'time-out' periods are recorded one can still precisely measure durations, rates, and frequencies of behaviors and behavioral transitions. For marine mammal research, however, the 'time-outs' are usually longer than the 'time-ins', and the 'time-ins' occur almost exclusively at the surface which is not a representative sample of behavior (see Section 4.3.5). This observation presents a problem for Mann's (1999). assertion that, for short 'time-outs', an animal's behavior when in view (e.g. at successive surfacings) can be extrapolated to when not in view. For other sampling methods, e.g. point or scan sampling, the subject's behavior is recorded at predetermined moments in time (Altmann, 1974). The times at which marine mammal subjects, however, are visible are often determined by the animal not the observer. Therefore, for both continuous and point sampling, unbiased quantitative samples are difficult to obtain. 
Studying animals that inhabit clear water environments provides a simple solution for marine mammal researchers, and at least three observational techniques have been utilized to exploit these conducive environments. First, boat-based observers can maintain visual contact of individuals for relatively long periods, but their presence may influence the animals' behavior (Table 1). Smolker (1993) for example, followed focal individuals for periods of up to 9 hours with a median observation session lasting 3.5 hours. A large quantity of continuous data are easily obtained in such circumstances, although some of the observation time is lost as the animals may submerge out of sight even in these more favorable habitats (Connor \& Smolker, 1996). Also, if reflection is negligible then continuous video recording of an animal's behavior is also possible ( $R$. Connor, pers. comm.) which can be invaluable if the behaviors being sampled occur in rapid succession or are of short duration. Observing animals from an underwater platform or while diving is another means of exploiting clear water environments. In the Bahamas, for example, continuous sampling for periods of 5-35 minutes can be conducted while diving (Dudzinski, Clark \& Würsig, 1995; Herzing, 1996). Caveats associated with this type of observation include the potential biases introduced by having a human diver near the animals and that the subject controls the duration of the observation period. Norris (1991) reviews the use mobile underwater viewing platforms, a tool that has been effectively utilized (Norris et al., 1994; Ostman, 1994; Pryor \& Norris, 1991). Such an observation platform allows the observer to maintain visual contact with swimming animals and observe for periods of tens of minutes, but it can be used only in the calmest sea states (Ostman, 1994). Studies conducted in clear water environments provide excellent data on the behavior of individuals in these populations (Connor \& Smolker, 1996; Ostman, 1994; Smolker et al., 1993) but do have limitations (see Table 1).

Environments in which the water is more opaque provide limited visibility of submerged animals. Part of the inability to see into the water column in these environments is caused by reflection and refraction of sunlight. Viewing from overhead 
mitigates these lighting problems and can extend continuous observation of marine mammals to more opaque waters. For an observer to see into the water, light must penetrate the water. Reflection is at a minimum at the zenith angle, i.e. $0^{0}$ relative to incident light, and increases only slightly to $50^{\circ}$ where it begins to increase dramatically (Williams, 1970). Therefore, if one can observe from an angle close to that of incident light, reflection is minimized and illumination of the water is maximized. In regards to refraction, Snell's law states that the image the eye sees is distorted by an amount proportional to the sine of the angle of incident light. The amount of distortion is minimized, therefore, as the observation point approaches the angle of incident light.

Observing from near shore cliffs is one method that takes advantage of an elevated vantage point. Cliff-based observations have unique advantages and disadvantages (Table 1). Some researchers have conducted studies of cetacean behavior and activity budgets from cliffs (Hanson \& Defran, 1993; Jefferson, 1987; Norris et al., 1994; Osborne, 1986), but even from this elevated observation point their samples are mostly limited to surface behavior. In addition, repeatedly identifying individuals from such a distance is virtually impossible for small cetaceans, so sampling is limited to scan sampling of focal groups (Altmann, 1974). For behavioral studies of large whales observations from cliffs can be more effective as individuals can be repeatedly identified and thereby tracked for long periods (Richardson et al., 1995). In such circumstances the use of point sampling methods and possibly continuous sampling may be possible, although detailed behavior is rarely visible from such a vantage point. A cliff-based observational technique that does not rely on seeing submerged animals is tracking animal movements using a surveyor's theodolite to precisely locate each successive surfacing (Table 1). Theodolite data have been utilized, for example, to measure animals' responses to acoustic playbacks (Malme et al., 1983), swim speeds, distance from shore, and distances traveled (Jefferson, 1987; Norris et al., 1994; Richardson et al., 1995; Sumich, 1983). While observing from cliffs permits theodolite tracking and the 
collection of some behavioral data, observations are normally limited to coarsely defined behaviors of specific habitats and subject species.

A mobile, overhead observation platform provides an excellent means to sample populations that do not inhabit clear water or areas near an elevated shoreline. Aerial surveys from airplanes have been effectively utilized for many years to assess population size and distribution of various species of mysticetes (Hain et al., 1992; Hubbs \& Hubbs, 1967; Nerini \& Rugh, 1986), but caution must be exercised as environmental factors can introduce biases (Nerini \& Rugh, 1986). For behavioral study, the most extensive use of aerial observations has been in the study of bowhead whale (Balaena mysticetus) behavior and reaction to human activity (Richardson et al., 1995; Richardson et al., 1985; Würsig et al., 1985; Würsig et al., 1989). The aerial observations were conducted according to a protocol which minimized disturbance to the whales (see (Richardson $e t$ al., 1985; Richardson \& Malme, 1993). Although the aerial observations did provide an undisturbed sample, they provided only few detailed behavioral data of submerged animals (Würsig et al., 1985). This restricted underwater viewing was likely due to the turbid water and the constraints imposed by the non-disturbance protocol, specifically, the need to maintain an altitude of $450-600 \mathrm{~m}$ and to circle at a lateral range of at least 0.5 $\mathrm{km}$ which compromises the benefits of viewing from overhead by increasing the zenith angle (Richardson et al., 1985; Richardson \& Malme, 1993). While some types of behavioral data for these whales are made more accessible by observations from airplanes, e.g. near surface feeding (Watkins \& Schevill, 1979), this accessibility is in part due to the large size and relatively slow swim speeds of the bowhead which would also be true of other slow-moving, solitary baleen whales as well as the larger odontocetes. Aerial photographs from helicopters have been used to assess stock size and structure of smaller, faster moving odontocetes (Perryman \& Lynn, 1994; Scott, Perryman \& Clark, 1985). These species, however, would not be good subjects for behavioral study using these methods because individual animals would be difficult to 
follow, especially from altitudes such as those suggested by Richardson (1985) to minimize disturbance for baleen whales.

Given the restrictions of helicopters and airplanes, a need exists for a mobile observation platform that can take advantage of the benefits of an overhead vantage point while being relatively unobtrusive to the animals under observation.

\section{A1.2 Mobile Overhead Video Observation Platform}

Considering the demands of systematic behavioral sampling, the difficulty of meeting those demands in most environments with boat-based observations, and the optical benefits of viewing from overhead we recognized a niche for a mobile, relatively unobtrusive overhead video platform from which we could observe and record the behavior of marine mammals. The system permits continuous viewing of a focal individual in environments where such sampling is otherwise difficult to impossible. In addition, the system can provide video footage subject to less reflection and refraction for both these environments and those in which continuous observations are already possible. To be an effective tool for continuous focal-animal sampling we designed this system such that it could maintain an animal within the field of view for tens of minutes and provide images of sufficient resolution to observe detailed behaviors.

\section{A1.2.1 System Configuration and Operation}

The system consists of seven major components: 1) A helium filled aerostat which is tethered to the boat; 2) Remote controlled video camera suspended from the aerostat; 3) Combined electrical/strength tether that brings video images down and carries power and control signals from the support vessel; 4) Video recorder and monitor; 5) Camera base unit that is the interface for camera control and power; 6) two towed hydrophones, the signals from which are recorded directly onto the video tape with the option of being filtered before recording; and 7) an appropriate support vessel providing protection for equipment, adequate operating space, as well as a padded cradle for safe 
docking of the aerostat. An operator watching the video monitor controls the pan-tilt, zoom, focus, and iris from the camera base unit. This real-time image feedback and adjustment capability is crucial to the efficacy of the system. The video signal feeds directly from the camera into the recorder to minimize generational loss, then to the monitor. Figure 1 shows the entire system.

Current components of the system include a $1500 \mathrm{ft}^{3}$ aerostat (Aerostar International TRF1500), custom camera and pan-tilt system (Industrial Video Systems), combined electrical and mechanical tether (Cortland Cable), digital video recorder (Sony HR1000), and Sony Trinitron monitor. The aerostat carries a small aluminum bracket to which the camera is bolted; this relatively rigid attachment minimizes wind induced camera vibration. Also adding to the stability of the platform is the rigid-fin aerostat. Previously an inflatable-fin model was used, but this airship was unstable in the presence of relatively small airflow disturbances. For the camera unit we specified features based on the need to obtain clear images of fast-moving animals. The camera is a 3-chip color (JVC model KY-F55BU) providing 750 lines of horizontal resolution. All camera features can be controlled remotely: the lens aperture (iris); interchangeable zoom lenses (e.g. $8-80 \mathrm{~mm}$ or $12-120 \mathrm{~mm})$; pan $\left(360^{\circ}\right.$ continuous at up to $100^{\circ}$ per second); and tilt $\left(90^{\circ}\right)$. The pan and tilt are operated with a joystick and are not mutually exclusive control signals. The combined electrical/mechanical tether was also a revision from the earlier system. A neoprene jacketed kevlar shell (1200 lb. breaking strength) provides protection for the fiber optic line which carries control signals to the camera and video from the camera, power cables, and a separate RG-179 coaxial cable available for an additional fixed camera, e.g. wide angle. All of these features contribute to a system that can address specific behavioral research questions and operate in a range of field conditions.

This system has been operated from a relatively small boats $(<6 \mathrm{~m})$ and is intended for use in relatively calm inshore or near-shore waters. Suitable weather 
conditions for operating the system include winds $\leq 15$ knots and no rain. A darkened enclosed monitoring area to reduce glare on the video monitor has proven integral to successful operation. Glare off the water surface often associated with a sunspot can impede observations, but careful positioning of the boat relative to the subjects minimizes this problem. Polarizing filters do not improve the image and often result in dark images because advantageous as well as interfering polarizations of light are filtered out as the lens spins with focus and zoom. In regards to any disturbance introduced by the system, the only behavioral reaction noted has been in response to the shadow of the aerostat. Approximately once per six hours of observation a brief $(<10 \mathrm{sec})$ cessation of activity occurred as the shadow passed over an animal, but the animal invariably returned to its pre-disturbance activity.

\section{A1.2.2 Applications of the System}

To date the system has been used in eight studies of three different marine mammal species. Video footage has been used to 1) estimate the life-stage of manatees (Trichechus manatus) along the west coast of Florida (Flamm et al., 1999. In Prep); 2) to study the foraging behavior of bottlenose dolphins (Tursiops truncatus) (Nowacek et al., 1995); 3) to document the subsurface responses of bottlenose dolphins to vessel approaches (Nowacek, 1999); 4) to collect pilot data on movement patterns and behavior of Puget Sound killer whales (Nowacek et al., 1995); 5) to record interactions between human swimmers and manatees; 6) to investigate the disturbance of manatees by boats; 7) to record the behavior and acoustic activity of male bottlenose dolphins; and 8) to measure the swimming speed of wild bottlenose dolphins (Rohr et al., 1998). In a detailed example of the system's application, the detailed sequences of behaviors leading to prey capture have been recorded for bottlenose dolphins (e.g. head scanning, rooting, see Section 4.3.2). In addition to these applications the overhead video system could be an effective tool to study the spatial composition or relationships of groups of animals. Also, in combination with a sound localization system (e.g. hydrophone array (Miller \& 
Tyack, 1998; Spikes \& Clark, 1996), the overhead video could be used to corroborate the locations of vocalizing animals while recording the behavior of that individual as well as other nearby animals. The system could also be used in a 'stationary' mode to monitor a particular area of interest, e.g. critical foraging habitat or important travel corridors/passes. 


\begin{tabular}{|c|c|c|c|}
\hline $\begin{array}{c}\text { Observation } \\
\text { Technique }\end{array}$ & Advantages & Disadvantages & References \\
\hline $\begin{array}{l}\text { Underwater: } \\
\text { a) Diving } \\
\text { b) Viewing } \\
\text { vehicle }\end{array}$ & $\begin{array}{l}\text { Continuous viewing at 'animal } \\
\text { eye view', i.e. study spatial } \\
\text { relations and behavior from } \\
\text { an individual animal's actual } \\
\text { perspective. Continuous } \\
\text { detailed observations of } \\
\text { behavior and behavior } \\
\text { sequences. } \\
\text { Most detailed observations } \\
\text { possible }\end{array}$ & $\begin{array}{l}\text { Limited applicable habitats } \\
\text { Inability to study relatively } \\
\text { large scale spatial relations } \\
\text { Relatively high observer- } \\
\text { induced disturbance } \\
\text { potential } \\
\text { a) Short, animal-controlled } \\
\text { observation periods } \\
\text { b) Highly weather (sea state) } \\
\text { dependent } \\
\end{array}$ & $\begin{array}{l}\text { (Dudzinski et al., } \\
\text { 1995; Norris et } \\
\text { al., 1994; Ostman, } \\
\text { 1994; Rossbach \& } \\
\text { Herzing, 1997) }\end{array}$ \\
\hline $\begin{array}{l}\text { Boat-based: } \\
\text { a) Clear water } \\
\text { b) Opaque } \\
\text { water }\end{array}$ & $\begin{array}{l}\text { Ability to maintain contact } \\
\text { with focal-individual } \\
\text { a) Continuous viewing of } \\
\text { most behavior occurring } \\
\text { below water's surface }\end{array}$ & $\begin{array}{l}\text { Inability to study relatively } \\
\text { large scale spatial relations } \\
\text { Potential disturbance due to } \\
\text { boat presence } \\
\text { b) Observations often limited } \\
\text { to surface behaviors }\end{array}$ & $\begin{array}{c}\text { (Connor \& } \\
\text { Smolker, 1996; } \\
\text { Connor, Smolker } \\
\text { \& Richards, 1992; } \\
\text { Janik \& } \\
\text { Thompson, 1996; } \\
\text { Osborne, 1986; } \\
\text { Waples et al., } \\
\text { 1995) } \\
\end{array}$ \\
\hline Cliff & $\begin{array}{l}\text { Behavior not influenced by } \\
\text { observer } \\
\text { Ability to study large-scale } \\
\text { spatial relations/organization }\end{array}$ & $\begin{array}{l}\text { Inability to track individuals } \\
\text { of small species or large } \\
\text { groups } \\
\text { Limited underwater viewing } \\
\text { Limited behavioral detail } \\
\text { available } \\
\end{array}$ & $\begin{array}{c}\text { (Hanson \& } \\
\text { Defran, 1993; } \\
\text { Jefferson, 1987; } \\
\text { Norris } \text { et al., } \\
\text { 1994; Osborne, } \\
\text { 1986) } \\
\end{array}$ \\
\hline Theodolite & $\begin{array}{l}\text { Behavior not influenced by } \\
\text { observer } \\
\text { Track position/ movement of } \\
\text { identifiable individuals } \\
\text { Assess large scale movements, } \\
\text { e.g. responses to playback } \\
\text { experiments } \\
\text { Measure swim speed, distance } \\
\text { traveled }\end{array}$ & $\begin{array}{l}\text { Inability to track individuals } \\
\text { of small species or large } \\
\text { groups }\end{array}$ & $\begin{array}{l}\text { (Jefferson, 1987; } \\
\text { Malme et al., } \\
\text { 1983; Norris et } \\
\text { al., 1994; Sumich, } \\
\text { 1983) }\end{array}$ \\
\hline Airplane & $\begin{array}{l}\text { Disturbance by observer can } \\
\text { be minimized } \\
\text { Overhead viewing angle } \\
\text { minimizes reflection and } \\
\text { refraction } \\
\text { Mobile platform permits study } \\
\text { of many populations } \\
\text { Some sub-surface behavior } \\
\text { visible } \\
\text { Identifiable animals can be } \\
\text { followed for long periods }\end{array}$ & $\begin{array}{l}\text { Observed-induced } \\
\text { disturbance potentially } \\
\text { significant depending on } \\
\text { altitude } \\
\text { Disturbance mitigation } \\
\text { protocols reduce overhead } \\
\text { viewing advantage } \\
\text { Cannot follow individuals of } \\
\text { many species, nor see } \\
\text { behavioral detail }\end{array}$ & $\begin{array}{c}\text { (Hain et al., 1992; } \\
\text { Hubbs \& Hubbs, } \\
\text { 1967; Lowry et } \\
\text { al., 1996; Nerini } \\
\text { \& Rugh, 1986; } \\
\text { Watkins \& } \\
\text { Schevill, 1979; } \\
\text { Würsig et al., } \\
\text { 1984) }\end{array}$ \\
\hline
\end{tabular}

Table A1. Summary of behavioral observation techniques: advantages, disadvantages, applications and references (note: references are not meant to be exhaustive, simply representative). For 'Underwater' and 'Boat-based' categories, (a) and (b) distinctions apply to the entire row. 


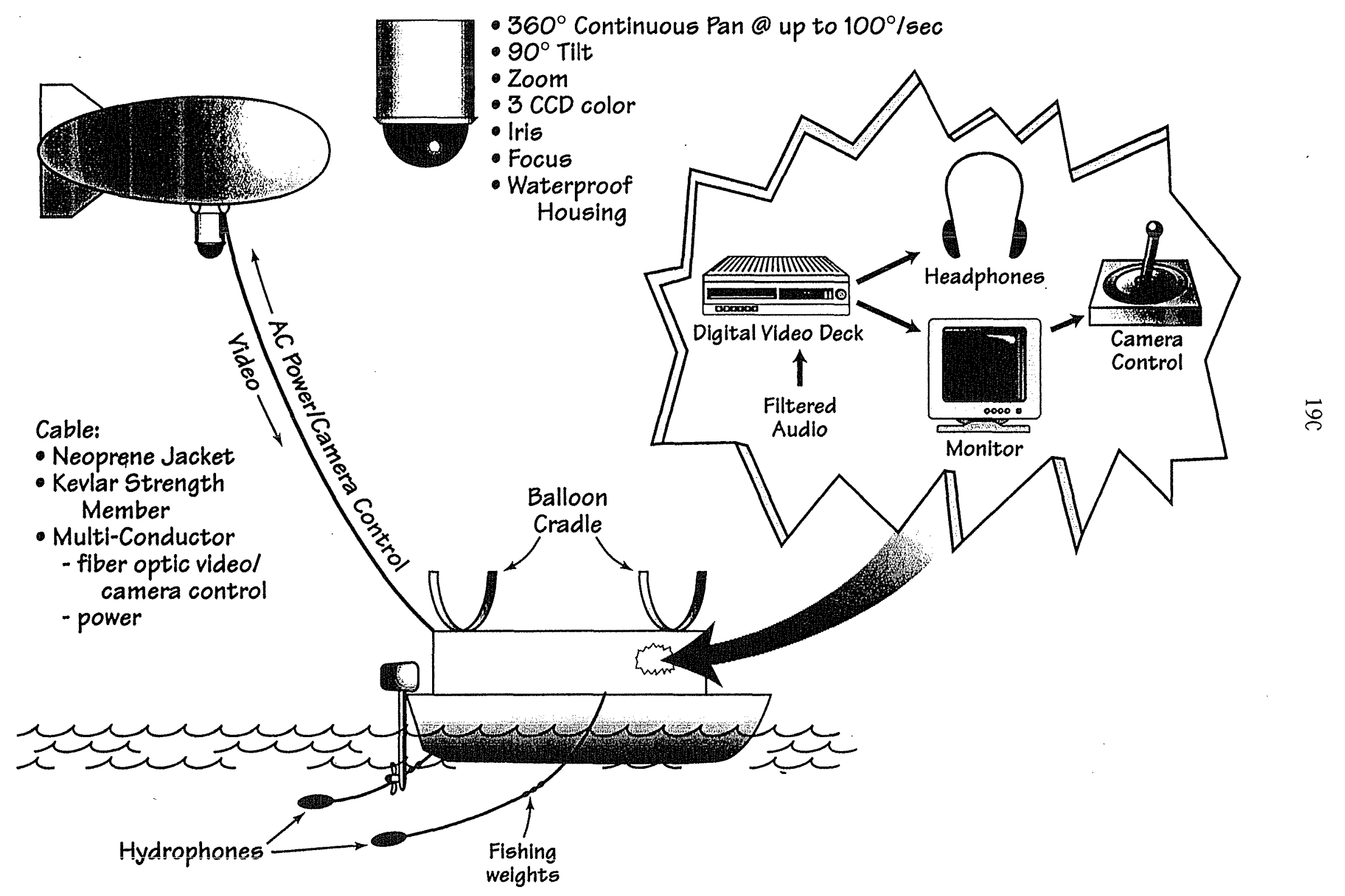

Figure 1. Schematic drawing of overhead video-ambient acoustic observation system. 


\section{A1.3 Literature Cited}

Altmann, J. (1974). Observational study of behavior: sampling methods. Behaviour 49, 227-267.

Connor, R. C. \& Smolker, R. A. (1996). 'Pop' goes the dolphin: a vocalization male botttlenose dolphins produce during courtships. Behaviour 133, 643-662.

Connor, R. C., Smolker, R. A. \& Richards, A. F. (1992). Two levels of alliance formation among male bottlenose dolphins (Tursiops sp.). Proceedings of the National Academy of Sciences USA 89, 987-990.

Dudzinski, K. M., Clark, C. W. \& Würsig, B. (1995). A mobile video/acoustic system for simultaneous underwater recording of dolphin interactions. Aquatic Mammals 21, 187-193.

Flamm, R. O., Owen, E. C. G., Weiss, C. F., Wells, R. S. \& Nowacek, D. P. (1999. In Prep). Aerial videogrammetry from a tethered airship to assess manatee life-stage structure. To be submitted to Marine Mammal Science.

Hain, J. H. W., Ratnaswamy, M. J., Kenney, R. D. \& Winn, H. E. (1992). The fin whale, Balaenoptera physalus, in waters of the northeastern United States continental shelf. Report of the International Whaling Commission 42, 653-669.

Hanson, M. T. \& Defran, R. H. (1993). The behaviour and feeding ecology of the Pacific coast bottlenose dolphin, Tursiops truncatus. Aquatic Mammals 19, 127-142.

Herzing, D. L. (1996). Vocalizations and associated underwater behavior of free-ranging Atlantic spotted dolphins, Stenella frontalis and bottlenose dolphins, Tursiops truncatus. Aquatic Mammals 22, 61-79.

Hubbs, C. L. \& Hubbs, L. C. (1967). Gray whale census by airplane in Mexico. California Fish and Game 53, 23-27.

Janik, V. M. \& Thompson, P. M. (1996). Changes in surfacing patterns of bottlenose dolphins in response to boat traffic. Marine Mammal Science 12, 597-602.

Jefferson, T. A. (1987). A study of the behavior of Dall's porpoise (Phocoenoides dalli) in the Johnstone Strait, British Columbia. Canadian Journal of Zoology 65, 736744.

Lowry, M. S., Perryman, W. L., Lynn, M. S., Westlake, R. L. \& Julian, F. (1996). Counts of northern elephant seals, Mirounga angustirostris, from large-format aerial photographs taken at rookeries in southern California during the breeding season. Fishery Bulletin 94, 176-185. 
Malme, C. I., Miles, P. R., Clark, C. W., Tyack, P. L. \& Bird, J. E. (1983). Investigations of the potential effects of underwater noise from petroleum industry activities on migrating gray whale behavior. U.S. Department of the Interior, Mineral Management Service.

Mann, J. (1999). Behavioral sampling methods for cetaceans: a review and critique. Marine Mammal Science 15, 102-122.

Miller, P. J. \& Tyack, P. L. (1998). A small towed beamforming array to identify vocalizing resident killer whales (Orcinus orca) concurrent with focal behavioral observations. Deep-Sea Research II 45, 1389-1405.

Nerini, M. K. \& Rugh, D. J. (1986). Distribution of bowhead whales in the vicinity of Point Barrow, Alaska: Aerial observations 1984 and 1985. Report of the International Whaling Commission 36, 359-361.

Norris, K. S. (1991). Dolphin days: the life and times of the spinner dolphin. Norton, New York.

Norris, K. S., Würsig, B., Wells, R. S. \& Würsig, M. (1994). The Hawaiian Spinner Dolphin. University of California Press, Berkeley.

Nowacek, D. P., Lange, W. N., Wells, R. S. \& Tyack, P. L. (1995). A new method for studying cetacean behavior and acoustics: overhead video combined with underwater audio. In Eleventh biennial conference on the biology of marine mammals, Orlando, FL.

Nowacek, S. M. (1999). The effects of boat traffic on bottlenose dolphins, Tursiops truncatus, in Sarasota Bay, Florida. Master of Science thesis, University of California Santa Cruz.

Osborne, R. W. (1986). A behavioral budget of Puget Sound killer whales. In Behavioral Biology of Killer Whales, vol. 1. Zoo Biology Monographs (ed. B. C. Kirkevold and J. S. Lockard), pp. 211-249. Alan R. Liss, New York.

Ostman, J. (1994). Social organization and social behavior of Hawai'ian spinner dolphins (Stenella longirostris). Ph.D. thesis, University of California Santa Cruz.

Perryman, W. L. \& Lynn, M. S. (1994). Examination of stock and school structure of striped dolphin (Stenella coeruleoalba) in the eastern Pacific from aerial photogrammetry. Fishery Bulletin 92, 122-131. 
Pryor, K. \& Norris, K. S. (1991). Dolphin Societies, Discoveries and Puzzles. University of California Press, Berkeley.

Richardson, W. J., Finley, K. J., Miller, G. W., Davis, R. A. \& Koski, W. R. (1995). Feeding, social and migration behavior of bowhead whales, Balaena mysticetus, in Baffin Bay vs. the Beaufort Sea - regions with different amounts of human activity. Marine Mammal Science 11, 1-45.

Richardson, W. J., Fraker, M. A., Würsig, B. \& Wells, R. S. (1985). Behaviour of bowhead whales (Balaena mysticetus) summering in the Beaufort Sea: Reactions to industrial activities. Biological Conservation 32, 195-230.

Richardson, W. J. \& Malme, C. I. (1993). Man-made noise and behavioral responses. In The Bowhead Whale (ed. J. J. Burns, J. J. Montague and C. J. Cowles), pp. 631700. The Society for Marine Mammalogy, Lawrence, KS.

Rohr, J., Endricks, E. W., Quigley, L., Fish, F. E., Gilpatrick, J. W. \& Scardina-Ludwig, J. (1998). Observations of dolphin swimming speed and strouhal number, pp. 48. Space and Naval Warfare Systems Center, San Diego.

Rossbach, K. A. \& Herzing, D. L. (1997). Underwater observations of benthic-feeding bottlenose dolphins (Tursiops truncatus) near Grand Bahama Island, Bahamas. Marine Mammal Science 13, 498-504.

Scott, M. D., Perryman, W. L. \& Clark, W. G. (1985). The use of aerial photographs for estimating school sizes of cetaceans, vol. 18, pp. 37. Bulletin, Inter-American Tropical Tuna Commission.

Smolker, R. A., Mann, J. \& Smuts, B. B. (1993). Use of signature whistles during separations and reunions by wild bottlenose dolphin mothers and infants. Behavioral Ecology and Sociobiology 33, 393-402.

Spikes, C. H. \& Clark, C. W. (1996). Whales 95 -- revolutionizing marine mammal monitoring technology. Sea Technology 37, 49-56.

Sumich, J. L. (1983). Swimming velocities, breathing patterns, and estimated costs of locomotion in migrating gray whales, Eschrichtius robustus. Canadian Journal of Zoology 61, 647-652.

Waples, D. M., Wells, R. S., Costa, D. P. \& Worthy, G. A. J. (1995). Seasonal changes in activity and habitat use by bottlenose dolphins (Tursiops truncatus) in Sarasota Bay, FL. In Eleventh Biennial Conference on the Biology of Marine Mammals, Orlando, FL. 
Watkins, W. A. \& Schevill, W. E. (1979). Aerial observation of feeding behavior in four baleen whales: Eubalaena glacialis, Balaenoptera borealis, Megaptera novaengliae, and Balaenoptera physalus. Journal of Mammology 60, 155-163.

Williams, J. (1970). Optical Properties of the Sea. United States Naval Institute, Annapolis.

Würsig, B., Dorsey, E. M., Fraker, M. A., Payne, R. S. \& Richardson, W. J. (1985). Behavior of bowhead whales, Balaena mysticetus, summering in the Beaufort Sea: a description. Fishery Bulletin 83, 357-377.

Würsig, B., Dorsey, E. M., Fraker, M. A., Payne, R. S., Richardson, W. J. \& Wells, R. S. (1984). Behavior of bowhead whales, Balaena mysticetus, summering in the Beaufort Sea: surfacing, respiration, and dive characteristics. Canadian Journal of Zoology 62, 1910-1921.

Würsig, B., Dorsey, E. M., Richardson, W. J. \& Wells, R. S. (1989). Feeding, aerial and play behaviour of the bowhead whale, Balaena mysticetus, summering in the Beaufort Sea. Aquatic Mammals 15, 27-37. 


\title{
Appendix 2. Acoustic Data Logger
}

\author{
Reprinted with permission from the Journal of the Acoustical Society of America
}

An onboard acoustic data logger to record biosonar of free-ranging bottlenose dolphins.

\author{
Douglas P. Nowacek*, Peter L. Tyack*, Randall S. Wellst, and Mark P. Johnson $\ddagger$
}

*Department of Biology and fDeparment of Applied Ocean Physics and Engineering, Woods Hole Oceanographic Institution, Woods Hole, MA 02543; †Chicago Zoological Society, clo Mote Marine Laboratory, Sarasota, FL 34236

Abstract: The ecology of the odontocete ccholocation system is not well understood despite a solid understanding of the system's operation. To gain insight into the functional uses of dolphin biosonar we have developed an acoustic data logger which utilizes a miniature DAT recorder and two suction-cup hydrophones. The first hydrophone is located $10 \mathrm{~cm}$ posterior of the blowhole. and the second $20 \mathrm{~cm}$ below the lateral base of the dorsal fin. The anterior 'high-frequency' hydrophone, designed specifically to record echolocation signals, has unity gain and a one-pole $10 \mathrm{kHz}$ high pass filter. The "ambient" hydrophone located at the base of the dorsal fin has $+18 \mathrm{~dB}$ gain and has a one-pole $1 \mathrm{kHz}$ high pass filter. To obtain echolocation recordings the "high-frequency" hydrophone was filtered through a simple demodulator in one of the deployments. The package was attached to temporarily restrained animals which, after release, were followed to recond behavioral data. During the two successful deployments to date the logger recorded animal vocalizations, surfacing events, the sounds of passing boats, and hydrodynamic sounds produced by the animal's fluke strokes.

\section{INTRODUCTION}

Odontocete cetaceans have been known for 37 years to use echolocation (Norris et al. 1961). The characteristies of the sonar system of the bottienose dolphin. Tursiops sruncalus, have been elucidated through intensive study of captive animals. Au (1993) reviews this research describing the dolphin transmission and receiving systems and documenting the characteristic acoustic features of the echolocation signals. We know also from these studies that the dolphins" sonar system is excellent for target detection, discrimination, and classification and for range discrimination (Au 1993). While the performance of the dolphin echolocation system is well characterized, its functional uses by wild animals are not well understood. Recent studies have begun to elucidate some details of odontocete echolocation use in the context of foraging (Verfuss and Schnitzler 1995; Miller et al. 1995). These studies document changes in echolocation signals and use patterns as the animals move through a predation sequence, a phenomenon also seen in foraging microchiropteran bats (Schnitzler \& Yenson 1980; Kick \& Simmons 1984).

Bat research has successfully elucidated many of the operational and functional details of echolocation. In addition to a good understanding of the performance (Schnitzler \& Henson 1980) and neural processing (Dear \& Suga 1995), the ecology of the bat echolocation system is much more fully understood than is the odontocete system (Neuweiler 1983; Surlykke 1988). In fact, few data exjst which can address even basic questions: how do odontocetes use echolocation for navigation and/or foraging? Do patterns of use change diumally?

\section{DATA LOGGER DESCRIPTION AND RESULTS}

One reason that odontucete echolocation research has not progressed as quickly as bat research is the difficulty in obtaining individually identified recordings of animals echolocating on biologically relevant targets. To procure such recordings we have developed an onboard acoustic data logger utilizing a two-channel DAT recorder housed in aluminum and attached to the dorsal fin with a Track Pack • (Figure 1). The recorder has a flat frequency response from $10 \mathrm{~Hz}-14 \mathrm{kHz}$, and each tape can store 120 stereo-minutes. The first suction-cup hydrophone (sensitivity $-205 \mathrm{~dB}$ re I $\mu \mathrm{Pa}$ ) is located $10 \mathrm{~cm}$ posterior of the blowhole, and the second $20 \mathrm{~cm}$ below the lateral base of the dorsal fin (Figure 1). The anterior "high-frequency" hydrophone, designed specifically to record echolocation signals, has unity gain and a one-pole $10 \mathrm{kHz}$ high pass filter. The 'ambient' hydrophone has $+18 \mathrm{~dB}$ gain and has a one-pole $1 \mathrm{kHz}$ high pass filter. To obtain echolocation recordings the 'high-frequency' hydrophone was filtered through a simple demodulator in one of the deployments. This frequency shift circuit is similar to a single-side-band demodulator, consisting of a high-pass filter (HPF) with passband edge at $70 \mathrm{kHz}$ and a multiplier (implemented by an analog switch), modulating the filtered signal with a $70 \mathrm{kHz}$ square wave. The result is that the $70-85 \mathrm{kHz}$ band is shifted 10 the $0-15 \mathrm{kHz}$ band of the DAT recorder. The purpose of the HPF is to minimize distortion in the recorded signal due to aliasing of the $55-70 \mathrm{kHz}$ band by the multiplier and the quality of the recording depends upon the stopband attenuation of the HPF. As the primary application of the frequency-shifted recordings was to be in estimating click rates, a high degree of alias rejection was not required. Given this and the small volume available in the tag for circuitry, we found that a straightforward combination of tunable notch and 3-pole active high-pass filters was satisfactory. VFF radio transmitters were mounted 
in the track pack for tracking the animal and recovering the package after it released via corrosible magnesium links. As part of the capture-release project in Sarasota, FL the package was attached to temporarily restrained animals which, after release, were followed to record behavioral data.

During the two successful deployments to date the logger recorded echolocation clicks (Figure 2), whistles (Figure 3), surfacing events, the sounds of passing boats, and hydrodynamic sounds produced by the animals' lluke strokes (Figure 2). The predominant activity observed for both subjects was traveling: no foraging or social behaviors were observed. One subject, a mature female, swam with her two-year old calf throughout the experiment, and both mother and calf surfaced with another dolphin one time. She may have exchanged sounds with her calf or another animal as whistles of alternating intensity were recorded. The second subject, a 9 year old female, did not behaviorally interact with any other animals, but she was well within acoustic range $(<100 \mathrm{~m})$ of other animals during the follow.

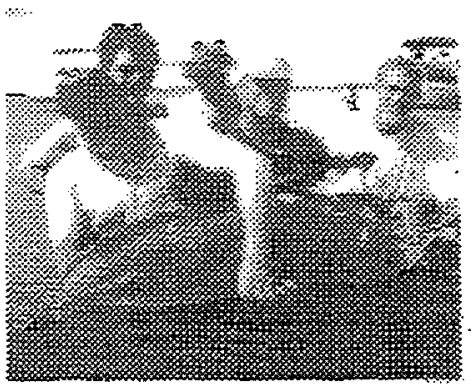

FIGURE 1. Data logger attached with a Track Pack Arrows show the positions of the hydrophones. The ambient sensor remained attached throughout all deployments, but the high frequency sensor released shortly before package release.

A slighty modified package is scheduled for further deployments in June, 1998. The modifications include a carbon fiber housing. a clock circuit to control the sampling interval, and pitch-roll-heading and depth sensors.

\section{ACKNOWLEDGMENTS}

Thanks to the Dolphin Biology Research Institute, Mote Marine Laboratory, Jim Cooper, Patrick Miller, Keith von der Heydt, Forest Townsend, and Frank Deckert. This work was supported WHOI's Coastal Research Center and Ocean Ventures Fund, and by ONR cuntract \#N00014-94-1-0692. This is contribution number 9669 from the Woods Hole Occanographic Institution.

\section{REFERENCES}

1. Au, W.W., The sonar of dolphins, New York: Springer-Verlag, 1993. 2. Dear, S.P., and Suga, N., Joumal of Neurophysiology, 73, 1084-1100 (1995).

3. Kick, S.A., and Simmons, J.A., The Joumal of Neuroscience, 4, 2725-2737 (1984).

4. Miller. LA., Pristed, J., Mohl, B, and Surlykke, A., Marine Mtammal Science, 11, 491-502 (1995).

5. Neuweiler, G., neuroethology and Behavioral Physiology, Berlin Springer-Verlag, 1983, pp. 280-302.

6. Norris, K.S., Prescott, J.H., Asa-Dorian, P.V., Perkins, P., Biological Bulletin, 120, 163 (1961).

7. Schnitler, H.-U., Henson, O.W.J., Animal Sonar Systems, New York: Plenum Press, 1980, pp. 109-181.

8. Surlykke, A. Animal Sonar: Processes and Performance. New York: Plenum Press, 1988, pp. 551-566.

9. Verfuss, U.K., Schnizzlet, H.U. "Hunting and echolocation behavior of a captive amazon river dolphin (Inia geoffensis), Eleventh biennial conference $n$ the biology of marine mammals, December 14-18,

Orlando, FL 1995.

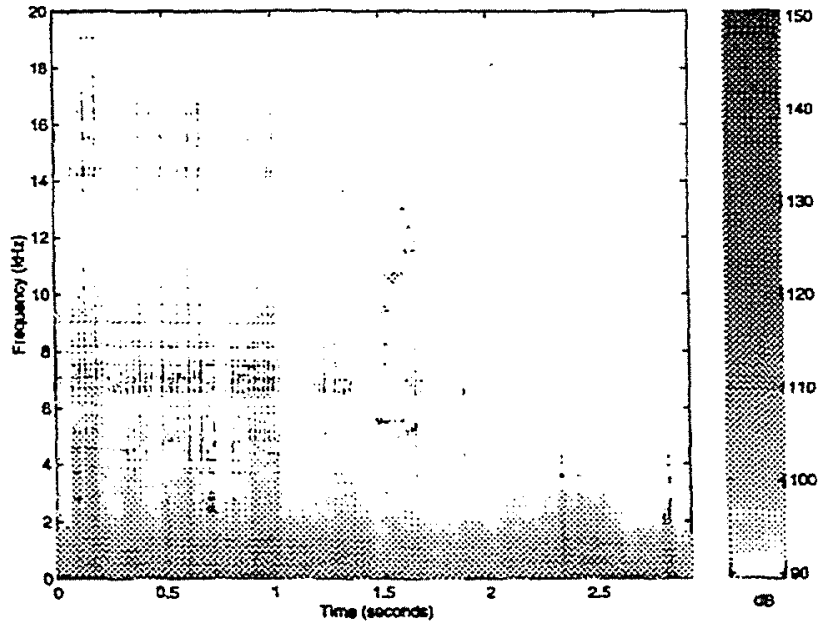

FIGURE 2. Echolocation clicks recorded by the 'ambient' hydrophone. The best clicks were recorded during the non-demodulator deployment. The received sound levels were calculated using a system component calibration (figures 2 \&). The flukc strokes appear as oscillations in the 'noise floor' $(<4 \mathrm{kHz})$.

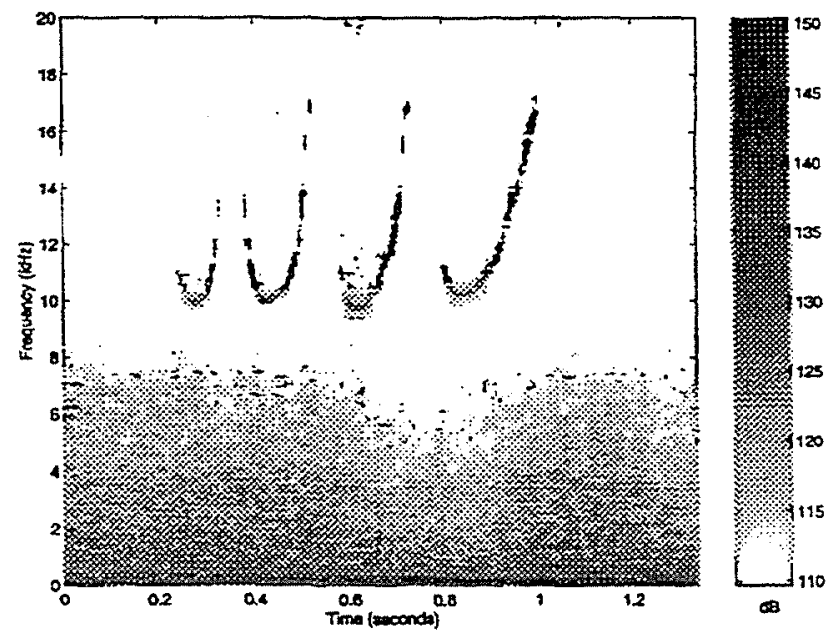

FIGURE 3. Whistles recorded by the 'ambient' hydrophane. Due to the directionality and frequency of clicks as compared to whistles it is not surprising that the whistles produced a greater received level. Nole the difference in received level scale. 